Wagner Shin Nishitani

\title{
Projeto de Micromecanismos Multifásicos Usando o Método da Otimização Topológica
}

\author{
Dissertação apresentada à Escola \\ Politécnica da Universidade de São \\ Paulo para obtenção do Título de \\ Mestre em Engenharia Mecânica.
}


Wagner Shin Nishitani

\section{Projeto de Micromecanismos Multifásicos Usando o Método da Otimização Topológica}

Dissertação apresentada à Escola Politécnica da Universidade de São Paulo para obtenção do Título de Mestre em Engenharia Mecânica.

Área de concentração:

Engenharia de Controle e Automação Mecânica

Orientador:

Prof. Dr. Emílio Carlos Nelli Silva 
A meus pais e minha irmã 


\section{Agradecimentos}

A Deus, a quem devo muito por todas as coisas em minha vida.

À minha família, por tudo o que foi imprescindível no meu crescimento: exemplo, zêlo, respeito e amor.

Ao professor Emilio, não só pela orientação sempre próxima e cuidadosa, mas também pelo compartilhamento de experiências e encorajamento nos momentos difíceis.

Ao meus colegas de pós-graduação, em especial ao Wilfredo, Stump e Ronny, pela ajuda durante o desenvolvimento do presente trabalho. Agradeço não só a estes, mas a tantos outros pelo apoio moral e momentos de descontração que foram muito importantes.

A Fundação de Amparo à Pesquisa do Estado de São Paulo (FAPESP), pela concessão da bolsa de estudos para o período de realização dos trabalhos de mestrado.

Ao Laboratório Nacional de Luz Síncrotron (LNLS), pela utilização do Laboratório de Microfabricação (LMF) na investigação realizada para a fabricação de micromecanismos multifásicos. Em especial, agradeço à Maria Helena e ao Angelo pelo apoio e incentivo. 


\section{Resumo}

Um micromecanismo é, essencialmente, um dispositivo de dimensões milimétricas ou até micrométricas que executa uma tarefa específica como atuar como garra, pinça, grampo, etc. Quando acoplados a um sistema eletrônico, são chamados de sistemas microeletromecânicos ou "Micro-Electro-Mechanical Systems" (MEMS). Esses dispositivos são quase todos constituídos por mecanismos flexíveis, onde o movimento é dado pela flexibilidade de sua estrutura, sem juntas e pinos. Uma das formas de atuação de micromecanismos é a eletrotermomecânica, onde uma atuação elétrica sobre o próprio mecanismo é convertida em calor, por efeito Joule, que gera tensões térmicas responsáveis pela deformação estrutural desejada. Recentemente, vários grupos de pesquisa no mundo estão desenvolvendo micromecanismos fabricados com dois (ou até mais) materiais, o que permite obter maiores deformações sem que seja excedido o limite de resistência do material e mais flexibilidade no projeto de micromecanismos que realizem diferentes tarefas quando sujeito a diversas atuações (multiflexíveis).

As técnicas de processo de fabricação de micromecanismos atingiram um alto nível de maturidade. No entanto, a modelagem e, em particular, o desenvolvimento de métodos computacionais sistemáticos para o projeto estão ainda no seu estágio inicial. Atualmente, o projeto de micromecanismos com vários materiais vem sendo realizado por métodos de tentativa e erro, dependendo da intuição e experiência do projetista. Além disso, o projeto genérico de um MEMS eletrotermomecânico é uma tarefa complexa, que leva em conta conhecimentos multidisciplinares.

Dessa forma, o objetivo desse trabalho de mestrado foi desenvolver um software para o projeto de MEMS multifásicos, atuados eletrotermicamente, usando um método de projeto genérico e sistemático, como o Método de Otimização Topológica (MOT). Utilizando um modelo de interpolação de material de função de pico, qualquer número de materiais pode ser considerado sem que haja aumento na quantidade de variáveis de projeto se comparado à otimização com apenas um material e vazio. Visando maximizar o deslocamento de saída contra uma peça de rigidez conhecida, foram projetados mecanismos atuados por tensão elétrica, alguns considerando multiflexibilidade. Um estudo da influência dos parâmetros da otimização foi realizado. Como uma alternativa à atuação eletrotermomecânica, foram projetados mecanismos atuados por fluxo de calor. 


\section{Abstract}

A micromechanism is essentially a device of milimetric, or even micrometric, dimensions that can actuate as a gripper, tweezers, clamp, etc. When coupled to an electronic system, they are called "Micro-Electro-Mechanical Systems" (MEMS). Almost all of these devices are constituted by compliant mechanisms, where the motion is allowed by the compliance of its own structure, rather than the presence of joint and pins. One of the forms of micromechanisms actuation is the electrothermomechanical, where an electric actuation applied to the mechanism is converted in heat, by Joule effect, that generates the thermal stress responsible for the desired structural deformation. Recently, many research groups around the world are developing micromechanisms manufactured with two (or even more) materials, what allows larger displacements without exceeding the materials ultimate tensile strength, and gives more flexibility in the design of micromechanisms that accomplish different tasks when under different actuations (multiflexible mechanisms).

The manufacturing process techniques of micromechanisms reached a high level of maturity, however, the modelling and, particularly, the development of systematic computational methods for design are still in early stages. Nowadays, micromechanism design with many materials are being carried on by "try and error" methods, depending on designer intuition and experience. Also, a generic design of an electrothermomechanical MEMS is a complex task that needs multidisciplinary knowledge.

Thus, the objective of this work is to develop a software for the design of multi-phase MEMS, electrothermomechanically actuated, using a method for systematic and generic design, such as Topology Optimization Method (TOM). Using a peak function material interpolation model, any number of materials can be considered without increasing the amount of design variables if compared to an optimization with only one material and void. Mechanisms actuated by electric tension were designed considering the maximization of output displacement against a work piece with known stiffness. The design of microactuators considering multiflexibility was also performed. A study of optimization parameters influence is presented. As an alternative to electrothermomechanical actuation, some mechanisms actuated by heat flow were designed. 


\title{
Conteúdo
}

\section{Lista de Figuras}

\author{
Lista de Tabelas
}

\section{Lista de Abreviaturas}

\section{Convenções e Lista de Símbolos}

1 Introdução 1

1.1 MEMS Eletrotermomecânicos . . . . . . . . . . . . 1

1.2 O problema do projeto de MEMS eletrotermomecânicos . . . . . 5

1.3 Método de Otimização Topológica aplicado ao projeto de MEMS eletrotermomecânicos . . . . . . . . . . . 7

1.4 Objetivo e Justificativa . . . . . . . . . . . . . 9

2 Modelagem de MEMS Eletrotermomecânicos pelo MEF 10

2.1 Equações governantes dos micromecanismos eletrotermomecânicos 10

2.2 Formulação do Método de Elementos Finitos . . . . . . . . . . 13

2.2.1 MEF no problema termoelástico . . . . . . . . . 15

2.2.2 MEF no problema elétrico e eletrotérmico . . . . . . . 23

3 O Método de Otimização Topológica (MOT) 27

3.1 Introdução . . . . . . . . . . . . . . . . 27

3.2 Conceitos Básicos . . . . . . . . . . . . . . . 29

3.2.1 Domínio Fixo Estendido de Projeto . . . . . . . . . . 30

3.2.2 Modelo de Interpolação de Material . . . . . . . . . . . 31 
3.3 Desenvolvimento do MOT . . . . . . . . . . . 35

3.3.1 Histórico ........................ 35

3.3.2 Utilização de múltiplos materiais . . . . . . . . . . 37

3.4 Problemas Numéricos . . . . . . . . . . . . . . . 39

3.4.1 Dependência de malha ............. 40

3.4 .2 Não unicidade da solução . . . . . . . . . . . 40

3.4.3 Escalas de cinza (ou "grayscale") ............ 41

3.4.4 Instabilidade de tabuleiro (ou "checkerboard") . . . . . . 42

4 Formulação do Problema de Otimização de MEMS Eletrotermomecânicos Multifásicos $\quad 46$

4.1 Formulação do Problema . . . . . . . . . . . . . . . 46

4.2 Modelos de Interpolação para Múltiplos Materiais . . . . . . . . . 49

4.2.1 Extensão do "Simple Isotropic Material with Penalization" $(\mathrm{SIMP}) \ldots \ldots \ldots \ldots . \ldots \ldots$

4.2.2 Função de Pico . . . . . . . . . . . . . . 50

4.3 Análise de sensibilidades . . . . . . . . . . . . . 52

4.3.1 Sensibilidade através do método adjunto . . . . . . 53

4.3.2 Sensibilidade - Matrizes de rigidez $\boldsymbol{K}_{\mathbf{2}}$, de condutividade térmica $\boldsymbol{K}_{\mathbf{1}}$ e de condutividade elétrica $\boldsymbol{K}_{\mathbf{0}} \ldots \ldots \ldots$. . 58

4.3.3 Sensibilidade - Vetor de carga estrutural $\boldsymbol{P}_{\mathbf{2}} \ldots \ldots \ldots$

4.3.4 Sensibilidade - Vetor de carga térmica $\boldsymbol{P}_{\mathbf{1}} \ldots \ldots$. . . 60

4.3.5 Sensibilidade - Vetor de carga elétrica $\boldsymbol{P}_{\mathbf{0}} \ldots \ldots 62$

4.3.6 Sensibilidade - Funções de restrição de volume $\boldsymbol{G}_{\boldsymbol{v o l}_{\boldsymbol{m}}}$. . . 62

4.3.7 Sensibilidade - Propriedades dos materiais . . . . . . . 63

5 Implementação Numérica do MOT $\quad 64$

5.1 Técnicas de solução do problema de otimização . . . . . . . . . 64

5.1.1 Programação Linear Sequencial (PLS) . . . . . . . . 65

5.2 Implementação do MOT . . . . . . . . . . . . . . . 71 
5.3 Filtro Implementado . . . . . . . . . . . . . . . 75

$\begin{array}{llr}6 & \text { Resultados } & 77\end{array}$

6.1 Atuador de movimento inverso . . . . . . . . . . 77

6.1.1 Uso de dois materiais, além do vazio . . . . . . . 78

6.1.2 Uso de três materiais, além do vazio . . . . . . . . 85

6.2 Influência dos Parâmetros da Otimização . . . . . . . . . . . 89

6.2.1 Rigidez da Peça Atuada . . . . . . . . . . . . 89

6.2.2 Restrições de Volume por Material . . . . . . . . . . 90

6.2.3 Uso do Filtro Espacial . . . . . . . . . . . . . 92

6.2.4 Coeficiente de Continuação dos Desvios Padrão . . . . . 97

6.2.5 Parâmetros da Função de Pico - Média e Desvio Padrão 100

6.3 Atuador multiflexível . . . . . . . . . . . . . . . . . 101

6.3.1 Uso de dois materiais, além do vazio . . . . . . . . . 103

6.3.2 Uso de um material, além do vazio . . . . . . . . . . . 112

6.4 Atuador de movimento inverso atuado por calor . . . . . . . . 122

6.4.1 Uso de dois materiais, além do vazio . . . . . . . . . 123

6.4.2 Uso de três materiais, além do vazio . . . . . . . . . . 128

7 Conclusões e Sugestão de Trabalhos Futuros 134

$\begin{array}{ll}\text { Referências } & 137\end{array}$

Apêndice A - Imposição de condições de contorno de Dirichlet 147

Apêndice B - Verificações da Implementação 149

B.1 Verificação do Método de Elementos Finitos (MEF) . . . . . . . 149

B.2 Verificação das Sensibilidades . . . . . . . . . . . . 161

Apêndice C - Atividades Realizadas no Laboratório Nacional de $\begin{array}{lr}\text { Luz Síncrotron (LNLS) } & 163\end{array}$ 


\section{Lista de Figuras}

1.1 Exemplo de um MEMS eletrotermomecânico do tipo "V" aberto e sua forma sob atuação. . . . . . . . . . . . . . . . 3

1.2 Exemplo de um MEMS eletrotermomecânico pseudo-bilaminar e sua forma sob atuação. . . . . . . . . . . . . . . . 4

1.3 Exemplo de um MEMS eletrotermomecânico pseudo-bilaminar uniaxial e sua forma sob atuação. . . . . . . . . . . . . 4

1.4 Exemplo de um micromecanismo multifásico e multi-atuado. . . . 5

1.5 Projeto de um micromecanismo multifásico multiflexível. . . . . . 6

2.1 Condições de carga e fronteira para o problema eletrotermomecânico. 11

2.2 Elemento retangular de quatro nós. . . . . . . . . . . . . 16

2.3 Tensões normais e de cisalhamento em $x$ e $y \ldots \ldots 22$

3.1 Otimização Paramétrica de uma viga em balanço. . . . . . . . . 28

3.2 Otimização de Forma de uma viga em balanço. . . . . . . . . 28

3.3 Otimização Topológica de uma viga em balanço. . . . . . . . . . . 29

3.4 Exemplo de Elementos no Método de Homogeneização. . . . . . . 32

3.5 Mínimos dos problemas convexo e não-convexo. . . . . . . . . . . 41

3.6 Exemplo de aparecimento de escalas de cinza. . . . . . . . . . . 42

3.7 Exemplo de aparecimento de instabilidade de tabuleiro. . . . . . 43

4.1 Problema do projeto de um micromecanismo eletrotermomecânico. 47

4.2 Problema do projeto de um micromecanismo eletrotermomecânico multiflexível. . . . . . . . . . . . . . . . . . . . . 48

4.3 Exemplo de propriedade interpolada pela função de pico. . . . . . 51

5.1 Programação Linear Sequencial e os limites móveis. . . . . . . . 66

5.2 Fluxograma do Método de Otimização Topológica. . . . . . . . 72 
5.3 Diagrama de fluxo para os subproblemas de MEF. . . . . . . 73

5.4 Raio de ação do filtro espacial. . . . . . . . . . . . . . 75

6.1 Domínio fixo estendido para o atuador de movimento inverso. . . 78

6.2 Interpolação dos Módulos de Young de $\mathrm{Si}$ e $\mathrm{SiO}_{2}$. . . . . . . . 79

6.3 Atuador de movimento inverso - Topologias intermediárias. . . . . 80

6.4 Atuador de movimento inverso - Função Objetivo. . . . . . . . . . 80

6.5 Atuador de movimento inverso - Topologia final e Análises. . . . . 81

6.6 Atuador de movimento inverso (com restrições de volume) Topologias intermediárias. . . . . . . . . . . . 83

6.7 Atuador de movimento inverso (com restrições de volume) Função Objetivo. . . . . . . . . . . . . . . 83

6.8 Atuador de movimento inverso (com restrições de volume) Topologia final e Análises. . . . . . . . . . . . . . . . 84

6.9 Interpolação dos Módulos de Young de $\mathrm{Si}, \mathrm{SiO}_{2}$ e Ni. . . . . . . 86

6.10 Atuador de movimento inverso (3 materiais) - Topologias intermediárias. .................. 86

6.11 Atuador de movimento inverso (3 materiais) - Função Objetivo. . 87

6.12 Atuador de movimento inverso (3 materiais) - Topologia final e Análises. . . . . . . . . . . . . . . . . . 88

6.13 Volume Relativo dos resultados para o atuador de movimento inverso. 89

6.14 Volumes Relativos dos resultados para o atuador de movimento inverso. . . . . . . . . . . . . . . . . . . 91

6.15 Atuador de movimento inverso - Comparação de topologias (restrições de volume variadas continuamente). . . . . . . . . . . . 91

6.16 Volumes Relativos para o atuador de movimento inverso (restrições de volume variadas continuamente) . . . . . . . . . . . . 92

6.17 Atuador de movimento inverso (com maior discretização) Topologias intermediárias. . . . . . . . . . . . . . 93

6.18 Atuador de movimento inverso (com maior discretização) Comparação de topologias (diferentes discretizações). . . . . . . . 93 
6.19 Atuador de movimento inverso (com filtro até iteração 100) Topologias intermediárias. . . . . . . . . . . . . . . . 94

6.20 Atuador de movimento inverso (com filtro até iteração 100) Função Objetivo. . . . . . . . . . . . . . . . 94

6.21 Atuador de movimento inverso (sem filtro) - Topologias intermediárias. .................... 95

6.22 Atuador de movimento inverso (sem filtro) - Função Objetivo. . . 95

6.23 Atuador de movimento inverso (com restrições de volume, sem filtro) - Topologias intermediárias. . . . . . . . . . . 96

6.24 Atuador de movimento inverso (com restrições de volume, sem filtro) - Função Objetivo. . . . . . . . . . . . . . 96

6.25 Atuador de movimento inverso (com restrições de volume, $\omega_{D P_{m}}=$ $0,005(m=1$ e 2$))$ - Topologias intermediárias. . . . . . . 98

6.26 Atuador de movimento inverso (com restrições de volume, $\omega_{D P_{m}}=$ $0,005(m=1$ e 2$))$ - Função Objetivo. . . . . . . . . 98

6.27 Atuador de movimento inverso (com restrições de volume, $\omega_{D P_{m}}=$ $0,02(m=1$ e 2$))$ - Topologias intermediárias. . . . . . . 99

6.28 Atuador de movimento inverso (com restrições de volume, $\omega_{D P_{m}}=$ $0,02(m=1$ e 2$))$ - Função Objetivo. . . . . . . . . 99

6.29 Atuador de movimento inverso (com restrições de volume, função de pico modificada) - Topologias intermediárias. . . . . . . . . 100

6.30 Atuador de movimento inverso (com restrições de volume, função de pico modificada) - Função Objetivo. . . . . . . . . . . . . . 101

6.31 Domínio fixo estendido para o atuador multiflexível. . . . . . . . . 102

6.32 Atuador multiflexível - Topologias intermediárias. . . . . . . . . . 104

6.33 Atuador multiflexível - Curvas da otimização. . . . . . . . . . . 105

6.34 Atuador multiflexível - Topologia final e interpretada, Análise termoelástica. . . . . . . . . . . . . . 106

6.35 Atuador multiflexível - Análise elétrica e eletrotérmica. . . . . . . 107

6.36 Atuador multiflexível (com restrições de volume) - Topologias intermediárias. 
6.37 Atuador multiflexível (com restrições de volume) - Curvas da otimização. . . . . . . . . . . . . . . . . . 109

6.38 Atuador multiflexível (com restrições de volume) - Topologia final e interpretada, Análise termoelástica. . . . . . . . . . . . 110

6.39 Atuador multiflexível (com restrições de volume) - Análise elétrica e eletrotérmica. . . . . . . . . . . . . . . . . . . 111

6.40 Atuador multiflexível (em cobre) - Topologias intermediárias (vermelho - Ni; verde - Cu). . . . . . . . . . . 113

6.41 Atuador multiflexível (em cobre) - Curvas da otimização. . . . . . 114

6.42 Atuador multiflexível (em cobre) - Topologia final e interpretada, Análise termoelástica. . . . . . . . . . . . . 115

6.43 Atuador multiflexível (em cobre) - Análise elétrica e eletrotérmica. 116

6.44 Atuador multiflexível (em cobre, com restrições de volume) Topologias intermediárias. . . . . . . . . . . . . 118

6.45 Atuador multiflexível (em cobre, com restrições de volume) Curvas da otimização. . . . . . . . . . . . . . . . 119

6.46 Atuador multiflexível (em cobre, com restrições de volume) Topologia final e interpretada, Análise termoelástica. . . . . . . 120

6.47 Atuador multiflexível (em cobre, com restrições de volume) Análise elétrica e eletrotérmica. . . . . . . . . . . . . 121

6.48 Domínio fixo estendido para o atuador de movimento inverso atuado por calor. . . . . . . . . . . . . . . 122

6.49 Atuador de movimento inverso atuado por calor - Topologias intermediárias.

6.50 Atuador de movimento inverso atuado por calor - Curvas da otimização.

6.51 Atuador de movimento inverso atuado por calor - Topologia final e Análises.

6.52 Atuador de movimento inverso atuado por calor (com restrições de volume) - Topologias intermediárias. . . . . . . . . . . 126

6.53 Atuador de movimento inverso atuado por calor (com restrições de volume) - Curvas da otimização. 
6.54 Atuador de movimento inverso atuado por calor (com restrições de volume) - Topologia final e Análises. . . . . . . . . . . . 127

6.55 Atuador de movimento inverso atuado por calor (3 materiais) Topologias intermediárias. . . . . . . . . . . . . . . 129

6.56 Atuador de movimento inverso atuado por calor (3 materiais) Curvas da otimização. . . . . . . . . . . . . . . . 129

6.57 Atuador de movimento inverso atuado por calor (3 materiais) Topologia final e Análises. . . . . . . . . . . . . . . . 130

6.58 Atuador de movimento inverso atuado por calor (3 materiais, com restrições de volume) - Topologias intermediárias. . . . . . . . . . 131

6.59 Atuador de movimento inverso atuado por calor (3 materiais, com restrições de volume) - Curvas da otimização. . . . . . . . . . . 132

6.60 Atuador de movimento inverso atuado por calor (3 materiais, com restrições de volume) - Topologia final e Análises. . . . . . . . . . 132

B.1 Modelo para verificação do MEF. . . . . . . . . . . . . 150

B.2 Resultados das Análises por ANSYS (sem convecção). . . . . . . . 151

B.3 Resultados das Análises pelo programa em C (sem convecção). . . 152

B.4 Resultados das Análises por ANSYS (com convecção). . . . . . . 156

B.5 Resultados das Análises pelo programa em C (com convecção matriz $h_{A N S Y S}^{e} \ldots \ldots \ldots \ldots \ldots \ldots \ldots$

B.6 Resultados das Análises pelo programa em C (com convecção -

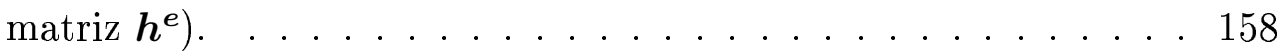

B.7 Modelo para verificação das sensibilidades. . . . . . . . . . . . 161

C.1 Etapas da Microfabricação de um Mecanismo Bimetálico. . . . . . 164

C.2 Microposicionador $x y$ Bimetálico - Esquema. . . . . . . . . . . . 164

C.3 Microposicionador $x y$ Bimetálico - Protótipo. . . . . . . . . . 165

C.4 Microposicionador $x y$ Bimetálico - Junções níquel-cobre. . . . . . 165 


\section{Lista de Tabelas}

6.1 Propriedades dos Materiais - Silício e Dióxio de silício. . . . . 78

6.2 Atuador de movimento inverso - Características da topologia final. 82

6.3 Atuador de movimento inverso (com restrições de volume) Características da topologia final. . . . . . . . . . . 82

6.4 Propriedades dos Materiais - Níquel. . . . . . . . . . 85

6.5 Atuador de movimento inverso (3 materiais) - Características da topologia final. . . . . . . . . . . . . . 87

6.6 Atuador de movimento inverso - Rigidez da peça atuada e deslocamentos de saída da topologia final. . . . . . . . . . 90

6.7 Propriedades dos Materiais - Níquel e Cobre. . . . . . . . . . 102

6.8 Atuador multiflexível - Características da topologia interpretada. 104

6.9 Atuador multiflexível (com restrições de volume) - Características da topologia interpretada. . . . . . . . . . . . . . 112

6.10 Atuador multiflexível (em cobre) - Características da topologia interpretada. . . . . . . . . . . . . . . . 113

6.11 Atuador multiflexível (em cobre, com restrições de volume) Características da topologia interpretada. . . . . . . . . . 118

6.12 Atuador de movimento inverso atuado por calor - Características da topologia final. . . . . . . . . . . . . . . . . 123

6.13 Atuador de movimento inverso atuado por calor (com restrições de volume) - Características da topologia final. . . . . . . . 126

6.14 Atuador de movimento inverso atuado por calor (3 materiais) Características da topologia final. . . . . . . . . . . 130

6.15 Atuador de movimento inverso atuado por calor (3 materiais, com restrições de volume) - Características da topologia final. . . . . . 133

B.1 Propriedades dos Materiais - Níquel e Cobre. . . . . . . . . . 149 
B.2 Resultados da Análise Elétrica - Tensões Elétrica. . . . . . . . . 150

B.3 Resultados da Análise Elétrica - Densidades de Corrente. . . . . . 153

B.4 Resultados da Análise Eletrotérmica (sem conveç̧ão) Temperaturas. . . . . . . . . . . . . . . . . . 153

B.5 Resultados da Análise Termoelástica (sem conveç̧ão) Deslocamentos. . . . . . . . . . . . . . . 154

B.6 Resultados da Análise Termoelástica (sem convecção) - Tensões Mecânicas (programa em C). . . . . . . . . . . 154

B.7 Resultados da Análise Termoelástica (sem convecção) - Tensões Mecânicas (ANSYS). . . . . . . . . . . . . 155

B.8 Resultados da Análise Eletrotérmica (com conveç̧ão) Temperaturas. . . . . . . . . . . . . . 155

B.9 Resultados da Análise Termoelástica (com conveç̧ão) Deslocamentos. . . . . . . . . . . . . . . 159

B.10 Resultados da Análise Termoelástica (com convecção) - Tensões Mecânicas $\left(\right.$ programa em C $\left.-\boldsymbol{h}^{e}\right) \ldots \ldots \ldots . . \ldots 159$

B.11 Resultados da Análise Termoelástica (com convecção) - Tensões Mecânicas (programa em C - $\boldsymbol{h}_{\boldsymbol{A N S Y} \text { S }}^{e}$. . . . . . . . 160

B.12 Resultados da Análise Termoelástica (com convecção) - Tensões Mecânicas (ANSYS). . . . . . . . . . . . 160

B.13 Cálculo das Sensibilidades pelo método adjunto e Método de Diferenças Finitas. . . . . . . . . . . . . . . 162 


\section{Lista de Abreviaturas}

CAD "Computer-Aided Design"

CAMD "Continuous Approximation of Material Distribution"

KS Kreisselmeier-Steinhauser

LMF Laboratório de Microfabricação

LNLS Laboratório Nacional de Luz Síncrotron

LSI Laboratório de Sistemas Integráveis

MDF Método de Diferenças Finitas

MEF Método de Elementos Finitos

MEMS "Micro-Electro-Mechanical Systems"

MMA "Method of Moving Asymptotes"

MOT Método de Otimização Topológica

OT Otimização Topológica

PL Programação Linear

PLS Programação Linear Sequencial

PQS Programação Quadrática Sequencial

SIMP "Simple Isotropic Material with Penalization" 


\section{Convenções e Lista de Símbolos}

Os seguintes símbolos serão utilizados:

\begin{tabular}{|c|c|}
\hline símbolo & descrição \\
\hline$\Omega$ & Domínio que contém o sólido sob análise \\
\hline$\Gamma$ & Superfície de fronteira do domínio $\Omega$ \\
\hline$\Gamma_{u_{i}}$ & $\begin{array}{l}\text { Fronteira com condições de deslocamento para o } \\
\text { domínio } i(i=0,1 \text { ou } 2 \text { - elétrico, térmico e elástico })\end{array}$ \\
\hline$\Gamma_{t_{i}}$ & $\begin{array}{l}\text { Fronteira com cargas superficiais para o domínio } i(i= \\
0,1 \text { ou } 2 \text { - elétrico, térmico e elástico })\end{array}$ \\
\hline$u_{0}$ & Tensão elétrica \\
\hline$u_{1}$ & $\begin{array}{l}\text { Variação de temperatura em relação ao estado livre de } \\
\text { tensões térmicas }\end{array}$ \\
\hline$u_{2(i)}$ & Componente do deslocamento numa direção $i$ \\
\hline,$i$ & Diferenciação com respeito à direção da coordenada $i$ \\
\hline$b_{0}$ & $\begin{array}{l}\text { Fonte prescrita de corrente interna por unidade de } \\
\text { volume }\end{array}$ \\
\hline$h$ & Coeficiente de convecção \\
\hline$b_{1}$ & $\begin{array}{l}\text { Geração interna de calor por unidade de volume e } \\
\text { convecção da superfície superior }\end{array}$ \\
\hline$b_{2(i)}$ & Componentes de força no corpo \\
\hline$\sigma_{0}$ & Condutividade elétrica (isotrópica) \\
\hline$\sigma_{1}$ & Condutividade térmica (isotrópica) \\
\hline$E_{i j k l}$ & Tensor de rigidez elástica \\
\hline $\bar{u}_{0}$ & Tensão elétrica prescrita \\
\hline $\bar{u}_{1}$ & Variação de temperatura - prescrita \\
\hline $\bar{u}_{1 \infty}$ & Variação de temperatura - ambiente \\
\hline $\bar{u}_{2(i)}$ & Componentes de deslocamentos prescritas \\
\hline $\bar{t}_{0}$ & Corrente elétrica prescrita \\
\hline $\bar{t}_{1}$ & Fluxo de corrente e calor prescrito \\
\hline $\bar{t}_{2(i)}$ & Componentes de força de tração \\
\hline$\eta_{i j}$ & Tensor de deformações de engenharia \\
\hline
\end{tabular}

continua... 


\begin{tabular}{|c|c|}
\hline símbolo & descrição \\
\hline$s_{i j}$ & Tensor de tensões mecânicas \\
\hline$\alpha_{i j}$ & Tensor de expansão térmica \\
\hline$n_{i}$ & $\begin{array}{l}\text { Componentes do vetor normal unitário para a superfície } \\
\Gamma\end{array}$ \\
\hline$N$ & Número de elementos usados na discretização \\
\hline$n$ & Número de nós da discretização \\
\hline$\rho^{e}$ & Pseudo-densidade do elemento $e$ \\
\hline$D$ & Matriz de elasticidade \\
\hline$\alpha$ & Coeficiente de expansão térmica (isotrópico) \\
\hline$\rho$ & Vetor das variáveis de projeto de todo o domínio \\
\hline$K_{0}$ & Matriz de condutividade elétrica global \\
\hline$K_{1}$ & Matriz de condutividade térmica global \\
\hline$K_{2}$ & Matriz de rigidez global \\
\hline$U_{0}$ & Vetor das tensões elétricas nodais \\
\hline$U_{1}$ & $\begin{array}{l}\text { Vetor das variações de temperatura nodais em relação } \\
\text { ao estado livre de tensões térmicas }\end{array}$ \\
\hline$U_{2}$ & Vetor dos deslocamentos nodais \\
\hline$P_{0}$ & Vetores das cargas elétricas nodais \\
\hline$P_{1}$ & Vetores das cargas térmicas nodais \\
\hline$P_{2}$ & Vetor das cargas elásticas nodais \\
\hline$K_{0}^{e}$ & Matriz de condutividade elétrica do elemento $e$ \\
\hline$K_{1}^{e}$ & Matriz de condutividade térmica do elemento $e$ \\
\hline$K_{2}^{e}$ & Matriz de rigidez do elemento $e$ \\
\hline$h^{e}$ & Matriz de convecção do elemento $e$ \\
\hline$n_{e}$ & Número de nós por elemento \\
\hline$(\xi, \eta)$ & Sistema de coordenadas padrão ou local \\
\hline$(x, y)$ & Sistema de coordenadas físicas ou global \\
\hline$v^{e}$ & Deslocamentos de um ponto qualquer do elemento $e$ \\
\hline$\hat{\boldsymbol{v}}^{e}$ & $\begin{array}{l}\text { Deslocamentos aproximados de um ponto qualquer do } \\
\text { elemento } e\end{array}$ \\
\hline$N_{i}$ & Função de forma relativa ao nó $i$ \\
\hline$U_{2 x y i}^{e}$ & $\begin{array}{l}\text { Vetor de deslocamento do nó } i \text { do elemento } e \text { (dimensão } \\
2-\operatorname{em~} x \text { e } y \text { ) }\end{array}$ \\
\hline$U_{2}^{e}$ & Vetor dos deslocamentos nodais do elemento $e$ \\
\hline
\end{tabular}




\begin{tabular}{|c|c|}
\hline símbolo & descrição \\
\hline$N_{f}$ & $\begin{array}{l}\text { Vetor das funções de forma expressas em função de } \\
\text { variáveis independentes, como as coordenadas locais } \\
-1 \leq \xi \leq 1 \text { e }-1 \leq \eta \leq 1\end{array}$ \\
\hline$\varepsilon^{e}$ & Deformações de um ponto qualquer do elemento $e$ \\
\hline$B_{2}$ & Matriz de gradiente elástico \\
\hline$B_{2 i}$ & Parte da matriz de gradiente elástico relativo ao nó $i$ \\
\hline$J$ & Matriz jacobiana \\
\hline$U$ & Energia potencial de deformação do elemento \\
\hline$W$ & $\begin{array}{l}\text { Trabalho realizado pelas tensões externas nos nós do } \\
\text { elemento }\end{array}$ \\
\hline$A^{e}$ & Área do elemento $e$ \\
\hline$\sigma^{e}$ & Tensões de um ponto qualquer do elemento $e$ \\
\hline$D_{m}$ & $\begin{array}{l}\text { Componente da matriz de elasticidade referente ao } \\
\text { material } m\end{array}$ \\
\hline$E_{m}$ & Módulo de elasticidade do material $m$ \\
\hline$\nu_{m}$ & Coeficiente de Poisson do material $m$ \\
\hline$P_{2 B}$ & Parcela das forças de volume da carga elástica $\boldsymbol{P}_{\mathbf{2}}$ \\
\hline$P_{2 S}$ & Parcela das forças de superfície da carga elástica $\boldsymbol{P}_{2}$ \\
\hline$P_{2 I}$ & Parcela das tensões iniciais da carga elástica $\boldsymbol{P}_{2}$ \\
\hline$P_{2 C}$ & $\begin{array}{l}\text { Parcela das cargas nodais concentradas da carga elástica } \\
\boldsymbol{P}_{\mathbf{2}}\end{array}$ \\
\hline$f^{B}$ & Forças de volume aplicadas externamente ao elemento \\
\hline$f^{S}$ & $\begin{array}{l}\text { Forças de superfície aplicadas externamente ao } \\
\text { elemento, em seu contorno }\end{array}$ \\
\hline$\tau_{I}^{e}$ & Tensões iniciais do elemento $e$ \\
\hline$U_{1}^{e}$ & $\begin{array}{l}\text { Vetor das variações de temperatura nodais do elemento } \\
e\end{array}$ \\
\hline$P_{2 I}^{e}$ & Vetor de tensões iniciais da carga elástica do elemento $e$ \\
\hline$\tau$ & Tensão mecânica total \\
\hline$\varepsilon$ & Deformação total \\
\hline$\varepsilon_{t}$ & Deformação térmica \\
\hline$\varepsilon_{t x}$ & Deformação térmica linear em $x$ \\
\hline$\varepsilon_{t y}$ & Deformação térmica linear em $y$ \\
\hline$\gamma_{t x y}$ & Deformação térmica angular no plano $x y$ \\
\hline$\theta$ & Temperatura num ponto do elemento \\
\hline
\end{tabular}

continua... 


\begin{tabular}{|c|c|}
\hline símbolo & descrição \\
\hline$\theta_{0}$ & Temperatura do estado livre de tensões térmicas \\
\hline$U_{1 i}^{e}$ & Variação de temperatura para cada nó $i$ do elemento $e$ \\
\hline$\tau_{x x}$ & Tensão normal em $x$ \\
\hline$\tau_{y y}$ & Tensão normal em $y$ \\
\hline$\tau_{x y}$ & Tensão de cisalhamento no plano $x y$ \\
\hline$\hat{\varepsilon}_{t}^{e}$ & $\begin{array}{l}\text { Deformação térmica aproximada num dado ponto do } \\
\text { elemento } e\end{array}$ \\
\hline$\sigma_{A}, \sigma_{B}$ & Tensões principais \\
\hline$\sigma_{v M}$ & Tensão de von Mises \\
\hline$h_{0}$ & $\begin{array}{l}\text { Coeficiente de convecção utilizado no Método de } \\
\text { Elementos Finitos }\end{array}$ \\
\hline$B_{0}$ & Matriz de gradiente elétrico \\
\hline$B_{1}$ & Matriz de gradiente térmico \\
\hline$B_{0 i}$ & Parte da matriz de gradiente elétrico relativo ao nó $i$ \\
\hline$B_{1 i}$ & Parte da matriz de gradiente térmico relativo ao nó $i$ \\
\hline$P_{1 F}$ & Parcela do fluxo de calor da carga térmica $\boldsymbol{P}_{\mathbf{1}}$ \\
\hline$P_{1 \text { conv }}$ & $\begin{array}{l}\text { Parcela das condições de fronteira de convecção da carga } \\
\text { térmica } \boldsymbol{P}_{\mathbf{1}}\end{array}$ \\
\hline$P_{1 B}$ & $\begin{array}{l}\text { Parcela de calor gerado por unidade de volume do fluxo } \\
\text { de calor } \boldsymbol{P}_{\boldsymbol{1} \boldsymbol{F}}\end{array}$ \\
\hline$P_{1 S}$ & $\begin{array}{l}\text { Parcela de fluxo de calor sobre a superfície do corpo do } \\
\text { fluxo de calor } \boldsymbol{P}_{1 \boldsymbol{F}}\end{array}$ \\
\hline$P_{1 C}$ & $\begin{array}{l}\text { Parcela de fluxo de calor nodal concentrado do fluxo de } \\
\text { calor } \boldsymbol{P}_{\mathbf{1}}\end{array}$ \\
\hline$q^{B}$ & Geração de calor interno por volume na estrutura \\
\hline$S^{e}$ & Superfície do elemento $e$ \\
\hline$q^{S}$ & Fluxo de calor através da superfície $S^{e}$ \\
\hline$S_{c}^{e}$ & $\begin{array}{l}\text { Superfície do elemento } e \text { com condições de fronteira de } \\
\text { conveç̧ão }\end{array}$ \\
\hline$\theta_{\text {conv }}$ & Vetor das variações de temperatura nodal do ambiente \\
\hline$U_{0}^{e}$ & Vetor das tensões elétricas nodais do elemento $e$ \\
\hline$P_{1 B}^{e}$ & $\begin{array}{l}\text { Vetor de geração de calor interna da carga térmica do } \\
\text { elemento } e\end{array}$ \\
\hline$j$ & Densidade de corrente (em $x$ e $y)$ \\
\hline
\end{tabular}

continua... 


\begin{tabular}{|c|c|}
\hline símbolo & descrição \\
\hline$C^{e}$ & $\begin{array}{l}\text { Propriedade qualquer do material do elemento } e \text {, } \\
\text { definido pelo modelo de interpolação de material }\end{array}$ \\
\hline$\Phi^{e}$ & $\begin{array}{l}\text { Densidade relativa de material em um elemento } e \text {, que } \\
\text { está definida no intervalo } 0 \leq \Phi^{e} \leq 1\end{array}$ \\
\hline$p$ & $\begin{array}{l}\text { Fator de penalização da variável de projeto no modelo } \\
\text { de material SIMP }\end{array}$ \\
\hline$C_{m}$ & Propriedade qualquer do material $m$ \\
\hline$E_{\text {vazio }}$ & Módulo de elasticidade do vazio \\
\hline$K$ & $\begin{array}{l}\text { Rigidez da peça atuada pelo deslocamento de saída do } \\
\text { mecanismo }\end{array}$ \\
\hline$u_{\text {saida }}$ & Deslocamento de saída do mecanismo \\
\hline$F_{E T M}$ & $\begin{array}{l}\text { Função objetivo da otimização de um MEMS } \\
\text { eletrotermomecânico }\end{array}$ \\
\hline$G_{v^{\prime} l_{m}}$ & $\begin{array}{l}\text { Função que define o volume de um material } m \text { na } \\
\text { topologia }\end{array}$ \\
\hline$\Phi_{m}$ & $\begin{array}{l}\text { Densidade relativa de um material } m \text { (entre zero e um) } \\
\text { de cada ponto do domínio fixo estendido }\end{array}$ \\
\hline$\Omega_{s_{m}}$ & Volume máximo permitido para um material $m$ \\
\hline$M$ & $\begin{array}{l}\text { Número de materiais, além do vazio, considerado na } \\
\text { análise }\end{array}$ \\
\hline$N_{\text {carga }}$ & Número de casos de carga do mecanismo multiflexível \\
\hline$u_{\text {said }_{c}}$ & $\begin{array}{l}\text { Deslocamento de saída do mecanismo multiflexível para } \\
\text { o caso de carga } c\end{array}$ \\
\hline$F_{E T M_{m u l t i}}$ & $\begin{array}{l}\text { Função objetivo da otimização de um MEMS } \\
\text { eletrotermomecânico multiflexível }\end{array}$ \\
\hline$\psi$ & $\begin{array}{l}\text { Parâmetro de normalização da função Kreisselmeier- } \\
\text { Steinhauser (KS) }\end{array}$ \\
\hline$\Phi_{A}^{e}$ & $\begin{array}{l}\text { Densidade relativa entre sólido (composto) e vazio no } \\
\text { modelo de material SIMP estendido }\end{array}$ \\
\hline$\Phi_{B}^{e}$ & $\begin{array}{l}\text { Densidade relativa entre o material } 1 \text { e } 2 \text {, na parte sólida, } \\
\text { no modelo de material SIMP estendido }\end{array}$ \\
\hline$C_{v a z i o}$ & Propriedade qualquer do vazio \\
\hline$\mu_{m}$ & $\begin{array}{l}\text { Média da distribuição de Gauss do termo referente ao } \\
\text { material } m \text {, no modelo de material de função de pico }\end{array}$ \\
\hline
\end{tabular}

continua... 


\begin{tabular}{|c|c|}
\hline símbolo & descrição \\
\hline$\sigma_{m}$ & $\begin{array}{l}\text { Desvio padrão da distribuição de Gauss do termo } \\
\text { referente ao material } m \text {, no modelo de material de } \\
\text { função de pico }\end{array}$ \\
\hline$L_{2}$ & Vetor que mascara o deslocamento de saída do restante \\
\hline$\Lambda_{i}$ & Vetores adjuntos $(i=0,1$ ou 2$)$ \\
\hline$K_{i}^{*}$ & $\begin{array}{l}\text { Matriz } K_{i}(i=0,1 \text { ou } 2) \text { modificada de acordo com a } \\
\text { aplicação de condições de contorno de Dirichlet }\end{array}$ \\
\hline$\Lambda_{i}^{*}$ & $\begin{array}{l}\text { Vetores adjuntos considerando condições de contorno de } \\
\text { Dirichlet }(i=0,1 \text { ou } 2)\end{array}$ \\
\hline$U_{1 i}^{e}$ & Grau de liberdade térmico $i$ do elemento $e$ \\
\hline$U_{0 i}^{e}$ & Grau de liberdade elétrico $i$ do elemento $e$ \\
\hline$\Phi_{m}^{e}$ & $\begin{array}{l}\text { Densidade relativa de um material } m \text { (entre zero e um) } \\
\text { do elemento } e\end{array}$ \\
\hline$V^{e}$ & Volume do elemento $e$ \\
\hline$F_{O b j}$ & $\begin{array}{l}\text { Função objetivo, que pode ser tanto } F_{E T M} \text {, como } \\
F_{E T M_{\text {multi }}}\end{array}$ \\
\hline$F_{O b j}^{l i n e a r}$ & Função objetivo $F_{O b j}$ linearizada \\
\hline$\rho_{0}$ & Valor de $\boldsymbol{\rho}$ em torno do qual a linearização é realizada \\
\hline$\rho_{0}^{e}$ & Pseudo-densidade do elemento $e$ de $\rho_{0}$ \\
\hline$G_{v^{\prime i n e a r}}^{\text {liner }}$ & Função $G_{v o l_{m}}$ linearizada \\
\hline$\rho_{\max }^{e}$ & Limite móvel superior do elemento $e$ \\
\hline$\rho_{\text {min }}^{e}$ & Limite móvel inferior do elemento $e$ \\
\hline$\omega_{D P_{m}}$ & $\begin{array}{l}\text { Coeficiente de continuação do desvio padrão de cada } \\
\text { material } m\end{array}$ \\
\hline $\begin{array}{l}\Omega_{s_{m f i n a l}} \\
\omega_{R V_{m}}\end{array}$ & $\begin{array}{l}\text { Valor final do volume máximo de cada material } m \\
\text { Coeficiente de continuação do volume máximo do } \\
\text { material } m\end{array}$ \\
\hline$R_{\text {filtro }}$ & Raio de ação o filtro espacial \\
\hline$\rho_{\text {lim }}^{e}$ & $\begin{array}{l}\text { Limite móvel (inferior ou superior) da variável de projeto } \\
\rho^{e}, \text { referente ao elemento } e\end{array}$ \\
\hline$\rho_{l i m}^{f}$ & $\begin{array}{l}\text { Limite móvel (inferior ou superior) da variável de projeto } \\
\rho^{f} \text {, referente ao elemento } f\end{array}$ \\
\hline$V^{f}$ & Volume do elemento $f$ \\
\hline$n f$ & $\begin{array}{r}\text { Número de elementos } f \text { considerados pelo filtro espacial } \\
\text { continua... }\end{array}$ \\
\hline
\end{tabular}




\begin{tabular}{ll}
\hline símbolo & descrição \\
\hline$R^{f}$ & $\begin{array}{l}\text { Distância medida entre os centróides dos elementos } e \\
\text { (referência para o filtro espacial) e } f \text { (dentro do raio de } \\
\end{array}$ \\
& ação do filtro) \\
$T_{a m b}$ & Temperatura ambiente \\
$I_{n d A c o p l}$ Índice de acoplamento do caso de carga $c$ \\
$q^{c}$ & Fluxo de calor nodal concentrado \\
\hline
\end{tabular}




\section{Introdução}

\subsection{MEMS Eletrotermomecânicos}

Um micromecanismo é essencialmente um dispositivo de dimensões milimétricas ou até micrométricas que executa uma tarefa específica como atuar como garra, pinça, grampo, etc. (ISHIHARA; ARAI; FUKUDA, 1996) e gera deslocamentos da ordem de centenas de nanômetros até micrômetros. Quando acoplados a um sistema eletrônico, são chamados de sistemas microeletromecânicos ou "MicroElectro-Mechanical Systems" (MEMS). Esses micromecanismos são fabricados utilizando-se técnicas de litografia e processos de microfabricação de superfícies usados nas indústrias de semicondutores (PETERSEN, 1982; RAI-CHOUDHURY, 2000).

As aplicações dessa tecnologia estão relacionadas principalmente com a área de Mecânica de Precisão e Mecatrônica (ISHIHARA; ARAI; FUKUDA, 1996; REYNAERTS; PEIRS; BRUSSEL, 1998). São exemplos de aplicações: mecanismo de leitura de disco rígido de computadores e cabeçotes de videocassete; mecanismos internos em máquinas fotográficas; antenas de celulares; MEMS (RAICHOUDHURY, 2000); instrumentos de microcirurgia (microgarras, micropinças); instrumentos de microscopia eletrônica; posicionadores de lentes em sistemas de interferometria laser; equipamentos para manipulação de células; nanometrologia; equipamentos destinados à manipulação em nanotecnologia (manipulação de nanotubos, por exemplo); equipamentos de mecânica de precisão em geral, etc. Portanto, consiste numa tecnologia cuja aplicação vem crescendo no mundo atual.

Os investimentos em pesquisa, aplicações industriais e interesse científico na área de MEMS têm crescido consideravelmente no mundo nos últimos sete anos. Vários artigos sobre o projeto e fabricação de MEMS podem ser encontrados na literatura (JONSMANN, 1999; REYNAERTS; PEIRS; BRUSSEL, 1998). No Brasil, sua fabricação tem sido tema de interesse e estudo de grupos de pesquisa como o Laboratório de Sistemas Integráveis (LSI) do Departamento de Engenharia Eletrônica da Escola Politécnica da USP e o Laboratório de Microfabricação 
(LMF) no Laboratório Nacional de Luz Síncrotron (LNLS), em Campinas-SP.

Em geral, os MEMS podem ser atuados de três formas: capacitiva, eletrotermomecânica e piezelétrica. Todas elas permitem que o dispositivo seja atuado por meio da aplicação de um potencial elétrico. A atuação capacitiva apresenta a desvantagem da não-linearidade entre a voltagem aplicada e o deslocamento gerado. A atuação piezelétrica apresenta linearidade entre a voltagem aplicada e o deslocamento gerado, entretanto tem como principal desvantagem a dificuldade tecnológica de se depositar o material piezelétrico na escala do MEMS. Já a atuação eletrotermomecânica tem como vantagem a linearidade entre a voltagem aplicada e o deslocamento gerado, mas apresenta um tempo de resposta maior do que as anteriores, sendo muito utilizada na atuação dos MEMS (ISHIHARA; ARAI; FUKUDA, 1996; JONSMANN, 1999; RAICHOUDHURY, 2000).

Neste trabalho são estudados MEMS com atuação eletrotermomecânica. Os MEMS eletrotermomecânicos convertem uma entrada elétrica numa saída mecânica (ANANTHASURESH, 2003; YIN; ANANTHASURESH, 2002; SIGMUND, 2001b, 2001c). Assim, uma corrente elétrica é convertida em calor, pelo efeito Joule, que causa tensões térmicas, originando uma deformação estrutural do mecanismo (MANKAME; ANANTHASURESH, 2001).

Para poder gerar movimentos que envolvam deslocamentos da ordem de centenas de nanômetros até micrômetros, esses micromecanismos são essencialmente constituídos pelos chamados mecanismos flexíveis (HOWELL, 2001). Um mecanismo flexível é um mecanismo, geralmente de geometria complexa, para o qual o movimento é dado pela flexibilidade de uma estrutura, ao invés de ser pela presença de juntas e pinos (KOTA et al., 2000). Tem como principal vantagem o fato de consistir numa única peça, o que implica em: inexistência de problemas de folga devido à montagem de pinos (isto inviabilizaria a transmissão de deslocamentos da ordem de grandeza exigida em micromanipulação), descarte da necessidade de montagem (o que por si só já seria um problema) e o fato de não necessitar de lubrificação, pois não há atrito entre peças. As principais desvantagens são o problema de fadiga e o fato da operação se limitar, em geral, a pequenos deslocamentos.

Mecanismos flexíveis possuem várias aplicações, mas certamente uma área em que a sua utilização tem contribuído bastante é na área de MEMS. Na microescala em que são fabricados estes MEMS, a presença de pinos e juntas torna a montagem difícil ou impossível e a presença de folgas não permitiria 


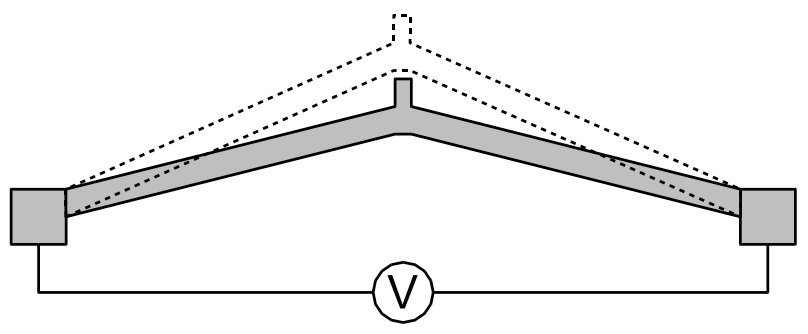

Figura 1.1: Exemplo de um MEMS eletrotermomecânico do tipo "V" aberto e sua forma sob atuação (em pontilhado).

transmitir deslocamentos da ordem de nano a micrômetros gerados (KOTA et al., 2000). Assim, as características técnicas dos mecanismos flexíveis, como descritas anteriormente, se enquadram perfeitamente dentro dos requisitos para aplicação em sistemas microeletromecânicos e, hoje, praticamente todos esses projetos utilizam mecanismos flexíveis. Além disso, as técnicas de microfabricação de MEMS permitem a obtenção de estruturas planas de forma complexa (RAICHOUDHURY, 2000).

Dentre os MEMS eletrotermomecânicos existentes e projetados de forma intuitiva, dois tem maior destaque na literatura: MEMS no formato de um "V" aberto (QUE; PARK; GIANCHANDANI, 1999; PARK et al., 2000; CHU; GIANCHANDANI, 2003; CHU et al., 2003) e os pseudo-bilaminares (CHEN; KUNG; LEE, 2002; MOULTON; ANANTHASURESH, 2001; COMTOIS; MICHALICEK; BARRON, 1998). Os microatuadores no formato de um "V" aberto caracterizamse pelo movimento no plano uniaxial, e são comumente fabricados em silício policristalino e em níquel eletrodepositado (ver exemplo na figura 1.1). Os MEMS pseudo-bilaminares são caracterizados pelo movimento em arco. São microatuadores que imitam o princípio de funcionamento dos atuadores bilaminares (que empregam dois materiais com coeficientes de expansão térmica diferentes para realizar o movimento) através do emprego de áreas de seções diferentes no mesmo atuador. Conforme a figura 1.2, pelo fato da parte inferior ter espessura menor que a superior a corrente irá provocar um aquecimento maior na parte inferior originando a atuação como mostrado. $\mathrm{O}$ micromecanismo pseudobilaminar também pode ser empregado com movimentação uniaxial, próprio dos microatuadores ETM no formato de "V" aberto, o que é mostrado por Moulton e Ananthasuresh (2001) e Syms (1998). Um exemplo de tal mecanismo encontra-se na figura 1.3. No caso deste último mecanismo, as resistências elétricas das seções maior e menor estão em paralelo, o que acaba por permitir uma maior passagem de corrente na seção maior, onde ocorre o maior aquecimento e dilatação térmica.

Enfocando a modelagem térmica dos MEMS eletrotermomecânicos, Lerch et 


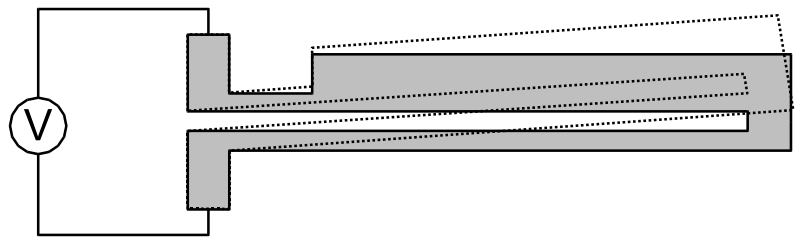

Figura 1.2: Exemplo de um MEMS eletrotermomecânico pseudo-bilaminar e sua forma sob atuação (em pontilhado).

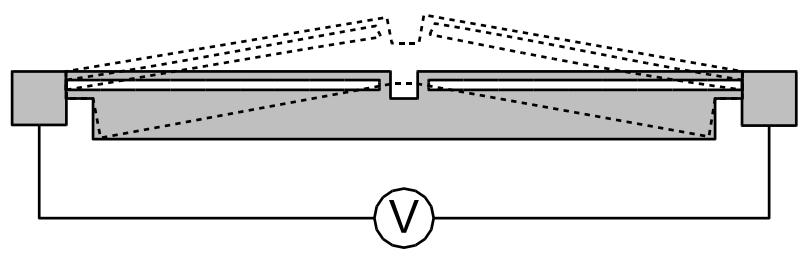

Figura 1.3: Exemplo de um MEMS eletrotermomecânico pseudo-bilaminar uniaxial e sua forma sob atuação (em pontilhado).

al. (1996) realizaram uma análise usando o Método de Elementos Finitos (MEF) de uma garra baseada neste tipo de microatuadores, para uma temperatura máxima no MEMS de 800K. As simulações em MEF produziram uma distribuição de temperatura que concorda em $90 \%$ com a distribuição de temperaturas obtidas mediante um termômetro óptico. Eles também modelaram e caracterizaram microatuadores térmicos assimétricos. Mankame e Ananthasuresh (2001), Yan, Khajepour e Mansour (2003) realizaram um estudo sobre a modelagem térmica em microsistemas considerando microatuadores eletrotermomecânicos no formato pseudo-bilaminar, onde mostrou-se que fatores como a radiação entre as partes do micromecanismo (particularmente em temperaturas superiores a $500 \mathrm{~K}$ ), a conveç̧ão natural e as perdas de calor da estrutura pelo substrato são significativos para se ter um modelo computacional consistente com a realidade.

Apesar da maioria dos artigos em projeto de micromecanismos eletrotermomecânicos abordar o regime estático, Comtois e colaboradores (COMTOIS; MICHALICEK; BARRON, 1998; REID; BRIGHT; COMTOIS, 1996) caracterizaram o desenvolvimento de MEMS eletrotermomecânicos em estado estacionário e dinâmico e têm proposto parâmetros para o seu projeto. Hickey et al. (2003) publicaram um estudo sobre a resposta de freqüência deste tipo de MEMS eletrotermomecânicos.

Outro aspecto fora do escopo tradicional da análise estática é a falha desses mecanismos. Que et al. (2000) realizaram estudos quanto ao número de ciclos e a influência do projeto e condições de operação nos microatuadores eletrotermomecânicos no formato de um "V" aberto, e também estabeleceu para eles um modelo de falha catastrófica em operação contínua e pulsada (QUE; PARK; 


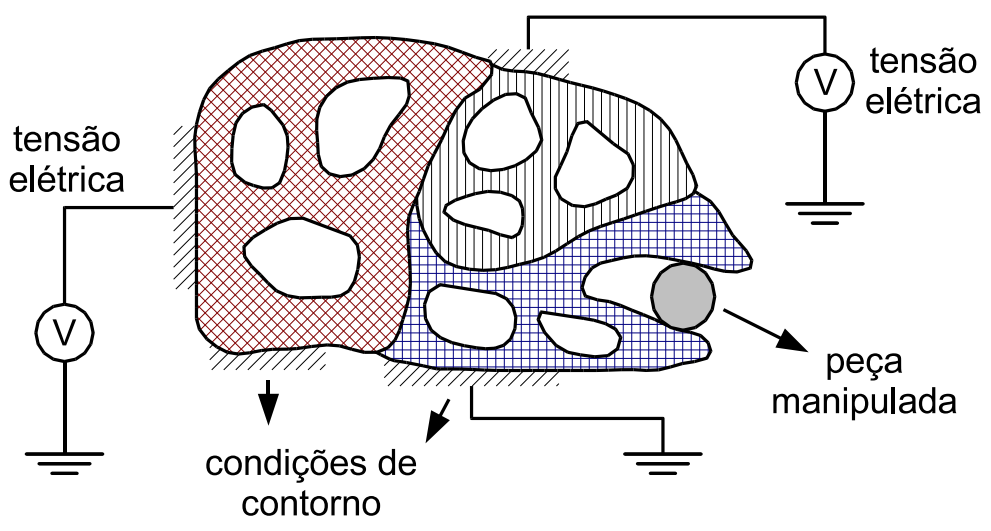

Figura 1.4: Exemplo de um micromecanismo multifásico (com dois ou mais materiais) multi-atuado, com condições de contorno mecânicas (engastamento) e elétricas.

GIANCHANDANI, 2001; QUE et al., 2001).

\subsection{O problema do projeto de MEMS eletrotermomecânicos}

Embora as técnicas de processo de fabricação de micromecanismos tenham atingido um alto nível de maturidade, a modelagem e, em particular, o desenvolvimento de métodos computacionais sistemáticos para o projeto de MEMS estão ainda no seu estágio inicial (LARSEN; SIGMUND; BOUWSTRA, 1997).

O projeto genérico de um micromecanismo é uma tarefa complexa. Essencialmente, deseja-se projetar um mecanismo flexível que execute diferentes tarefas quando sujeito a diferentes atuações, o que envolve o conceito de multiflexibilidade (NISHIWAKI et al., 2001), segundo o qual o micromecanismo deve realizar diferentes tarefas (avanço em $x$ ou em $y$, ou abrir e fechar a garra) dependendo da atuação (força) aplicada. Além disso, pode-se explorar a utilização de dois, ou até mais, materiais. Os dois conceitos (multiflexibilidade e utilização de múltiplos materiais) são ilustrados na figura 1.4.

Apesar de ser possível projetar mecanismos eletrotermomecânicos bidimensionais flexíveis com vários graus de liberdade de saída utilizando apenas um material, a possibilidade de se usar um outro material na estrutura pode aumentar o desempenho e funcionalidade do microatudor (SIGMUND, 2001c), sendo essa a principal motivação em se projetar micromecanismos multifásicos. Como por exemplo, um mecanismo flexível com dois materiais pode sofrer deformações maiores, e com isso promover deslocamentos maiores, sem que seja excedido o limite de resistência do material (YIN; ANANTHASURESH, 


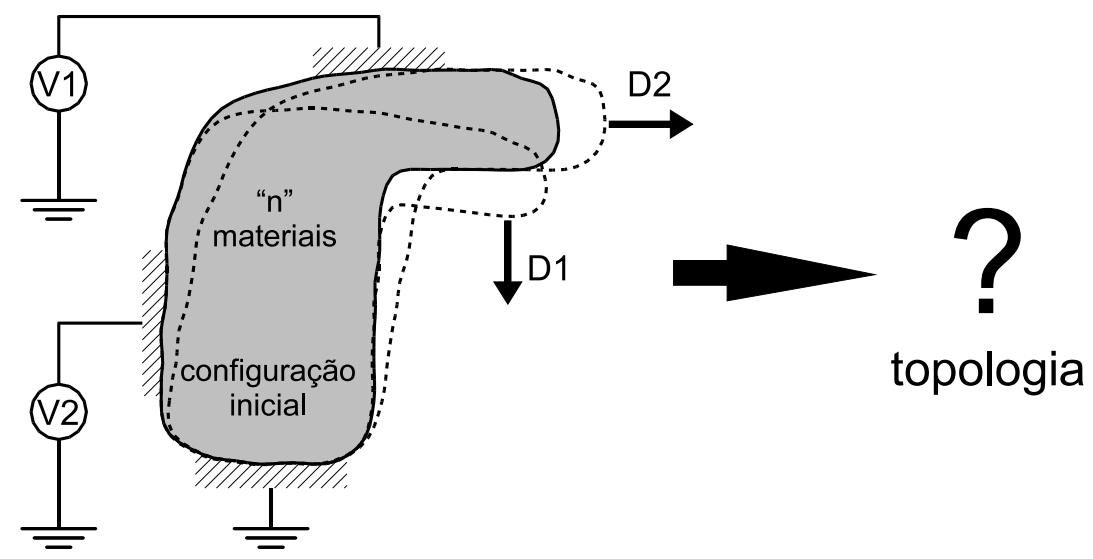

Figura 1.5: Projeto de um micromecanismo multifásico multiflexível. Cada tensão elétrica $\mathrm{V}$ gera o respectivo deslocamento de interesse $\mathrm{D}$, de forma separada e independente.

2001). No caso do mecanismo de vários graus de liberdade, pode-se usar um material adicional que não conduza eletricidade, o que permitiria isolar algumas partes da estrutura e obter mais facilmente atuadores com pouco acoplamento entre os graus de liberdade (SIGMUND, 2001c). Por outro lado, têm-se também como motivação o fato de que estão emergindo técnicas de microfabricação que permitem fabricar peças heterogêneas (materiais diferentes) sem montagem e com interfaces resistentes entre os materiais (YIN; ANANTHASURESH, 2001). O projeto de micromecanismos com vários materiais já vem sendo estudado por vários grupos de pesquisas no mundo (SIGMUND, 2001c; YIN; ANANTHASURESH, 2001, 2002). A idéia de projeto, o qual envolve a multiflexibilidade e existência de mais de um material, além do vazio, pode ser vista na figura 1.5.

Assim, o projeto de micromecanismos exige conhecimentos multidisciplinares em estruturas e projeto de mecanismos flexíveis, sendo que a utilização de dois ou mais materiais aumenta ainda mais a complexidade deste tipo de projeto. Devido a essa complexidade e à falta de métodos sistemáticos, o projeto de micromecanismos vem sendo realizado por métodos de tentativa e erro, com auxílio da intuição e experiência do projetista (ISHIHARA; ARAI; FUKUDA, 1996; REYNAERTS; PEIRS; BRUSSEL, 1998). Desta forma, uma das áreas de grande contribuição científica no segmento destes microdispositivos, atualmente, é a de desenvolvimento de métodos computacionais de simulação e métodos de projeto sistemáticos como o Método de Otimização Topológica (MOT). Este último permite a exploração da tecnologia de micromecanismos e estruturas com dois ou até mais materiais (SIGMUND, 2001c; WANG; WANG, 2004, 2005; YIN; ANANTHASURESH, 2001, 2002; YULIN; XIAOMING, 2004) e tem muito a contribuir para melhorar, consideravelmente, os projetos existentes e para fornecer novos e mais eficientes microdispositivos, ou microdispositivos que possam realizar 
diferentes tarefas em diferentes aplicações.

\subsection{Método de Otimização Topológica aplicado ao projeto de MEMS eletrotermomecânicos}

O Método de Otimização Topológica (MOT) implementado nesse trabalho combina essencialmente métodos de otimização com um método numérico de análise, como por exemplo, o MEF (BATHE, 1995). Outros métodos numéricos de análise podem ser usados. No entanto, devem ser genéricos o suficiente de forma a lidar com estruturas de formas complexas resultantes da otimização. Os métodos de otimização aceleram o processo de busca da distribuição ótima de material, utilizando-se para isso da informação dos gradientes (ou derivadas) da função custo em relação à quantidade de material em cada elemento (HAFTKA; GURDAL; KAMAT, 1990).

Desde sua introdução, o MOT vem ganhando destaque no meio acadêmico e na indústria. Torna o processo de projeto mais genérico, sistemático, otimizado, e independente da experiência específica de alguns engenheiros, fornecendo a topologia inicial, otimizada para uma certa aplicação do dispositivo a ser construído. A presença do engenheiro é necessária para a obtenção do projeto final e verificação do desempenho para o qual foi projetado (o que é feito com o auxílio de métodos numéricos e experimentais).

Originalmente foi desenvolvido para projetos de maximização da rigidez de estruturas e tem sido expandido, recentemente e com sucesso, para atuação em várias outras aplicações, sejam elas multifísicas (como o projeto de MEMS eletrotermomecânicos) ou não. Algumas dessas aplicações são o projeto de: microestruturas de material com propriedades termoelásticas extremas (SIGMUND; TORQUATO, 1996, 1997); microestruturas de materiais piezelétricos (SILVA; FONSECA; KIKUCHI, 1998; SIGMUND; TORQUATO; AKSAY, 1998); microestruturas tridimensionais com rigidez extrema e limites sobre a condutividade (SIGMUND, 1999); atuadores piezoelétricos (SILVA; NISHIWAKI; KIKUCHI, 2000; CHEN; SILVA; KIKUCHI, 2001); antenas e motores eletromagnéticos (YOO; KIKUCHI, 2000); microestruturas de cristal fotônico (JENSEN; SIGMUND, 2004); problemas eletromagnéticos (BYUN; LEE; PARK, 2004); motores de indução para compressores rotativos (WANG; KANG; NOH, 2004). Já trabalhos preliminares desenvolvidos utilizando o MOT envolvendo tópicos relacionados a MEMS eletrotermomecânicos são: estruturas carregadas termicamente (RODRIGUES; 
FERNANDEZ, 1995); atuadores térmicos (SIGMUND, 1997); projeto de mecanismos flexíveis (SIGMUND, 1997; NISHIWAKI et al., 1998; LIMA; SILVA, 2001); projeto de mecanismos de microgarras e micropinças (LARSEN; SIGMUND; BOUWSTRA, 1997).

As primeiras aplicações do MOT no projeto de MEMS foram desenvolvidas independentemente para o projeto de mecanismos flexíveis por Ananthasuresh, Kota e Gianchandani (1994), Sigmund (1996) e os primeiros trabalhos que abordaram o projeto de MEMS eletrotermomecânicos utilizando o MOT foram publicados por Sigmund (2001b, 2001c). Sigmund (2001c) e Yin e Ananthasuresh (2001) desenvolveram trabalhos que envolveram o MOT aplicado ao projeto de MEMS eletrotermomecânicos multifásicos. Sigmund (2001c) estendeu seu próprio trabalho para que considerasse dois materiais, além do vazio, no projeto otimizado. O acréscimo de um material na análise levou ao aumento do número de variáveis de projeto, o que não acontece ao se utilizar a metodologia proposta por Yin e Ananthasuresh (2001). Esses trabalhos, assim como outros que utilizam o MOT no projeto de estruturas multifásicas, são apresentados com mais detalhes na seção 3.3.2.

Uma das principais características a serem otimizadas é o deslocamento dos MEMS. Chen, Kung e Lee (2002) discutiram a geometria ótima de um micromecanismo eletrotermomecânico pseudo-bilaminar para maximizar o deslocamento. Fazendo um paralelo com o projeto de mecanismos flexíveis, a simples maximização do deslocamento numa direção de um dado ponto do mecanismo acaba por gerar a topologia de maior flexibilidade (YIN; ANANTHASURESH, 2002), podendo torná-la ineficaz para o seu propósito. Assim, quando se deseja o maior deslocamento do mecanismo projetado, existem duas abordagens principais utilizadas para contornar esse problema. Uma aproximação iniciada por Sigmund (1997), e mantida em seus trabalhos subseqüentes (SIGMUND, 2001b, 2001c), na qual o carregamento de saída é aproximado por uma mola no ponto e direção do deslocamento de saída, foi seguida por diversos autores como: Larsen, Sigmund e Bouwstra (1997), Hetrick, Kikuchi e Kota (1999), Jonsmann, Sigmund e Bouwstra (1999b, 1999a), Canfield e Frecker (2000), Tai e Chee (2000), Lau, Du e Lim (2001a, 2001b), Pedersen (2001), Bruns e Tortorelli (2001, 2003), Yin e Ananthasuresh (2001). A outra abordagem está baseada na maximização da razão de duas energias mútuas baseadas em dois diferentes problemas de elementos finitos como proposto por Frecker et al. (1997). Os autores que seguiram esta aproximação foram: Nishiwaki et al. (1998), Frecker, Kikuchi e Kota (1999), Kota et al. (2001), Ejima et al. (2000), 
Nishiwaki et al. (2001), Chen, Silva e Kikuchi (2001). Segundo Hetrick, Kikuchi e Kota (1999), os problemas que utilizam a aproximação do carregamento de saída por uma mola apresentam menos problemas de convergência, em comparação à abordagem da energia mútua, e não tem dependência do limite mínimo da densidade do elemento. Ao contrário da abordagem da energia mútua, o uso de mola como carregamento de saída é facilmente implementado tanto em projetos de mecanismos flexíveis como de mecanismos eletrotermomecânicos e será usado neste trabalho.

\subsection{Objetivo e Justificativa}

Nesse trabalho, pretende-se estudar e implementar o MOT para o projeto de MEMS eletrotermomecânicos multifásicos que sejam multiflexíveis, ou seja, realizem diferentes tarefas, considerando-se diferentes atuações elétricas. O objetivo é desenvolver um programa de computador ("software"), que possa ser utilizado por engenheiros em diferentes áreas, para o projeto desses micromecanismos, considerando uma dada aplicação desejada. Entre os métodos usados até agora para o projeto de MEMS eletrotermomecânicos, o MOT tem obtido o maior sucesso por ser genérico e sistemático (SIGMUND, 2001b), além de ser bem conhecida no grupo de pesquisa do aluno.

Esse tema se justifica pelo fato do desenvolvimento de métodos computacionais sistemáticos para o projeto de MEMS estar ainda no seu estágio inicial, ao contrário do relativamente alto nível de maturidade das técnicas de processo de fabricação de micromecanismos. O projeto genérico de um micromecanismo é uma tarefa complexa, ainda mais quando este envolve análises de diferentes naturezas (elétrica, térmica e elástica), utiliza dois ou mais materiais, além do vazio, e executa diferentes tarefas quando sujeito a diferentes atuações.

O projeto destes MEMS é considerado um problema de otimização estrutural, no qual se deseja maximizar um determinado deslocamento numa dada direção e ponto da estrutura, dada certa excitação térmica na estrutura (causada pela aplicação de uma corrente ou tensão elétrica, por exemplo), restringindo o volume total dos materiais empregados na fabricação do mecanismo. 


\section{Modelagem de MEMS Eletrotermomecânicos pelo MEF}

Como é típico em MEMS, os microatuadores eletrotermomecânicos operam em diversos domínios de energia, em particular nos domínios elétrico, térmico e elástico. Portanto, a simulação do comportamento deste tipo de micromecanismo envolve três etapas de análises: a análise elétrica, para determinar a distribuição de tensões no MEMS quando se aplica uma corrente; a análise térmica, para determinar a distribuição de temperatura devido ao efeito Joule; e a análise de deformação elástica gerada pela presença de cargas térmicas. Assim, no princípio de atuação eletrotermomecânica uma corrente elétrica é convertida em calor gerado pelo efeito Joule, o calor produzido induz tensão térmica, que causa uma deformação elástica.

Inicialmente, serão discutidas as equações governantes para uma estrutura geral carregada eletrotermomecanicamente, assim como a representação destas equações utilizando o Método de Elementos Finitos (MEF), descrito adiante. A seguir, apresenta-se uma descrição sobre o elemento retangular de quatro nós utilizado na discretização do MEF e sobre a sua formulação teórica para cada domínio físico envolvido nos micromecanismos eletrotermomecânicos: elétrico e térmico, e elástico. Ao longo do texto, as variáveis de projeto foram consideradas por elemento.

\subsection{Equações governantes dos micromecanismos eletrotermomecânicos}

Por razões de manufatura, a maior parte dos microatuadores eletrotermomecânicos são modelados e simulados como estruturas bidimensionais. Para efeitos de projeto otimizado, pode-se simplificar o problema assumindo que atuam de forma linear no caso de pequenas deformações e o material apresenta 


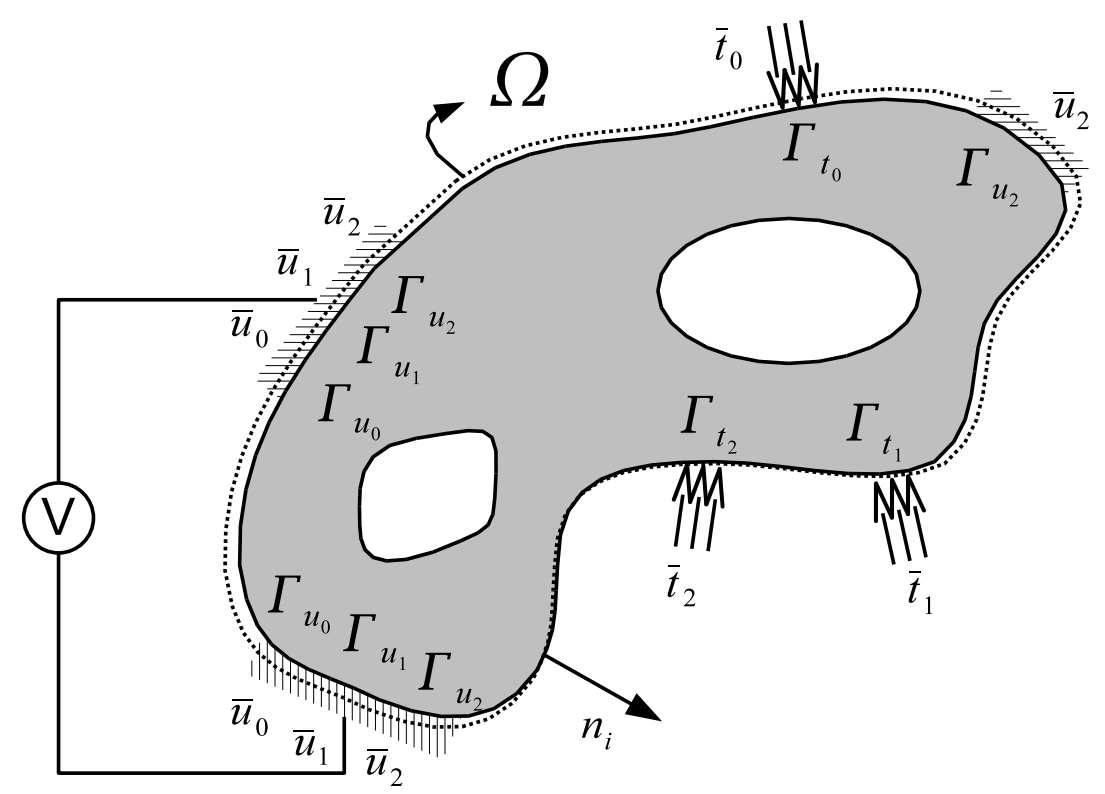

Figura 2.1: Condições de carga e fronteira para o problema eletrotermomecânico.

comportamento linear. As pequenas deformações permitem que os campos elétrico e mecânico possam ser modelados usando hipótese linear e, deste modo, o sistema é apenas fracamente acoplado, no sentido que as equações de calor não dependem das equações de elasticidade e que as equações do campo elétrico são independentes das equações de calor (ANANTHASURESH, 2003; MANKAME; ANANTHASURESH, 2001; SIGMUND, 2001b).

Considerando o sólido mostrado na figura 2.1, a estrutura a ser analisada é um subdomínio do domínio $\Omega$, o qual contém material linear termoeletroelástico. As fronteiras estão compostas de seis pares de regiões desligadas $\Gamma=\overline{\Gamma_{u_{0}} \cup \Gamma_{t_{0}}}=$ $\overline{\Gamma_{u_{1}} \cup \Gamma_{t_{1}}}=\overline{\Gamma_{u_{2}} \cup \Gamma_{t_{2}}}$, onde os índices 0,1 e 2 referem-se às condições de fronteira elétrica, térmica e mecânica, respectivamente, e os subíndices $u$ e $t$ referem-se às condições de fronteira de deslocamento e de cargas superficiais, respectivamente. As regiões $\Gamma_{u_{i}}$ podem coincidir em parte superpostas ou podem ser completamente separadas, o que aplica para as regiões $\Gamma_{t_{i}}$ também.

As equações para os domínios elétrico, eletrotérmico e termoelástico, respectivamente, são:

- Domínio elétrico

$$
\begin{aligned}
\sigma_{0} u_{0, i i}+b_{0} & =0 & & \text { em } \Omega \\
u_{0} & =\bar{u}_{0} & & \text { sobre } \Gamma_{u_{0}} \\
-\sigma_{0} u_{0, n} & =\bar{t}_{0} & & \text { sobre } \Gamma_{t_{0}}
\end{aligned}
$$


- Domínio eletrotérmico

$$
\begin{aligned}
\sigma_{1} u_{1, i i}+b_{1} & =0 & & \text { em } \Omega \\
b_{1} & =-\sigma_{0} u_{0, i}^{2}-h\left(\bar{u}_{1 \infty}-u_{1}\right) & & \\
u_{1} & =\bar{u}_{1} & & \text { sobre } \Gamma_{u_{1}} \\
-\sigma_{1} u_{1, n} & =\bar{t}_{1} & & \text { sobre } \Gamma_{t_{1}}
\end{aligned}
$$

- Domínio termoelástico

$$
\begin{array}{rlrl}
s_{i j, j}+b_{2(i)} & =0 & & \text { em } \Omega \\
s_{i j} & =E_{i j k l}\left(\eta_{k l}-\alpha_{k l} u_{1}\right) & \text { em } \Omega \\
\eta_{i j} & =\frac{1}{2}\left(u_{2(i, j)}+u_{2(j, i)}\right) & \text { em } \Omega \\
u_{2(i)} & =\bar{u}_{2(i)} & \text { sobre } \Gamma_{u_{2}} \\
s_{i j} n_{j} & =\bar{t}_{2(i)} & & \text { sobre } \Gamma_{t_{2}}
\end{array}
$$

onde as variáveis $u$ são variáveis de estado, sendo $u_{0}$ a tensão elétrica existente, $u_{1}$ a variação de temperatura em relação ao estado livre de tensões térmicas e $u_{2(i)}$ o componente do deslocamento na direção $i$. A notação ${ }_{i}$ significa diferenciação com respeito à direção da coordenada $i$. $b_{0}$ é a fonte prescrita de corrente interna por unidade de volume, $h$ o coeficiente de convecção, $b_{1}$ modela a geração interna de calor por unidade de volume e a convecção da superfície superior, $b_{2(i)}$ são as componentes de força no corpo. $\sigma_{0}$ e $\sigma_{1}$ são as condutividades elétrica e térmica (isotrópica), respectivamente. $E_{i j k l}$ é o tensor de rigidez elástica. $\bar{u}_{0}$ e $\bar{u}_{1}$ são a tensão elétrica e a variação de temperatura prescrita, respectivamente. $\bar{u}_{1 \infty}$ é a variação de temperatura do ambiente. $\bar{u}_{2(i)}$ são as componentes de deslocamentos prescritas. $\bar{t}_{0}$ e $\bar{t}_{1}$ são o fluxo de corrente e calor prescritos, respectivamente, e $\bar{t}_{2(i)}$ são as componentes de força de tração. $\eta_{i j}, s_{i j}$ e $\alpha_{i j}$ são os tensores de deformações de engenharia, de tensões mecânicas e de expansão térmica, respectivamente. $n_{i}$ são as componentes do vetor normal unitário para a superfície $\Gamma$. A geração de calor interna $b_{1}$ é uma função do campo elétrico $u_{0}$ e a tensão $s_{i j}$ é uma função do campo de temperatura $u_{1}$ (ANANTHASURESH, 2003; MANKAME; ANANTHASURESH, 2001; SIGMUND, 2001b). 


\subsection{Formulação do Método de Elementos Finitos}

No caso do estudo dos micromecanismos eletrotermomecânicos, assim como de qualquer sistema de Engenharia, é necessário desenvolver um modelo matemático que descreva esse sistema, o qual implica assumir algumas hipóteses que simplifiquem a expressão matemática governante da conduta dos sistemas, mas, usualmente, essas expressões consistem de equações diferenciais como as descritas na seção anterior.

Essas equações diferenciais são difíceis de resolver analiticamente, mas com o advento da tecnologia computacional, torna-se viável resolver essas equações governantes. Varias técnicas de solução numérica tem sido desenvolvidas e aplicadas em númerosos problemas de Engenharia para encontrar soluções aproximadas. Especialmente o MEF tem sido uma das técnicas de maior sucesso. Uma das maiores vantagens do MEF é a sua fácil implementação computacional para resolver e analisar diversos tipos problemas físicos, sejam acoplados ou não, dividindo o domínio do problema em muitos subdomínios de fácil análise e onde cada subdomínio recebe o nome de elemento finito. Em particular, qualquer forma complexa do domínio de projeto com condições de fronteira prescritas pode ser manipulada com facilidade usando o MEF. Este método é utilizado na Otimização Topológica (OT) por ser um método de análise robusto, confiável e genérico o suficiente para lidar com estruturas de formas complexas resultantes deste tipo de otimização. Ainda que outros métodos de análise possam ser usados, o MEF é a técnica mais comum em todos os trabalhos envolvendo o MOT consultados na literatura (ANANTHASURESH, 2003; BENDSøE; SIGMUND, 2002; SIGMUND, 1996, 2001b; SILVA et al., 1999; NISHIWAKI et al., 1998; LARSEN; SIGMUND; BOUWSTRA, 1997).

Em vista disso é fundamental representar na forma discreta as equações governantes dos microatuadores eletrotermomecânicos. Esta discretização é feita utilizando os conceitos dos elementos finitos. O domínio de projeto fixo é assumido bidimensional e discretizado utilizando elementos finitos retangulares de quatro nós (ver seção 2.2.1). O número de elementos usados na discretização é $N$ e o número de nós é $n$. A estrutura é modelada usando a hipótese de estado plano de tensão ou "plane stress" (COOK; YOUNG, 1985).

Como o objetivo da OT baseada no método das densidades é otimizar a distribuição do material através do controle da densidade em cada elemento finito, determina-se que as propriedades do material dependam do valor da pseudodensidade (variáveis de projeto) $\rho^{e}$ do elemento $e$. Essas propriedades são: cada 
componente da matriz de elasticidade $\boldsymbol{D}$ (calculada a partir das propriedades de cada material), coeficiente de expansão térmica $\alpha$, condutividade elétrica $\sigma_{0}$ ou condutividade térmica $\sigma_{1}$. O vetor que contém as variáveis de projeto de todo o domínio é definido como $\boldsymbol{\rho}$, de dimensão $N$, usando-se o modelo de material de função de pico (ver seção 4.2). Para simplificar, nas equações (2.1) a (2.3) assume-se que as forças de corpo e as fontes internas de corrente elétrica são nulas, assim, o sistema eletrotermomecânico discretizado pode ser modelado por três subproblemas de elementos finitos:

- Problema elétrico

$$
K_{0}(\rho) U_{0}(\rho)=P_{0}(\rho)
$$

- Problema térmico

$$
K_{1}(\rho) U_{1}(\rho)=P_{1}\left(U_{0}(\rho), \rho\right)
$$

- Domínio elástico

$$
K_{2}(\rho) U_{2}(\rho)=P_{2}\left(U_{1}(\rho), \rho\right)
$$

Como no caso das equações governantes da seção 2.1 , o índice 0 se refere à modelagem elétrica, 1, à térmica, e 2, à mecânica. Conforme já discutido, o campo elétrico resulta em aquecimento térmico, o qual resulta em deformação térmica. Portanto, os problemas de elemento finito (2.4) até (2.6) devem ser solucionados na ordem em que eles estão apresentados. Para os problemas elétrico e térmico, $\boldsymbol{K}_{\mathbf{0}}$ e $\boldsymbol{K}_{1}$ representam a condutividade global elétrica e térmica, respectivamente, e são matrizes $(n \times n)$, já que existe somente um grau de liberdade elétrico e térmico por nó. $\boldsymbol{K}_{2}$ é a matriz de rigidez global de dimensões $(2 n \times 2 n)$ pois, neste caso, existem dois graus de liberdade de deslocamentos por nó (problema bidimensional). $\boldsymbol{U}_{0}$ e $\boldsymbol{U}_{1}$ são os vetores de tensão elétrica e variação de temperatura em relação ao estado livre de tensões térmicas, ambos de dimensão $n . \boldsymbol{U}_{\mathbf{2}}$ é o vetor de deslocamentos nodais de dimensão $2 n . \boldsymbol{P}_{\mathbf{0}}$ e $\boldsymbol{P}_{\mathbf{1}}$ são os vetores, de dimensão $n$, de carga elétrica e térmica nodal. $\boldsymbol{P}_{\mathbf{2}}$ é o vetor, de dimensão $2 n$, de carga elástica nodal. Todas as matrizes e vetores são dependentes do vetor $\boldsymbol{\rho}$ de pseudo-densidades dos elementos.

O sistema de matrizes globais é montado de modo usual (através do operador de montagem $M$ ) como uma soma de todas as matrizes dos elementos, seguindo a conectividade entre os graus de liberdade local e global (BATHE, 1995; 
ZIENKIEWICZ; TAYLOR, 1991; SIGMUND, 2001b):

$$
\begin{aligned}
& \boldsymbol{K}_{\mathbf{0}}(\boldsymbol{\rho})=\stackrel{N}{M_{e=1}^{N}} \boldsymbol{K}_{\mathbf{0}}^{\boldsymbol{e}}\left(\rho^{e}\right) \\
& \boldsymbol{K}_{\mathbf{1}}(\boldsymbol{\rho})=\stackrel{N}{e=1}_{e=1}^{N}\left(\boldsymbol{K}_{\mathbf{1}}^{\boldsymbol{e}}\left(\rho^{e}\right)+\boldsymbol{h}^{\boldsymbol{e}}\right) \\
& \boldsymbol{K}_{\mathbf{2}}(\boldsymbol{\rho})=\stackrel{N}{M} \boldsymbol{K}_{\mathbf{2}}^{\boldsymbol{e}}\left(\rho^{e}\right)
\end{aligned}
$$

onde $\boldsymbol{K}_{\mathbf{0}}^{e}$ é a matriz de condutividade elétrica de dimensão $\left(n_{e} \times n_{e}\right) ; \boldsymbol{K}_{\mathbf{1}}^{e}$ e $\boldsymbol{h}^{e}$ são as matrizes de condutividade térmica e convecção dos elementos, respectivamente, de dimensões $\left(n_{e} \times n_{e}\right)$; e $\boldsymbol{K}_{\mathbf{2}}^{e}$ é a matriz de rigidez do elemento de dimensão $\left(2 n_{e} \times 2 n_{e}\right) \cdot n_{e}$ é o número de nós por elemento.

A matriz global $\boldsymbol{K}_{1}$ é obtida pela soma de $\boldsymbol{h}^{e}$ a $\boldsymbol{K}_{1}^{e}$ devido ao termo $h\left(\bar{u}_{1 \infty}-\right.$ $u_{1}$ ) da carga térmica $b_{1}$ (equação $2.2 \mathrm{~b}$ ), lembrando-se que $\boldsymbol{U}_{1}$ representa a variação de temperatura nodal em relação ao estado livre de tensões térmicas.

Da mesma forma, são montados no sistema global como uma somatória dos carregamentos por elemento, o vetor de carga do problema eletrotérmico $\left(\boldsymbol{P}_{1}\right)$ e do problema termoelástico $\left(\boldsymbol{P}_{\mathbf{2}}\right)$.

A continuação do estudo mantém o foco no elemento, já que é neste nível onde se apresentam as diferenças na formulação pelo MEF das equações constitutivas dos microatuadores eletrotermomecânicos. Primeiro, é formulada a expressão para o problema termoelástico, especificamente para a matriz de rigidez do elemento e o vetor de carga, sendo apresentados cada um dos seus componentes. Após a formulação do problema termoelástico, os problemas térmico e eletrotérmico são descritos.

\subsubsection{MEF no problema termoelástico}

\section{Matriz de rigidez do elemento retangular isoparamétrico}

No problema termoelástico, trabalha-se com um elemento retangular de quatro nós com dois graus de liberdade por nó, o deslocamento no eixo $x$ (deslocamento horizontal) e no eixo $y$ (deslocamento vertical). O elemento retangular com os graus de liberdade de deslocamento é apresentado na figura 2.2(a) nas suas coordenadas naturais e, na figura 2.2(b), nas suas coordenadas físicas, entendendo que o sistema de coordenadas naturais $(\xi, \eta)$ é o sistema padrão ou local, enquanto que o sistema "real" é denominado sistema de coordenadas físicas ou global $(x, y)$ (BATHE, 1995; ZIENKIEWICZ; TAYLOR, 1991; COOK; MALKUS; PLESHA, 1989): 


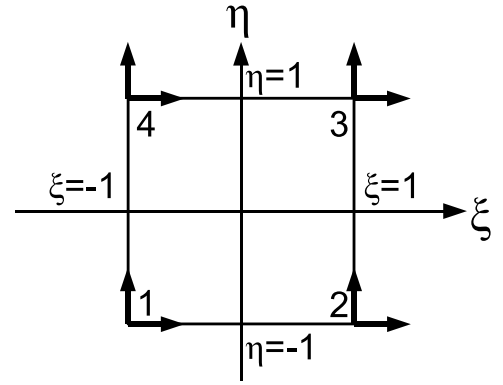

(a) sistema de coordenadas local (com deslocamentos dos nós)

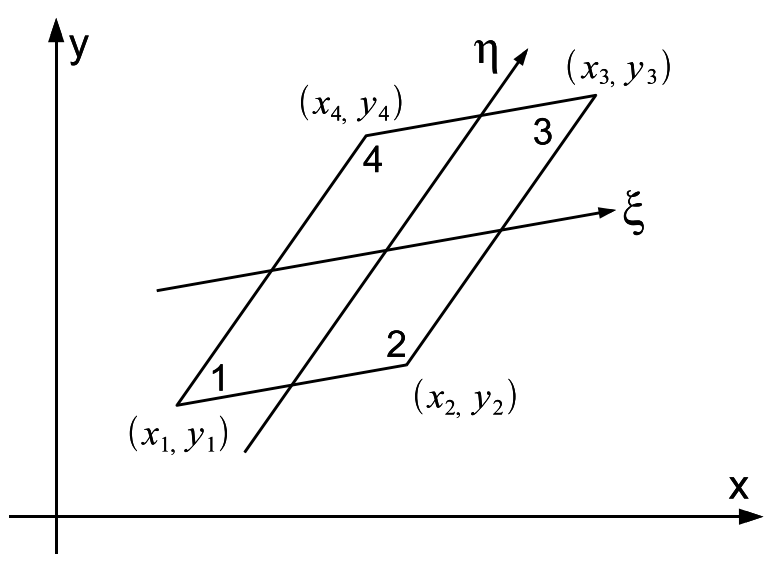

(b) sistema de coordenadas global

Figura 2.2: Elemento retangular de quatro nós.

Todo cálculo por elementos finitos, sendo um método aproximado, busca uma expressão aproximada da solução, em outras palavras, busca aproximar os deslocamentos $\boldsymbol{v}^{e}$ de qualquer ponto do elemento mediante um vetor coluna $\hat{\boldsymbol{v}}^{e}(x, y)$ (ZIENKIEWICZ; TAYLOR, 1991):

$$
\boldsymbol{v}^{\boldsymbol{e}}(x, y) \approx \hat{\boldsymbol{v}}^{\boldsymbol{e}}=\sum_{i=1}^{4} N_{i} \boldsymbol{U}_{\mathbf{2} \boldsymbol{x} \boldsymbol{y} \boldsymbol{i}}^{\boldsymbol{e}}=\boldsymbol{N}_{\boldsymbol{f}} \boldsymbol{U}_{\mathbf{2}}^{\boldsymbol{e}}
$$

onde $N_{i}$ é a função de forma relativa ao nó $i, \boldsymbol{U}_{\mathbf{2} x y \boldsymbol{i}}^{e}$ é o vetor de deslocamento do nó $i$ (dimensão 2 - em $x$ e $y$ ), $\boldsymbol{U}_{2}^{e}$ é o vetor dos deslocamentos nodais do elemento $e$ de dimensão $2 n_{e}$, e $\boldsymbol{N}_{\boldsymbol{f}}$ é o vetor das funções de forma expressas em função de variáveis independentes, como as coordenadas locais $-1 \leq \xi \leq 1$ e $-1 \leq \eta \leq 1$ (ver figura 2.2(a)). A matriz das funções de forma para o elemento isoparamétrico apresentado na figura 2.2(a) é composta pelos seguintes polinômios (BATHE, 1995; ZIENKIEWICZ; TAYLOR, 1991; COOK; MALKUS; PLESHA, 1989):

$$
\begin{aligned}
& N_{1}(\xi, \eta)=\frac{1}{4}(1-\xi)(1-\eta) \\
& N_{2}(\xi, \eta)=\frac{1}{4}(1+\xi)(1-\eta) \\
& N_{3}(\xi, \eta)=\frac{1}{4}(1+\xi)(1+\eta) \\
& N_{4}(\xi, \eta)=\frac{1}{4}(1-\xi)(1+\eta)
\end{aligned}
$$

Conhecendo as funções de forma, também é possível mapear um ponto $(\xi, \eta)$ no elemento retangular isoparamétrico a um ponto $(x, y)$ do elemento em coordenadas globais, possivelmente com distorção como se observa na figura 
2.2(b) (BATHE, 1995):

$$
\begin{aligned}
& x=\sum_{i=1}^{4} N_{i}(\xi, \eta) x_{i} \\
& y=\sum_{i=1}^{4} N_{i}(\xi, \eta) y_{i}
\end{aligned}
$$

As equações (2.10) e (2.12) caracterizam a formulação dos elementos finitos paramétricos, nos quais na interpolação das coordenadas dos elementos e dos deslocamentos são usadas as mesmas funções de interpolação definidas no sistema de coordenadas naturais. Assim, uma vez conhecidos os deslocamentos para todos os pontos do elemento são determinadas as deformações em qualquer ponto do mesmo. Estas resultam numa relação que são escritas na forma matricial (ZIENKIEWICZ; TAYLOR, 1991):

$$
\varepsilon^{e}=\boldsymbol{L} v^{e}
$$

onde $\boldsymbol{L}$ é um operador linear apropriado. Considerando a equação (2.10), a expressão anterior pode ser escrita como:

$$
\hat{\varepsilon}^{e}=B_{2} U_{2}^{e}
$$

onde:

$$
B_{2}=L N_{f}
$$

No caso particular de estado plano de tensão, as deformações consideradas são as que ocorrem no plano e são expressas em função dos deslocamentos, através das relações que definem o operador $\boldsymbol{L}$ (ZIENKIEWICZ; TAYLOR, 1991):

$$
\boldsymbol{\varepsilon}^{\boldsymbol{e}}=\left\{\begin{array}{c}
\varepsilon_{x} \\
\varepsilon_{y} \\
\gamma_{x y}
\end{array}\right\}=\left\{\begin{array}{c}
\frac{\partial u_{2(x)}}{\partial x} \\
\frac{\partial u_{2(y)}}{\partial y} \\
\frac{\partial u_{2(x)}}{\partial y}+\frac{\partial u_{2(y)}}{\partial x}
\end{array}\right\}=\left[\begin{array}{cc}
\frac{\partial}{\partial x} & 0 \\
0 & \frac{\partial}{\partial y} \\
\frac{\partial}{\partial y} & \frac{\partial}{\partial x}
\end{array}\right]\left\{\begin{array}{l}
u_{2(x)} \\
u_{2(y)}
\end{array}\right\}=\boldsymbol{L} \boldsymbol{v}^{\boldsymbol{e}}
$$

Portanto, da equação (2.15) deduz-se que a matriz $\boldsymbol{B}_{2}$ é uma matriz de operadores diferenciais das funções de forma $N_{i}$ que, para o elemento retangular de quatro nós em tensão plana, é dada por:

$$
\boldsymbol{B}_{\mathbf{2 i}}=\left[\begin{array}{cc}
\frac{\partial N_{i}}{\partial x} & 0 \\
0 & \frac{\partial N_{i}}{\partial y} \\
\frac{\partial N_{i}}{\partial y} & \frac{\partial N_{i}}{\partial x}
\end{array}\right]_{i=1 \rightarrow 4}
$$


Nota-se que é necessária uma transformação para avaliar as matrizes $\boldsymbol{B}_{\mathbf{2} i}$, já que $N_{i}$ é definida em função de coordenadas locais. Portanto, é necessário um procedimento que expresse as derivadas globais do tipo que apareceram na equação (2.17) em função das derivadas locais. Isto é conseguido através da matriz jacobiana $\boldsymbol{J}$ que, no caso específico de estruturas bidimensionais (onde a dimensão da coordenada $z$ é desconsiderada), expressa-se como (ZIENKIEWICZ; TAYLOR, 1991):

$$
\left\{\begin{array}{c}
\frac{\partial N_{i}}{\partial x} \\
\frac{\partial N_{i}}{\partial y}
\end{array}\right\}=\boldsymbol{J}^{-1}\left\{\begin{array}{c}
\frac{\partial N_{i}}{\partial \xi} \\
\frac{\partial N_{i}}{\partial \eta}
\end{array}\right\}
$$

onde:

$$
\boldsymbol{J}=\left[\begin{array}{ll}
\frac{\partial x}{\partial \xi} & \frac{\partial y}{\partial \xi} \\
\frac{\partial x}{\partial \eta} & \frac{\partial y}{\partial \eta}
\end{array}\right]
$$

Com base nas expressões anteriormente desenvolvidas e segundo a teoria do $\mathrm{MEF}$, a matriz de rigidez do elemento pode ser encontrada mediante o princípio de conservação de energia (BATHE, 1995; ZIENKIEWICZ; TAYLOR, 1991). Assim, a energia potencial de deformação do elemento $(U)$ deve ser igual à energia potencial das cargas externas ou, em outras palavras, ao trabalho realizado pelas tensões externas nos nós do elemento $(W)$. Considerando o caso bidimensional:

$$
U=W
$$

com:

$$
\begin{gathered}
U=\frac{1}{2} \int_{A^{e}} \boldsymbol{\sigma}^{e t} \varepsilon^{e} d A^{e}=\frac{1}{2} \int_{A^{e}} \boldsymbol{\varepsilon}^{e t} \boldsymbol{\sigma}^{\boldsymbol{e}} d A^{e} \\
W=\frac{1}{2} \boldsymbol{P}_{\mathbf{2}}^{\boldsymbol{e t}} \boldsymbol{U}_{\mathbf{2}}^{\boldsymbol{e}}=\frac{1}{2} \boldsymbol{U}_{\mathbf{2}}^{\boldsymbol{e t}} \boldsymbol{P}_{\mathbf{2}}^{\boldsymbol{e}}=\frac{1}{2} \boldsymbol{P}_{\mathbf{2}}^{\boldsymbol{e} t} \boldsymbol{K}_{\mathbf{2}}^{\boldsymbol{e}} \boldsymbol{U}_{\mathbf{2}}^{\boldsymbol{e}}
\end{gathered}
$$

Igualando $W$ a $U$ :

$$
\frac{1}{2} \boldsymbol{U}_{\mathbf{2}}^{e t} \boldsymbol{K}_{\mathbf{2}}^{e} \boldsymbol{U}_{\mathbf{2}}^{e}=\frac{1}{2} \int_{A^{e}} \boldsymbol{\varepsilon}^{e t} \boldsymbol{\sigma}^{e} d A^{e}
$$

Sabendo que a relação entre tensões e deformações tem a forma: $\sigma^{e}=\boldsymbol{D} \varepsilon^{e}$ e substituindo a equação (2.14) na equação (2.23), obtém-se:

$$
\boldsymbol{U}_{\mathbf{2}}^{e t} \boldsymbol{K}_{\mathbf{2}}^{e} \boldsymbol{U}_{\mathbf{2}}^{\boldsymbol{e}}=\int_{A^{e}} \boldsymbol{U}_{\mathbf{2}}^{e t} \boldsymbol{B}_{\mathbf{2}}{ }^{t} \boldsymbol{D} \boldsymbol{B}_{\mathbf{2}} \boldsymbol{U}_{\mathbf{2}}^{e} d A^{e}=\boldsymbol{U}_{\mathbf{2}}{ }^{e t}\left(\int_{A^{e}} \boldsymbol{B}_{\mathbf{2}}{ }^{t} \boldsymbol{D} \boldsymbol{B}_{\mathbf{2}} d A^{e}\right) \boldsymbol{U}_{\mathbf{2}}^{\boldsymbol{e}}
$$

Portanto a matriz de rigidez do elemento retangular isoparamétrico é:

$$
\boldsymbol{K}_{\mathbf{2}}^{e}=\int_{A^{e}} \boldsymbol{B}_{\mathbf{2}}{ }^{t} \boldsymbol{D} \boldsymbol{B}_{\mathbf{2}} d A^{e}
$$


onde a matriz de elasticidade $\boldsymbol{D}$ é a matriz cujos componentes são interpolados um a um segundo o modelo de material discutido na seção 4.2 entre os componentes correspondentes das diversas $\boldsymbol{D}_{\boldsymbol{m}}$, uma para cada material $m$. Para o estado plano de tensões, as $\boldsymbol{D}_{\boldsymbol{m}}$ são dadas por:

$$
\boldsymbol{D}_{\boldsymbol{m}}=\frac{E_{m}}{1-\nu_{m}^{2}}\left[\begin{array}{ccc}
1 & \nu_{m} & 0 \\
\nu_{m} & 1 & 0 \\
0 & 0 & \frac{1-\nu_{m}}{2}
\end{array}\right]
$$

onde $E_{m}$ é o módulo de elasticidade e $\nu_{m}$ é o coeficiente de Poisson, ambos relacionados a um material $m$.

Por outro lado, o elemento de volume sobre o qual é efetuada a integração tem que ser escrito em função das coordenadas locais (BATHE, 1995; ZIENKIEWICZ; TAYLOR, 1991). Assim, é necessário mudar convenientemente os limites de integração na equação (2.25):

$$
d A^{e}=d x d y=\operatorname{det} \boldsymbol{J} d \xi d \eta
$$

Explicitando a dependência da matriz de rigidez em relação à densidade relativa $\rho^{e}$, a matriz de rigidez fica:

$$
\boldsymbol{K}_{\mathbf{2}}^{\boldsymbol{e}}(\boldsymbol{\rho})=\int_{A^{e}} \boldsymbol{B}_{\mathbf{2}}{ }^{t} \boldsymbol{D}\left(\rho^{e}\right) \boldsymbol{B}_{\mathbf{2}} \operatorname{det} \boldsymbol{J} d \xi d \eta
$$

\section{Vetor de carga do elemento no problema termoelástico}

No caso de problemas elásticos gerais tridimensionais, o vetor de carga pode estar formado por qualquer uma das seguintes componentes (BATHE, 1995):

$$
P_{2}=P_{2 B}+P_{2 S}-P_{2 I}+P_{2 C}
$$

onde $\boldsymbol{P}_{2 B}$ é o vetor de carga global que inclui os efeitos de forças de volume; $\boldsymbol{P}_{2 S}$ inclui o efeito das forças de superfície; $\boldsymbol{P}_{2 \boldsymbol{I}}$ inclui o efeito global das tensões iniciais; e o efeito das cargas nodais concentradas é representado pelo vetor $\boldsymbol{P}_{\mathbf{2}}$. Todos estes vetores têm dimensão $2 n$, lembrando que $n$ é o número de nós da estrutura discretizada.

O vetor $\boldsymbol{P}_{\mathbf{2}}$ (BATHE, 1995) pode ser expresso, para o caso bidimensional, como:

$$
\boldsymbol{P}_{\mathbf{2 B}}=\stackrel{N}{M} \int_{e=1} \boldsymbol{N}_{A^{e}}^{t} \boldsymbol{f}^{\boldsymbol{B}} d A^{e}
$$


onde $f^{B}$ são as forças de volume aplicadas externamente ao elemento, $\boldsymbol{N}_{\boldsymbol{f}}$ é o vetor das funções de forma anteriormente definidas nas equações (2.11), dA está definida pela equação (2.27).

Da mesma forma, o vetor $\boldsymbol{P}_{\mathbf{2}}$ (BATHE, 1995) pode ser expresso como:

$$
\boldsymbol{P}_{\mathbf{2} \boldsymbol{S}}=\stackrel{N}{M} \int_{e=1} \int_{L_{1}^{e}, \ldots, L_{q}^{e}} \boldsymbol{N}_{\boldsymbol{f}}^{t} \boldsymbol{f}^{\boldsymbol{S}} d L^{e}
$$

onde $f^{S}$ são as forças de superfície aplicadas externamente ao elemento, em seu contorno; $\boldsymbol{N}_{\boldsymbol{f}}$ é o vetor das funções de forma; e $q$, o número de áreas ou superfícies do elemento (no caso bidimensional, os contornos do elemento).

O vetor $\boldsymbol{P}_{2 \boldsymbol{I}}$ (BATHE, 1995) está definido como:

$$
\boldsymbol{P}_{\mathbf{2 I}}=\stackrel{N}{M} \int_{e=1} \boldsymbol{B}_{A^{e}}{ }^{t} \boldsymbol{\tau}_{\boldsymbol{I}}^{e} d A^{e}
$$

onde $\boldsymbol{\tau}_{\boldsymbol{I}}^{e}$ são as tensões iniciais do elemento $e$ e $\boldsymbol{B}_{2}$, a matriz de operadores diferenciais das funções de forma $N_{i}$, definida na equação (2.17).

No problema termoelástico do projeto de microatuadores eletrotermomecânicos, o único efeito importante no vetor de carga $\boldsymbol{P}_{2}$ é o das tensões térmicas iniciais geradas pela variação de temperatura entre os diversos nós do elemento e do micromecanismo em geral, lembrando que essas variações de temperatura nodais estão contidas no vetor $\boldsymbol{U}_{\mathbf{1}}$ para o micromecanismo (vetor global da estrutura) e no vetor $\boldsymbol{U}_{1}^{e}$ para cada elemento $e$ (vetor de temperatura local) que são resultantes do problema eletrotérmico. Assim, assumindo-se o subproblema termoelástico isolado dos outros dois, essas tensões térmicas iniciais seriam interpretadas como tensões residuais, representadas na equação (2.32) como $\tau_{\boldsymbol{I}}^{e}$. Podem também existir cargas nodais concentradas $\boldsymbol{P}_{\mathbf{2 C}}$ em nós onde há condições de contorno elásticas, como engastamentos do mecanismo. Como não se pretende aplicar explicitamente forças nos nós, o vetor $\boldsymbol{P}_{2 C}$ simboliza as reações sobre o mecanismo devido às condições de contorno elásticas. O vetor de carga do problema termoelástico poderia ser expresso como (ANANTHASURESH, 2003; MANKAME; ANANTHASURESH, 2001; SIGMUND, 2001b; BATHE, 1995):

$$
\begin{gathered}
\boldsymbol{P}_{\mathbf{2}}\left(\boldsymbol{U}_{\mathbf{1}}, \boldsymbol{\rho}\right)=-\boldsymbol{P}_{\mathbf{2 I}}+\boldsymbol{P}_{\mathbf{2 C}}=-\stackrel{N}{M_{e=1}^{N}} \boldsymbol{P}_{\mathbf{2} \boldsymbol{I}}^{\boldsymbol{e}}\left(\boldsymbol{U}_{\mathbf{1}}^{\boldsymbol{e}}, \rho^{e}\right)+\boldsymbol{P}_{\mathbf{2 C}}(\boldsymbol{\rho}) \\
\boldsymbol{P}_{\mathbf{2} \boldsymbol{I}}^{\boldsymbol{e}}\left(\boldsymbol{U}_{\mathbf{1}}^{\boldsymbol{e}}, \rho^{e}\right)=\int_{A^{e}} \boldsymbol{B}_{\mathbf{2}}{ }^{t} \boldsymbol{\tau}_{\boldsymbol{I}}^{e}\left(\boldsymbol{U}_{\mathbf{1}}^{e}, \rho^{e}\right) d A^{e} \\
\boldsymbol{P}_{\mathbf{2}}\left(\boldsymbol{U}_{\mathbf{1}}, \boldsymbol{\rho}\right)=-\stackrel{N}{M} \int_{A^{e}} \boldsymbol{B}_{\mathbf{2}}{ }^{t} \boldsymbol{\tau}_{\boldsymbol{I}}^{e}\left(\boldsymbol{U}_{\mathbf{1}}^{\boldsymbol{e}}, \rho^{e}\right) d A^{e}+\boldsymbol{P}_{\mathbf{2} \boldsymbol{C}}(\boldsymbol{\rho})
\end{gathered}
$$


onde $\boldsymbol{P}_{2 I}^{e}$ é o vetor de tensões iniciais da carga elástica do elemento $e$.

Neste caso, sabe-se que a tensão total $\boldsymbol{\tau}$ para um elemento sujeito a uma dada distribuição de temperatura nodal, devido à deformação total $\varepsilon$ e às deformações térmicas $\varepsilon_{t}$, é (BATHE, 1995):

$$
\tau=D\left(\varepsilon-\varepsilon_{t}\right)
$$

onde, sob estado plano de tensão:

$$
\varepsilon_{t}=\left\{\begin{array}{c}
\varepsilon_{t x} \\
\varepsilon_{t y} \\
\gamma_{t x y}
\end{array}\right\}=\alpha\left(\theta-\theta_{0}\right)\left\{\begin{array}{l}
1 \\
1 \\
0
\end{array}\right\}
$$

onde $\alpha$ é o coeficiente de expansão térmica, $\theta$ é a temperatura num ponto do elemento e $\theta_{0}$, a temperatura no estado livre de tensões térmicas, nesse trabalho considerada também como a temperatura ambiente.

Se os deslocamentos nodais são nulos, $\varepsilon=0$, as tensões devido às deformações térmicas podem ser propostas como tensões iniciais. Portanto, substituindo a equação (2.37) em (2.36) e considerando o modelo de material, obtém-se as tensões iniciais do elemento (BATHE, 1995):

$$
\begin{aligned}
& \boldsymbol{\tau}_{\boldsymbol{I}}^{e}\left(\boldsymbol{U}_{1}^{e}, \rho^{e}\right)=\boldsymbol{D}\left(\rho^{e}\right)\left(-\alpha\left(\rho^{e}\right)\left(\theta-\theta_{0}\right)\left\{\begin{array}{l}
1 \\
1 \\
0
\end{array}\right\}\right)= \\
& =\boldsymbol{D}\left(\rho^{e}\right)\left[-\alpha\left(\rho^{e}\right)\left(\sum_{i=1}^{4} N_{i} U_{1 i}^{e}\right)\left\{\begin{array}{l}
1 \\
1 \\
0
\end{array}\right\}\right] \\
& \boldsymbol{\tau}_{\boldsymbol{I}}^{\boldsymbol{e}}\left(\boldsymbol{U}_{\mathbf{1}}^{\boldsymbol{e}}, \rho^{e}\right)=-\alpha\left(\rho^{e}\right) \boldsymbol{D}\left(\rho^{e}\right)\left\{\begin{array}{l}
1 \\
1 \\
0
\end{array}\right\}\left(\sum_{i=1}^{4} N_{i} U_{1 i}^{e}\right) \\
& \boldsymbol{\tau}_{\boldsymbol{I}}^{e}\left(\boldsymbol{U}_{\mathbf{1}}^{\boldsymbol{e}}, \rho^{e}\right)=-\alpha\left(\rho^{e}\right) \boldsymbol{D}\left(\rho^{e}\right)\left\{\begin{array}{l}
1 \\
1 \\
0
\end{array}\right\} \boldsymbol{N}_{\boldsymbol{f}} \boldsymbol{U}_{\mathbf{1}}^{\boldsymbol{e}}
\end{aligned}
$$

onde $U_{1 i}^{e}$ ( $i$ de 1 até 4$)$ representa a variação de temperatura para cada um dos nós do elemento retangular, resultante do problema eletrotérmico.

Existindo condições de contorno de deslocamento, estas serão de Dirichlet 


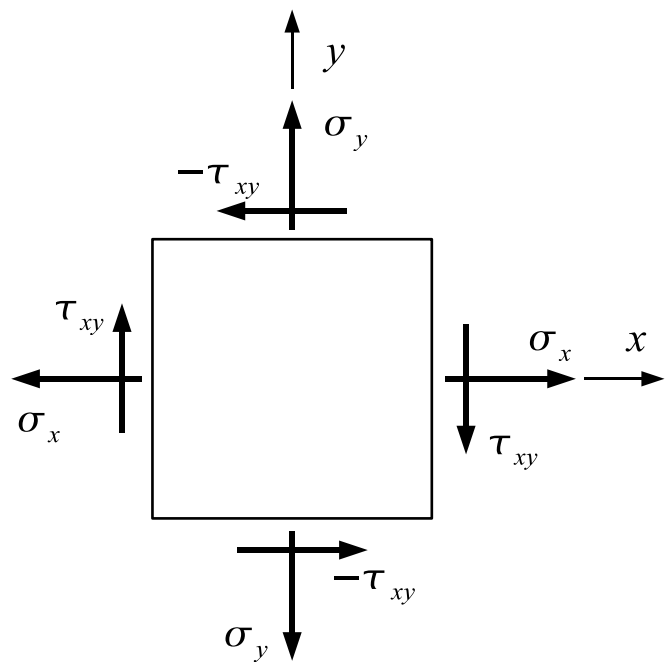

Figura 2.3: Tensões normais e de cisalhamento em $x$ e $y$.

(apêndice A) e as reações aparecerão no vetor $\boldsymbol{P}_{2 C}$ nas posições relativas aos graus de liberdade impostos, tendo valores nulos nas demais. Nos casos estudados, essas reações não são de interesse e não serão calculadas, já que se deseja apenas os deslocamentos nodais $\boldsymbol{U}_{2}$.

\section{Pós-processamento}

Uma vez solucionado o problema termoelástico para o estado plano de tensões pelo MEF, é possível calcular as tensões mecânicas, conforme mostrado pela equação (2.36). Considerando um ponto no interior de um dado elemento $e$, os deslocamentos nodais $\boldsymbol{U}_{2}^{e}$ e as variações de temperatura nodais $\boldsymbol{U}_{1}^{e}$, as tensões mecânicas são dadas por:

$$
\tau^{e}=\left\{\begin{array}{c}
\tau_{x x} \\
\tau_{y y} \\
\tau_{x y}
\end{array}\right\}=\boldsymbol{D}\left(\hat{\varepsilon}^{e}-\hat{\varepsilon}_{t}^{e}\right)
$$

onde $\tau_{x x}, \tau_{y y}$ e $\tau_{x y}$ são as tensões normais e de cisalhamento em $x$ e $y$; $\hat{\varepsilon}^{e}$ é a deformação total aproximada num dado ponto do elemento $e$, dada pela equação (2.14); $\hat{\varepsilon}_{t}^{e}$ é a deformação térmica aproximada num dado ponto do elemento $e$, dada por:

$$
\hat{\varepsilon}_{t}^{e}=\alpha\left\{\begin{array}{l}
1 \\
1 \\
0
\end{array}\right\} \boldsymbol{N}_{\boldsymbol{f}} \boldsymbol{U}_{\mathbf{1}}^{\boldsymbol{e}}
$$

A figura 2.3 mostra as tensões normais e de cisalhamento numa parte arbitrária do domínio. 
A partir das tensões, pode-se calcular as duas tensões principais não nulas (SHIGLEY; MISCHKE, 1989):

$$
\begin{aligned}
\sigma_{A} & =\frac{\tau_{x x}+\tau_{y y}}{2}+\sqrt{\left(\frac{\tau_{x x}-\tau_{y y}}{2}\right)^{2}+\tau_{x y}^{2}} \\
\sigma_{B} & =\frac{\tau_{x x}+\tau_{y y}}{2}-\sqrt{\left(\frac{\tau_{x x}-\tau_{y y}}{2}\right)^{2}+\tau_{x y}^{2}}
\end{aligned}
$$

A tensão de von Mises, dada pelas tensões principais $\sigma_{A}$ e $\sigma_{B}$, é calculada por:

$$
\sigma_{v M}=\sqrt{\sigma_{A}^{2}-\sigma_{A} \sigma_{B}+\sigma_{B}^{2}}
$$

\subsubsection{MEF no problema elétrico e eletrotérmico}

\section{Matriz de condutividade elétrica e térmica do elemento retangular isoparamétrico}

No problema elétrico e eletrotérmico, trabalha-se com um elemento retangular de quatro nós com um grau de liberdade por nó, que são a tensão elétrica e a variação de temperatura, respectivamente. As matrizes de condutividade elétrica, térmica e de convecção são determinadas mediante o princípio de conservação de energia de forma equivalente ao procedimento expresso da equação (2.20) até (2.25) para a matriz de rigidez, sendo respectivamente dadas por Sigmund (2001b), Bathe (1995), Cook, Malkus e Plesha (1989):

$$
\begin{aligned}
\boldsymbol{K}_{\mathbf{0}}^{\boldsymbol{e}}\left(\rho^{e}\right) & =\sigma_{0}\left(\rho^{e}\right) \int_{A^{e}} \boldsymbol{B}_{\mathbf{0}}{ }^{t} \boldsymbol{B}_{\mathbf{0}} d A^{e} \\
\boldsymbol{K}_{\mathbf{1}}^{\boldsymbol{e}}\left(\rho^{e}\right) & =\sigma_{1}\left(\rho^{e}\right) \int_{A^{e}} \boldsymbol{B}_{\mathbf{1}}{ }^{t} \boldsymbol{B}_{\mathbf{1}} d A^{e} \\
\boldsymbol{h}^{e} & =h_{0} \int_{S^{e}} \boldsymbol{N}_{\boldsymbol{f}}^{t} \boldsymbol{N}_{\boldsymbol{f}} d S^{e}
\end{aligned}
$$

onde $\sigma_{0}\left(\rho^{e}\right)$ e $\sigma_{1}\left(\rho^{e}\right)$ são os coeficientes de condutividade elétrica e térmica do elemento $e$ de acordo com o modelo de material, respectivamente (ver seção 4.2). $\boldsymbol{N}_{\boldsymbol{f}}$ é o vetor das funções de forma, cujas componentes $N_{i}$ são definidas nas equações (2.11). O coeficiente de convecção $h_{0}$ é assumido constante, sem variar com a densidade relativa do elemento, caso contrário, elementos de baixa densidade não seriam resfriados (SIGMUND, 2001b). $\boldsymbol{B}_{\mathbf{0}}$ e $\boldsymbol{B}_{\mathbf{1}}$ são as matrizes de 
gradiente elétrico e de gradiente térmico, respectivamente, definidas como:

$$
\boldsymbol{B}_{\mathbf{0} \boldsymbol{i}}=\boldsymbol{B}_{\mathbf{1} \boldsymbol{i}}=\left[\begin{array}{l}
\frac{\partial N_{i}}{\partial x} \\
\frac{\partial N_{i}}{\partial y}
\end{array}\right]_{i=1 \rightarrow 4}
$$

\section{Vetor de carga do elemento no problema eletrotérmico}

No caso de um problema térmico em geral, o vetor de carga $\boldsymbol{P}_{\mathbf{1}}$ é constituído de duas parcelas básicas (BATHE, 1995; COOK; MALKUS; PLESHA, 1989): o vetor de carga de fluxo de calor $\boldsymbol{P}_{\mathbf{1} \boldsymbol{F}}$ e a contribuição de carga devido às condições de fronteira de convecção $\boldsymbol{P}_{\mathbf{1} \text { conv }}$. O vetor de carga de fluxo de calor é formado pelo vetores de calor gerado por unidade de volume, $\boldsymbol{P}_{\mathbf{1 B}}$; de fluxo de calor sobre a superfície do corpo, $\boldsymbol{P}_{1 \boldsymbol{S}}$; e de fluxo de calor nodal concentrado, $\boldsymbol{P}_{\mathbf{1 C}}$. A expressão para cada um desses vetores é apresentada a seguir:

$$
P_{1}=P_{1 F}+P_{1 \text { conv }}
$$

- Carga relacionada com o fluxo de calor

$$
P_{1 F}=P_{1 B}+P_{1 S}+P_{1 C}
$$

onde (BATHE, 1995):

$$
\boldsymbol{P}_{\mathbf{1 B}}=\stackrel{N}{M} \int_{e=1} \boldsymbol{N}_{A^{e}}^{t} q^{B} d A^{e}
$$

onde $q^{B}$ é o termo que contém o fator gerador de calor interno por volume na estrutura como, por exemplo, o efeito Joule.

$$
\boldsymbol{P}_{\mathbf{1 S}}=\stackrel{N}{M} \int_{e=1} \boldsymbol{N}_{S^{e}}^{t} q^{S} d S^{e}
$$

onde $q^{S}$ é o termo que contém fluxo de calor pela superfície $S^{e}$.

- Carga relacionada com o efeito de convecção

$$
\boldsymbol{P}_{\mathbf{1} \text { conv }}=\stackrel{N}{M} \int_{e=1} h_{S_{c}^{e}} \boldsymbol{N}_{\boldsymbol{f}}^{t} \boldsymbol{N}_{\boldsymbol{f}} \boldsymbol{\theta}_{\text {conv }} d S^{e}
$$

onde $h_{0}$ é o coeficiente de convecção e $\boldsymbol{\theta}_{\text {conv }}$ é o vetor de variação de temperatura nodal do ambiente em relação ao estado livre de tensões térmicas. O subíndice $c$ refere-se à superfície do elemento onde existem condições de fronteira de convecção específicas. 
Neste trabalho, desconsideram-se os termos de fluxo de calor superficial $\boldsymbol{P}_{1 S}$. Uma parte do fluxo de calor nodal concentrado $\boldsymbol{P}_{1 C}$, além da parte explicitamente aplicada, pode aparecer devido à aplicação de condições de contorno de temperatura (tipo de Dirichlet, descrita no apêndice A) em alguns nós, mas não será calculada por não ser de interesse. $O$ fator predominante no projeto de micromecanismos eletrotermomecânicos é o calor gerado por unidade de volume devido ao efeito Joule (ANANTHASURESH, 2003; MANKAME; ANANTHASURESH, 2001; SIGMUND, 2001b). A carga relacionada ao efeito da convecção $\boldsymbol{P}_{1 \text { conv }}$ foi descartada pois a temperatura ambiente foi considerada igual à do estado livre de tensões térmicas e, portanto, $\boldsymbol{\theta}_{\text {conv }}$ é um vetor nulo. Desse modo, todo o efeito da convecção já foi considerado na montagem da matriz de condutividade térmica $\boldsymbol{K}_{\mathbf{1}}$ (equação (2.8)). Assim, o vetor de carga para o problema eletro-térmico, considerando a dependência das propriedades do material da densidade relativa, é:

$$
\begin{gathered}
\boldsymbol{P}_{\mathbf{1}}\left(U_{\mathbf{0}}, \boldsymbol{\rho}\right)=\boldsymbol{P}_{\mathbf{1 B}}+\boldsymbol{P}_{\mathbf{1} \boldsymbol{C}}=\stackrel{N}{M} \boldsymbol{P}_{\boldsymbol{e}=1}^{\boldsymbol{e}}\left(\boldsymbol{U}_{\mathbf{0}}^{\boldsymbol{e}}, \rho^{e}\right)+\boldsymbol{P}_{\mathbf{1} \boldsymbol{C}}(\boldsymbol{\rho}) \\
\boldsymbol{P}_{\mathbf{1 B}}^{\boldsymbol{e}}\left(\boldsymbol{U}_{\mathbf{0}}^{\boldsymbol{e}}, \rho^{e}\right)=\int_{A^{e}} \boldsymbol{N}_{\boldsymbol{f}}^{t} q^{B}\left(\boldsymbol{U}_{\mathbf{0}}^{\boldsymbol{e}}, \rho^{e}\right) d A^{e} \\
\boldsymbol{P}_{\mathbf{1}}\left(\boldsymbol{U}_{\mathbf{0}}, \boldsymbol{\rho}\right)=\stackrel{N}{M} \int_{e=1} \boldsymbol{N}_{A^{e}}^{t} q^{B}\left(\boldsymbol{U}_{\mathbf{0}}^{\boldsymbol{e}}, \rho^{e}\right) d A^{e}+\boldsymbol{P}_{\mathbf{1 C}}(\boldsymbol{\rho})
\end{gathered}
$$

onde $\boldsymbol{P}_{1 B}^{e}$ é o vetor de geração de calor interna da carga térmica do elemento $e$.

O efeito Joule é definido em forma matricial como (ANANTHASURESH, 2003; BATHE, 1995; ZIENKIEWICZ; TAYLOR, 1991):

$$
q^{B}\left(\boldsymbol{U}_{\mathbf{0}}^{\boldsymbol{e}}, \rho^{e}\right)=\boldsymbol{U}_{\mathbf{0}}^{\boldsymbol{e} t} \boldsymbol{B}_{\mathbf{0}}{ }^{t} \sigma_{0}\left(\rho^{e}\right) \boldsymbol{B}_{\mathbf{0}} \boldsymbol{U}_{\mathbf{0}}^{\boldsymbol{e}}
$$

onde $\boldsymbol{U}_{\mathbf{0}}^{e}$ é o vetor que contém as tensões elétricas nodais no elemento e $\boldsymbol{B}_{\mathbf{0}}$ é a matriz de gradiente elétrico.

A excitação do mecanismo eletrotermomecânico se inicia no domínio elétrico e pode ser por corrente elétrica nodal ou por tensão elétrica nodal prescrita. No caso de entrada por corrente elétrica prescrita, o vetor de carga $\boldsymbol{P}_{\mathbf{0}}$ corresponde a um vetor de dimensão $n$ com as correntes elétricas prescritas no microatuador, sendo independente de $\boldsymbol{\rho}$. No caso de entrada por tensão elétrica aplicada a alguns nós (tipo de Dirichlet, descrita no apêndice A), $\boldsymbol{P}_{\mathbf{0}}$ passa a ser dependente de $\boldsymbol{\rho}$, com termos não nulos apenas nos nós onde alguma tensão foi aplicada. Esses termos não serão calculados por não serem de interesse, bastando conhecer as tensões nodais $\boldsymbol{U}_{\mathbf{0}}$. 


\section{Pós-processamento}

Uma vez solucionado o problema elétrico, é possível calcular a densidade de corrente $\boldsymbol{j}$ num ponto qualquer no interior de um dado elemento $e$ a partir das tensões elétricas nodais $\boldsymbol{U}_{\mathbf{0}}^{e}$, dada por:

$$
\boldsymbol{j}=\left\{\begin{array}{l}
j_{x} \\
j_{y}
\end{array}\right\}=\sigma_{0}\left(-\boldsymbol{B}_{\mathbf{0}} \boldsymbol{U}_{\mathbf{0}}^{\boldsymbol{e}}\right)=-\sigma_{0} \boldsymbol{B}_{0} \boldsymbol{U}_{\mathbf{0}}^{\boldsymbol{e}}
$$

onde $\sigma_{0}\left(\rho^{e}\right)$ é a condutividade elétrica; $\boldsymbol{B}_{0}$ é a matriz de gradiente elétrico (equação (2.47). O produto $-\boldsymbol{B}_{0} \boldsymbol{U}_{\mathbf{0}}^{\boldsymbol{e}}$ corresponde ao campo elétrico do ponto sob análise.

O módulo da densidade de corrente pode ser obtido através das duas componentes, relativas aos eixos $x$ e $y$. A densidade de corrente de um certo ponto do MEMS eletrotermomecânico é determinante para o seu aquecimento. 


\section{O Método de Otimização Topológica (MOT)}

Neste capítulo, os tipos de otimização existentes serão apresentados como introdução para destacar a flexibilidade do MOT (seção 3.1), seguido dos conceitos básicos do MOT (seção 3.2). Na seção 3.3, um histórico do seu desenvolvimento é apresentado. Os problemas numéricos mais comuns observados nos resultados do MOT são descritos na seção 3.4, bem como maneiras de contorná-los.

\subsection{Introdução}

A geração sistemática de uma estrutura ótima de um certo domínio e que atenda aos requisitos exigidos, sejam eles de desempenho, tamanho físico ou custo, é interessante por permitir a obtenção de estruturas muito mais genéricas e otimizadas que as obtidas utilizando técnicas de tentativa e erro. Estas últimas técnicas fornecem resultados dependentes da experiência do projetista e em muito poucas ocasiões são as soluções ótimas do problema estrutural inicialmente proposto.

Podemos classificar os métodos de otimização estrutural em três categorias principais que se diferenciam principalmente pela definição dos parâmetros que mudam durante o processo de otimização: as variáveis de projeto. Essas categorias são: otimização paramétrica, otimização de forma e otimização topológica.

Otimização Paramétrica: uma certa geometria é pré-definida para a estrutura e são escolhidas como variáveis de projeto algumas medidas, ou razão entre medidas, que caracterizam tal geometria. Assim, neste caso, não é alterada a topologia inicial da estrutura, somente as suas dimensões $b, t$ e $h$ (ou a razão entre elas) do exemplo mostrado na figura 3.1.

Otimização de Forma: a otimização é realizada através de modificações das 


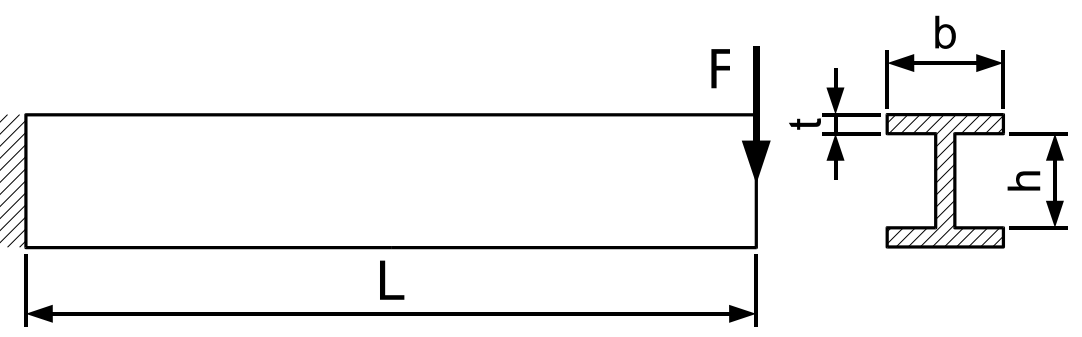

Figura 3.1: Otimização Paramétrica de uma viga em balanço (seção transversal à direita) - variáveis de projeto: $b, t$ e $h$ (parâmetros de uma geometria pré-definida).
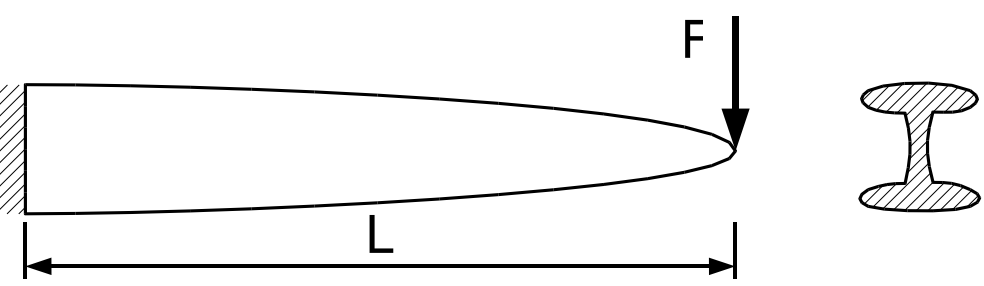

Figura 3.2: Otimização de Forma de uma viga em balanço (seção transversal à direita) - variáveis de projeto definem a geometria da estrutura (coordenadas das fronteiras ou coeficientes da curva que a representa).

fronteiras de uma geometria pré-definida. As variáveis de projeto podem ser as coordenadas de alguns pontos pertencentes ao contorno da peça ou parâmetros mais abstratos como coeficientes de uma curva que representa a forma da peça (por exemplo, curvas "spline"). Trata-se de uma abordagem mais genérica do que a anterior em que a forma externa da estrutura é otimizada, como pode ser visto na figura 3.2. Neste tipo de otimização, devido às formas complexas que podem ser obtidas, é utilizado geralmente o MEF para a análise das estruturas durante a otimização. A principal desvantagem da otimização de forma, nesse caso, é que a alteração da forma da estrutura distorce a malha de MEF, exigindo um remalhamento do domínio durante a otimização, o que não é fácil de implementar no problema tridimensional.

Otimização Topológica: a distribuição de material num domínio é livre, não estando vinculada a nenhuma limitação geométrica (a distribuição otimizada é posteriormente interpretada como uma geometria otimizada). Podem ser geradas topologias diferentes à inicialmente propostas (ponto de partida da otimização), permitindo-se inclusive a inclusão de elementos novos, como furos não especificados na geometria inicial (figura 3.3). Assim são obtidas altas reduções de massa e um grande aumento de desempenho em comparação à otimização de forma. Além disso, neste método não existe o problema de remalhamento do domínio de $\mathrm{MEF}$, como acontece na otimização de forma, pois trabalha-se sempre com um domínio fixo. 


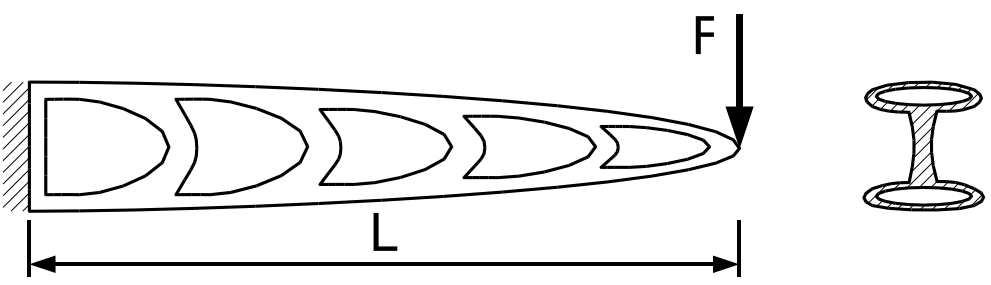

Figura 3.3: Otimização Topológica de uma viga em balanço (seção transversal à direita) - as variáveis de projeto definem a distribuição de material do domínio.

Na sua forma mais simples o MOT soluciona o problema da distribuição de uma dada quantidade de material num domínio de projeto fixo sujeito a determinadas cargas e condições de contorno, tal que uma certa função objetivo seja extremizada (maximizada ou minimizada). Desde a sua introdução computacional (BENDSØE; KIKUCHI, 1988), o método tem ganho popularidade na área acadêmica e na indústria, e está agora sendo usado para desenvolver estruturas ótimas no setor automotivo, aeroespacial, aeronáutico, entre outros. Recentemente, o método tem sido aplicado a outros problemas estruturais, como por exemplo, no projeto de materiais com razão de Poisson negativa (materiais que se expandem quando são tracionados) e materiais com coeficientes de expansão térmica negativa (materiais que se contraem quando são aquecidos) (SIGMUND, 2000).

\subsection{Conceitos Básicos}

O MOT (BENDSØE; SIGMUND, 2002) consiste num método computacional que gera a topologia ótima de estruturas. Basicamente, distribui o material no interior de um domínio fixo de forma a maximizar ou minimizar uma função custo especificada (por exemplo, máxima rigidez estrutural ou mínimo volume de material).

A utilização de valores discretos para a variação de material dentro dos elementos finitos, como "zero" para vazio e "um" para sólido, gera uma dependência da solução com a discretização e torna o problema mal-posto, ou seja, sem solução. A solução pode ser obtida através do uso de uma restrição de complexidade, como uma restrição de perímetro (HABER; JOG; BENDSøE, 1996).

No entanto, pela implementação de uma restrição de perímetro ser complexa, outra forma de se resolver esse problema é permitir que o material em cada ponto do domínio possa, por exemplo, variar de um material do tipo A (vazio) a um material do tipo B (um sólido), assumindo materiais intermediários entre A e 
$\mathrm{B}$, de acordo com uma lei de interpolação definida, chamada modelo de material (BENDSØE, 1995). Normalmente, a consideração de mais materiais no problema implica não somente no aumento do número de variáveis de projeto, mas também na formulação de um modelo de material que interpole as propriedades dos materiais em questão (RODRIGUES; SOTO; TAYLOR, 1999). Essas aproximações numéricas contínuas do campo de material binário (vazio e material) são largamente usadas nas implementações de otimização topológica. Um algoritmo de otimização é usado para se determinar, de forma iterativa, a distribuição ótima dos materiais, o que torna o processo rápido. Caso contrário, inúmeras análises seriam necessárias para encontrá-la, dado que cada possibilidade seria testada individualmente. A distribuição de um dado material é representada, por exemplo, associando-se um valor de "densidade" (ou pseudo-densidade, conforme será discutido adiante) a cada elemento obtido da discretização do domínio inicial.

Uma sugestão natural seria resolver o problema através de métodos probabilísticos, como o algoritmo genético. No entanto, este não resolve em si a questão, por ainda se basear no problema mal-posto. O seu uso também não é aconselhado para esse caso por desprezar as informações determinísticas do projeto de estruturas que poderiam auxiliar na convergência para uma solução. Como conseqüência, o método consome tempo computacional com a pesquisa de soluções não factíveis, como topologias desconexas. No entanto, recentemente foram propostos algoritmos de otimização binários de larga escala para o projeto de topologias compostas de material e vazio (BECKERS, 1999; SVANBERG; WERME, 2005).

O MOT implementado nesse trabalho se baseia em dois conceitos fundamentais: o domínio fixo estendido de projeto e o modelo de interpolação de material ou modelo de material.

\subsubsection{Domínio Fixo Estendido de Projeto}

Ao contrário da otimização de forma, no MOT não são alterados os contornos externos do domínio da estrutura durante o processo de otimização. Este domínio fixo que conterá a estrutura desconhecida e que se encontra limitado pelos pontos de apoio da estrutura e os pontos de aplicação dos carregamentos é o chamado "domínio fixo estendido de projeto". Assim, no MOT será removido e adicionado material dentro do espaço definido pelo domínio fixo na procura da topologia ótima, mas a sua forma inicial não é alterada.

Na implementação numérica do módulo de análise, o domínio fixo estendido 
deve ser discretizado em elementos finitos que permanecerão inalterados durante o processo de otimização, sendo alterada somente a distribuição de material nos elementos.

Isto facilita a implementação da análise de sensibilidade, uma vez que vale para todos os elementos do domínio fixo estendido a relação geral do cálculo da sensibilidade:

$$
\frac{\partial}{\partial \rho^{e}} \int_{\Omega} f d \Omega=\int_{\Omega} \frac{\partial f}{\partial \rho^{e}} d \Omega
$$

onde $\rho^{e}$ é a variável de projeto do elemento $e, f$ é uma função contínua e $\Omega$ é o domínio de projeto.

\subsubsection{Modelo de Interpolação de Material}

Como já dito, uma forma de solução para o problema discreto é a relaxação do problema de otimização, que permite que o material em cada elemento do domínio de projeto fixo discretizado assuma valores intermediários durante a otimização ao se passar da condição de ausência de material a sólido. Assim, define-se o conceito do modelo de material, que está relacionado com a forma de se variar o material em cada elemento finito entre "zero" (vazio) e "um" (sólido) e determina a lei de mistura das propriedades dos materiais mediante uma equação. A definição do modelo de material define o grau de relaxação do problema.

Existem vários modelos de material descritos na literatura que podem ser utilizados. Entre os principais destacam-se: o método de homogeneização (MURAT; TARTAR, 1985; BENDSøE; KIKUCHI, 1988) e o método das densidades (BENDSØE, 1989; ZHOU; ROZVANY, 1991; MLEJNEK, 1992). A seguir, uma descrição breve de cada um dos modelos de material para o caso de um material e vazio.

\section{Método de Homogeneização}

O método da homogeneização é baseado em microestruturas formadas pela mistura de materiais homogêneos (BENDSøE; KIKUCHI, 1988). Esse método constitui um modelo de material complexo e robusto para o cálculo das propriedades efetivas de um material composto, conhecida a geometria e composição de sua microestrutura. Assim, tomando como exemplo uma placa perfurada, podemos calcular as propriedades da composição dos materiais 


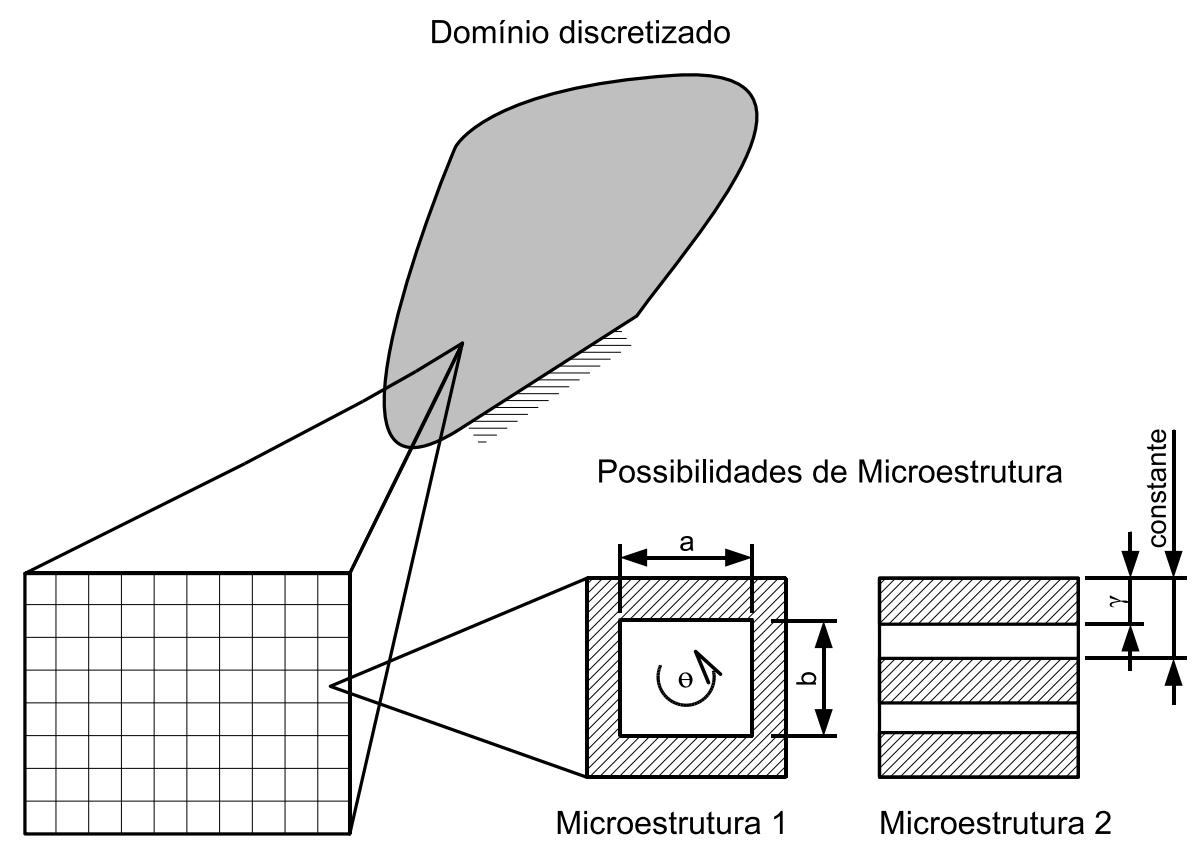

Figura 3.4: Exemplo de Elementos no Método de Homogeneização.

da placa perfurada (sólido e vazio) a partir do material base da placa e conhecendo a distribuição dos furos. Nesse modelo, cada elemento do domínio da estrutura é definido como sendo um material composto gerado pela repetição periódica de uma microestrutura. Desta maneira, existem duas configurações de microestrutura que podem ser utilizadas, a partir das quais podem ser geradas outras microestruturas (FUJII; CHEN; KIKUCHI, 2001). Uma dessas microestruturas é composta por material sólido com vazio interno (microestrutura 1 - figura 3.4) e a outra, por camadas alternadas de material e vazio (microestrutura 2 - figura 3.4).

A microestrutura composta por material sólido com vazio interno consiste numa célula unitária com um buraco retangular no seu interior (BENDSøE; KIKUCHI, 1988; SUZUKI; KIKUCHI, 1991), cujas dimensões são definidas pelas variáveis de projeto $a$ e $b$ e ângulo $\theta$. A microestrutura composta por camadas alternadas de material é construída alternando-se camadas de materiais sólidos com vazios e cujo parâmetro de otimização é a medida $\gamma$. Thomsen (1992), Olhoff, Kog e Thomsen (1993) usaram essa categoria de microestrutura para otimização topológica em seus trabalhos. Assim, define-se um material composto gerado pela repetição periódica de uma microestrutura em cada elemento do domínio

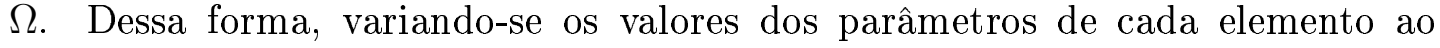
longo do domínio fixo estendido durante a otimização, altera-se a distribuição de material de maneira que, ao final da otimização, existirão elementos vazios, elementos sólidos e alguns pontos com materiais intermediários (mistura entre sólido e vazio). 


\section{Método das Densidades}

O método das densidades, consiste numa equação matemática que define o valor da propriedade do material em cada ponto do domínio em função da densidade (que no problema de otimização é a variável de projeto) e da propriedade básica do material a ser distribuído. Um dos modelos que se encaixam nessa categoria é o "Simple Isotropic Material with Penalization" (SIMP). Este modelo de material pode ser expresso como:

$$
C^{e}=\left(\Phi^{e}\right)^{p} C_{m}
$$

onde $C^{e}$ é a propriedade do material do elemento $e ; \Phi^{e}$ é a densidade relativa de material em um elemento $e$, que está definida no intervalo $0 \leq \Phi^{e} \leq 1 ; p$ é o fator de penalização da variável de projeto; $C_{m}$ é a propriedade do material $m$ considerado.

No modelo de material SIMP, a utilização de um $p$ de menor valor torna o problema mais contínuo do que discreto, o que pode permitir a presença de materiais porosos (ou as chamadas "escalas de cinza") que, além de gerar problemas técnicos na fabricação, não permitem uma adequada interpretação dos resultados. Portanto, é necessário trabalhar com valores de $p$ maiores, que permitem um resultado final mais discreto, com menos densidades intermediárias entre 0 e 1 (problema de natureza mais discreta do que contínua). Caso seu valor seja muito alto, como $p=9$ por exemplo, podem ocorrer problemas de convergência e de instabilidade numérica devido à tendência de retornar ao problema discreto (limitar os valores da densidade de cada elemento somente a 0 ou 1) e, conseqüentemente, mal-posto.

O SIMP também tem sido chamado de aproximação de "material fictício", já que se acreditava que nenhum material alcançaria as propriedades de material modeladas pela equação (3.2). Apesar disso, Bendsøe e Sigmund (1999) provaram que a aproximação SIMP é fisicamente possível, desde que uma condição sobre o $p$ seja satisfeita: o fator de penalização deve ser maior ou igual a 3. Assim, o fator $p$ pode ser ajustado para reduzir as escalas de cinza, sendo a propriedade calculada pela equação (3.2) possível de se obter através de um compósito com a proporção de material indicada se $p$ for selecionado de modo que satisfaça as seguintes relações (em problemas de estado plano de tensão):

$$
\left(\Phi^{e}\right)^{p} E_{m} \leq \frac{\Phi^{e} E_{m}}{3-\Phi^{e}} \quad \text { para } \quad 0 \leq \Phi^{e} \leq 1 \quad \text { e } \quad E_{m} \gg E_{\text {vazio }}
$$




$$
p \geq \max \left\{\frac{2}{1-\nu_{m}}, \frac{4}{1+\nu_{m}}\right\}
$$

onde $E_{m}$ é o módulo de elasticidade e $\nu_{m}$ é o coeficiente de Poisson, ambos referentes ao material $m$ considerado; $E_{v a z i o}$ é o módulo de elasticidade do vazio.

As equações (3.3) e (3.4) são baseadas nos limites de Hashin-Shtrikman (HASHIN; SHTRIKMAN, 1963; BENDSøE; SIGMUND, 1999), os quais definem uma região num gráfico de módulo de elasticidade pela densidade (entre 0 e 1) onde as propriedades de microestruturas formadas a partir da mistura de dois materiais (neste caso, material e vazio) são fisicamente possíveis de serem obtidas. A partir das equações (3.3) e (3.4), pode-se concluir que ambas são satisfeitas se $p \geq 3$ (para o módulo de elasticidade). Deve-se lembrar que valores de $p$ muito altos geram os inconvenientes típicos de natureza discreta (problemas com convergência e instabilidades numéricas).

O método da homogeneização é um método robusto, que gera pouca escala de cinza em seu resultado final, capaz de descrever mais realisticamente as propriedades efetivas de um material homogeneizado a partir da definição de uma célula unitária ortotrópica, mas isso tem um custo: a introdução de novas variáveis de projeto no MOT. De fato, o maior número de variáveis de projeto no problema torna o custo computacional deste método relativamente mais alto, além da maior complexidade da implementação numérica. Diante disso, o método de densidades, do qual o SIMP é um de seus mais conhecidos representantes, tem ganho a preferência dos pesquisadores da área de OT por ser um modelo mais simples de implementar e por utilizar somente uma variável de projeto: a densidade relativa do material em cada ponto do domínio fixo estendido de projeto. Apesar da simplicidade, permite chegar num resultado muito semelhante ao obtido usando o método da homogeneização. Na literatura existem vários artigos científicos que implementam o MOT utilizando esse modelo de material, em particular o SIMP (SIGMUND, 1997, 1998, 2001b, 2001c; JONSMANN; SIGMUND; BOUWSTRA, 1999b, 1999a; YIN; YANG, 2000; PEDERSEN, 2001). Assim, pelas vantagens apresentadas pelo método das densidades, este será o modelo de material implementado, com algumas modificações para que possa representar mais de um material. 


\subsection{Desenvolvimento do MOT}

A seguir, será apresentado um histórico do desenvolvimento do MOT aplicado à otimização estrutural, uma das bases do presente trabalho. Primeiro, descrevese o histórico geral da otimização. Depois, aborda-se o estado da arte das formulações que consideram vários materiais.

\subsubsection{Histórico}

Os primeiros problemas de otimização estrutural, foram resolvidos por Maxwell em 1872 e, posteriormente, por Michell (1904). Michell, baseado no estudo de Maxwell estudou as condições necessárias para uma estrutura formada por barras ter o mínimo volume de material respeitando restrições de tensão. Em seu estudo, calculou o campo de tensão mecânica principais, usando teoria da elasticidade, de uma força aplicada num ponto de um domínio infinito que está sujeito a restrições de deslocamento em outros pontos. Obtidas as linhas de isotensão principais, a idéia básica então, era propor nesse domínio uma estrutura formada por barras (treliça), em que cada barra (elemento de treliça) estivesse alinhada com as direções principais de tensão calculadas no domínio. Ou seja, a estrutura ótima, em que o material fosse melhor aproveitado, seria aquela em que os elementos estariam sujeitos apenas a tração e compressão e não há momentos fletores. Assim, utilizando-se do conceito de alinhar as barras com as tensões principais no domínio, Michell obteve resultados de estruturas de treliça para domínios bi e tridimensionais. Existem infinitas linhas de isotensão principais conectando a força aos suportes mas somente algumas são apresentadas. Verificou-se posteriormente que estes resultados são os mesmos obtidos com o critério de máxima rigidez com mínimo volume de material, considerando um único caso de carga, no MOT. Assim, ficou sendo reconhecido como o primeiro trabalho de otimização topológica de estruturas. O trabalho de Michell permaneceu muitos anos na obscuridade até ser retomado por Cox (1956), Hemp (1973), Owen (1965), Prager (1974), entre outros.

A otimização de meios contínuos se desenvolveu com a introdução dos métodos de relaxação do problema discreto original, através da introdução de materiais compostos, iniciado da década de 70. O conceito foi desenvolvido independentemente por vários grupos de pesquisa, como por exemplo Cheng (1981), Cheng e Olhoff (1982), Murat e Tartar (1985), Lurie, Cherkaev e Fedorov (1982a, 1982b), Raitum (1979), Kohn e Strang (1986a, 1986b, 1986c). 
Cheng e Olhoff (1982) demonstraram que a configuração ótima no problema de otimização da distribuição de espessuras para a maximização da rigidez com restrição de volume em placas finas consistia em regiões com infinitos reforços infinitesimais. Os resultados encontrados por eles indicam que a solução ótima é uma placa composta por regiões com infinitos reforços infinitesimais (nervuras), cujo comportamento é similar a um material composto por infinitas microestruturas. Estes resultados inspiraram a metodologia criada por Bendsøe e Kikuchi (1988), cuja base é a microestrutura do material. Com este trabalho, surge um algoritmo computacional para a OT, o MOT. Este consiste num método computacional que permite projetar a topologia ótima de estruturas segundo certo critério de custo, por exemplo, máxima rigidez com o menor peso. No MOT, o material em cada ponto do domínio pode variar de vazio (sem presença de material) até sólido (total presença de material), podendo assumir densidades intermediárias entre vazio e sólido de acordo com um modelo de material definido, ou modelo de interpolação de material.

O trabalho de Bendsøe e Kikuchi (1988) utilizou um modelo de material baseado no método denominado "método de homogeneização". Esse modelo, além de relaxar o problema de otimização topológica, permitiu obter topologias mais definidas e com menos regiões de material poroso (densidade intermediária) ao final do processo. As regiões de material poroso são de difícil fabricação e de utilidade prática apenas durante a otimização. Detalhes do cálculo da relaxação na otimização estrutural podem ser encontrados nos trabalhos de Kohn e Strang (1986a, 1986b). Bendsøe (1989) descreve várias maneiras alternativas de se conseguir a relaxação mencionada acima através da introdução de um modelo de material baseado na distribuição de densidades na microestrutura, como o chamado de "método de densidades" ou SIMP.

Após essa primeira implementação que utiliza o método de homogeneização para a relaxação do problema (por Bendsøe e Kikuchi (1988)), iniciou-se uma grande expansão do método de otimização topológica de meios contínuos. Assim como Bendsøe e Kikuchi (1988), Suzuki e Kikuchi (1991) implementaram o método para resolver, com sucesso, vários exemplos de otimização estrutural cuja função objetivo do problema era a maximização de rigidez, sujeita à restrição de volume de material. Seguiram-se trabalhos de Bendsøe e Rodrigues (1991), Olhoff, Bendsøe e Rasmussen (1991), que estudaram a possibilidade da integração do MOT aos sistemas de "Computer-Aided Design" (CAD) (ou desenho assistido por computador). Suzuki e Kikuchi (1991) comprovaram o embasamento matemático do método de otimização topológica, reproduzindo o 
resultado analítico obtido por Michell (1904).

\subsubsection{Utilização de múltiplos materiais}

Apesar da generalidade e flexibilidade dos resultados obtidos através do MOT, a distribuição de um único material limitava a gama de possibilidades.

A maioria dos trabalhos encontrados que envolviam múltiplos materiais estavam voltados para a área de projeto de microestrutura de materiais. No trabalho de Sigmund e Torquato (1997), o projeto de microestruturas de materiais com coeficiente de expansão térmica nulo ou negativo é realizado com o uso de duas fases de material isotrópico com coeficiente de expansão térmica positivo e de uma fase representando o vazio. Qi, Kikuchi e Mazumder (2004) também abordaram o projeto de tais microestruturas multifásicas, dando uma atenção especial à manufatura. Assim, aspectos como o projeto do caminho da ferramenta para a fabricação e a difusão na interface entre duas fases são abordados, difusão esta que é necessária para a adesão entre as fases na maioria dos métodos de fabricação e pode reduzir a propriedade efetiva pretendida para o material (neste caso, o coeficiente de expansão térmica negativo). No trabalho de Gibiansky e Sigmund (2000), é projetada a microestrutura de materias visando módulos de elasticidade e de cisalhamento extremos. São usadas três ou mais fases de material isotrópico, incluindo o vazio. Torquato (2000) destaca o MOT como um método promissor para o projeto sistemático de microestruturas de compósitos com as propriedades extremizadas, como nos trabalhos recém-citados.

Uma maneira alternativa de se realizar a otimização de estruturas compostas de múltiplos materiais é através de um modelo denominado "multi-phase levelset". Proposto inicialmente para processamento digital de imagem, Wang e Wang (2004) adaptaram-no à otimização topológica. Ao invés de utilizar algorítmos que avaliam a densidade dos materiais num domínio discreto, a abordagem adotada é a de definir as fronteiras de regiões de cada material. Desse modo, não é necessário interpolar as propriedades dos materiais ou misturar as fases, técnicas de relaxação do problema anteriormente mal posto que podem acabar por gerar partes da estrutura de difícil fabricação (a mistura de material e vazio). Para o uso de um número elevado de materiais (algumas dezenas, por exemplo), pode ser vantajoso o uso dessa técnica, já que o número de funções "level-set" necessárias para representar os múltiplos materiais cresce logaritmicamente, um comportamento muito melhor que o aumento linear das variáveis de projeto encontrado no SIMP descrito por Sigmund (2001c) e na seção 4.2. No trabalho 
de Wang e Wang (2005) é detalhada a abordagem variacional do projeto de objetos heterogêneos (com vários materiais). Vários exemplos bidimensionais são apresentados em ambos os artigos, alguns deles comuns aos dois. Yulin e Xiaoming (2004) também utilizam o método "level-set" para problemas com múltiplos materiais e restrições. Várias alterações no modelo de representação de material são usados na otimização topológica de estruturas rígidas e mecanismos flexíveis a projeto de materiais. É citada uma desvantagem da técnica: a impossibilidade de se gerar interfaces entre os materiais durante o processo de otimização. Outras alterações no processo de otimização são abordadas como uma maneira para superar essa desvantagem e melhorar a eficiência computacional. Esta técnica não será usada neste trabalho.

Além do projeto de microestruturas de materiais ou de estruturas, alguns trabalhos focaram o projeto de mecanismos compostos por mais de um material utilizando o MOT, visto que a possibilidade de se usar adicionalmente outro material na estrutura pode aumentar o desempenho e funcionalidade de um mecanismo em relação ao seu projeto realizado com apenas um material e vazio (SIGMUND, 2001c).

Seguindo o próprio trabalho, Sigmund (2001b), Sigmund (2001c) estende o conceito do MOT aplicado ao projeto de MEMS eletrotermomecânicos compostos de um material (SIGMUND, 2001b) para um onde dois materiais são empregados. O acréscimo de um material no projeto do mecanismo levou ao uso de duas variáveis de projeto por elemento do domínio fixo: uma variável indica o quanto de material e vazio está presente em determinado elemento; a outra serve pra mostrar qual é o balanço entre os dois materiais no elemento. A interpolação das propriedades dos materiais foi feita de uma forma híbrida, com o SIMP (BENDSØE, 1989; ZHOU; ROZVANY, 1991; MLEJNEK, 1992), usado para interpolar entre o vazio e o material composto, e uma média ponderada dos limites superiores e inferiores de Hashin-Shtrikman para interpolar as propriedades dos dois tipos de material (seção 3.2.2). A função objetivo a ser maximizada é o deslocamento de um dado ponto da estrutura contra uma mola de rigidez conhecida (como descrito por Sigmund (1997)), tendo como restrições o volume total, a corrente elétrica máxima e o acoplamento com o deslocamento perpendicular ao de interesse. Um método de filtragem foi utilizado para tornar os resultados mais independentes da malha (seção 3.4.1), consistindo na substituição das sensibilidades de cada elemento por médias ponderadas das sensibilidades das vizinhanças, calculadas através do método adjunto (ver seção 4.3).

Usando um método das densidades (seção 3.2.2), Yin e Ananthasuresh (2001) 
propõem uma nova função, denominada "função de pico", para a interpolação das propriedades dos materiais. Essa abordagem permite implementar o MOT com qualquer número de materiais com apenas uma variável de projeto por elemento. Assim, são projetados mecanismos flexíveis multifásicos, maximizando o deslocamento de um ponto da estrutura numa dada direção devido à aplicação de uma força de entrada em outro determinado ponto, restringindo-se ou não o volume de material utilizado. O deslocamento é feito contra uma mola de rigidez conhecida. É utilizado um esquema de atualização iterativa para resolver esse problema através do método do critério de optimalidade. Vários exemplos apresentados envolvem um material mais rígido e outro mais flexível, mostrando que é possível projetar tais micromecanismos facilmente usando o novo modelo de interpolação de propriedades dos materiais. O artigo também cita algumas técnicas modernas que permitem a fabricação econômica de mecanismos trifásicos, sendo o vazio uma das fases. Ainda utilizando a função de pico, mas resolvendo o problema para o projeto de MEMS eletrotermomecânicos, Yin e Ananthasuresh (2002) consideram não somente a convecção no plano de projeto, mas também a proveniente das laterais dos elementos expostos ao ambiente. É maximizado o deslocamento de um ponto da estrutura, restringindo-se o volume de material. Ao utilizar apenas um material no projeto em alguns exemplos, chegou-se a soluções com estruturas conectadas por elementos com escala de cinza como uma tentativa de se isolar eletricamente determinada região ou empregar um material mais flexível que o permitido para o projeto. O uso de duas fases de material, mais o vazio como uma terceira fase, permitiu que tais regiões fossem representadas por um dos materiais e não mais por um material fictício. As duas fases de material utilizadas eram dotadas de diferentes condutividades elétricas (um deles sendo um isolante) e térmicas, módulos de Young e coeficientes de expansão térmica.

\subsection{Problemas Numéricos}

Existem alguns problemas nos resultados do MOT próprios da sua natureza discreta e o seu caráter computacional, como as escalas de cinza e a instabilidade de tabuleiro. Outros problemas importantes existentes nessa metodologia são a dependência de malha da solução ótima e a não unicidade da solução. Todos estes problemas foram descritos em detalhe por Bendsøe e Sigmund (2002), Sigmund e Petersson (1998). 


\subsubsection{Dependência de malha}

O problema de otimização topológica considerando valores discretos (zero ou um) é mal-posto, ou seja, não apresenta solução. Essencialmente, o que acontece é que a utilização de valores discretos origina uma dependência da solução em relação a discretização do problema. O problema da dependência de malha consiste na obtenção de diferentes soluções para diferentes discretizações do domínio de projeto fixo e tamanhos de malhas. Assim, contrariam o objetivo do refinamento e melhores disposições de malha de elementos finitos que deveriam resultar numa melhor aproximação do problema real e não numa estrutura diferente.

Uma das maneiras de se reduzir a dependência da malha é controlar a variação da quantidade de material entre elementos próximos (dentro de um determinado raio) através de restrições de complexidade como filtros, que também acabam por reduzir a presença da instabilidade de tabuleiro, outro tipo de problema numérico. O problema da instabilidade de tabuleiro e as técnicas de filtragem estão descritas na seção 3.4.4.

\subsubsection{Não unicidade da solução}

O caso específico do projeto de uma estrutura com máxima rigidez e menor peso é por natureza convexo, mas quando o problema de otimização resolvido pelo MOT se aproxima do caso discreto, esse projeto se torna um problema não-convexo, com múltiplas soluções ótimas locais e não uma única global. Utilizando o modelo de material de função de pico, a aproximação do problema discreto se dá através da utilização de desvios padrão relativamente baixos para as funções de pico de cada material, gerando picos mais pronunciados que acabam por penalizar a existência de densidades intermediárias dos materias (ver seção 4.2.2), se aproximando do problema discreto. Em outras palavras, a natureza não-convexa do problema matemático leva a uma não-unicidade da solução, isto é, o ponto ótimo encontrado não é necessariamente o mínimo global (ver figura 3.5).

Assim, a existência de mínimos locais devido à aproximação com o problema discreto conduz o algoritmo a diferentes soluções possíveis, considerando o mesmo tamanho de malha. Portanto, diferentes pontos de partida, parâmetros de filtro e do algoritmo de otimização interferem no resultado obtendo diferentes soluções ótimas. O problema de projetar micromecanismos eletrotermomecânicos, ao contrário do projeto de máxima rigidez e menor peso, já é por natureza nãoconvexo e a não-convexidade, no geral, tende a se agravar com a aproximação do 


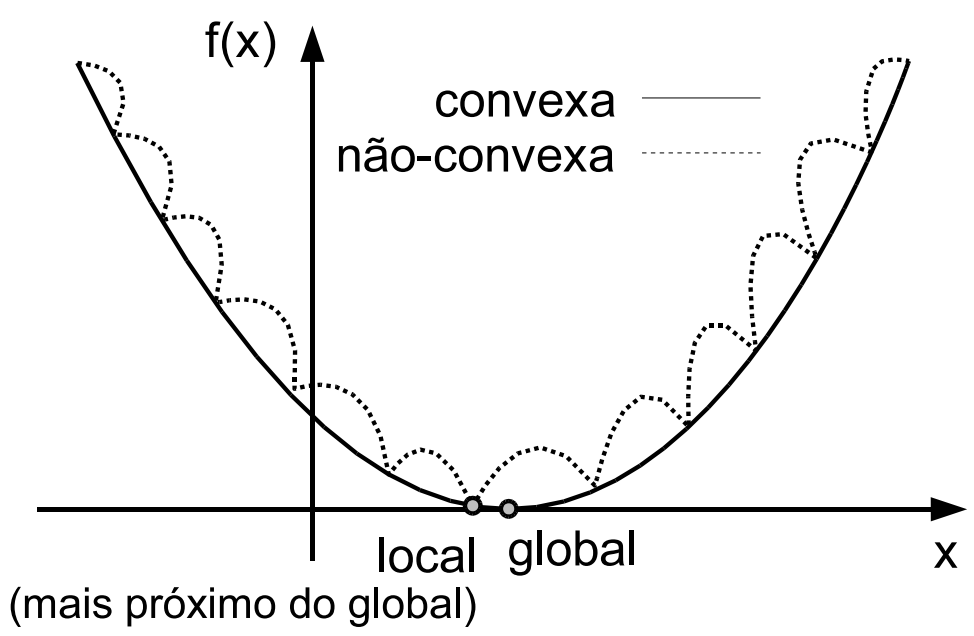

Figura 3.5: Mínimos dos problemas convexo e não-convexo.

problema discreto.

Para tentar diminuir o problema da não-unicidade da solução, utiliza-se o método da continuação (SVANBERG, 1994b, 1994a) no qual a passagem do problema contínuo para o discreto é feita gradualmente. Isso é feito reduzindo-se o grau de relaxação do problema a cada iteração do MOT. Usando o modelo de material de função de pico, isso significa iniciar o processo de otimização com um desvio padrão mais alto e reduzindo-o a cada iteração até que se atinja um valor mínimo pré-estabelecido. Assim, passa-se de um problema mais convexo (curva cheia da figura 3.5) ao menos convexo (curva de linha pontilhada da figura 3.5) de maneira gradual. A idéia é fazer com que, durante o MOT, a solução siga a curva mais convexa com os desvios padrão mais altos até alcançar o mínimo local mais próximo do global, quando os desvios padrão atingem valores mais baixos e o problema se torna bem menos convexo.

\subsubsection{Escalas de cinza (ou "grayscale")}

A utilização de uma relaxação muito alta do problema discreto gera elementos com densidades intermediárias, ou seja, um material com propriedades intermediárias entre sólido e vazio nessas regiões. Isto, do ponto de vista prático, não é interessante, pois dificulta a interpretação final da topologia e torna complicada, ou mesmo inviável, a sua fabricação, já que não é usual obter um material que ofereça uma variação contínua de densidade em toda a estrutura do mecanismo. Assim, é necessário a utilização de valores menores para os desvios padrão do modelo de material de função de pico (figura 4.3), que deve ser ajustado para reduzir o aparecimento das escalas de cinza. Deve-se tomar cuidado para não prejudicar a convergência do problema com valores muito baixos dos desvios 


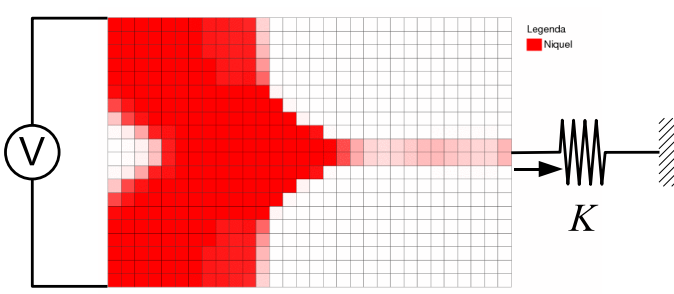

(a) Peça de rigidez $K$

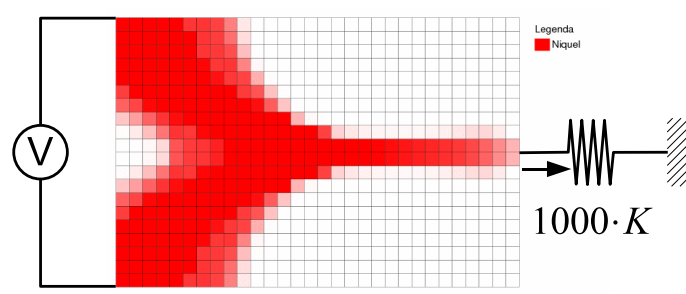

(b) Peça de rigidez $1000 \cdot K$

Figura 3.6: Exemplo de aparecimento de escalas de cinza.

padrão ou uma continuação muito abrupta, que podem acabar por impedir a evolução da topologia.

O aparecimento das escalas de cinza também pode ser a tentativa do MOT de gerar uma topologia de maior deslocamento ao definir uma estrutura mais flexível que a permitida pelos materiais usados. Isso é conseguido pelas densidades intermediárias, o que sacrifica a própria rigidez estrutural do mecanismo e a factibilidade do resultado. Assim, outra maneira de se reduzir as escalas de cinza é considerar uma rigidez $K$ para a peça atuada, aumentando-a conforme seja necessário. Desse modo, o deslocamento de saída é maximizado para um mecanismo capaz de aplicar uma maior força sobre a peça atuada, o que privilegia um resultado com maior integridade estrutural e menos escalas de cinza. $\mathrm{Na}$ seção 1.3 foram citados alguns trabalhos que utilizaram essa abordagem. A figura 3.6 ilustra os resultados obtidos para um problema de maximização dos deslocamentos de saída de um mecanismo eletrotermomecânico, com uma rigidez diferente para cada peça atuada (uma é mil vezes mais rígida que a outra).

\subsubsection{Instabilidade de tabuleiro (ou "checkerboard")}

Esse tipo de instabilidade consiste na formação, na solução ótima, de regiões com elementos sólidos (de densidade "um") e elementos vazios (de densidade "zero"), dispostos em forma de tabuleiro de damas (ou "checkerboard" como é conhecido na literatura) como pode ser visto na figura 3.7.

Este problema ocorre devido à formulação (funções de interpolação) do elemento finito utilizado no processo de otimização, já que as aproximações numéricas introduzidas pelo $\mathrm{MEF}$ fazem com que o arranjo do material em forma de tabuleiro de seja artificialmente mais rígido do que o arranjo uniforme, considerando o mesmo volume de material em ambos os casos (KIKUCHI; HOLLISTER; YOO, 1997; DíAZ; SIGMUND, 1995). Assim, como geralmente a maximização de rigidez é uma característica implícita em vários problemas de 


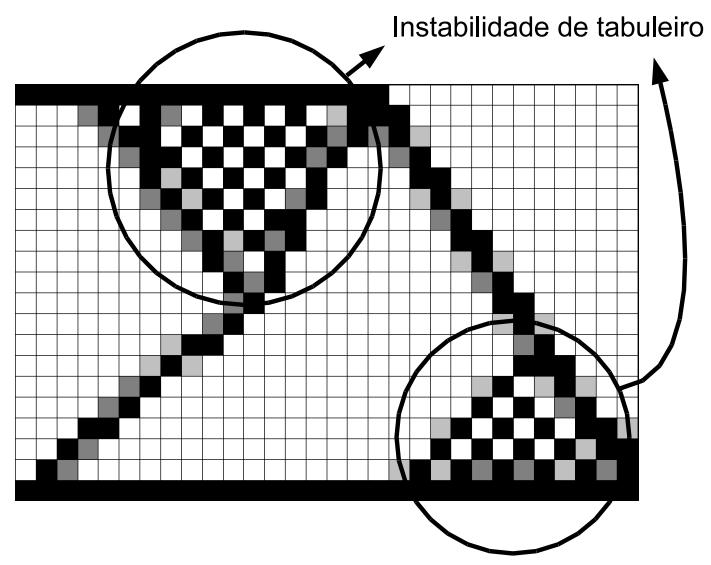

Figura 3.7: Exemplo de aparecimento de instabilidade de tabuleiro.

OT (e explícita no caso do problema de maximização de rigidez com restrição de volume de material) na busca da solução ótima, o MOT acaba optando pela formação da instabilidade de tabuleiro.

O controle da instabilidade de tabuleiro no projeto topológico foi primeiramente discutido por Bendsoe, Diaz e Kikuchi (1993), Jog, Haber e Bendsøe (1993), Rodrigues e Fernandez (1993). Haber, Jog e Bendsøe (1996) propõem uma restrição de perímetro para o controle da complexidade da solução, o que acaba também por reduzir o problema da instabilidade de tabuleiro. Duas outras maneiras para diminuir o "checkerboard" no MOT são: aumentar a ordem do elemento finito e utilizar métodos de filtragem ou de controle de gradientes (DíAZ; SIGMUND, 1995). Uma análise detalhada do problema de instabilidade de xadrez foi descrita por Díaz e Sigmund (1995), Jog e Haber (1996), Bendsøe e Sigmund (2002).

\section{Aumento da ordem do elemento finito}

Aumentar a ordem do elemento significa aumentar o número de nós do elemento finito. Os elementos com 8 ou 9 nós possuem funções de interpolação que representam melhor o campo de deslocamentos no elemento e, conseqüentemente, o campo de deformações. Então, a utilização de elementos com maior número de nós permite reduzir o erro induzido aos termos de deformação de cisalhamento no elemento, e portanto representar de forma menos artificial a rigidez num arranjo de elementos finitos diminuindo, assim, a tendência de formação de "checkerboard".

Petersson e Sigmund (1998) utilizaram essa solução para obter resultados isentos da formação de instabilidade de xadrez para o problema de otimização topológica cuja função objetivo considerava a máxima rigidez de uma estrutura 
com restrição de volume de material. Concluíram que esta é uma alternativa cara devido ao alto custo computacional, pois a matriz de rigidez do modelo de MEF se torna demasiadamente grande a medida que aumenta o número de nós do elemento. Além disso, é importante observar que Díaz e Sigmund (1995) demonstraram que, para determinados valores do fator de penalidade $p$ nos problemas de MOT baseados no SIMP, elementos de 9 nós também podem causar a formação de instabilidade de xadrez.

\section{Técnicas de filtragem}

Pode-se reduzir a formação da instabilidade de tabuleiro ao se utilizar um método de controle sobre a variação espacial das variáveis de projeto. Apesar desse procedimento não eliminar as causas, o controle de variações bruscas nos gradientes das variáveis de projeto ao longo do domínio permite a redução das ocorrências desse tipo de instabilidade. Além disso, esses mesmos métodos permitem um razoável controle da complexidade da topologia obtida pelo MOT (BOURDIN, 2001). Assim, a suavização da variação das variáveis de projeto, por exemplo, nos problemas de OT pode ser feita através de filtragem.

Uma das formas de se classificar os métodos de filtragem utilizados em OT que consideram elementos vizinhos é: filtros de vizinhança fixa, onde somente vizinhos de aresta e/ou nó são considerados, e filtros espaciais, onde elementos que se encontram dentro de um determinado raio de varredura em torno do elemento central são considerados (CARDOSO, 2000).

No caso dos filtros de vizinhança fixa, Swan e Kosaka (1997) propuseram um filtro deste tipo, no qual uma certa grandeza relacionada a um elemento depende da grandeza correspondente relacionada aos elementos vizinhos. Apesar deste filtro ser eficaz para evitar a formação do "checkerboard", ele é dependente da discretização do domínio fixo estendido, ou seja, obtêm-se resultados diferentes conforme aumentamos ou reduzimos essa discretização. Cardoso e Fonseca (1999) propuseram uma versão baseada no filtro de Swan e Kosaka (1997), na qual implementaram um conceito de raio de abrangência no filtro para torná-lo independente do refino da malha do domínio fixo estendido.

O filtro espacial é a técnica mais tradicional e utilizada até agora para se reduzir a instabilidade de tabuleiro na maioria dos trabalhos que envolvem o MOT. Além da filtragem direta sobre as variáveis de projeto (a técnica mas simples e comum), essa filtragem espacial também pode ser aplicada sobre as sensibilidades (SIGMUND, 2001b, 1997), onde a sensibilidade do elemento em 
questão é substituída por uma média ponderada das sensibilidades dos seus vizinhos dentro de um raio dado. Outra forma de realizar a filtragem é fazêlo diretamente sobre os limites móveis (CARDOSO, 2000) quando se trabalha com as técnicas de Programação Linear Sequencial (PLS) ou "Method of Moving Asymptotes" (MMA) (SVANBERG, 1987) para resolver o MOT. A aplicação do filtro sobre os limites móveis apresenta como vantagens a facilidade de implementação e a não modificação da distribuição da variável de projeto e de seus gradientes, além de requerer menos iterações no processo de filtragem. $\mathrm{O}$ inconveniente desta forma de filtragem é a dependência do desempenho do filtro com o método de determinação dos limites móveis, já que variações elevadas ou bruscas dos limites móveis tendem a atenuar o método de filtragem. No entanto, isto não deve ser considerado como um problema, pois grandes variações dos limites móveis não são desejáveis devido à aproximação linear utilizada no PLS e no MMA, conforme será descrito na seção 5.1.

Neste trabalho, a técnica de filtragem espacial sobre os limites móveis foi usada, sendo descrita em detalhes na seção 5.3. 


\section{Formulação do Problema de Otimização de MEMS Eletrotermomecânicos Multifásicos}

Neste capítulo é apresentado o problema de otimização utilizado no projeto de micromecanismos eletrotermomecânicos multifásicos, considerando ou não multiflexibilidade (seção 4.1), e uma descrição do modelo de interpolação para mais de um material, além do vazio (seção 4.2). Também é descrito o cálculo da sensibilidade da função objetivo do problema de otimização em relação às variáveis de projeto (pseudo-densidades), apresentando-se o seu desenvolvimento matemático pelo método adjunto (seção 4.3).

\subsection{Formulação do Problema}

No projeto de micromecanismos eletrotermomecânicos, requisitos cinemáticos e estruturais devem ser considerados. Os primeiros consistem em maximizar a deformação de um ponto específico ao longo de certa direção (a resposta do micromecanismo) quando uma tensão elétrica é aplicada em pontos prédeterminados. Já os requisitos estruturais correspondem a maximizar a rigidez da estrutura quando o mecanismo sofre a reação do objeto manipulado por ele (peça atuada). Assim, com base nas idéias anteriores, o problema do projeto de um micromecanismo eletrotermomecânico consiste em distribuir uma dada quantidade de material num domínio de projeto bidimensional de modo que o deslocamento $u_{\text {saida }}$, gerado sobre uma peça atuada de rigidez $K$, seja maximizado (figura 4.1).

Como a força da atuação está ligada diretamente à topologia do mecanismo, a topologia ótima do atuador dependerá da rigidez da peça atuada. Ajustando-se a rigidez da peça de trabalho, pode-se projetar atuadores orientados a força ou orientados a deslocamento. De um modo geral, micromecanismos mais rígidos 


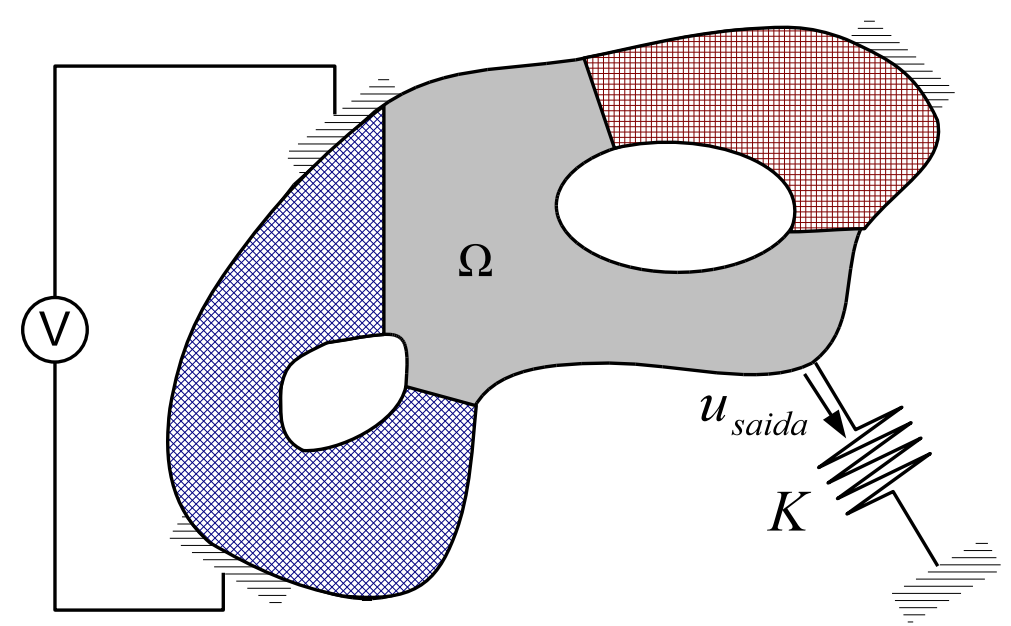

Figura 4.1: Problema do projeto de um micromecanismo eletrotermomecânico.

tendem a ter como resposta deslocamento menores, porém com maior força, enquanto que os mais flexíveis privilegiam o deslocamento em detrimento da força aplicada. Neste trabalho, tenta-se maximizar o deslocamento de saída utilizando uma mola para a modelagem do carregamento de saída (introduzida por Sigmund (1997)), ao invés da maximização da razão de duas energias mútuas baseadas em dois diferentes problemas de elementos finitos, como proposto por Frecker et al. (1997). Na formulação de energia mútua, além de ser voltada para o projeto de mecanismos flexíveis (atuados por força), não existe o mesmo controle da conduta de entrada e saída do micromecanismo e, como comenta Hetrick, Kikuchi e Kota (1999), o problema de otimização apresenta problemas de convergência e dependência do limite mínimo da densidade do elemento.

Com base nesses argumentos, o problema de otimização para o projeto de MEMS eletrotermomecânicos é definido como (SIGMUND, 2001b):

$$
\begin{array}{cc}
\underset{\rho}{\max } F_{E T M}=u_{\text {saida }} & \text { (deslocamento de saída) } \\
\text { tal que } G_{\text {volm }_{m}}=\int_{\Omega} \Phi_{m} d \Omega \leq \Omega_{s_{m}} & (m=1, \ldots, M) \\
& (\text { restrições de volume }- \text { material } m \text { ) }
\end{array}
$$

(Equações de equilíbrio)

onde $F_{E T M}$ é a função objetivo da otimização; $u_{\text {saida }}$ é o deslocamendo de um dado ponto e numa direção determinada; $G_{v o l_{m}}$ é a função que define o volume de um material $m$ na topologia; $\Phi_{m}$ são as densidades relativas de um material $m$ (entre zero e um) de cada ponto do domínio fixo estendido; $\Omega$ é o domínio fixo estendido; $\Omega_{s_{m}}$ é o volume máximo permitido para um material $m$; $M$ é o número 


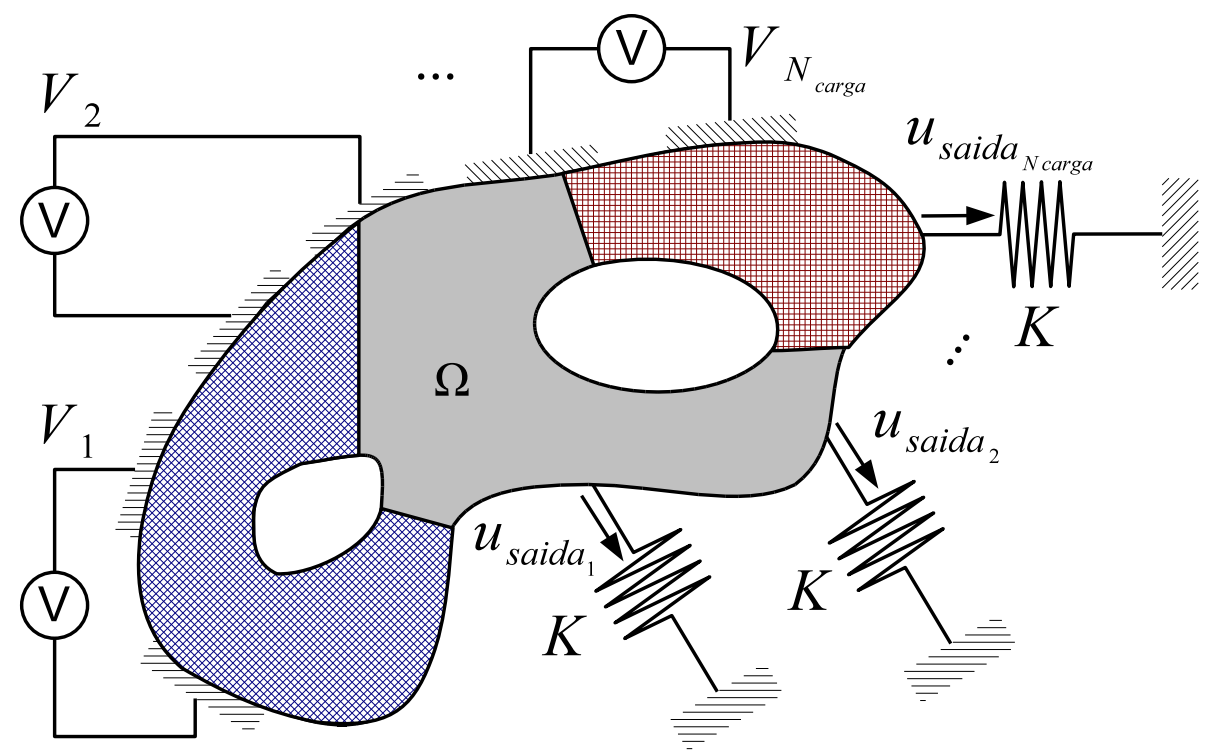

Figura 4.2: Problema do projeto de um micromecanismo eletrotermomecânico multiflexível.

de materiais, além do vazio, considerado na análise. As equações de equilíbrio estão apresentadas de (2.1) a (2.3).

Apesar das densidades relativas $\Phi_{m}$ estarem limitadas a valores entre zero e um, não é necessário haver uma restrição de caixa já que estas não são as variáveis de projeto. As variáveis de projeto, no caso do modelo de material de função de pico, são as pseudo-densidades que podem assumir qualquer valor (ver seção 4.2 sobre modelos de material).

Para o projeto de micromecanismos eletrotermomecânicos multiflexíveis, o problema de otimização enunciado na equação (4.1) foi alterado para que o resultado fosse um micromecanismo que permite $N_{\text {carga }}$ atuações, com cada atuação $c$ relacionada a sua respectiva $u_{\text {saida }_{c}}$ (figura 4.2).

A função objetivo para o problema que considera multiflexibilidade foi formulada a partir de uma modificação da função Kreisselmeier-Steinhauser (KS), de modo que esta assumisse o menor valor de um conjunto de dados (NISHIWAKI et al., 2001; MLEJNEK; SCHIRRMACHER, 1993). Além da função objetivo, as equações de equilíbrio também se alteraram, satisfazendo cada um dos casos de carga. Assim, o problema de otimização passa a ser: 


$$
\begin{array}{cc}
\max _{\boldsymbol{\rho}} F_{E T M_{\text {multi }}}=-\frac{1}{\psi} \ln \left(\sum_{c=1}^{N_{\text {carga }}} e^{-\psi u_{\text {saidac }}}\right) & \text { (menor deslocamento de saída) } \\
\text { tal que } G_{v o l_{m}}=\int_{\Omega} \Phi_{m} d \Omega \leq \Omega_{s_{m}} & (m=1, \ldots, M)
\end{array}
$$$$
\text { (restrições de volume - material } m \text { ) }
$$

(Equações de equilíbrio

para cada caso de carga)

onde $F_{E T M_{m u l t i}}$ é a função objetivo da otimização; $\psi$ é o parâmetro de normalização da função KS; $u_{s a i d a_{c}}$ é o deslocamendo de um dado ponto e numa direção determinada para o caso de carga $c ; N_{\text {carga }}$ é o número de casos de carga. As equações de equilíbrio estão apresentadas de (2.1) a (2.3) e são resolvidas para cada caso de carga.

O parâmetro $\psi$ define a aproximação da função KS em relação ao menor valor do conjunto de dados: quanto maior, melhor a aproximação, sendo o seu valor máximo limitado por razões numéricas (MLEJNEK; SCHIRRMACHER, 1993).

\subsection{Modelos de Interpolação para Múltiplos Materiais}

Conforme já discutido na seção 3.2.2, o modelo de material escolhido para uso neste trabalho foi o método das densidades. Serão descritas duas possibilidades: uma extensão do "Simple Isotropic Material with Penalization" (SIMP) para dois materiais, além do vazio, acrescentando uma variável de projeto por elemento; utilização de uma função de pico.

\subsubsection{Extensão do "Simple Isotropic Material with Penalization" (SIMP)}

A extensão do SIMP para mais um material (totalizando dois materiais, além do vazio) pode ser feita de uma maneira simples, acrescentando-se uma variável de projeto por elemento:

$$
C^{e}\left(\Phi_{A}^{e}, \Phi_{B}^{e}\right)=\left(\Phi_{A}^{e}\right)^{p}\left[\left(\Phi_{B}^{e}\right)^{p} C_{m=1}+\left(1-\Phi_{B}^{e}\right)^{p} C_{m=2}\right]+\left(1-\Phi_{A}^{e}\right)^{p} C_{v a z i o}
$$

onde $\Phi_{A}^{e}$ é a densidade relativa entre sólido (composto) e vazio, $\Phi_{B}^{e}$ é a densidade 
relativa entre o material 1 e 2 , na parte sólida. $p$ é o fator de penalização das variáveis de projeto. $C_{m=1}$ e $C_{m=2}$ são as propriedades dos materiais 1 e 2 a serem interpoladas pelo modelo, $C_{\text {vazio }}$ é a propriedade do vazio, e $C^{e}$ é o resultado da interpolação das propriedades. As propriedades a serem interpoladas podem ser: elementos da matriz de elasticidade $\boldsymbol{D}$ (calculada a partir das propriedades de cada material), coeficiente de expansão térmica $\alpha$, condutividade elétrica $\sigma_{0}$ ou condutividade térmica $\sigma_{1}$.

Essa formulação foi sugerida e utilizada com sucesso por Gibiansky e Sigmund (2000), Sigmund e Torquato (1997), apesar dela possuir alguns problemas, como violar os limites de Hashin-Shtrikman (ver seção 3.2.2) para baixos valores de $\Phi_{B}^{e}$ e maiores valores de $p$, e mudar de comportamento quando os materiais 1 e 2 são trocados entre si (SIGMUND, 2001c). Como o objetivo da otimização é obter estruturas com materiais bem definidos (sem materiais mistos), não haveria problemas decorrentes da violação dos limites de Hashin-Shtrikman, que dizem respeito somente a compósitos.

Se quiser que estes limites sejam respeitados durante o processo de otimização, a utilização de um modelo de material dado pela média ponderada dos limites superior e inferior de Hashin-Shtrikman para cada propriedade dos materiais é uma alternativa, como descrito por Sigmund (2001c).

\subsubsection{Função de Pico}

A extensão do SIMP para mais materiais tem o inconveniente de acrescentar uma variável de projeto por elemento para cada material adicional, o que aumenta o custo computacional e a memória requerida para o processamento. Por exemplo, ao acrescentar um material ao SIMP com um material e vazio, dobra-se o número de variáveis de projetos, que tem impacto principalmente na análise de sensibilidades (seção 4.3) e no módulo de otimização (a PLS, seção 5.1.1), aumentando tanto o tempo computacional como a memória para armazenar as informações necessárias.

Outra maneira de se conseguir uma interpolação suave entre os materiais é utilizar uma combinação linear de funções semelhantes à distribuição gaussiana (YIN; ANANTHASURESH, 2002):

$$
C^{e}\left(\rho^{e}\right)=\sum_{m=1}^{M} C_{m} e^{-\left(\rho^{e}-\mu_{m}\right)^{2} / 2 \sigma_{m}^{2}}+C_{v a z i o}
$$

onde $C^{e}$ é a propriedade já interpolada, $C_{v a z i o}$ é a propriedade do vazio e $C_{m}$ 
Exemplo de Propriedade Interpolada (Função de Pico)

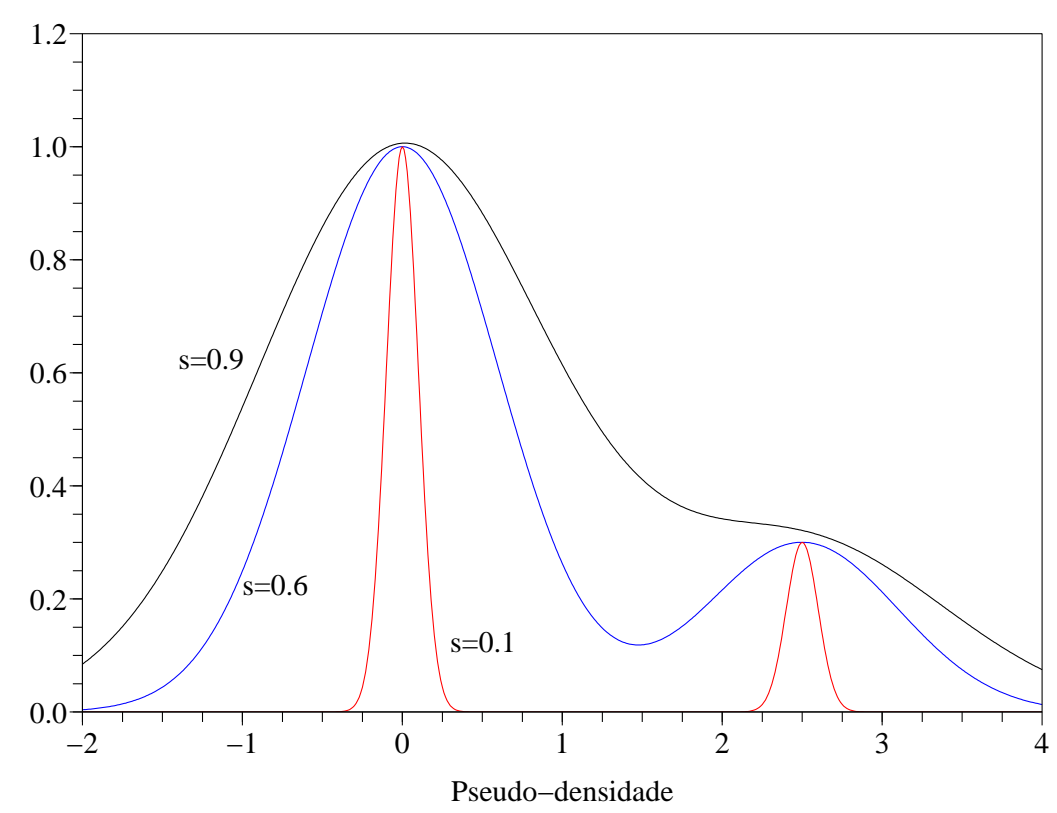

Figura 4.3: Exemplo de Propriedade Interpolada pela função de pico $C_{\text {vazio }}=0, C_{m=1}=1, C_{m=2}=0,3, \mu_{m=1}=0$ e $\mu_{m=2}=2,5$, para três valores diferentes de $s=\sigma_{m=1}=\sigma_{m=2}$.

é a propriedade do material $m$ a ser considerada. $\mu_{m}$ e $\sigma_{m}$ são os parâmetros da distribuição de Gauss de cada termo. $\rho^{e}$ é a pseudo-densidade do elemento (variável de projeto). As propriedades que podem ser interpoladas são as mesmas do caso da extensão do SIMP.

Conforme visto na equação (4.4), uma vantagem desse método é o uso de apenas uma variável de projeto por elemento para qualquer número de materiais considerados na otimização.

Sabendo que cada termo em separado é uma distribuição de Gauss, $\mu_{m}$ representa a média, ou seja, o valor da densidade $\rho^{e}$ onde ocorrerá o pico, e $\sigma_{m}$ representa o desvio padrão, usado para controlar a suavidade com que o pico é formado pelo termo. A amplitude do pico gerado por cada um dos termos é dado pelo peso da combinação linear, que é, neste caso, a propriedade do material representado. Assim, plotando $C^{e}$ dado pela equação (4.4) ao longo de $\rho^{e}$, obtémse vários picos com os valores das propriedades de cada material desde que o valor de $\sigma_{m}$ seja baixo o suficiente para que um termo não interfira no pico vizinho. Um exemplo de função de pico interpoladora para dois materiais e vazio pode ser visto na figura 4.3 .

Visto o comportamento das curvas, inicia-se a otimização com valores relativamente altos para os vários $\sigma_{m}$, o que nos dá uma transição mais suave entre os valores de $\rho^{e}$ que denotam uma propriedade $C_{\text {vazio }}$ (próxima de um 
valor nulo) e os que determinam propriedades como $C_{m}$ nos picos. Reduzindo os $\sigma_{m}$ gradativamente, obtém-se o mesmo efeito de aumentar a penalização no SIMP, evidenciando os picos e reduzindo valores intermediários de valores da propriedade, chegando ao fim da otimização com a maioria dos elementos com propriedade $C_{v a z i o}$ ou $C_{m}$. Este processo é também conhecido como continuação. Como $C^{e}$ tende a $C_{v a z i o}$ quando $\rho^{e}$ tende a $-\infty$ e $\infty$, pode-se dizer que o problema possui limites intrínsecos, o que permite dispensar as restrições de $\rho^{e}$ entre valores definidos, como zero e um no caso do SIMP.

Este método apresenta algumas potenciais desvantagens devido à sua própria definição. Ao alocar os valores de $\mu_{m}$, pode-se também estar definindo as fronteiras entre os materiais. Por exemplo, no caso de três materiais considerados na otimização, sendo $\mu_{m=1}<\mu_{m=2}<\mu_{m=3}$, será mais provável existirem interfaces entre os materiais 1 e 2 que entre 1 e 3 . No caso da extensão do SIMP, essa possibilidade não existe, existindo interfaces igualmente prováveis entre quaisquer pares de material.

Ao longo deste trabalho, será usado o modelo de material baseado na função de pico.

\subsection{Análise de sensibilidades}

Os gradientes da função objetivo e restrições são chamados de sensibilidades do problema de otimização. O cálculo desses gradientes é importante devido à necessidade da linearização da função objetivo em relação às variáveis de projeto, em particular, ao solucionar o problema de otimização mediante o método de Programação Linear Sequencial (PLS), que será abordado na seção 5.1.1.

Existem três métodos para o cálculo de sensibilidade: Método de Diferenças Finitas (MDF), método semi-analítico e método analítico (HAFTKA; GURDAL; KAMAT, 1990). A precisão de cálculo aumenta do primeiro para o último, razão pela qual prefere-se trabalhar com o método analítico, mas comprovando a sensibilidade do problema utilizando o MDF apenas na implementação. Os métodos analíticos podem, por sua vez, ser divididos em método direto e método adjunto. Neste trabalho, utiliza-se o método adjunto.

No método direto, a sensibilidade é determinada de forma explícita para cada um dos subproblemas de MEF envolvido na análise de um micromecanismo eletrotermomecânico; assim, primeiro é determinada a sensibilidade do problema elétrico, que serve de base para determinar a sensibilidade do problema 
eletrotérmico e, analogamente, a partir deste último são obtidos dados para encontrar a sensibilidade do problema termoelástico, determinando-se com isto as sensibilidades procuradas.

O método adjunto produz o mesmo resultado que o método direto, mas do ponto de vista computacional existem diferenças. O método direto é mais eficiente quando o número de variáveis de projeto é menor do que o número de restrições não-lineares. Já o método adjunto é mais eficiente quando número de variáveis de projeto é maior do que o número de restrições não-lineares (HAFTKA; GURDAL; KAMAT, 1990). O problema do projeto de micromecanismos eletrotermomecânicos encaixa-se evidentemente neste último grupo, já que as restrições não-lineares são no máximo as restrições de volume (uma para cada material considerado na otimização), mas as variáveis de projeto dependem da discretização do domínio de projeto e podem ser da ordem de milhares.

\subsubsection{Sensibilidade através do método adjunto}

Assim, para as funções objetivo $F_{E T M}$ e $F_{E T M_{m u l t i}}$ dos dois problemas de otimização enunciados (equações (4.1) e (4.2)), as derivadas totais em relação a uma variável de projeto $\rho^{e}$, a pseudo-densidade de um elemento $e$ qualquer, são:

$$
\begin{gathered}
F_{E T M}=u_{\text {saida }} \\
\frac{d F_{E T M}}{d \rho^{e}}=\frac{d u_{\text {saida }}}{d \rho^{e}} \\
\frac{d F_{E T M_{\text {mult }}}}{d \rho^{e}}=\frac{d}{d \rho^{e}}\left[-\frac{1}{\psi} \ln \left(\sum_{c=1}^{N_{\text {carga }}} e^{-\psi u_{\text {saidac }}}\right)\right] \\
\left.\frac{d F_{E T M_{\text {multi }}}}{d \rho^{e}}=-\frac{1}{\psi} \ln \left(\sum_{c=1}^{N_{\text {carga }}} e^{-\psi u_{\text {saida }}}\right)\right] \\
\sum_{c=1}^{N_{\text {carga }}} e^{-\psi u_{\text {saidac }}} \sum_{c=1}^{N_{\text {carga }}}\left[e^{-\psi u_{\text {saidac }}}\left(-\psi \frac{d u_{\text {saida }}}{d \rho^{e}}\right)\right]
\end{gathered}
$$

onde $u_{\text {saida }}$ é o deslocamento no grau de liberdade de saída; $u_{s a i d a_{c}}$ é $u_{\text {saida }}$ calculado para um caso de carga $c ; N_{\text {carga }}$ é o número de casos de carga.

As duas funções objetivo dependem da derivada dos deslocamentos de saída em relação a $\rho^{e}$. Ao considerar multiflexibilidade, cada $u_{s a i d a_{c}}$ nada mais é que um deslocamento de saída $u_{\text {saida }}$ para o caso de carga $c$, com suas respectivas condições de contorno. Assim, o cálculo das sensibilidades de cada $u_{s a i d a c}$ 
é o mesmo das sensibilidades de $u_{\text {saida }}$, sendo suficiente explicitar apenas o desenvolvimento das sensibilidades para $u_{\text {saida }}$. Desse modo, tem-se:

$$
\begin{gathered}
u_{\text {saida }}=\boldsymbol{L}_{\mathbf{2}}{ }^{t} \boldsymbol{U}_{\mathbf{2}} \\
\frac{d u_{\text {saida }}}{d \rho^{e}}=\boldsymbol{L}_{\mathbf{2}}{ }^{t} \frac{d \boldsymbol{U}_{\mathbf{2}}}{d \rho^{e}}
\end{gathered}
$$

onde $u_{\text {saida }}$ é o deslocamento no grau de liberdade de saída, $\boldsymbol{L}_{2}$ é o vetor que mascara o deslocamento de saída do restante (contém zeros em todas as posições, exceto na que corresponde ao grau de liberdade de saída), $\boldsymbol{U}_{2}$ é o vetor de deslocamentos nodais.

Considerando a equação de equilíbrio do MEF para o domínio termoelástico (equação (2.6)) e as respectivas derivações em relação a $\rho^{e}$ :

$$
\begin{gathered}
\boldsymbol{K}_{\mathbf{2}}(\boldsymbol{\rho}) \boldsymbol{U}_{\mathbf{2}}(\boldsymbol{\rho})=\boldsymbol{P}_{\mathbf{2}}\left(\boldsymbol{U}_{\mathbf{1}}(\boldsymbol{\rho}), \boldsymbol{\rho}\right) \\
\frac{d\left[\boldsymbol{K}_{\mathbf{2}}(\boldsymbol{\rho}) \boldsymbol{U}_{\mathbf{2}}(\boldsymbol{\rho})\right]}{d \rho^{e}}=\frac{d \boldsymbol{P}_{\mathbf{2}}\left(\boldsymbol{U}_{\mathbf{1}}(\boldsymbol{\rho}), \boldsymbol{\rho}\right)}{d \rho^{e}} \\
\frac{d \boldsymbol{K}_{\mathbf{2}}(\boldsymbol{\rho})}{d \rho^{e}} \boldsymbol{U}_{\mathbf{2}}(\boldsymbol{\rho})+\boldsymbol{K}_{\mathbf{2}}(\boldsymbol{\rho}) \frac{d \boldsymbol{U}_{\mathbf{2}}(\boldsymbol{\rho})}{d \rho^{e}}=\frac{d \boldsymbol{P}_{\mathbf{2}}\left(\boldsymbol{U}_{\mathbf{1}}(\boldsymbol{\rho}), \boldsymbol{\rho}\right)}{d \rho^{e}} \\
\frac{d \boldsymbol{U}_{\mathbf{2}}(\boldsymbol{\rho})}{d \rho^{e}}=\boldsymbol{K}_{\mathbf{2}}(\boldsymbol{\rho})^{-1}\left(\frac{d \boldsymbol{P}_{\mathbf{2}}\left(\boldsymbol{U}_{\mathbf{1}}(\boldsymbol{\rho}), \boldsymbol{\rho}\right)}{d \rho^{e}}-\frac{d \boldsymbol{K}_{\mathbf{2}}(\boldsymbol{\rho})}{d \rho^{e}} \boldsymbol{U}_{\mathbf{2}}(\boldsymbol{\rho})\right)
\end{gathered}
$$

onde $\boldsymbol{K}_{\mathbf{2}}$ é a matriz de rigidez, $\boldsymbol{P}_{2}$ é o vetor de carga estrutural e $\boldsymbol{U}_{\mathbf{1}}$ é o vetor de temperaturas nodais.

Substituindo a equação (4.9) na equação (4.8):

$$
\frac{d u_{\text {saida }}}{d \rho^{e}}=\boldsymbol{L}_{\mathbf{2}}{ }^{t} \boldsymbol{K}_{\mathbf{2}}{ }^{-1}\left(\frac{d \boldsymbol{P}_{\mathbf{2}}}{d \rho^{e}}-\frac{d \boldsymbol{K}_{\mathbf{2}}}{d \rho^{e}} \boldsymbol{U}_{\mathbf{2}}\right)
$$

Derivando-se $\boldsymbol{P}_{\mathbf{2}}\left(\boldsymbol{U}_{\mathbf{1}}(\boldsymbol{\rho}), \boldsymbol{\rho}\right)$ em relação a $\rho^{e}$ e aplicando a regra da cadeia:

$$
\frac{d \boldsymbol{P}_{\mathbf{2}}\left(\boldsymbol{U}_{\mathbf{1}}(\boldsymbol{\rho}), \boldsymbol{\rho}\right)}{d \rho^{e}}=\frac{\partial \boldsymbol{P}_{\mathbf{2}}\left(\boldsymbol{U}_{\mathbf{1}}(\boldsymbol{\rho}), \boldsymbol{\rho}\right)}{\partial \boldsymbol{U}_{\mathbf{1}}} \frac{d \boldsymbol{U}_{\mathbf{1}}(\boldsymbol{\rho})}{d \rho^{e}}+\frac{\partial \boldsymbol{P}_{\mathbf{2}}\left(\boldsymbol{U}_{\mathbf{1}}(\boldsymbol{\rho}), \boldsymbol{\rho}\right)}{\partial \rho^{e}}
$$

Substituindo a equação (4.11) em (4.10):

$$
\frac{d u_{\text {saida }}}{d \rho^{e}}=\boldsymbol{L}_{\mathbf{2}}{ }^{t} \boldsymbol{K}_{\mathbf{2}}{ }^{-1}\left(\frac{\partial \boldsymbol{P}_{\mathbf{2}}}{\partial \boldsymbol{U}_{\mathbf{1}}} \frac{d \boldsymbol{U}_{\mathbf{1}}}{d \rho^{e}}+\frac{\partial \boldsymbol{P}_{\mathbf{2}}}{\partial \rho^{e}}-\frac{d \boldsymbol{K}_{\mathbf{2}}}{d \rho^{e}} \boldsymbol{U}_{\mathbf{2}}\right)
$$

Considerando as formulações do MEF para o domínio eletrotérmico (equação 
$(2.5))$ e as respectivas derivações em relação a $\rho^{e}$ :

$$
\begin{gathered}
\boldsymbol{K}_{\mathbf{1}}(\boldsymbol{\rho}) \boldsymbol{U}_{\mathbf{1}}(\boldsymbol{\rho})=\boldsymbol{P}_{\mathbf{1}}\left(\boldsymbol{U}_{\mathbf{0}}(\boldsymbol{\rho}), \boldsymbol{\rho}\right) \\
\frac{d\left[\boldsymbol{K}_{\mathbf{1}}(\boldsymbol{\rho}) \boldsymbol{U}_{\mathbf{1}}(\boldsymbol{\rho})\right]}{d \rho^{e}}=\frac{d \boldsymbol{P}_{\mathbf{1}}\left(\boldsymbol{U}_{\mathbf{0}}(\boldsymbol{\rho}), \boldsymbol{\rho}\right)}{d \rho^{e}} \\
\frac{d \boldsymbol{K}_{\mathbf{1}}(\boldsymbol{\rho})}{d \rho^{e}} \boldsymbol{U}_{\mathbf{1}}(\boldsymbol{\rho})+\boldsymbol{K}_{\mathbf{1}}(\boldsymbol{\rho}) \frac{d \boldsymbol{U}_{\mathbf{1}}(\boldsymbol{\rho})}{d \rho^{e}}=\frac{d \boldsymbol{P}_{\mathbf{1}}\left(\boldsymbol{U}_{\mathbf{0}}(\boldsymbol{\rho}), \boldsymbol{\rho}\right)}{d \rho^{e}} \\
\frac{d \boldsymbol{U}_{\mathbf{1}}(\boldsymbol{\rho})}{d \rho^{e}}=\boldsymbol{K}_{\mathbf{1}}(\boldsymbol{\rho})^{-1}\left(\frac{d \boldsymbol{P}_{\mathbf{1}}\left(\boldsymbol{U}_{\mathbf{0}}(\boldsymbol{\rho}), \boldsymbol{\rho}\right)}{d \rho^{e}}-\frac{d \boldsymbol{K}_{\mathbf{1}}(\boldsymbol{\rho})}{d \rho^{e}} \boldsymbol{U}_{\mathbf{1}}(\boldsymbol{\rho})\right)
\end{gathered}
$$

onde $\boldsymbol{K}_{\mathbf{1}}$ é a matriz de condutividade térmica, $\boldsymbol{P}_{\mathbf{1}}$ é o vetor de carga térmica e $\boldsymbol{U}_{0}$ é o vetor de tensões elétricas nodais.

Substituindo a equação (4.13) em (4.12):

$$
\begin{array}{r}
\frac{d u_{\text {saida }}}{d \rho^{e}}=\boldsymbol{L}_{\mathbf{2}}{ }^{t} \boldsymbol{K}_{\mathbf{2}}{ }^{-1}\left[\frac{\partial \boldsymbol{P}_{\mathbf{2}}}{\partial \boldsymbol{U}_{\mathbf{1}}} \boldsymbol{K}_{\mathbf{1}}{ }^{-1}(\right. \\
\left.\frac{d \boldsymbol{P}_{\mathbf{1}}}{d \rho^{e}}-\frac{d \boldsymbol{K}_{\mathbf{1}}}{d \rho^{e}} \boldsymbol{U}_{\mathbf{1}}\right)+ \\
\left.+\frac{\partial \boldsymbol{P}_{\mathbf{2}}}{\partial \rho^{e}}-\frac{d \boldsymbol{K}_{\mathbf{2}}}{d \rho^{e}} \boldsymbol{U}_{\mathbf{2}}\right]
\end{array}
$$

Derivando-se $\boldsymbol{P}_{\mathbf{1}}\left(\boldsymbol{U}_{\mathbf{0}}(\boldsymbol{\rho})\right.$ em relação a $\rho^{e}$ e aplicando a regra da cadeia:

$$
\frac{d \boldsymbol{P}_{\mathbf{1}}\left(\boldsymbol{U}_{\mathbf{0}}(\boldsymbol{\rho}), \boldsymbol{\rho}\right)}{d \rho^{e}}=\frac{\partial \boldsymbol{P}_{\mathbf{1}}\left(\boldsymbol{U}_{\mathbf{0}}(\boldsymbol{\rho}), \boldsymbol{\rho}\right)}{\partial \boldsymbol{U}_{\mathbf{0}}} \frac{d \boldsymbol{U}_{\mathbf{0}}(\boldsymbol{\rho})}{d \rho^{e}}+\frac{\partial \boldsymbol{P}_{\mathbf{1}}\left(\boldsymbol{U}_{\mathbf{0}}(\boldsymbol{\rho}), \boldsymbol{\rho}\right)}{\partial \rho^{e}}
$$

Substituindo a equação (4.15) em (4.14):

$$
\begin{array}{r}
\frac{d u_{\text {saida }}}{d \rho^{e}}=\boldsymbol{L}_{\mathbf{2}}{ }^{t} \boldsymbol{K}_{\mathbf{2}}{ }^{-1}\left[\frac { \partial \boldsymbol { P } _ { \mathbf { 2 } } } { \partial \boldsymbol { U } _ { \mathbf { 1 } } } \boldsymbol { K } _ { \mathbf { 1 } } { } ^ { - 1 } \left(\frac{\partial \boldsymbol{P}_{\mathbf{1}}}{\partial \boldsymbol{U}_{\mathbf{0}}} \frac{d \boldsymbol{U}_{\mathbf{0}}}{d \rho^{e}}+\right.\right. \\
\left.+\frac{\partial \boldsymbol{P}_{\mathbf{1}}}{\partial \rho^{e}}-\frac{d \boldsymbol{K}_{\mathbf{1}}}{d \rho^{e}} \boldsymbol{U}_{\mathbf{1}}\right)+ \\
\left.+\frac{\partial \boldsymbol{P}_{\mathbf{2}}}{\partial \rho^{e}}-\frac{d \boldsymbol{K}_{\mathbf{2}}}{d \rho^{e}} \boldsymbol{U}_{\mathbf{2}}\right]
\end{array}
$$

Considerando as formulações do MEF para o domínio elétrico (equação (2.4)) e as respectivas derivações em relação a $\rho^{e}$ :

$$
\begin{gathered}
\boldsymbol{K}_{\mathbf{0}}(\boldsymbol{\rho}) \boldsymbol{U}_{\mathbf{0}}(\boldsymbol{\rho})=\boldsymbol{P}_{\mathbf{0}}(\boldsymbol{\rho}) \\
\frac{d\left[\boldsymbol{K}_{\mathbf{0}}(\boldsymbol{\rho}) \boldsymbol{U}_{\mathbf{0}}(\boldsymbol{\rho})\right]}{d \rho^{e}}=\frac{d \boldsymbol{P}_{\mathbf{0}}(\boldsymbol{\rho})}{d \rho^{e}} \\
\frac{d \boldsymbol{K}_{\mathbf{0}}(\boldsymbol{\rho})}{d \rho^{e}} \boldsymbol{U}_{\mathbf{0}}(\boldsymbol{\rho})+\boldsymbol{K}_{\mathbf{0}}(\boldsymbol{\rho}) \frac{d \boldsymbol{U}_{\mathbf{0}}(\boldsymbol{\rho})}{d \rho^{e}}=\frac{d \boldsymbol{P}_{\mathbf{0}}(\boldsymbol{\rho})}{d \rho^{e}}
\end{gathered}
$$




$$
\frac{d \boldsymbol{U}_{\mathbf{0}}(\boldsymbol{\rho})}{d \rho^{e}}=\boldsymbol{K}_{\mathbf{0}}(\boldsymbol{\rho})^{-1}\left(\frac{d \boldsymbol{P}_{\mathbf{0}}(\boldsymbol{\rho})}{d \rho^{e}}-\frac{d \boldsymbol{K}_{\mathbf{0}}(\boldsymbol{\rho})}{d \rho^{e}} \boldsymbol{U}_{\mathbf{0}}(\boldsymbol{\rho})\right)
$$

onde $\boldsymbol{K}_{\mathbf{0}}$ é a matriz de condutividade elétrica e $\boldsymbol{P}_{\mathbf{0}}$ é o vetor de carga elétrica (correntes elétricas nodais) que pode ou não depender das variáveis de projeto, de acordo com o tipo de excitação inicial do mecanismo. Se excitado por uma corrente elétrica prescrita, $\boldsymbol{P}_{\mathbf{0}}$ é constante e independente das variáveis de projeto, sendo nula a sua derivada em relação a $\rho^{e}$. Se excitado por tensão elétrica, $\boldsymbol{P}_{\mathbf{0}}$ passa a ser depedente de $\rho$. Este último caso, por ser mais genérico, será o considerado nas derivações a seguir.

Substituindo a equação (4.17) em (4.16):

$$
\begin{aligned}
\frac{d u_{\text {saida }}}{d \rho^{e}}=\boldsymbol{L}_{\mathbf{2}}{ }^{t} \boldsymbol{K}_{\mathbf{2}}{ }^{-1}\left\{\frac { \partial \boldsymbol { P } _ { \mathbf { 2 } } } { \partial \boldsymbol { U } _ { \mathbf { 1 } } } \boldsymbol { K } _ { \mathbf { 1 } } { } ^ { - 1 } \left[\frac{\partial \boldsymbol{P}_{\mathbf{1}}}{\partial \boldsymbol{U}_{\mathbf{0}}} \boldsymbol{K}_{\mathbf{0}}{ }^{-1}\left(\frac{d \boldsymbol{P}_{\mathbf{0}}}{d \rho^{e}}-\frac{d \boldsymbol{K}_{\mathbf{0}}}{d \rho^{e}} \boldsymbol{U}_{\mathbf{0}}\right)+\right.\right. & \left.\left.\frac{\partial \boldsymbol{P}_{\mathbf{1}}}{\partial \rho^{e}}-\frac{d \boldsymbol{K}_{\mathbf{1}}}{d \rho^{e}} \boldsymbol{U}_{\mathbf{1}}\right]+\frac{\partial \boldsymbol{P}_{\mathbf{2}}}{\partial \rho^{e}}-\frac{d \boldsymbol{K}_{\mathbf{2}}}{d \rho^{e}} \boldsymbol{U}_{\mathbf{2}}\right\} \\
\frac{d u_{\text {saida }}}{d \rho^{e}}= & \boldsymbol{L}_{\mathbf{2}}{ }^{t} \boldsymbol{K}_{\mathbf{2}}{ }^{-1}\left(\frac{\partial \boldsymbol{P}_{\mathbf{2}}}{\partial \rho^{e}}-\frac{d \boldsymbol{K}_{\mathbf{2}}}{d \rho^{e}} \boldsymbol{U}_{\mathbf{2}}\right)+ \\
+ & \boldsymbol{L}_{\mathbf{2}}{ }^{t} \boldsymbol{K}_{\mathbf{2}}{ }^{-1} \frac{\partial \boldsymbol{P}_{\mathbf{2}}}{\partial \boldsymbol{U}_{\mathbf{1}}} \boldsymbol{K}_{\mathbf{1}}{ }^{-1}\left(\frac{\partial \boldsymbol{P}_{\mathbf{1}}}{\partial \rho^{e}}-\frac{d \boldsymbol{K}_{\mathbf{1}}}{d \boldsymbol{U}^{e}}\right)+ \\
+ & \boldsymbol{L}_{\mathbf{2}}{ }^{t} \boldsymbol{K}_{\mathbf{2}}{ }^{-1} \frac{\partial \boldsymbol{P}_{\mathbf{2}}}{\partial \boldsymbol{U}_{\mathbf{1}}} \boldsymbol{K}_{\mathbf{1}}{ }^{-1} \frac{\partial \boldsymbol{P}_{\mathbf{1}}}{\partial \boldsymbol{U}_{\mathbf{0}}} \boldsymbol{K}_{\mathbf{0}}{ }^{-1}\left(\frac{d \boldsymbol{P}_{\mathbf{0}}}{d \rho^{e}}-\frac{d \boldsymbol{K}_{\mathbf{0}}}{d \rho^{e}} \boldsymbol{U}_{\mathbf{0}}\right)
\end{aligned}
$$

A derivada do deslocamento de saída $u_{\text {saida }}$ pode ser escrita também dessa forma:

$$
\begin{aligned}
\frac{d u_{\text {saida }}}{d \rho^{e}}=\boldsymbol{\Lambda}_{\mathbf{2}}\left(\frac{\partial \boldsymbol{P}_{\mathbf{2}}}{\partial \rho^{e}}-\frac{d \boldsymbol{K}_{\mathbf{2}}}{d \rho^{e}} \boldsymbol{U}_{\mathbf{2}}\right)+ & \boldsymbol{\Lambda}_{\mathbf{1}}\left(\frac{\partial \boldsymbol{P}_{\mathbf{1}}}{\partial \rho^{e}}-\frac{d \boldsymbol{K}_{\mathbf{1}}}{d \rho^{e}} \boldsymbol{U}_{\mathbf{1}}\right)+ \\
& +\boldsymbol{\Lambda}_{\mathbf{0}}\left(\frac{d \boldsymbol{P}_{\mathbf{0}}}{d \rho^{e}}-\frac{d \boldsymbol{K}_{\mathbf{0}}}{d \rho^{e}} \boldsymbol{U}_{\mathbf{0}}\right)
\end{aligned}
$$

onde $\Lambda_{2}, \Lambda_{1}$ e $\Lambda_{0}$ são os vetores adjuntos, dados pelas equações a seguir. A forma a direita demonstra que os vetores adjuntos podem ser calculados utilizando o mesmo método numérico da resolução das análises por MEF e as mesmas matrizes $\boldsymbol{K}_{2}, \boldsymbol{K}_{1}$ e $\boldsymbol{K}_{\mathbf{0}}$ calculadas para a iteração. 


$$
\begin{array}{llll}
\boldsymbol{\Lambda}_{\mathbf{2}}=\boldsymbol{L}_{\mathbf{2}}{ }^{t} \boldsymbol{K}_{\mathbf{2}}^{-1} & \rightarrow & \boldsymbol{K}_{\mathbf{2}} \boldsymbol{\Lambda}_{\mathbf{2}}{ }^{t}=\boldsymbol{L}_{\mathbf{2}} \\
\boldsymbol{\Lambda}_{\mathbf{1}}=\boldsymbol{\Lambda}_{\mathbf{2}} \frac{\partial \boldsymbol{P}_{\mathbf{2}}}{\partial \boldsymbol{U}_{\mathbf{1}}} \boldsymbol{K}_{\mathbf{1}}{ }^{-1} & \rightarrow & \boldsymbol{K}_{\mathbf{1}} \boldsymbol{\Lambda}_{\mathbf{1}}{ }^{t}=\left(\boldsymbol{\Lambda}_{\mathbf{2}} \frac{\partial \boldsymbol{P}_{\mathbf{2}}}{\partial \boldsymbol{U}_{\mathbf{1}}}\right)^{t} \\
\boldsymbol{\Lambda}_{\mathbf{0}}=\boldsymbol{\Lambda}_{\mathbf{1}} \frac{\partial \boldsymbol{P}_{\mathbf{1}}}{\partial \boldsymbol{U}_{\mathbf{0}}} \boldsymbol{K}_{\mathbf{0}}{ }^{-1} & \rightarrow & \boldsymbol{K}_{\mathbf{0}} \boldsymbol{\Lambda}_{\mathbf{0}}{ }^{t}=\left(\boldsymbol{\Lambda}_{\mathbf{1}} \frac{\partial \boldsymbol{P}_{\mathbf{1}}}{\partial \boldsymbol{U}_{\mathbf{0}}}\right)^{t}
\end{array}
$$

Considerando que as condições de contorno de Dirichlet impostas a um dado domínio $i$ determinam derivada nula em relação a $\rho^{e}$, o cálculo de $\frac{d U_{i}(\boldsymbol{\rho})}{d \rho^{e}}$ deve ser modificado para considerar a imposição dessa derivada nula, conforme descrito no apêndice A. Assim, as equações (4.9), (4.13) e (4.17), genericamente representadas por:

$$
\boldsymbol{K}_{\boldsymbol{i}}(\boldsymbol{\rho}) \frac{d \boldsymbol{U}_{\boldsymbol{i}}(\boldsymbol{\rho})}{d \rho^{e}}=\underbrace{\frac{d \boldsymbol{P}_{\boldsymbol{i}}\left(\boldsymbol{U}_{\boldsymbol{i}-\mathbf{1}}(\boldsymbol{\rho}), \boldsymbol{\rho}\right)}{d \rho^{e}}-\frac{d \boldsymbol{K}_{\boldsymbol{i}}(\boldsymbol{\rho})}{d \rho^{e}} \boldsymbol{U}_{\boldsymbol{i}}(\boldsymbol{\rho})}_{\boldsymbol{C}_{\boldsymbol{i}}}
$$

ficam:

$$
\boldsymbol{K}_{i}^{*}(\boldsymbol{\rho}) \frac{d \boldsymbol{U}_{\boldsymbol{i}}(\boldsymbol{\rho})}{d \rho^{e}}=\boldsymbol{C}_{i}^{*}
$$

onde $\boldsymbol{K}_{i}^{*}$ é a matriz $\boldsymbol{K}_{i}$ modificada de acordo com a aplicação de condições de contorno de Dirichlet; $\boldsymbol{C}_{\boldsymbol{i}}^{*}$ é o vetor $\boldsymbol{C}_{\boldsymbol{i}}$ com valores nulos nas posições correspondentes aos graus de liberdade impostos.

Para implementar esse efeito das condições de contorno de Dirichlet no cálculo das sensibilidades pelo método adjunto, utiliza-se $\boldsymbol{K}_{0}^{*}, \boldsymbol{K}_{1}^{*}$ e $\boldsymbol{K}_{2}^{*}$ no lugar de $\boldsymbol{K}_{0}, \boldsymbol{K}_{1}$ e $\boldsymbol{K}_{2}$ no cálculo de $\boldsymbol{\Lambda}_{0}, \Lambda_{1}$ e $\boldsymbol{\Lambda}_{2}$, respectivamente. Força-se também valores nulos em $\Lambda_{0}, \Lambda_{1}$ e $\Lambda_{2}$ nas posições correspondentes aos graus de liberdade impostos. Assim, o cálculo das sensibilidades pelo método adjunto fica:

$$
\begin{array}{rlrl}
\frac{d u_{\text {saida }}}{d \rho^{e}}=\boldsymbol{\Lambda}_{\mathbf{2}}^{*}\left(\frac{\partial \boldsymbol{P}_{\mathbf{2}}}{\partial \rho^{e}}-\frac{d \boldsymbol{K}_{\mathbf{2}}}{d \rho^{e}} \boldsymbol{U}_{\mathbf{2}}\right)+ & \boldsymbol{\Lambda}_{\mathbf{1}}^{*}\left(\frac{\partial \boldsymbol{P}_{\mathbf{1}}}{\partial \rho^{e}}-\frac{d \boldsymbol{K}_{\mathbf{1}}}{d \rho^{e}} \boldsymbol{U}_{\mathbf{1}}\right)+ \\
& +\boldsymbol{\Lambda}_{\mathbf{0}}^{*}\left(\frac{d \boldsymbol{P}_{\mathbf{0}}}{d \rho^{e}}-\frac{d \boldsymbol{K}_{\mathbf{0}}}{d \rho^{e}} \boldsymbol{U}_{\mathbf{0}}\right) \\
\boldsymbol{\Lambda}_{\mathbf{2}}^{*}=\boldsymbol{L}_{\mathbf{2}}{ }^{t} \boldsymbol{K}_{\mathbf{2}}^{*-1} & \rightarrow & \boldsymbol{K}_{\mathbf{2}}^{*} \boldsymbol{\Lambda}_{\mathbf{2}}^{* t}=\boldsymbol{\Phi}_{\mathbf{2}}^{*} \\
\boldsymbol{\Lambda}_{\mathbf{1}}^{*}=\boldsymbol{\Lambda}_{\mathbf{2}}^{*} \frac{\partial \boldsymbol{P}_{\mathbf{2}}}{\partial \boldsymbol{U}_{\mathbf{1}}} \boldsymbol{K}_{\mathbf{1}}^{*-1} & \rightarrow & \boldsymbol{K}_{\mathbf{1}}^{*} \boldsymbol{\Lambda}_{\mathbf{1}}^{* t}=\boldsymbol{\Phi}_{\mathbf{1}}^{*} \\
\boldsymbol{\Lambda}_{\mathbf{0}}^{*}=\boldsymbol{\Lambda}_{\mathbf{1}}^{*} \frac{\partial \boldsymbol{P}_{\mathbf{1}}}{\partial \boldsymbol{U}_{\mathbf{0}}} \boldsymbol{K}_{\mathbf{0}}^{*-1} & \rightarrow & \boldsymbol{K}_{\mathbf{0}}^{*} \boldsymbol{\Lambda}_{\mathbf{0}}^{* t}=\boldsymbol{\Phi}_{\mathbf{0}}^{*}
\end{array}
$$


onde $\boldsymbol{\Phi}_{2}^{*}$ é uma modificação de $\boldsymbol{L}_{2} ; \boldsymbol{\Phi}_{1}^{*}$, de $\left(\boldsymbol{\Lambda}_{\mathbf{2}}^{*} \frac{\partial \boldsymbol{P}_{2}}{\partial \boldsymbol{U}_{1}}\right)^{t} ;$ e $\boldsymbol{\Phi}_{\mathbf{0}}^{*}$, de $\left(\boldsymbol{\Lambda}_{1}^{*} \frac{\partial \boldsymbol{P}_{1}}{\partial \boldsymbol{U}_{0}}\right)^{t}$. Estes vetores possuem valores nulos nas posições correspondentes aos graus de liberdade impostos.

Para que seja possível o cálculo das sensibilidades, outras derivações adicionais são necessárias.

\subsubsection{Sensibilidade - Matrizes de rigidez $K_{2}$, de condutividade térmica $K_{1}$ e de condutividade elétrica $K_{0}$}

Considerando as definições das matrizes de rigidez $\boldsymbol{K}_{\mathbf{2}}$, de condutividade térmica $\boldsymbol{K}_{1}$ e de condutividade elétrica $\boldsymbol{K}_{\mathbf{0}}$ (equações $(2.9),(2.8)$ e (2.7)):

$$
\begin{aligned}
& \boldsymbol{K}_{\mathbf{2}}(\boldsymbol{\rho})=\stackrel{M}{e=1}^{N} \boldsymbol{K}_{\mathbf{2}}^{\boldsymbol{e}}\left(\rho^{e}\right) \\
& \boldsymbol{K}_{\mathbf{1}}(\boldsymbol{\rho})=\stackrel{M}{e=1}_{e=1}^{N}\left(\boldsymbol{K}_{\mathbf{1}}^{\boldsymbol{e}}\left(\rho^{e}\right)+\boldsymbol{h}^{\boldsymbol{e}}\right) \\
& \boldsymbol{K}_{\mathbf{0}}(\boldsymbol{\rho})=\stackrel{M}{N=1}_{\boldsymbol{M}} \boldsymbol{K}_{\mathbf{0}}^{\boldsymbol{e}}\left(\rho^{e}\right)
\end{aligned}
$$

as suas derivadas em relação a $\rho^{e}$ são:

$$
\begin{aligned}
& \frac{\partial \boldsymbol{K}_{\mathbf{2}}(\boldsymbol{\rho})}{\partial \rho^{e}}=\frac{d \boldsymbol{K}_{\mathbf{2}}(\boldsymbol{\rho})}{d \rho^{e}}=\underset{e=1}{M} \frac{d \boldsymbol{K}_{\mathbf{2}}^{e}\left(\rho^{e}\right)}{d \rho^{e}} \\
& \frac{\partial \boldsymbol{K}_{\mathbf{1}}(\boldsymbol{\rho})}{\partial \rho^{e}}=\frac{d \boldsymbol{K}_{\mathbf{1}}(\boldsymbol{\rho})}{d \rho^{e}}=\underset{e=1}{M} \frac{d \boldsymbol{K}_{\mathbf{1}}^{\boldsymbol{e}}\left(\rho^{e}\right)}{d \rho^{e}} \\
& \frac{\partial \boldsymbol{K}_{\mathbf{0}}(\boldsymbol{\rho})}{\partial \rho^{e}}=\frac{d \boldsymbol{K}_{\mathbf{0}}(\boldsymbol{\rho})}{d \rho^{e}}=\underset{e=1}{M} \frac{d \boldsymbol{K}_{\mathbf{0}}^{e}\left(\rho^{e}\right)}{d \rho^{e}}
\end{aligned}
$$

onde $\boldsymbol{K}_{2}^{e}$ é a matriz de rigidez do elemento $e ; \boldsymbol{K}_{1}^{e}$ e $\boldsymbol{h}^{e}$ são as matrizes de condutividade térmica e conveç̧ão do elemento $e ; \boldsymbol{K}_{\mathbf{0}}^{e}$ é a matriz de condutividade elétrica do elemento $e$; e $N$ é o número de elementos do domínio fixo estendido.

Considerando a definição das matrizes de rigidez $\boldsymbol{K}_{2}^{e}$, de condutividade térmica $\boldsymbol{K}_{1}^{e}$ e de condutividade elétrica $\boldsymbol{K}_{\mathbf{0}}^{e}$ de um dado elemento $e$ (equações $(2.25),(2.45)$ e $(2.44))$ :

$$
\begin{aligned}
& \boldsymbol{K}_{\mathbf{2}}^{e}\left(\rho^{e}\right)=\int_{A^{e}} \boldsymbol{B}_{\mathbf{2}}^{t} \boldsymbol{D}\left(\rho^{e}\right) \boldsymbol{B}_{\mathbf{2}} d A^{e} \\
& \boldsymbol{K}_{\mathbf{1}}^{e}\left(\rho^{e}\right)=\sigma_{1}\left(\rho^{e}\right) \int_{A^{e}} \boldsymbol{B}_{\mathbf{1}}{ }^{t} \boldsymbol{B}_{\mathbf{1}} d A^{e} \\
& \boldsymbol{K}_{\mathbf{0}}^{e}\left(\rho^{e}\right)=\sigma_{0}\left(\rho^{e}\right) \int_{A^{e}} \boldsymbol{B}_{\mathbf{0}}{ }^{t} \boldsymbol{B}_{\mathbf{0}} d A^{e}
\end{aligned}
$$


as suas derivadas em relação a $\rho^{e}$ são:

$$
\begin{aligned}
& \frac{d \boldsymbol{K}_{\mathbf{2}}^{e}\left(\rho^{e}\right)}{d \rho^{e}}=\int_{A^{e}} \boldsymbol{B}_{\mathbf{2}}{ }^{t} \frac{d \boldsymbol{D}\left(\rho^{e}\right)}{d \rho^{e}} \boldsymbol{B}_{\mathbf{2}} d A^{e} \\
& \frac{d \boldsymbol{K}_{\mathbf{1}}^{e}\left(\rho^{e}\right)}{d \rho^{e}}=\frac{d \sigma_{1}\left(\rho^{e}\right)}{d \rho^{e}} \int_{A^{e}} \boldsymbol{B}_{\mathbf{1}}{ }^{t} \boldsymbol{B}_{\mathbf{1}} d A^{e} \\
& \frac{d \boldsymbol{K}_{\mathbf{0}}^{e}\left(\rho^{e}\right)}{d \rho^{e}}=\frac{d \sigma_{0}\left(\rho^{e}\right)}{d \rho^{e}} \int_{A^{e}} \boldsymbol{B}_{\mathbf{0}}{ }^{t} \boldsymbol{B}_{\mathbf{0}} d A^{e}
\end{aligned}
$$

\subsubsection{Sensibilidade - Vetor de carga estrutural $\boldsymbol{P}_{2}$}

Considerando a definição do vetor de carga estrutural (equação (2.33)), tem-se as derivadas em relação a $\rho^{e}$ e $\boldsymbol{U}_{1}$, necessárias às equações (4.23) e (4.24b):

$$
\begin{gathered}
\boldsymbol{P}_{\mathbf{2}}\left(\boldsymbol{U}_{\mathbf{1}}, \boldsymbol{\rho}\right)=-\boldsymbol{P}_{\mathbf{2 I}}+\boldsymbol{P}_{\mathbf{2 C}}=-\stackrel{M}{M=1}_{\mathbf{2} \boldsymbol{I}}^{\boldsymbol{e}}\left(\boldsymbol{U}_{\mathbf{1}}^{\boldsymbol{e}}, \rho^{e}\right)+\boldsymbol{P}_{\mathbf{2} \boldsymbol{C}}(\boldsymbol{\rho}) \\
\frac{\partial \boldsymbol{P}_{\mathbf{2}}\left(\boldsymbol{U}_{\mathbf{1}}(\boldsymbol{\rho}), \boldsymbol{\rho}\right)}{\partial \rho^{e}}=-\stackrel{M}{M}_{e=1}^{N} \frac{\partial \boldsymbol{P}_{\mathbf{2} \boldsymbol{I}}^{\boldsymbol{e}}\left(\boldsymbol{U}_{\mathbf{1}}^{\boldsymbol{e}}, \rho^{e}\right)}{\partial \rho^{e}}+\frac{\partial \boldsymbol{P}_{\mathbf{2 C}}(\boldsymbol{\rho})}{\partial \rho^{e}} \\
\frac{\partial \boldsymbol{P}_{\mathbf{2}}\left(\boldsymbol{U}_{\mathbf{1}}(\boldsymbol{\rho}), \boldsymbol{\rho}\right)}{\partial \boldsymbol{U}_{\mathbf{1}}}=-\stackrel{N}{M} \frac{\partial \boldsymbol{P}_{\mathbf{2}}^{\boldsymbol{e}}\left(\boldsymbol{U}_{\mathbf{1}}^{\boldsymbol{e}}, \rho^{e}\right)}{\partial \boldsymbol{U}_{\mathbf{1}}^{e}}
\end{gathered}
$$

Os termos não nulos de $\frac{\partial \boldsymbol{P}_{2 C}(\boldsymbol{\rho})}{\partial \rho^{e}}$ correspondem às derivadas das reações devido a condições de contorno de Dirichlet e, apesar de desconhecidos, podem ser ignorados porque serão substituídos por um valor nulo devido à aplicação das condições de contorno de Dirichlet para a derivada de $\boldsymbol{U}_{\mathbf{2}}$.

Considerando a definição de carga estrutural de um elemento e (equação (2.34)), a derivação em relação a $\rho^{e}$ fica:

$$
\begin{gathered}
\boldsymbol{P}_{\mathbf{2} \boldsymbol{I}}^{\boldsymbol{e}}\left(\boldsymbol{U}_{\mathbf{1}}^{e}, \rho^{e}\right)=\int_{A^{e}} \boldsymbol{B}_{\mathbf{2}}{ }^{t} \boldsymbol{\tau}_{\boldsymbol{I}}^{e}\left(\boldsymbol{U}_{\mathbf{1}}^{e}, \rho^{e}\right) d A^{e} \\
\frac{\partial \boldsymbol{P}_{\mathbf{2} \boldsymbol{I}}^{\boldsymbol{e}}\left(\boldsymbol{U}_{\mathbf{1}}^{e}, \rho^{e}\right)}{\partial \rho^{e}}=\int_{A^{e}} \boldsymbol{B}_{\mathbf{2}}{ }^{t} \frac{\partial \boldsymbol{\tau}_{\boldsymbol{I}}^{e}\left(\boldsymbol{U}_{\mathbf{1}}^{e}, \rho^{e}\right)}{\partial \rho^{e}} d A^{e}
\end{gathered}
$$

onde $\boldsymbol{B}_{2}$ é a matriz de operadores diferenciais das funções de forma e $\boldsymbol{\tau}_{\boldsymbol{I}}^{e}$ são as tensões iniciais do elemento $e$.

A derivada das tensões iniciais $\boldsymbol{\tau}_{\boldsymbol{I}}^{e}$ (equação (2.39)) em relação a $\rho^{e}$ é:

$$
\boldsymbol{\tau}_{\boldsymbol{I}}^{e}\left(\boldsymbol{U}_{\mathbf{1}}^{e}, \rho^{e}\right)=-\alpha\left(\rho^{e}\right) \boldsymbol{D}\left(\rho^{e}\right)\left\{\begin{array}{l}
1 \\
1 \\
0
\end{array}\right\} \boldsymbol{N}_{\boldsymbol{f}} \boldsymbol{U}_{\mathbf{1}}^{e}
$$




$$
\frac{\partial \boldsymbol{\tau}_{\boldsymbol{I}}^{e}\left(\boldsymbol{U}_{\mathbf{1}}^{\boldsymbol{e}}, \rho^{e}\right)}{\partial \rho^{e}}=-\frac{\partial \alpha\left(\rho^{e}\right)}{\partial \rho^{e}} \boldsymbol{D}\left(\rho^{e}\right)\left\{\begin{array}{l}
1 \\
1 \\
0
\end{array}\right\} \boldsymbol{N}_{\boldsymbol{f}} \boldsymbol{U}_{\mathbf{1}}^{\boldsymbol{e}}-\alpha\left(\rho^{e}\right) \frac{\partial \boldsymbol{D}\left(\rho^{e}\right)}{\partial \rho^{e}}\left\{\begin{array}{l}
1 \\
1 \\
0
\end{array}\right\} \boldsymbol{N}_{\boldsymbol{f}} \boldsymbol{U}_{\mathbf{1}}^{\boldsymbol{e}}
$$

onde $\alpha$ é o coeficiente de expansão térmica, $\boldsymbol{D}$ é a matriz de elasticidade para o estado plano de tensões e $\boldsymbol{N}_{\boldsymbol{f}}$ é a matriz das funções de forma.

A derivada de $\boldsymbol{P}_{2 \boldsymbol{I}}^{e}$ em relação a $\boldsymbol{U}_{1}^{e}$ pode ser definida como:

$$
\frac{\partial \boldsymbol{P}_{\mathbf{2 I}}^{\boldsymbol{e}}\left(\boldsymbol{U}_{\mathbf{1}}^{e}, \rho^{e}\right)}{\partial \boldsymbol{U}_{\mathbf{1}}^{e}}=\left[\frac{\partial \boldsymbol{P}_{\mathbf{2}}^{e}\left(\boldsymbol{U}_{\mathbf{1}}^{e}, \rho^{e}\right)}{\partial U_{1 k}^{e}}\right]_{k=1 \rightarrow 4}
$$

onde o resultado é uma matriz $8 \times 4$, com $k$ indicando o grau de liberdade térmico do elemento e definido por $U_{1 k}^{e}$.

Assim, a derivada de $\boldsymbol{P}_{2 \boldsymbol{I}}^{e}$ em relação a um grau de liberdade local $U_{1 k}^{e}$ é:

$$
\frac{\partial \boldsymbol{P}_{\mathbf{2} \boldsymbol{I}}^{\boldsymbol{e}}\left(\boldsymbol{U}_{\mathbf{1}}^{\boldsymbol{e}}, \rho^{e}\right)}{\partial U_{1 k}^{e}}=\int_{A^{e}} \boldsymbol{B}_{\mathbf{2}}{ }^{t} \frac{\partial \boldsymbol{\tau}_{\boldsymbol{I}}^{e}\left(\boldsymbol{U}_{\mathbf{1}}^{e}, \rho^{e}\right)}{\partial U_{1 k}^{e}} d A^{e}
$$

A derivada das tensões iniciais $\tau_{I}^{e}$ em relação a $U_{1 k}^{e}$ é:

$$
\frac{\partial \boldsymbol{\tau}_{\boldsymbol{I}}^{e}\left(\boldsymbol{U}_{\mathbf{1}}^{e}, \rho^{e}\right)}{\partial U_{1 k}^{e}}=-\alpha\left(\rho^{e}\right) \boldsymbol{D}\left(\rho^{e}\right)\left\{\begin{array}{l}
1 \\
1 \\
0
\end{array}\right\} \boldsymbol{N}_{\boldsymbol{f}} \frac{\partial \boldsymbol{U}_{\mathbf{1}}^{e}}{\partial U_{1 k}^{e}}
$$

onde $\frac{\partial \boldsymbol{U}_{1}^{e}}{\partial U_{1 k}^{e}}$ é um vetor-coluna de zeros, unitário apenas no grau de liberdade correspondente a $U_{1 k}^{e}$.

\subsubsection{Sensibilidade - Vetor de carga térmica $P_{1}$}

Considerando a definição do vetor de carga térmica (equação (2.53)), tem-se as derivadas em relação a $\rho^{e}$ e $\boldsymbol{U}_{\mathbf{0}}$, necessárias às equações $(4.23)$ e $(4.24 \mathrm{c})$ :

$$
\begin{aligned}
& \boldsymbol{P}_{\mathbf{1}}\left(U_{\mathbf{0}}, \boldsymbol{\rho}\right)=\boldsymbol{P}_{1 B}+\boldsymbol{P}_{1 C}=\stackrel{N}{\stackrel{M}{M}} \boldsymbol{P}_{1 B}^{e}\left(\boldsymbol{U}_{\mathbf{0}}^{e}, \rho^{e}\right)+\boldsymbol{P}_{1 C}(\boldsymbol{\rho}) \\
& \frac{\partial \boldsymbol{P}_{\mathbf{1}}\left(\boldsymbol{U}_{\mathbf{0}}, \boldsymbol{\rho}\right)}{\partial \rho^{e}}=\stackrel{N}{M} \frac{\partial \boldsymbol{P}_{\mathbf{1} \boldsymbol{B}}^{\boldsymbol{e}}\left(\boldsymbol{U}_{\mathbf{0}}^{e}, \rho^{e}\right)}{\partial \rho^{e}}+\frac{\partial \boldsymbol{P}_{\mathbf{1} C}(\boldsymbol{\rho})}{\partial \rho^{e}} \\
& \frac{\partial \boldsymbol{P}_{\mathbf{1}}\left(\boldsymbol{U}_{\mathbf{0}}, \boldsymbol{\rho}\right)}{\partial \boldsymbol{U}_{\mathbf{0}}}=\stackrel{N}{M} \frac{\partial \boldsymbol{P}_{\mathbf{1} \boldsymbol{B}}^{\boldsymbol{e}}\left(\boldsymbol{U}_{\mathbf{0}}^{\boldsymbol{e}}, \rho^{e}\right)}{\partial \boldsymbol{U}_{\mathbf{0}}^{\boldsymbol{e}}}
\end{aligned}
$$


Os termos não nulos de $\frac{\partial \boldsymbol{P}_{1 C}(\boldsymbol{\rho})}{\partial \rho^{e}}$ correspondem às derivadas das reações devido a condições de contorno de Dirichlet e, apesar de desconhecidos, podem ser ignorados porque serão substituídos por um valor nulo devido à aplicação das condições de contorno de Dirichlet para a derivada de $\boldsymbol{U}_{1}$, analogamente ao caso de $\frac{\partial \boldsymbol{P}_{\mathbf{2} C}(\boldsymbol{\rho})}{\partial \rho^{e}}$.

Considerando a definição do vetor de geração de calor interna da carga térmica do elemento $e$ (equação (2.54)), a derivação em relação a $\rho^{e}$ fica:

$$
\begin{gathered}
\boldsymbol{P}_{\mathbf{1 B}}^{\boldsymbol{e}}\left(\boldsymbol{U}_{\mathbf{0}}^{e}, \rho^{e}\right)=\int_{A^{e}} \boldsymbol{N}_{\boldsymbol{f}}^{t} q^{B}\left(\boldsymbol{U}_{\mathbf{0}}^{\boldsymbol{e}}, \rho^{e}\right) d A^{e} \\
\frac{\partial \boldsymbol{P}_{\mathbf{1} \boldsymbol{B}}^{\boldsymbol{e}}\left(\boldsymbol{U}_{\mathbf{0}}^{\boldsymbol{e}}, \rho^{e}\right)}{\partial \rho^{e}}=\int_{A^{e}} \boldsymbol{N}_{\boldsymbol{f}}{ }^{t} \frac{\partial q^{B}\left(\boldsymbol{U}_{\mathbf{0}}^{\boldsymbol{e}}, \rho^{e}\right)}{\partial \rho^{e}} d A^{e}
\end{gathered}
$$

onde $q^{B}\left(\boldsymbol{U}_{0}^{e}, \rho^{e}\right)$ é o termo que contém o fator gerador de calor interno na estrutura.

A derivada do fator gerador de calor interno $q^{B}$ (equação (2.56)) em relação a $\rho^{e}$ é:

$$
\begin{gathered}
q^{B}\left(\boldsymbol{U}_{\mathbf{0}}^{\boldsymbol{e}}, \rho^{e}\right)=\boldsymbol{U}_{\mathbf{0}}^{\boldsymbol{e} t} \boldsymbol{B}_{\mathbf{0}}{ }^{t} \sigma_{0}\left(\rho^{e}\right) \boldsymbol{B}_{\mathbf{0}} \boldsymbol{U}_{\mathbf{0}}^{\boldsymbol{e}} \\
\frac{\partial q^{B}\left(\boldsymbol{U}_{\mathbf{0}}^{\boldsymbol{e}}, \rho^{e}\right)}{\partial \rho^{e}}=\boldsymbol{U}_{\mathbf{0}}^{\boldsymbol{e} t} \boldsymbol{B}_{\mathbf{0}}{ }^{t} \frac{\partial \sigma_{0}\left(\rho^{e}\right)}{\partial \rho^{e}} \boldsymbol{B}_{\mathbf{0}} \boldsymbol{U}_{\mathbf{0}}^{\boldsymbol{e}}
\end{gathered}
$$

onde $\boldsymbol{U}_{\mathbf{0}}^{e}$ é o vetor que contém as tensões elétricas nodais no elemento $e$.

A derivada de $\boldsymbol{P}_{1 B}^{e}$ em relação a $\boldsymbol{U}_{\mathbf{0}}^{e}$ pode ser definida como:

$$
\frac{\partial \boldsymbol{P}_{\mathbf{1} \boldsymbol{B}}^{\boldsymbol{e}}\left(\boldsymbol{U}_{\mathbf{0}}^{e}, \rho^{e}\right)}{\partial \boldsymbol{U}_{\mathbf{0}}^{e}}=\left[\frac{\partial \boldsymbol{P}_{\mathbf{1 B}}^{\boldsymbol{e}}\left(\boldsymbol{U}_{\mathbf{0}}^{\boldsymbol{e}}, \rho^{e}\right)}{\partial U_{0 k}^{e}}\right]_{k=1 \rightarrow 4}
$$

onde o resultado é uma matriz $4 \times 4$, com $k$ indicando o grau de liberdade elétrico do elemento $e$ definido por $U_{0 k}^{e}$.

Assim, a derivada de $\boldsymbol{P}_{1 B}^{e}$ em relação a um grau de liberdade local $U_{0 k}^{e}$ é:

$$
\frac{\partial \boldsymbol{P}_{\mathbf{1} \boldsymbol{B}}^{\boldsymbol{e}}\left(\boldsymbol{U}_{\mathbf{0}}^{e}, \rho^{e}\right)}{\partial U_{0 k}^{e}}=\int_{A^{e}} \boldsymbol{N}_{\boldsymbol{f}}{ }^{t} \frac{\partial q^{B}\left(\boldsymbol{U}_{\mathbf{0}}^{\boldsymbol{e}}, \rho^{e}\right)}{\partial U_{0 k}^{e}} d A^{e}
$$

A derivada do fator gerador de calor interno $q^{B}$ em relação a $U_{0 k}^{e}$ é:

$$
\frac{\partial q^{B}\left(\boldsymbol{U}_{\mathbf{0}}^{\boldsymbol{e}}, \rho^{e}\right)}{\partial U_{0 k}^{e}}=\frac{\partial \boldsymbol{U}_{\mathbf{0}}^{\boldsymbol{e}}}{\partial U_{0 k}^{e}} \boldsymbol{B}_{\mathbf{0}}{ }^{t} \sigma_{0}\left(\rho^{e}\right) \boldsymbol{B}_{\mathbf{0}} \boldsymbol{U}_{\mathbf{0}}^{\boldsymbol{e}}+\boldsymbol{U}_{\mathbf{0}}^{\boldsymbol{e t}} \boldsymbol{B}_{\mathbf{0}}{ }^{t} \sigma_{0}\left(\rho^{e}\right) \boldsymbol{B}_{\mathbf{0}} \frac{\partial \boldsymbol{U}_{\mathbf{0}}^{e}}{\partial U_{0 k}^{e}}
$$

onde $\frac{\partial \boldsymbol{U}_{0}^{e}}{\partial U_{0 k}^{e}}$ é um vetor-coluna de zeros, unitário apenas no grau de liberdade correspondente a $U_{0 k}^{e}$. 


\subsubsection{Sensibilidade - Vetor de carga elétrica $P_{0}$}

O vetor de carga elétrica $\boldsymbol{P}_{\mathbf{0}}$ consiste apenas na corrente elétrica nodal concentrada. Os valores não nulos podem ser correntes prescritas como entrada do problema ou reações do problema elétrico à imposição de tensões (condições de contorno de Dirichlet).

Os termos não nulos de $\frac{\partial \boldsymbol{P}_{0}(\boldsymbol{\rho})}{\partial \rho^{e}}$ correspondem às derivadas das reações devido a condições de contorno de Dirichlet e, apesar de desconhecidos, podem ser ignorados porque serão substituídos por um valor nulo devido à aplicação das condições de contorno de Dirichlet para a derivada de $\boldsymbol{U}_{\mathbf{0}}$, analogamente aos casos já apresentados de $\frac{\partial \boldsymbol{P}_{2 C}(\boldsymbol{\rho})}{\partial \rho^{e}}$ e $\frac{\partial \boldsymbol{P}_{1 C}(\boldsymbol{\rho})}{\partial \rho^{e}}$.

\subsubsection{Sensibilidade - Funções de restrição de volume $G_{v l_{m}}$}

Para a implementação da Programação Linear (PL), é necessário calcular a derivada em relação a $\rho^{e}$ das funções de restrição de volume $G_{v o l_{m}}$ para uma restrição do volume de cada material $m$ (exceto o vazio) de modo específico.

Como o domínio de projeto $\Omega$ é discretizado em elementos finitos para se obter uma solução numérica do problema de otimização, considera-se a densidade relativa de um material $m$ de cada elemento $e$ (simbolizada por $\Phi_{m}^{e}$, dependente de $\rho^{e}$ ) de tal forma que o volume total de um dado material $m$ na estrutura possa ser representado pela somatória do produto entre o volume de cada elemento e a densidade relativa $\Phi_{m}^{e}\left(\rho^{e}\right)$. Assim, as funções de restrição de volume do problema são:

$$
G_{v o l_{m}}=\sum_{e=1}^{N} \Phi_{m}^{e}\left(\rho^{e}\right) V^{e} \leq \Omega_{s_{m}}
$$

onde $N$ é o numero de elementos resultantes da discretização do domínio de projeto fixo, $V^{e}$ é o volume do elemento $e ; \Omega_{s_{m}}$ é a restrição estabelecida sobre o volume total do material $m$ no domínio de projeto fixo.

É importante ressaltar que as densidades relativas $\Phi_{m}^{e}$ não são as variáveis de projeto do problema de otimização. No caso do modelo de material de função de pico, as variáveis de projeto são as pseudo-densidades $\rho^{e}$ de cada elemento. Para calcular a restrição de volume segundo a equação (4.45), deve-se usar a seguinte formulação:

$$
\begin{gathered}
\Phi_{m}^{e}\left(\rho^{e}\right)=e^{-\left(\rho^{e}-\mu_{m}\right)^{2} / 2 \sigma_{m}^{2}} \\
G_{v_{\text {ol }} m}=\sum_{e=1}^{N} e^{-\left(\rho^{e}-\mu_{m}\right)^{2} / 2 \sigma_{m}^{2}} V^{e} \leq \Omega_{s_{m}}
\end{gathered}
$$


Note que as equações (4.46) e (4.47) utilizam apenas o número de Euler (e seu expoente) do termo referente ao material $m$ do modelo de material de função de pico, descrito pela equação (4.4).

A derivada das funções $G_{v o l_{m}}$ em relação a $\rho^{e}$, dadas pela equação (4.47), fica:

$$
\begin{gathered}
G_{v o l_{m}}(\boldsymbol{\rho})=\sum_{e=1}^{N} e^{-\left(\rho^{e}-\mu_{m}\right)^{2} / 2 \sigma_{m}^{2}} V^{e} \\
\frac{\partial G_{v o l_{m}}(\boldsymbol{\rho})}{\partial \rho^{e}}=\frac{\partial\left(e^{-\left(\rho^{e}-\mu_{m}\right)^{2} / 2 \sigma_{m}^{2}}\right)}{\partial \rho^{e}} V^{e} \\
\frac{\partial G_{v o l_{m}}(\boldsymbol{\rho})}{\partial \rho^{e}}=\left(-\frac{\rho^{e}-\mu_{m}}{\sigma_{m}^{2}}\right) e^{-\left(\rho^{e}-\mu_{m}\right)^{2} / 2 \sigma_{m}^{2}} V^{e}
\end{gathered}
$$

\subsubsection{Sensibilidade - Propriedades dos materiais}

A partir da definição do modelo de interpolação de material do tipo função de pico para uma propriedade qualquer $C$ (equação (4.4)), a derivada em relação a $\rho^{e}$ é dada por:

$$
\begin{gathered}
C^{e}\left(\rho^{e}\right)=\sum_{m=1}^{M} C_{m} e^{-\left(\rho^{e}-\mu_{m}\right)^{2} / 2 \sigma_{m}^{2}}+C_{\text {vazio }} \\
\frac{\partial C^{e}}{\partial \rho^{e}}=\frac{d C^{e}}{d \rho^{e}}=\sum_{m=1}^{M} C_{m}\left(-\frac{\rho^{e}-\mu_{m}}{\sigma_{m}^{2}}\right) e^{-\left(\rho^{e}-\mu_{m}\right)^{2} / 2 \sigma_{m}^{2}}
\end{gathered}
$$

A partir da definição do modelo de interpolação de material do tipo função de pico, as derivadas em relação a $\rho^{e}$ das propriedades dos materiais são obtidas substituindo-se os valores das propriedades de cada material em $C_{m}$ na equação (4.49). As propriedades dos materiais interpoladas são: cada componente da matriz de elasticidade $\boldsymbol{D}$ (calculada a partir das propriedades de cada material), coeficiente de expansão térmica $\alpha$, condutividade elétrica $\sigma_{0}$ ou condutividade térmica $\sigma_{1}$. 


\section{Implementação Numérica do MOT}

Neste capítulo são apresentados os diferentes algoritmos para se resolver o problema de otimização estrutural (seção 5.1). Entre elas, o método de Programação Linear Sequencial (PLS) foi o escolhido por razões expostas na seção 5.1.1. Uma visão geral e detalhes da implementação da rotina são dadas na seção 5.2 e o filtro implementado é descrito na seção 5.3 .

\subsection{Técnicas de solução do problema de otimização}

Entre os principais métodos para solução de problemas de otimização estão os métodos analíticos e os métodos numéricos. Entre os métodos analíticos existentes estão o cálculo diferencial e o cálculo variacional. Os métodos analíticos permitem, em geral, somente a solução de problemas simples de otimização, como por exemplo, estruturas de geometria simples, como uma viga ou treliças. Ao contrário do analítico, os métodos numéricos permitem trabalhar com problemas complexos. Estes últimos podem ser classificados em dois tipos: específicos e gerais. Entre os métodos específicos, pode-se citar o critério de optimalidade, que consiste num método numérico de base empírica para a solução de problemas de otimização (uma revisão sobre este método pode ser encontrada no trabalho de Hassani e Hinton (1998)). Em geral, uma formulação específica deve ser desenvolvida para cada problema, o que faz com que o critério de optimalidade tenha aplicação restrita a alguns problemas de otimização estrutural. No entanto, são mais eficientes computacionalmente do que os métodos numéricos genéricos, fato comprovado por vários problemas de otimização topológica, entre eles o problema de minimização de flexibilidade média (ou maximização de rigidez) com restrição no volume de material (SUZUKI; KIKUCHI, 1991; SIGMUND, 2001a); o problema de otimização de estruturas discretas, tipo treliças (ZHOU; ROZVANY, 1991); o problema de maximização 
da freqüência de ressonância (MA; KIKUCHI; CHENG, 1995), e recentemente no projeto de mecanismos flexíveis (ANANTHASURESH; KOTA; GIANCHANDANI, 1994; LIMA, 2002; BENDSØE; SIGMUND, 2002). Os métodos genéricos podem ser aplicados a qualquer problema de otimização, estrutural ou não. Eles podem ser de dois tipos: métodos de programação matemática e métodos probabilísticos (HAFTKA; GURDAL; KAMAT, 1990). Os métodos de programação matemática são classificados em métodos de programação linear, programação não-linear e métodos baseados em teoria de aproximações como Programação Linear Sequencial (PLS), Programação Quadrática Sequencial (PQS) e o "Method of Moving Asymptotes" (MMA) (SVANBERG, 1987). A diferença essencial dos métodos de programação matemática em comparação com os métodos probabilísticos é que os últimos procuram encontrar o mínimo global do problema de otimização evitando os mínimos locais. Já os métodos de programação matemática fornecem um mínimo local (ao menos que o problema somente possua um único mínimo, que nesse caso será o mínimo global). Os métodos probabilísticos apesar de buscar o mínimo global, não são muito práticos ao trabalhar com um grande número de variáveis de projeto, já que a população e o número de análises para esses problemas são enormes (ROZVANY, 2001).

O problema de otimização no projeto de micromecanismos eletrotermomecânicos e, em geral, no projeto de sistemas modelados em domínios multivariáveis é, basicamente, um problema não-linear, o qual freqüentemente requer várias restrições e vários milhares de variáveis de projeto. Portanto, algoritmos eficientes de programação matemática devem ser aplicados para resolver este tipo problema. Assim, com esses requisitos em mente, a PLS é o método escolhido neste trabalho para a solução do problema de otimização mostrado na equação (4.1), principalmente considerando que este método vem sendo aplicado com sucesso na otimização topológica (SIGMUND, 1996; NISHIWAKI et al., 1998; SILVA et al., 1999; SILVA; NISHIWAKI; KIKUCHI, 2000). Da PLS, pode-se destacar também que, por usar apenas a primeira derivada (ao contrário do PQS, por exemplo), tem um custo computacional relativamente baixo, além de ser facilmente implementado por permitir o uso de rotinas disponíveis na Internet.

\subsubsection{Programação Linear Sequencial (PLS)}

A PLS pode ser definida como uma rotina que resolve, seqüencialmente, vários subproblemas lineares na busca da solução de um problema não-linear, o 


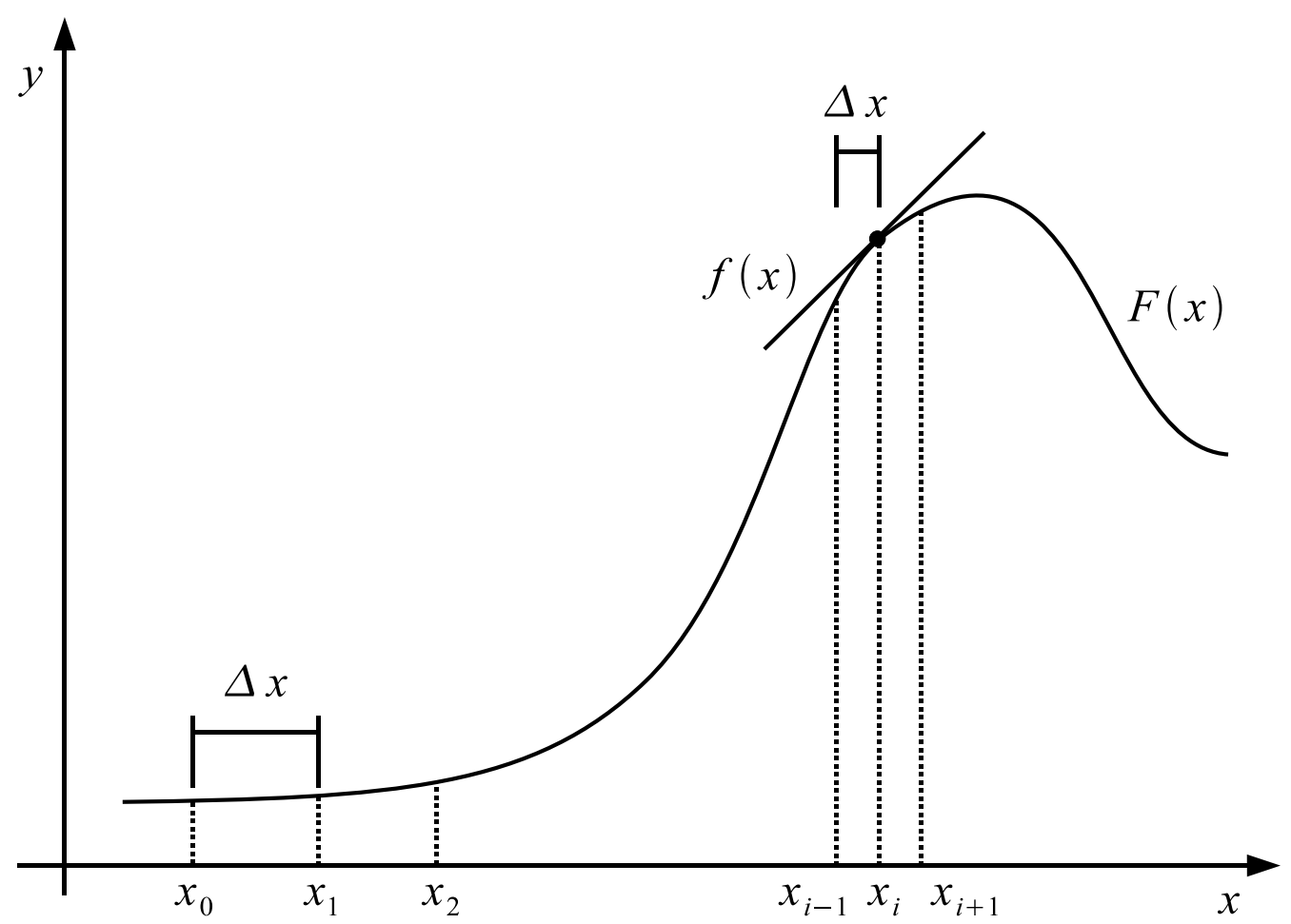

Figura 5.1: Programação Linear Sequencial e os limites móveis.

qual geralmente possui uma função objetivo complexa, um grande número de variáveis de projeto e várias restrições (HAFTKA; GURDAL; KAMAT, 1990). Essa rotina é de fácil implementação, ao permitir trabalhar com rotinas simples e comuns de Programação Linear (PL), como o SIMPLEX (DANTZIG, 1963) ou Kamarkar (HAFTKA; GURDAL; KAMAT, 1990), que estão largamente disponibilizadas gratuitamente na Internet. Na PLS, a linearidade é obtida aproximando-se a função objetivo e restrições por séries de Taylor e limitando a variação do valor de cada variável de projeto em cada subproblema linear através dos chamados limites móveis para que a aproximação linear seja válida (THOMAS; VANDERPLAATS; SHYY, 1992).

Para entender melhor o conceito da PLS e dos limiteis móveis, considere a função não-linear $F(x)$ mostrada no gráfico da figura 5.1.

Considere também a função linear $f(x)$ como uma aproximação da função não-linear $F(x)$ em torno do ponto $x_{i}$. Observa-se que a aproximação linear $f(x)$ somente é válida em torno do ponto $x_{i}$, distanciando-se muito do valor real da função $F(x)$ a medida que se afasta de $x_{i}$. Desta maneira, definem-se os valores máximo e mínimo em torno de $x_{i}$, onde a aproximação linear é válida, através de limites móveis da variável $x_{i}$. A cada iteração, a rotina de PLS maximiza a função linear $f(x)$ no intervalo dos limites móveis compreendidos entre $x_{i}^{\text {min }}$ e $x_{i}^{\text {max }}$ e encontra o valor ótimo para a variável $x_{i}$ que será utilizada na iteração 
seguinte. Assim as iterações seguem sucessivamente, substituindo-se como valor inicial da iteração posterior o valor ótimo de $x_{i}$ encontrado na iteração anterior, até ocorrer a convergência da solução. Os limites da variável $x_{i}$ são considerados móveis pelo fato de assumirem um valor diferente a cada iteração da rotina de PLS. Esses limites são definidos com base em valores relativos a uma grandeza representativa das variáveis de projeto. No presente trabalho, a distância entre o valor da variável $x_{i}$ e seus respectivos limites móveis é dada por uma porcentagem do maior desvio padrão das funções de pico para a dada iteração do processo de otimização.

Na PLS, um problema comum é a definição dos limites móveis. Se forem muito grandes, o erro da aproximação também será grande, podendo causar inclusive a perda do ponto ótimo. Se forem muito pequenos, o custo computacional para obter a solução será muito alto. Uma possibilidade para tentar minimizar esse impasse e tornar mais rápida a convergência da solução é assumir valores grandes para os limites móveis na região em que o valor da segunda derivada (curvatura) da função não-linear $F(x)$ seja pequeno (região do ponto $x_{0}$ mostrada na figura 5.1), pois nessa região a função não-linear $F(x)$ tem praticamente o mesmo comportamento da aproximação linear $f(x)$. Já na região em que o valor da segunda derivada da função $F(x)$ seja grande, os limites móveis devem ser pequenos. Portanto, a medida em que se aproxima da convergência da solução (ponto de máximo da função não-linear $F(x)$ ), deve-se reduzir bastante os valores dos limites móveis, caso contrário pode-se ter um intervalo entre $x_{i}^{\text {min }}$ e $x_{i}^{\max }$ grande o suficiente para nunca achar o ponto de máximo, causando uma oscilação nos valores de sucessivos $x_{i}$ em torno do valor ótimo.

\section{Programação Linear (PL) na PLS}

Como foi mencionado anteriormente, o problema de otimização não-linear deve ser aproximado utilizando sucessivos subproblemas de otimização linear. Assim, o método de Programação Linear (PL) se destina à solução dos problemas de otimização linear, ou seja, problemas em que a função objetivo e as restrições são funções lineares em relação às variáveis de projeto (HAFTKA; GURDAL; KAMAT, 1990), matematicamente expresso como: 


$$
\begin{array}{rlrl}
\max _{\boldsymbol{x}} \quad f(x) & =a_{1} x_{1}+a_{2} x_{2}+\ldots+a_{N} x_{N} & \\
\text { tal que } g_{i}(x) & =b_{1} x_{1}+b_{2} x_{2}+\ldots+b_{N} x_{N} \leq c_{i}, \quad\left(i=1, \ldots, m_{1}\right) \\
g_{j}(x) & =d_{1} x_{1}+d_{2} x_{2}+\ldots+d_{N} x_{N} \leq e_{j}, \quad\left(j=1, \ldots, m_{2}\right) \\
g_{k}(x) & =h_{1} x_{1}+h_{2} x_{2}+\ldots+h_{N} x_{N} \leq l_{k}, \quad\left(k=1, \ldots, m_{3}\right)
\end{array}
$$

Aplicando as idéias anteriores ao problema de otimização não-linear (em relação às densidades de cada elemento finito) para o projeto de micromecanismos eletrotermomecânicos, expresso na equação (4.1), deve-se linearizar esse problema calculando a série de Taylor de primeiro grau da função objetivo (HAFTKA; GURDAL; KAMAT, 1990). Sabe-se do cálculo diferencial que a série de Taylor pode representar uma função $F(x)$ para $x$ próximo de um valor $x_{0}$ a menos de um erro $e(x)$, como ilustra a equação abaixo:

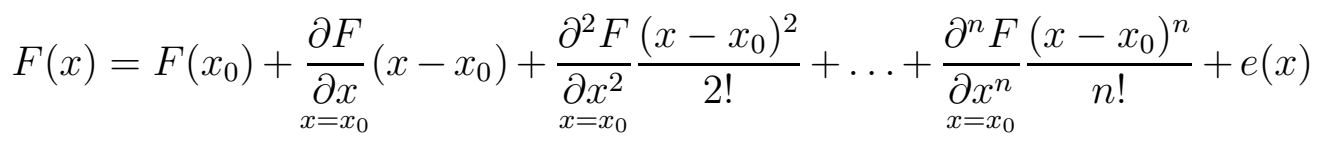

Para uma função multivariável, sendo $N$ o número de variáveis, tem-se:

$$
\begin{aligned}
& F\left(x_{1}, x_{2}, \ldots, x_{N}\right)=F\left(x_{10}, x_{20}, \ldots, x_{N 0}\right)+ \\
& +\frac{\partial F}{\partial x_{1}}\left(x_{1}-x_{10}\right)+\ldots+\frac{\partial^{n} F}{\partial x_{1}^{n}} \frac{\left(x_{1}-x_{10}\right)^{n}}{n !}+ \\
& +\frac{\partial F}{\partial x_{2}}\left(x_{2}-x_{20}\right)+\ldots+\frac{\partial^{n} F}{\partial x_{2}^{n}} \frac{\left(x_{2}-x_{20}\right)^{n}}{n !}+ \\
& +\frac{\partial F}{\partial x_{N}}\left(x_{N}-x_{N_{0}}\right)+\ldots+\frac{\partial^{n} F}{\partial x_{N}^{n}} \frac{\left(x_{N}-x_{N_{0}}\right)^{n}}{n !}+ \\
& +e\left(x_{1}, x_{2}, \ldots, x_{N}\right)
\end{aligned}
$$

Os termos com derivada de segunda ordem ou superior são desprezados, pois o que interessa é a representação linear da função objetivo para ser utilizada na PL. No entanto, outras aproximações (quadrática, etc.) também podem ser adotadas, considerando-se mais termos na série de Taylor. Nesse caso, o custo computacional aumentaria para obter as informações desses gradientes (derivadas) de maior ordem. Portanto, neste trabalho a função objetivo $F_{O b j}$ 
é linearizada pela seguinte função de primeiro grau:

$$
\begin{array}{r}
F_{O b j}^{\text {linear }}(\boldsymbol{\rho})=F_{O b j}\left(\boldsymbol{\rho}_{\mathbf{0}}\right)+\frac{\partial F_{O b j}}{\partial \rho^{e=1}}\left(\rho^{e=1}-\rho_{0}^{e=1}\right)+\frac{\partial F_{O b j}}{\partial \rho_{\mathbf{0}}^{e=2}}\left(\rho^{e=2}-\rho_{0}^{e=2}\right)+ \\
\boldsymbol{\rho = \boldsymbol { \rho } _ { \mathbf { 0 } }} \\
+\ldots+\frac{\partial F_{O b j}}{\partial \rho^{e=N}}\left(\rho^{e=N}-\rho_{0}^{e=N}\right) \\
\boldsymbol{\rho}=\boldsymbol{\rho}_{\mathbf{0}}
\end{array}
$$

onde a função objetivo $F_{O b j}$ pode representar tanto $F_{E T M}$ (equação $(4.1)$ ), como $F_{E T M_{\text {multi }}}$ (equação (4.2)), as funções objetivo usadas respectivamente para o projeto de micromecanismos sem e com a consideração de multiflexibilidade; $\boldsymbol{\rho}_{0}$ é o ponto em torno do qual a linearização é realizada.

Separando os termos constantes dos termos dependentes das variáveis de projeto (elementos do vetor $\boldsymbol{\rho}$ ), tem-se:

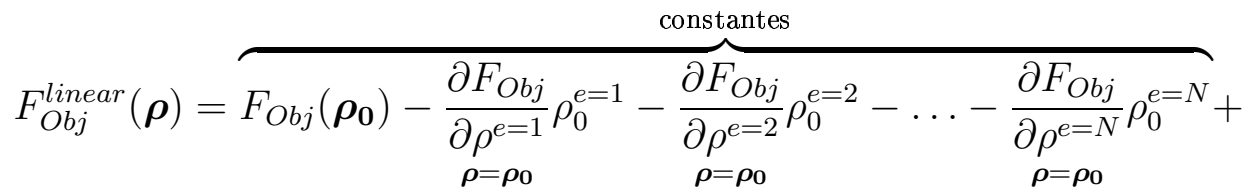

$$
\begin{aligned}
& +\frac{\partial F_{O b j}}{\partial \rho^{e=1}} \rho^{e=1}+\frac{\partial F_{O b j}}{\partial \rho^{e=2}} \rho^{e=2}+\ldots+\frac{\partial F_{O b j}}{\partial \rho^{e=N}} \rho^{e=N}
\end{aligned}
$$

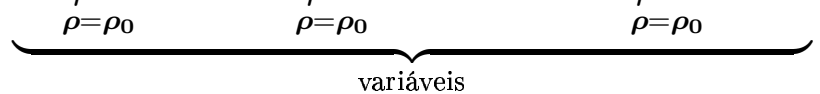

As constantes podem ser retiradas da equação (5.5), já que não influenciam no processo de maximização da função (HAFTKA; GURDAL; KAMAT, 1990). Desta maneira a função a ser maximizada na PL é dada por:

$$
F_{O b j}^{\text {linear }}(\boldsymbol{\rho})=\frac{\partial F_{O b j}}{\partial \rho^{e=1}} \rho^{e=1}+\frac{\partial F_{O b j}}{\partial \rho^{e=2}} \rho^{e=2}+\ldots+\frac{\partial F_{O b j}}{\partial \rho_{\mathbf{0}}=\boldsymbol{\rho}_{\mathbf{0}}} \rho^{e=N} \rho^{e=N}
$$

Da equação (5.6), observa-se que a função a ser maximizada é linear em relação às variáveis de projeto. Os coeficientes das densidades são as derivadas (ou sensibilidades do problema de otimização) da função objetivo do problema de otimização $F_{O b j}$ (que pode ser tanto $F_{E T M}$, como $F_{E T M_{m u l t i}}$ ) em relação a cada variável do projeto.

A PL exige que as restrições do problema de otimização sejam funções lineares em relação às variáveis de problema. As funções das restrições de volume por material $G_{v o l_{m}}$ podem ser linearizadas da mesma maneira que a função objetivo do problema de otimização $F_{O b j}$, mas sem desprezar a parte constante da série 
de Taylor:

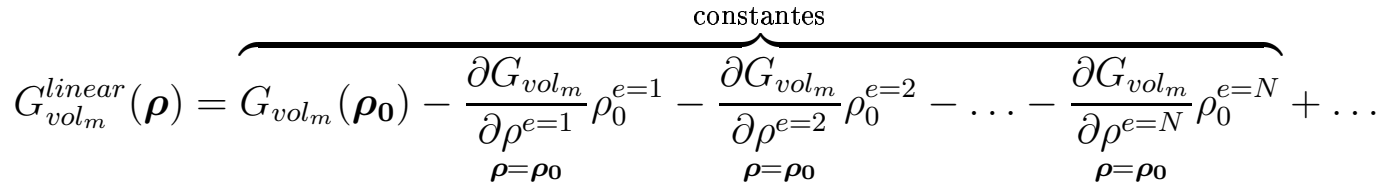

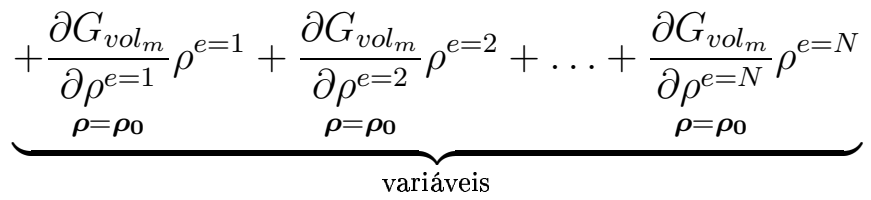

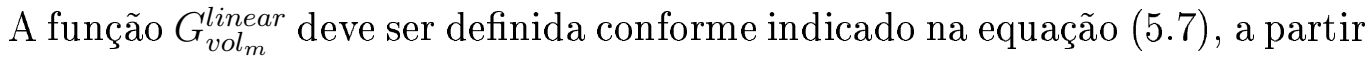
das derivadas parciais de $G_{v_{0} l_{m}}$ em relação a todas as densidades $\rho^{e}$ (equação (4.48)) do domínio fixo estendido.

Outro fator importante na implementação numérica do problema de otimização não-linear é evitar singularidades na solução das equações do MEF expostas no capítulo 2. Caso as matrizes locais de condutividade elétrica, térmica e de rigidez $\left(\boldsymbol{K}_{0}^{e}, \boldsymbol{K}_{1}^{e}\right.$ e $\boldsymbol{K}_{2}^{e}$, respectivamente) se tornem matrizes nulas (ver equações (2.25), (2.26), (2.44) e (2.45)), as matrizes globais de condutividade elétrica, térmica e de rigidez poderiam ter termos nulos na diagonal, o que gera divisões por zero durante as operações realizadas com essas matrizes. Portanto, mesmo que o valor das propriedades relacionadas a $\rho^{e}$ sejam nulas (representadas no MEF global por $\boldsymbol{\rho}$ ), os tensores das propriedades de cada elemento não deve resultar numa matriz nula. Isso pode ser conseguido definindo os tensores das propriedades do vazio $\left(\boldsymbol{C}_{\boldsymbol{v a z i o}}^{\boldsymbol{e}}\right)$ como sendo muito pequenos (próximo de zero) mas não nulos (ver equações (4.3) e (4.4)).

Assim, o subproblema linear a ser solucionado pela PLS, uma vez discretizado o domínio fixo de projeto, é:

$$
\begin{aligned}
& \max _{\boldsymbol{\rho}} F_{O b j}^{\text {linear }}(\boldsymbol{\rho})=\frac{\partial F_{O b j}}{\partial \rho^{e=1}} \rho^{e=1}+\frac{\partial F_{O b j}}{\partial \rho^{e=2}} \rho^{e=2}+\ldots+\frac{\partial F_{0 b j}}{\partial \rho_{0}} \rho^{e=N} \rho^{e=N} \\
& \text { tal que } G_{\text {volm }}^{\text {linear }} \leq \Omega_{s_{m}} \quad(m=1, \ldots, M) \quad \text { (restrições de volume - material } m \text { ) } \\
& K_{0}(\rho) U_{0}(\rho)=P_{0} \\
& \text { (equação (2.4)) } \\
& K_{1}(\rho) U_{1}(\rho)=P_{1}\left(U_{0}(\rho), \rho\right) \\
& \text { (equação (2.5)) } \\
& K_{2}(\rho) U_{2}(\rho)=P_{2}\left(U_{1}(\rho), \rho\right)
\end{aligned}
$$


A solução numérica do problema de PL, equação (5.8), pode ser obtida usando-se o método SIMPLEX (DANTZIG, 1963; PRESS et al., 1999) ou o método Kamarkar (HAFTKA; GURDAL; KAMAT, 1990) que buscam, dentro de um conjunto de soluções possíveis para o problema de PL, a solução que satisfaz as restrições e maximiza a função objetivo.

\subsection{Implementação do MOT}

Neste trabalho, foi implementado um "software" (programa de computador) que resolve o problema de otimização através de rotinas de MEF e PL. As rotinas foram escritas usando a linguagem C. Neste trabalho, foi utilizada a rotina SIMPLEX DSPLP (HANSON; HIEBERT, 1992). A DSPLP, de autoria de Hanson, R. J. e Hiebert, K. L. (Sandia National Laboratories), é uma rotina de programação linear que resolve problemas com até alguns milhares de variáveis e restrições. Essa rotina faz parte da biblioteca SLATEC, composta de rotinas de emprego geral para estatística e matemática e escritas em Fortran 77.

O modelo de material implementado foi o de função de pico, com a pseudodensidade de um dado elemento $e$ representada por uma variável de projeto $\rho^{e}$.

Os dados de entrada são fornecidos ao "software" por meio de arquivos com informações sobre a geometria, coordenadas dos nós, conectividade dos elementos, condições de contorno e aplicação das cargas no domínio inicial (domínio fixo estendido de projeto) para cada caso de carga, as propriedades físicas dos materiais a serem distribuído, rigidez da peça atuada e um vetor com a densidade inicial de cada elemento (valores iniciais para a PL). Os arquivos também contém dados das funções de pico (média, desvios padrão e continuação dos desvios padrão), das restrições de volume (valor inicial, final e continuação) e os demais parâmetros de otimização relativos ao número de iterações, filtro espacial e função objetivo. Com os dados necessários, inicia-se o "loop" de otimização, cujas iterações são limitadas por um número máximo. Um fluxograma do método pode ser visto na figura 5.2.

Os módulos principais do MOT são o módulo de análise e o módulo de otimização. O módulo de análise é usado para calcular a resposta da estrutura, que nesse caso é o deslocamento de um ponto específico da estrutura, sendo também usado no cálculo das sensibilidades. Esta análise corresponde a calcular a variação na resposta estrutural devido a uma pequena variação nas variáveis de projeto, como as pseudo-densidades do material em cada elemento obtido da 


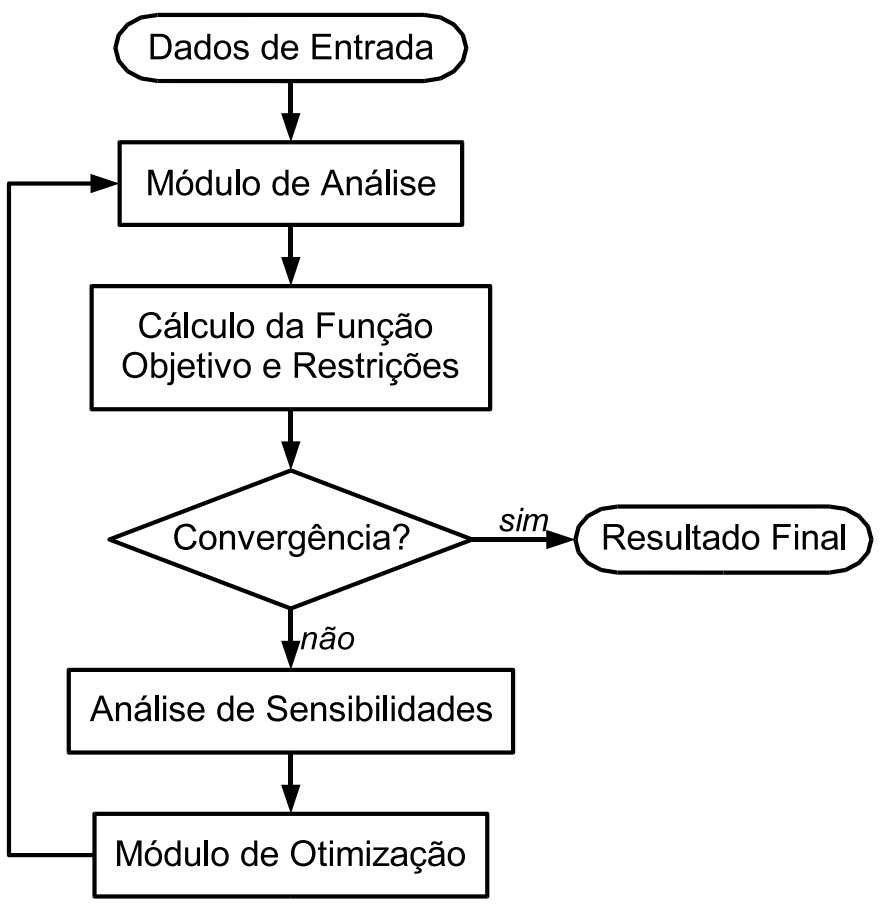

Figura 5.2: Fluxograma do Método de Otimização Topológica.

discretização do domínio inicial. Baseado na análise de sensibilidade, o módulo de otimização calcula os novos valores das variáveis de projeto que melhoram a função objetivo. Geralmente, é necessário um processo iterativo do módulo de análise, seguido da análise de sensibilidade e do módulo de otimização, para encontrar a estrutura ótima. A cada vez que o módulo de análise é executado, calcula-se o valor da função objetivo e também das funções de restrição.

Uma vez iniciado o ciclo de otimização, e através da rotina de MEF, são resolvidos seqüencialmente, os três subproblemas em questão: problema elétrico, eletrotérmico e termoelástico. O resultado de cada uma das análises é a distribuição das tensões elétricas, das variações de temperatura e dos deslocamentos, respectivamente, em cada nó do problema discretizado. As matrizes dos subproblemas de cada elemento (equações $(2.25),(2.44)$ e (2.45)) são calculadas através de uma rotina de integração numérica de Gauss-Legendre $(2 \times 2$ pontos). A rotina de $\mathrm{MEF}$, para cada subproblema, calcula as matrizes globais de condutividade elétrica, térmica e de rigidez de acordo com a conectividade de cada elemento da malha.

Com as matrizes de cada subproblema obtidas, a rotina de MEF armazena a respectiva matriz global no formato esparso, de acordo com Press et al. (1999), e resolve o sistema de equações de equilíbrio de cada subproblema através de uma rotina de Gradientes Conjugados (válida somente para matrizes positivo-definidas). As tensões elétricas resultantes do subproblema elétrico são 


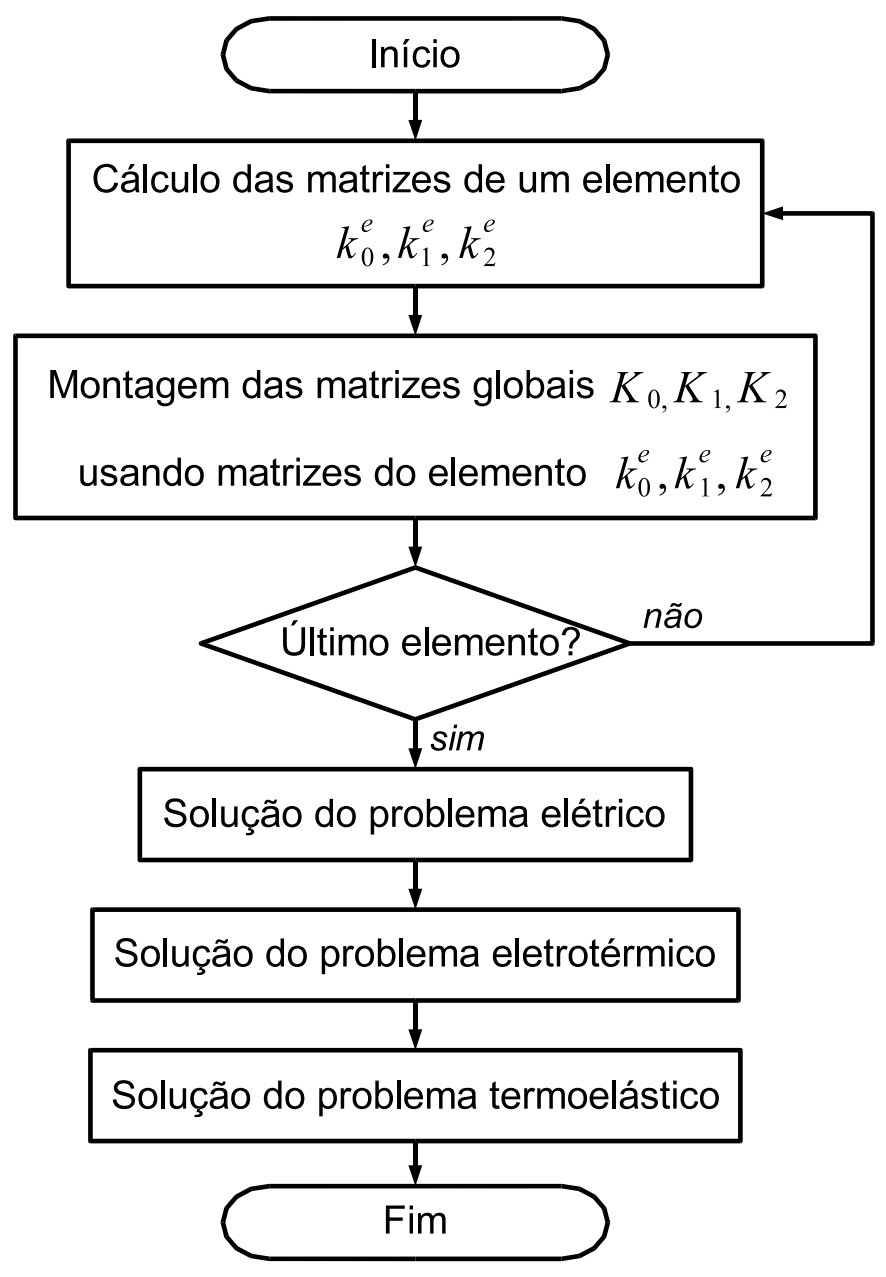

Figura 5.3: Diagrama de fluxo para os subproblemas de MEF.

utilizadas como dados de entrada no subproblema eletrotérmico, e as variações de temperatura obtidas neste problema são os dados de entrada para o subproblema termoelástico. Este último problema fornece os deslocamentos nodais. A figura 5.3 mostra o fluxograma específico da rotina de MEF.

A seguir, é calculada a função objetivo e a restrição de volume (linearizada para ser usada na PL) do problema de otimização. Antes de entrar na rotina de otimização das variáveis de projeto, determinam-se os limites móveis da PLS da densidade de cada elemento $e$. Inicialmente é atribuído aos limites móveis um passo de uma certa porcentagem (7,5\%, neste caso) em relação ao maior desvio padrão utilizado no modelo de interpolação de material. Ou seja, em cada iteração e para cada elemento $e$, o seu limite móvel superior $\rho_{\max }^{e}$ é igual ao valor de densidade mais um passo e o seu limite móvel inferior $\rho_{\min }^{e}$ é igual ao valor da densidade menos um passo. À medida que as iterações se aproximam da convergência da função objetivo, o passo dos limites móveis é diminuído sucessivamente. Isto é feito comparando-se os valores da função objetivo de três iterações consecutivas (iteração atual, uma anterior e duas anteriores). 
Lembrando-se que $F_{O b j}$ pode representar tanto $F_{E T M}$, como $F_{E T M_{m u l t i}}$, se a diferença entre o valor da função objetivo atual $F_{O b j}\left(\boldsymbol{\rho}_{\boldsymbol{i}}\right)$ e de uma iteração anterior $F_{O b j}\left(\boldsymbol{\rho}_{\boldsymbol{i}-1}\right)$ estiver entre $30 \%$ e $60 \%$ da diferença entre o valor da função objetivo de uma iteração anterior $F_{O b j}\left(\boldsymbol{\rho}_{\boldsymbol{i}-\mathbf{1}}\right)$ e de duas anteriores $F_{O b j}\left(\boldsymbol{\rho}_{\boldsymbol{i - 2}}\right)$, o passo dos limites móveis é reduzido (para $5 \%$ ). Se $F_{O b j}\left(\boldsymbol{\rho}_{\boldsymbol{i - 1}}\right)$ for menor que $30 \%$ de $F_{O b j}\left(\boldsymbol{\rho}_{\boldsymbol{i - 2}}\right)$, reduz-se o passo dos limites móveis para uma porcentagem ainda menor do maior desvio padrão $(2,5 \%)$.

A próxima etapa consiste na otimização das variáveis de projeto através do algoritmo DSPLP, que necessita da informação da sensibilidade do meio, ou seja, da derivada da função objetivo em relação às variáveis de projeto (pseudo-densidades de cada elemento finito), além da linearização da restrição de volume. Na primeira iteração, o DSPLP utiliza o vetor de distribuição de densidades iniciais dos elementos, que, por simplicidade, pode conter valores de densidades iguais para todos os elementos, ou mesmo densidades aleatórias para cada elemento, que servem de ponto de partida para o processo de busca da distribuição ótima de pseudo-densidades dos elementos. Logo, o algoritmo de otimização fornece uma nova distribuição de material, que é atualizada no modelo de MEF e segue-se uma nova iteração até alcançar a convergência.

Ao final de cada iteração, os desvios padrão da função de pico são reduzidos no que também é conhecido como continuação, conforme já discutido na seção 4.2.2. Este processo é realizado da mesma forma que em (YIN; ANANTHASURESH, 2001):

$$
\sigma_{m_{i+1}}^{2}=\sigma_{m_{i}}^{2}\left(1-\omega_{D P_{m}}\right)
$$

onde $\sigma_{m}$ é o desvio padrão para o pico determinado pelo termo referente ao material $m ; i$ é o número da iteração atual; e $\omega_{D P_{m}}$ é o coeficiente de continuação do desvio padrão de cada material $m$.

De forma semelhante, o volume máximo $\Omega_{s_{m}}$ de cada material $m$ se inicia com um valor mais alto, reduzindo-se de modo a se aproximar assintoticamente do valor final desejado:

$$
\Omega_{s_{m i+1}}=\Omega_{s_{m i}}-\left(\Omega_{s_{m i}}-\Omega_{s_{m f i n a l}}\right) \omega_{R V_{m}}
$$

onde $i$ é o número da iteração atual; $\Omega_{s_{m f i n a l}}$ é o valor final do volume máximo de cada material $m$ e $\omega_{R V_{m}}$ é o coeficiente de continuação do volume máximo do material $m$. 


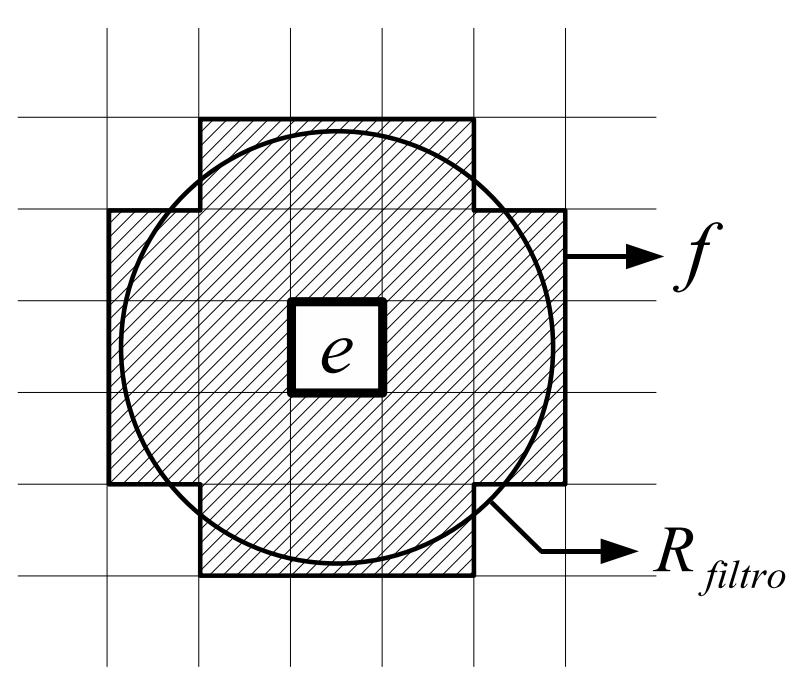

Figura 5.4: Raio de ação do filtro espacial - elemento $e$ influenciado pelos elementos $f$.

\subsection{Filtro Implementado}

Para contornar os problemas de dependência de malha e instabilidade de tabuleiro (descritos na seção 3.4), foi implementado o filtro espacial de Cardoso e Fonseca (1999), já abordado na seção 3.4.4. Na rotina implementada, o filtro foi aplicado sobre os limites móveis.

O filtro espacial de Cardoso e Fonseca (1999) implementado considera os limites móveis das variáveis de projeto relacionadas aos elementos $f$, que estão dentro do raio de ação $R_{\text {filtro }}$, para atualizar os limites móveis da variável de projeto de um dado elemento $e$. Para um elemento $f$ estar dentro do raio de ação do filtro, a distância entre os centróides dos elementos e e $f$ deve ser menor que $R_{\text {filtro }}$ (ver figura 5.4).

Assim, as atualizações dos limites inferior e superior de cada variável de projeto $\rho^{e}$, representados por $\rho_{\text {min }}^{e}$ e $\rho_{\text {max }}^{e}$, de um dado elemento $e$ são feitas pela técnica de filtragem do seguinte modo:

$$
\begin{aligned}
\rho_{\text {lim }}^{e} & =\frac{\rho_{\text {lim }}^{e} V^{e}+\bar{w} \sum^{n f} \rho_{\text {lim }}^{f} V^{f}}{V^{e}+\bar{w} \sum^{n f} V^{f}} \\
\bar{w} & =\frac{\sum^{n f} w^{f}}{n f} \\
w^{f} & =\frac{R_{\text {filtro }}-R^{f}}{R_{\text {filtro }}}
\end{aligned}
$$

onde $\rho_{\text {lim }}^{e}$ e $\rho_{\text {lim }}^{f}$ são um limite móvel (inferior ou superior) das variáveis de projeto $\rho^{e}$ e $\rho^{f}$, respectivamente; $V^{e}$ e $V^{f}$ são o volume dos elementos e e $f ; n f$ é o 
número de elementos $f$ considerados pelo filtro; $R^{f}$ é a distância medida entre os centróides dos elementos e e $f ; R_{\text {filtro }}$ é o raio de ação do filtro.

Nota-se que o único parâmetro que regula a funcionalidade deste filtro é o raio de ação $R_{\text {filtro }}$. 


\section{$6 \quad$ Resultados}

Neste capítulo, os resultados obtidos para o problema de otimização descrito são apresentados. Primeiramente, procura-se reproduzir um resultado obtido por Yin e Ananthasuresh (2002) com o uso de dois materiais e obter um mecanismo de mesma funcionalidade composto por três materiais. Em seguida, será mostrado um estudo do efeito de alguns parâmetros na otimização, como rigidez da peça atuada, uso de restrições de volume, uso do filtro espacial, coeficiente de continuação dos desvios padrão e parâmetros da função de pico (média e desvios padrão). Considerando multiflexibilidade, alguns resultados foram obtidos para otimizações com um e dois materiais. Também foram projetados mecanismos atuados por fluxo de calor, onde determinada porção deste mecanismo é aquecida, por exemplo, com a incidência de um feixe de "laser". Os resultados foram obtidos num microcomputador Pentium 4 HT $3.2 \mathrm{GHz}$, com 512Mb de memória RAM.

\subsection{Atuador de movimento inverso}

No mecanismo do atuador de movimento inverso, o objetivo é fazer com que o atuador puxe uma carga ao invés de empurrar, que seria o movimento normal de um mecanismo cuja estrutura se expande durante a atuação. Um atuador desse tipo foi obtido por Yin e Ananthasuresh (2002). De modo a reproduzir o resultado, foi utilizado um domínio fixo estendido semelhante. Assim, o domínio tem dimensões $3 \mathrm{~mm} \times 2 \mathrm{~mm}$, discretizado em $30 \times 20$ elementos, e espessura de $15 \times 10^{-3} \mathrm{~mm}$. Nas quatro extremidades, foram aplicadas restrições em $x$ e $y \mathrm{e}$ temperatura ambiente $T_{a m b}$. Entre as duas extremidades inferiores, foi aplicada uma tensão elétrica $V$ de $30 \mathrm{~V}$. Sobre toda a superfície superior, foi considerado um coeficiente de convecção $h_{0}=2,5 \times 10^{-4} \mathrm{~W} / \mathrm{mm}^{2} \cdot \mathrm{K}$. Deseja-se maximizar o deslocamento $u_{\text {saida }}$, onde uma peça de rigidez $K=2 \mathrm{~N} / \mathrm{mm}$ é atuada. A figura 6.1 ilustra o domínio fixo estendido e as condições de contorno aplicadas. Apesar de se esperar uma solução simétrica, nenhuma simetria foi imposta para estes resultados. 


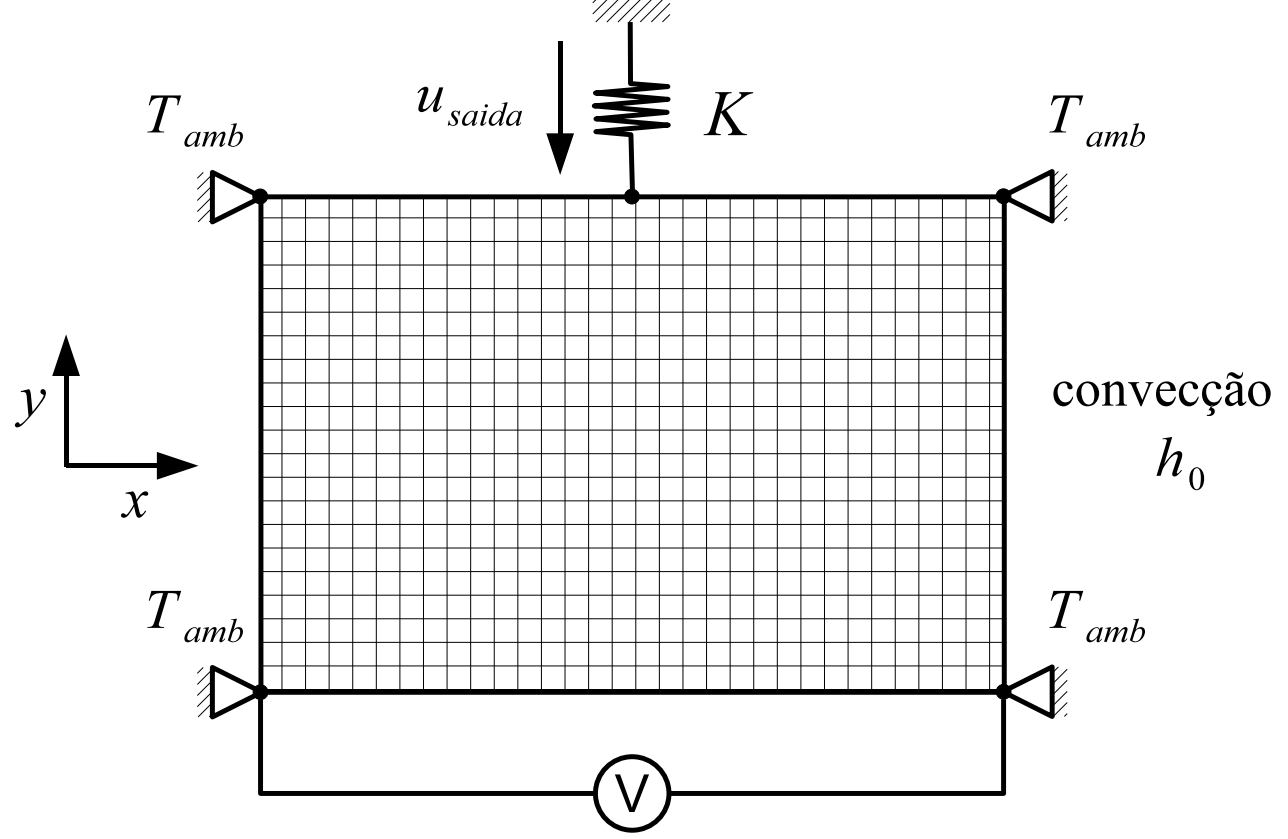

Figura 6.1: Domínio fixo estendido para o atuador de movimento inverso.

\subsubsection{Uso de dois materiais, além do vazio}

Como no problema resolvido por Yin e Ananthasuresh (2002), foram usados os mesmos dois materiais na otimização: silício e dióxido de silício. As propriedades de cada uma estão relacionadas na tabela 6.1.

Tabela 6.1: Propriedades dos Materiais - Silício e Dióxio de silício.

\begin{tabular}{|l|c|c|}
\hline Propriedades & Material 1 $(\mathrm{Si})$ & Material 2 $\left(\mathrm{SiO}_{2}\right)$ \\
\hline \hline Módulo de Elasticidade E $\left(\mathrm{N} / \mathrm{mm}^{2}\right)$ & $190 \times 10^{3}$ & $73 \times 10^{3}$ \\
Coeficiente de Poisson & 0,31 & 0,31 \\
Coeficiente de Expansão Térmica $\left(\mathrm{K}^{-1}\right)$ & $2,33 \times 10^{-6}$ & $0,55 \times 10^{-6}$ \\
Condutividade Elétrica $(1 / \Omega \cdot \mathrm{mm})$ & 0,1 & $1 \times 10^{-15}$ \\
Condutividade Térmica $(\mathrm{W} / \mathrm{K} \cdot \mathrm{mm})$ & 0,157 & $1,4 \times 10^{-3}$ \\
\hline
\end{tabular}

O modelo de interpolação de material utilizado foi definido de modo a criar picos onde as pseudo-densidades forem 1,0, representando o Material 1 (Silício), ou 2,0, representando o Material 2 (Dióxido de silício). Conforme já discutido sobre o processo de continuação (seção 4.2.2), inicia-se a otimização com um desvio padrão mais alto e termina-se com um desvio mais baixo de acordo com a equação (5.9). Todos os desvios padrão $\sigma_{m}$ e seus coeficientes de continuação $\omega_{D P_{m}}$ (com $m$ denotando o material referido) foram considerados iguais entre todos os materiais durante a otimização. A figura 6.2 ilustra a função de pico que interpola os módulos de Young dos dois materiais considerados (exceto o vazio) com diferentes desvios padrão.

Na primeira iteração do processo da otimização, todas as pseudo-densidades 


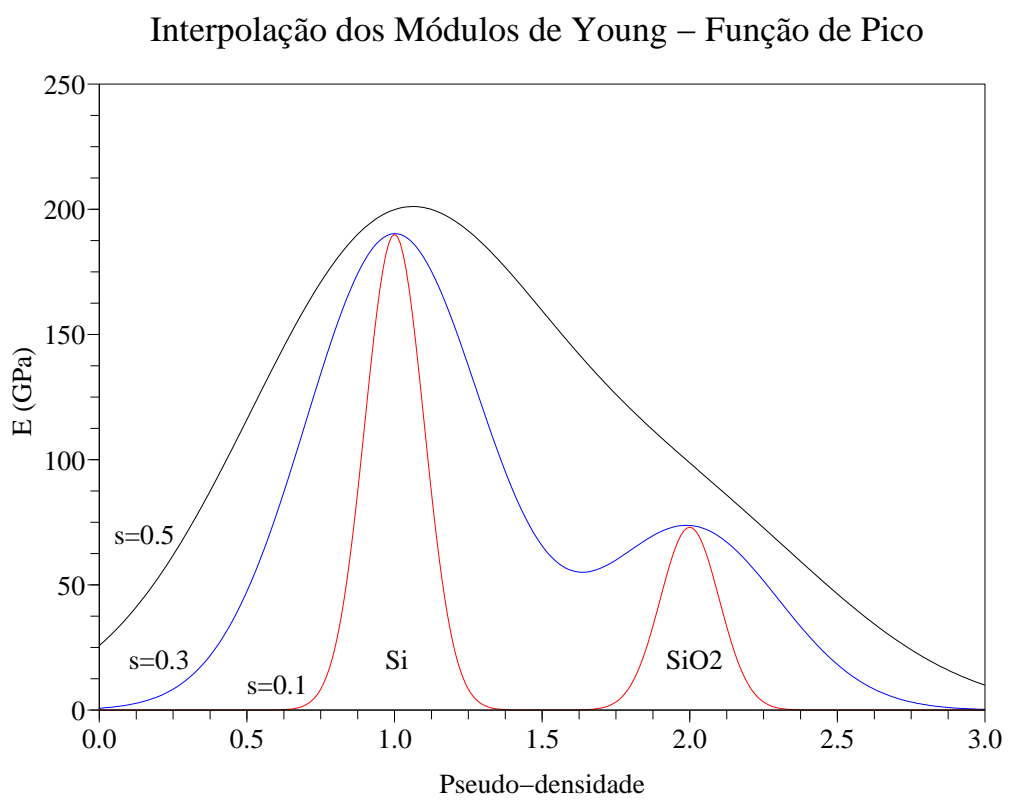

Figura 6.2: Interpolação dos Módulos de Young de $\mathrm{Si}$ e $\mathrm{SiO}_{2}-\sigma_{m}=0,5,0,3$ e $0,1(m=1$ e 2$)$.

foram consideradas uniformes e com valor igual a 1,5, valor escolhido por estar entre as posições dos dois picos $\left(\mu_{m}\right.$, para $m=1$ e 2$)$.

O filtro espacial foi utilizado com raio igual a 0,11 , de modo a abranger apenas os elementos imediatamente na sua vizinhança na discretização considerada.

\section{Sem Restrições de Volume dos Materiais}

Inicialmente, sem considerar as restrições de volume de cada material, uma topologia próxima do resultado de Yin e Ananthasuresh (2002) foi obtida após 200 iterações. Foram registrados os resultados até 220 iterações da rotina de otimização (que consumiram 11 minutos de processamento), desligando-se o filtro espacial a partir da iteração 150 do processo para que a quantidade de materiais intermediários fosse reduzida. É importante lembrar que o filtro atua de modo a reduzir os gradientes entre as variáveis de projeto próximas entre si. Os desvios padrão $\sigma_{m}$ variaram de 0,3 a 0,1 , utilizando-se um coeficiente de continuação $\omega_{D P_{m}}$ de 0,01 nesta redução, conforme a equação (5.9). Os desvios padrão $\sigma_{m}$ ficaram limitados a um valor mínimo de 0,1 , visto que os picos dos materiais do modelo de interpolação já se encontrariam bem pronunciados e separados. Um valor demasiadamente pequeno de $\sigma_{m}$ poderia comprometer possíveis refinamentos nas iterações finais de otimização, dado que os limites móveis são dependentes de $\sigma_{m}$ conforme a descrição da implementação do MOT (seção 5.2). A figura 6.3 mostra as topologias intermediárias encontradas antes da solução final e a figura 6.4, a 


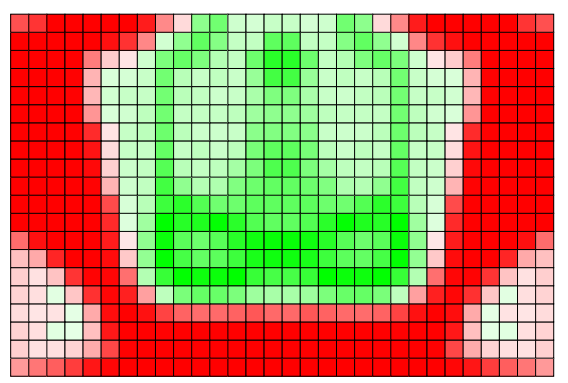

(a) 50 iterações

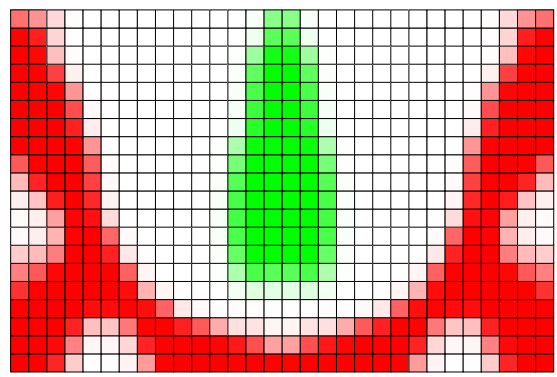

(c) 150 iterações

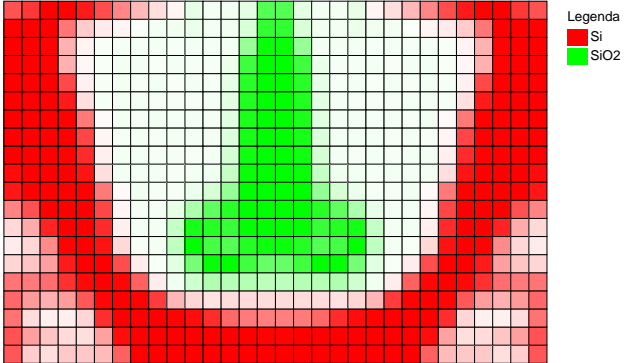

(b) 100 iterações

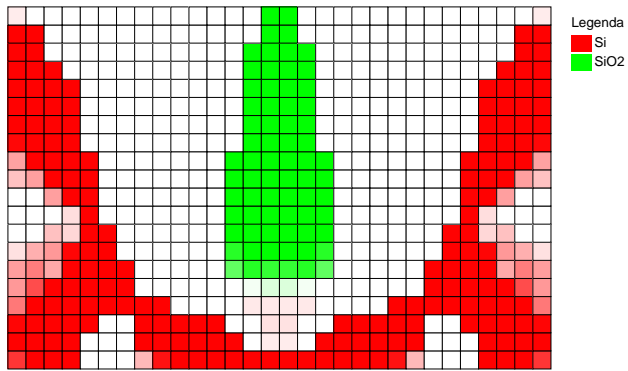

(d) 200 iterações

Figura 6.3: Atuador de movimento inverso - Topologias intermediárias (vermelho - $\mathrm{Si}$; verde - $\mathrm{SiO}_{2}$ ).

curva da função objetivo (o deslocamento de saída).

Sobre a topologia resultante (iteração 200), foi feita uma interpretação de modo a torná-la factível: os elementos com mais de $50 \%$ do material predominante tiveram a pseudo-densidade alterada para $100 \%$ desse mesmo material; caso contrário, recebe o valor do vazio para a pseudo-densidade. Além dessa interpretação, alguns elementos em locais estratégicos (como os pontos restritos em $x$ e $y$ e a ligação entre as estruturas em $\mathrm{Si}$ e em $\mathrm{SiO}_{2}$ ) foram fixos num determinado material, independente do valor da pseudo-densidade resultante da otimização. A topologia obtida e suas análises pelo MEF estão apresentadas na figura 6.5. As características desta topologia são mostradas na tabela 6.2.

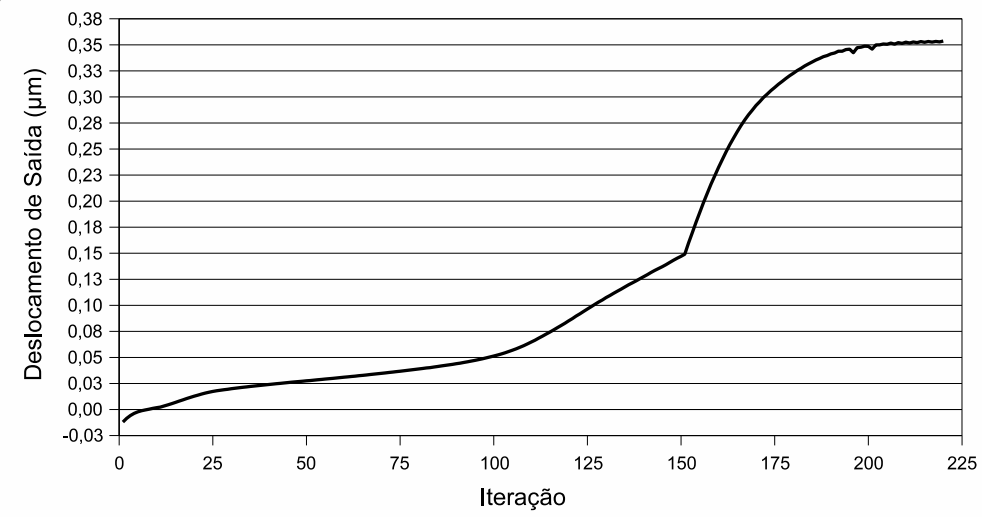

Figura 6.4: Atuador de movimento inverso - Função Objetivo. 


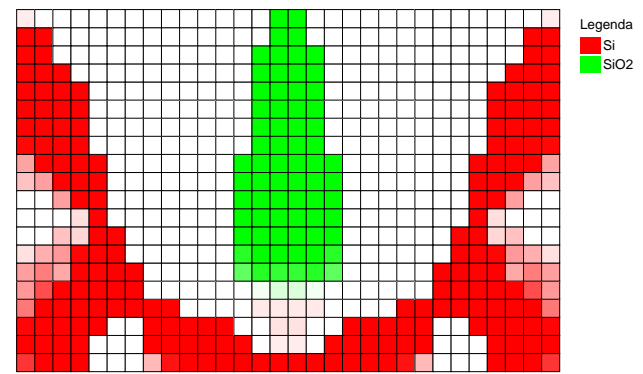

(a) Topologia resultante (vermelho - $\mathrm{Si}$; verde $-\mathrm{SiO}_{2}$ )

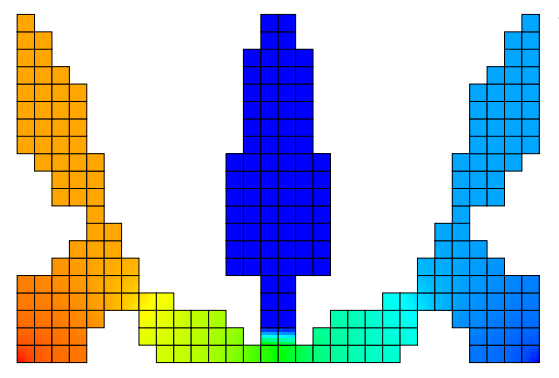

(c) Tensões Elétricas

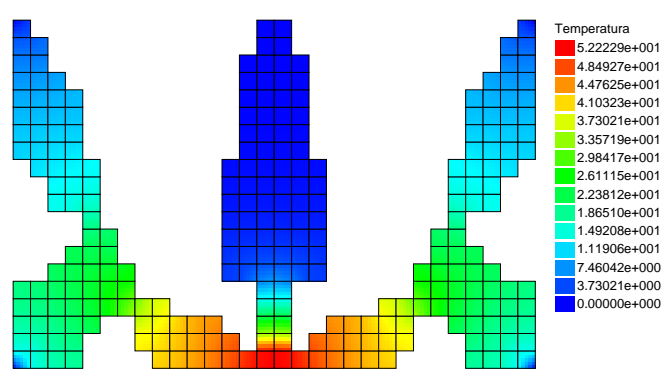

(e) Variações de Temperatura

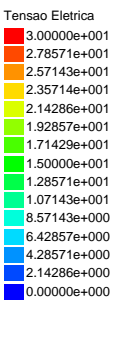

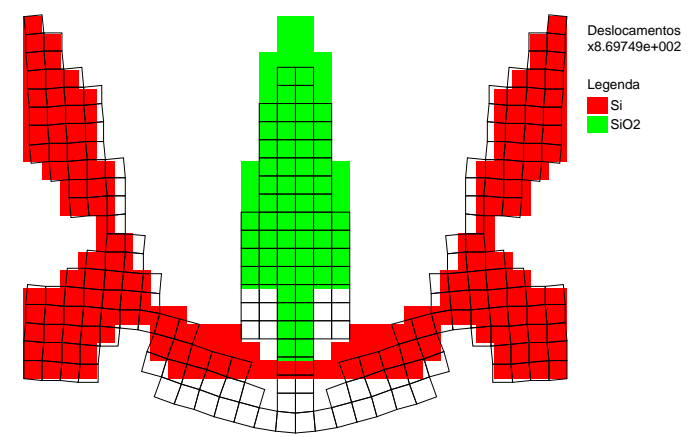

(b) Topologia interpretada e Deslocamentos

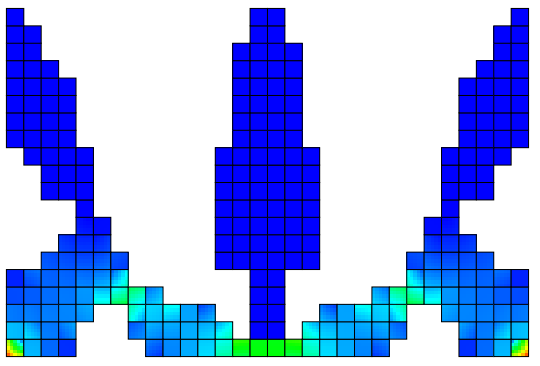

Densidade Correnti

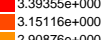
$2.906766+000$
$2.6636+000$
$2.42397 \mathrm{e}+000$ $2.18157 \mathrm{e}+000$ $1.93917 \mathrm{e}+000$
$-1.69678 \mathrm{e}+000$ $1.45438 \mathrm{e}+000$
$1.21198 \mathrm{e}+000$ $9.69587 \mathrm{e}-001$
$-7.27190 \mathrm{e}-001$

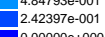

(d) Densidades de Corrente

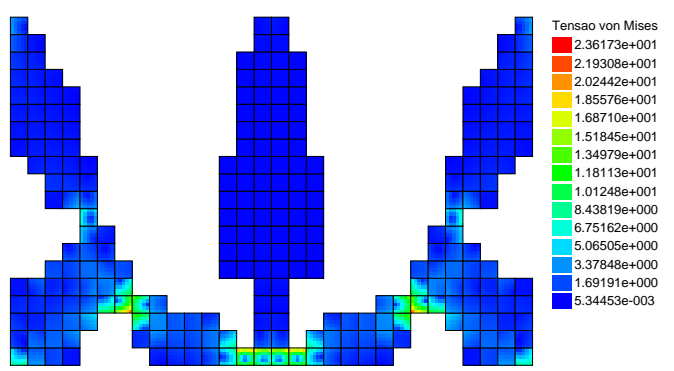

(f) Tensões de von Mises

Figura 6.5: Atuador de movimento inverso - Topologia final e Análises. 
Tabela 6.2: Atuador de movimento inverso - Características da topologia final.

\begin{tabular}{|l|c|}
\hline Característica & Valor \\
\hline \hline Deslocamento de Saída & $3,182 \times 10^{-1} \mu \mathrm{m}$ \\
Volumes Relativos & \\
- Silício & $30,67 \%$ \\
- Dióxido de silício & $13,00 \%$ \\
- Total & $\mathbf{4 3 , 6 7 \%}$ \\
\hline
\end{tabular}

Pode-se verificar que a topologia do mecanismo foi criada dispondo o silício, mais condutivo, de modo a realizar a atuação, aquecendo-se e expandindo. $\mathrm{O}$ dióxido de silício, um isolante elétrico e térmico, foi disposto sob a forma da haste que liga a parte em silício e o grau de liberdade de saída. Por ser isolante, permite que essa haste não se aqueça, o que poderia causar uma expansão no sentido contrário ao desejado e uma redução no deslocamento de saída.

\section{Com Restrições de Volume dos Materiais}

Para um controle melhor da topologia gerada, é interessante impor restrições de volume específicas para cada material. Assim, durante todo o processo de otimização, o volume de silício foi restrito a $30 \%$ do volume total do domínio fixo estendido e o volume do dióxido de silício, a 10\%, valores ligeiramente menores que os encontrados na topologia obtida sem restrições de volume dos materiais. Utilizando os mesmos parâmetros, a topologia ótima foi alcançada também após 200 iterações. A figura 6.6 mostra as topologias intermediárias encontradas antes da solução final e a figura 6.7 , a curva da função objetivo.

Como no caso anterior, a topologia resultante da iteração 200 foi interpretada da mesma maneira. A topologia obtida e suas análises pelo MEF estão apresentadas na figura 6.8. As características desta topologia são mostradas na tabela 6.3.

Tabela 6.3: Atuador de movimento inverso (com restrições de volume) Características da topologia final.

\begin{tabular}{|l|c|}
\hline Característica & Valor \\
\hline \hline Deslocamento de Saída & $4,067 \times 10^{-1} \mu \mathrm{m}$ \\
Volumes Relativos & \\
- Silício & $19,83 \%$ \\
- Dióxido de silício & $8,167 \%$ \\
- Total & $\mathbf{2 8 , 0 0 \%}$ \\
\hline
\end{tabular}

A solução encontrada utilizando restrições de volume por material possui um 


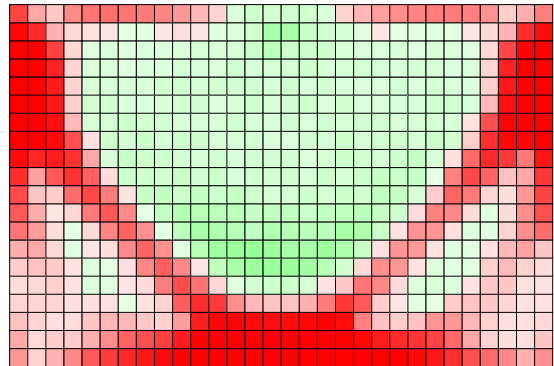

(a) 50 iterações

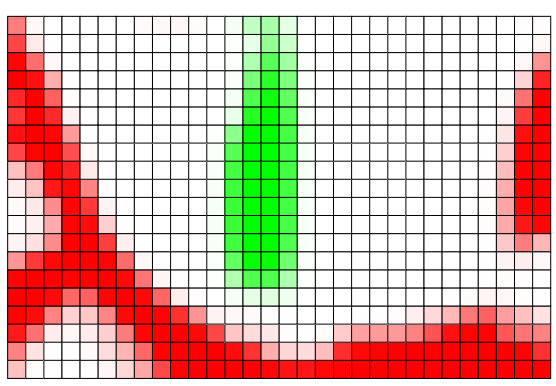

(c) 150 iterações

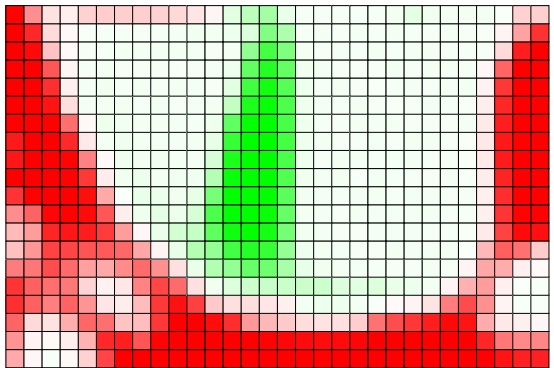

$\underset{\text { LiO2 }}{\text { Legenda }}$

(b) 100 iterações

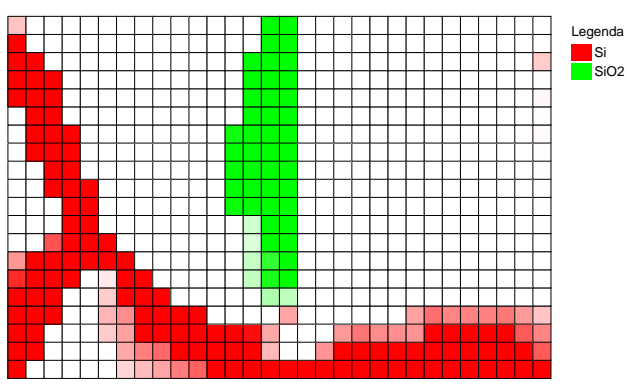

(d) 200 iterações

Figura 6.6: Atuador de movimento inverso (com restrições de volume) Topologias intermediárias (vermelho - Si; verde - $\mathrm{SiO}_{2}$ ).

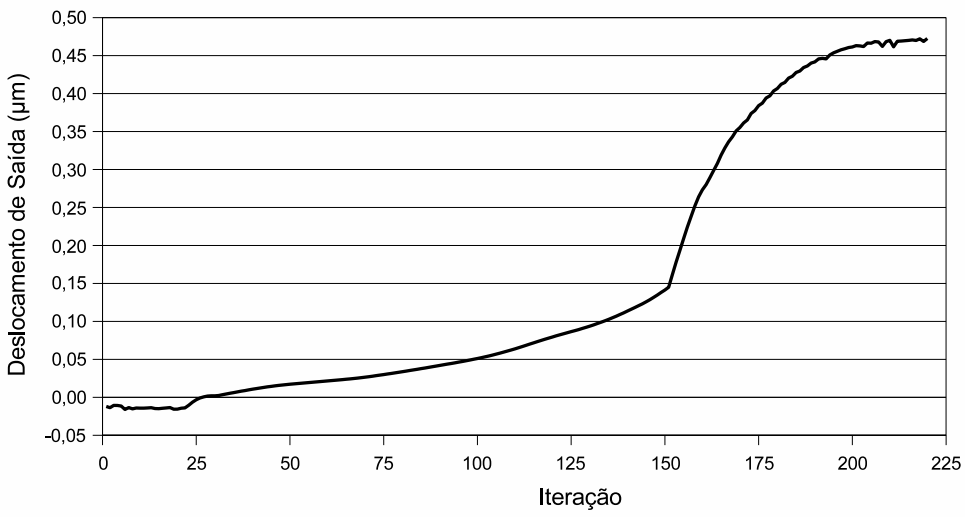

Figura 6.7: Atuador de movimento inverso (com restrições de volume) Função Objetivo. 


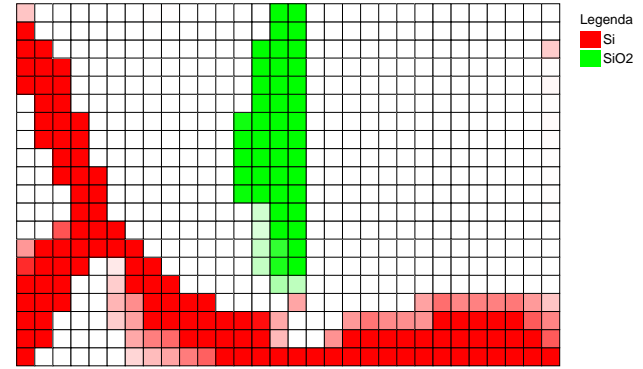

(a) Topologia resultante (vermelho - $\mathrm{Si}$; verde $-\mathrm{SiO}_{2}$ )

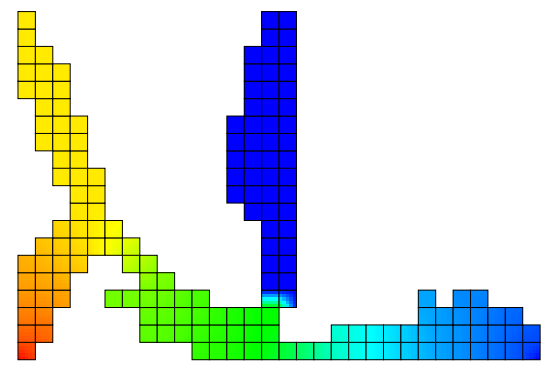

(c) Tensões Elétricas

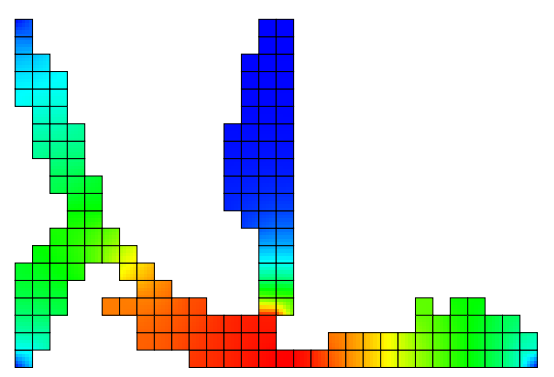
(e) Variações de Temperatura
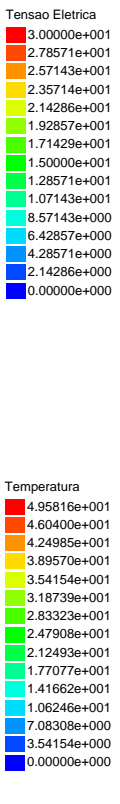

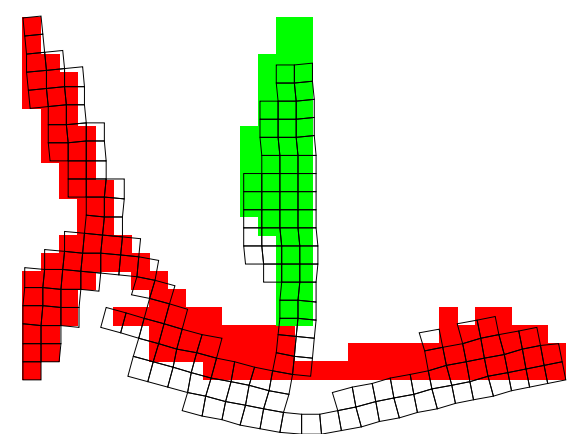

(b) Topologia interpretada e Deslocamentos

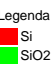

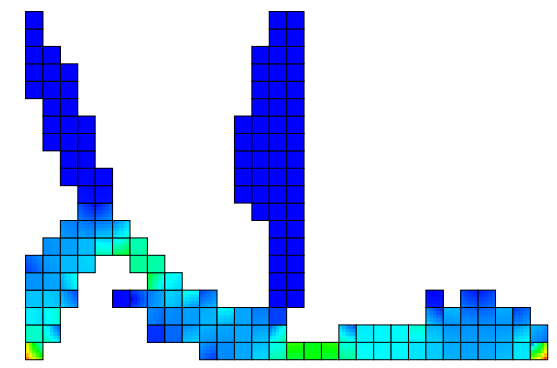

(d) Densidades de Corrente

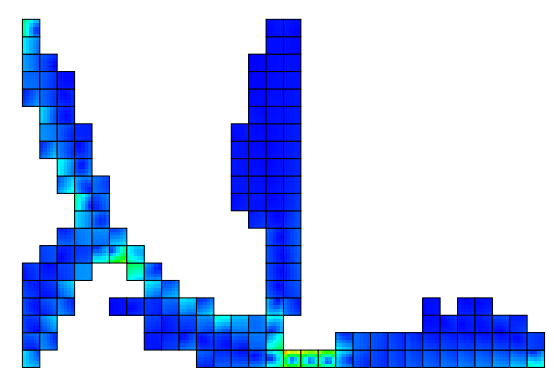

(f) Tensões de von Mises

Figura 6.8: Atuador de movimento inverso (com restrições de volume) Topologia final e Análises. 
deslocamento de saída mais de $25 \%$ maior, mas atuação sobre $u_{\text {saida }}$ pode ter movimento ortogonal acoplado, dependendo da rigidez $K$ da peça atuada, devido à assimetria do mecanismo. Apesar de não ser uma topologia simétrica igual à obtida por Yin e Ananthasuresh (2002), uma de suas metades é semelhante ao resultado da literatura e possui mesma funcionalidade. O aparecimento da assimetria devido à restrição de volume será comentado mais adiante, na seção 6.2 .

\subsubsection{Uso de três materiais, além do vazio}

O mesmo tipo de atuador também foi projetado com a consideração de um material adicional, o níquel, cujas propriedades são apresentadas na tabela 6.4.

Tabela 6.4: Propriedades dos Materiais - Níquel.

\begin{tabular}{|l|c|}
\hline Propriedades & Material 3 (Níquel) \\
\hline \hline Módulo de Elasticidade E $\left(\mathrm{N} / \mathrm{mm}^{2}\right)$ & $188 \times 10^{3}$ \\
Coeficiente de Poisson & 0,31 \\
Coeficiente de Expansão Térmica $\left(\mathrm{K}^{-1}\right)$ & $15 \times 10^{-6}$ \\
Condutividade Elétrica $(1 / \Omega \cdot \mathrm{mm})$ & $6,37 \times 10^{3}$ \\
Condutividade Térmica $(\mathrm{W} / \mathrm{K} \cdot \mathrm{mm})$ & $90,7 \times 10^{-3}$ \\
\hline
\end{tabular}

O mesmo domínio fixo estendido e condições de contorno foram usados, com exceção do valor da tensão elétrica $V$ aplicada, que foi de $0,1 \mathrm{~V}$.

Em relação ao modelo de interpolação de material utilizado, foi acrescentado um termo com pico para a pseudo-densidade 3,0, representando o Material 3 (Níquel). Os desvios padrão $\sigma_{m}$ se iniciaram em 0,5 , caindo para 0,1 gradualmente durante a otimização. A figura 6.9 ilustra a função de pico que interpola os módulos de Young dos três materiais considerados, além do vazio, com diferentes desvios padrão.

Na primeira iteração do processo da otimização, todas as pseudo-densidades foram consideradas uniformes e com valor igual a 2,0, valor escolhido por ser a média das posições dos três picos $\left(\mu_{m}\right.$, para $m=1,2$ e 3$)$. As restrições de volume dos materiais não foram utilizadas.

A topologia otimizada foi encontrada após 360 iterações, desativando-se o filtro espacial desde a iteração 200 do processo. A figura 6.10 mostra as topologias intermediárias encontradas antes da solução final e a figura 6.11, a curva da função objetivo.

A topologia da iteração 360 apresenta várias juntas onde o contato entre um 


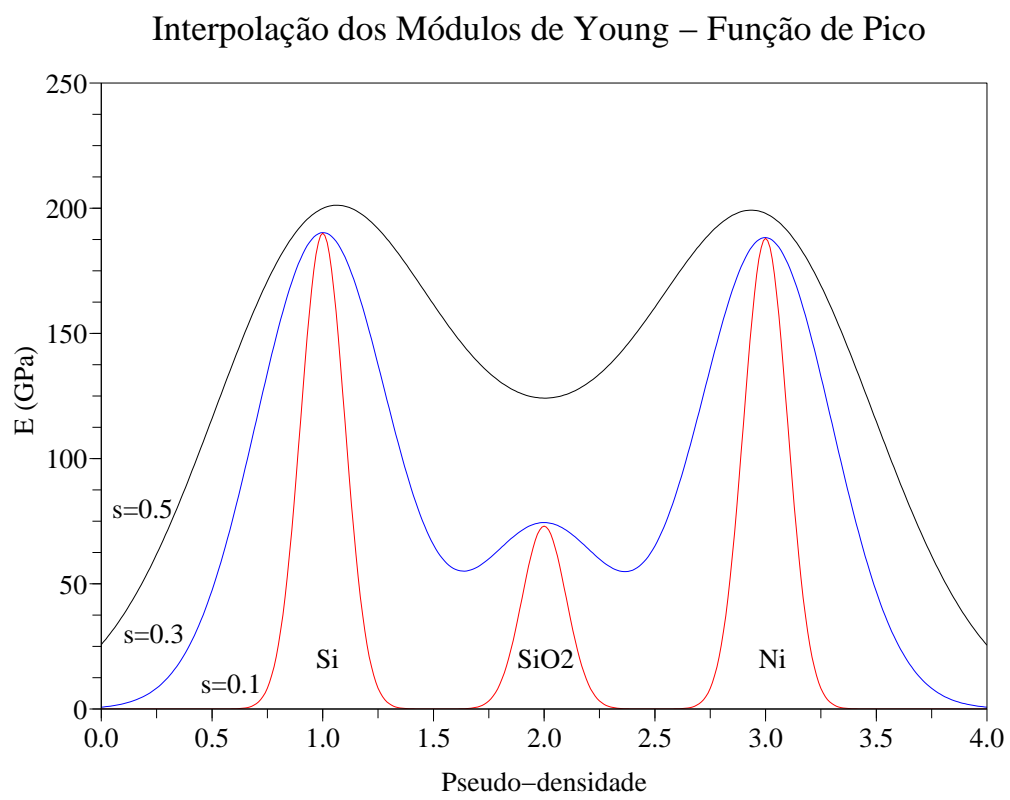

Figura 6.9: Interpolação dos Módulos de Young de $\mathrm{Si}, \mathrm{SiO}_{2}$ e $\mathrm{Ni}-\sigma_{m}=0,5$, 0,3 e $0,1(m=1,2$ e 3$)$.

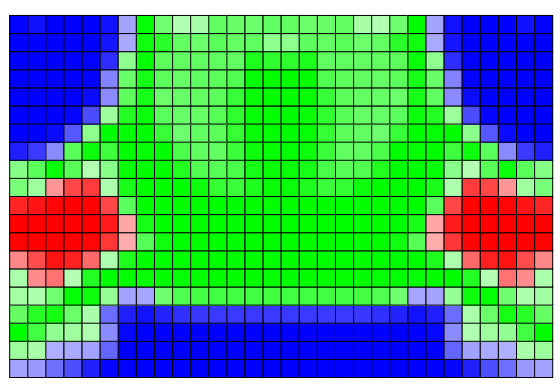

(a) 90 iterações

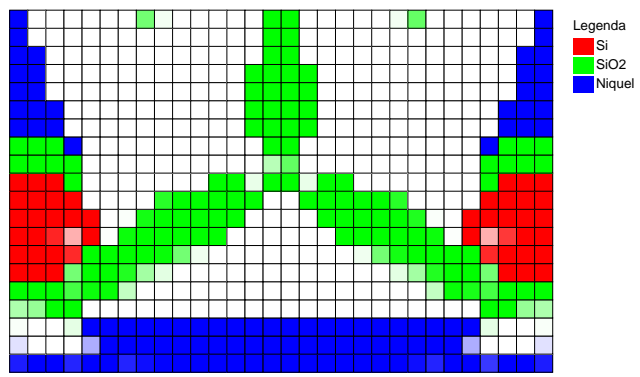

(c) 270 iterações

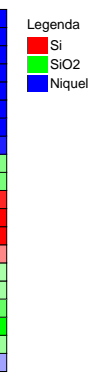

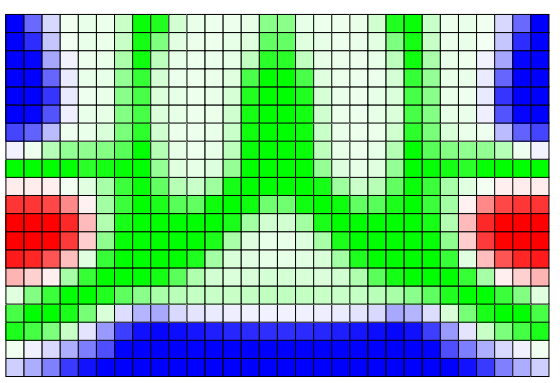

(b) 180 iterações

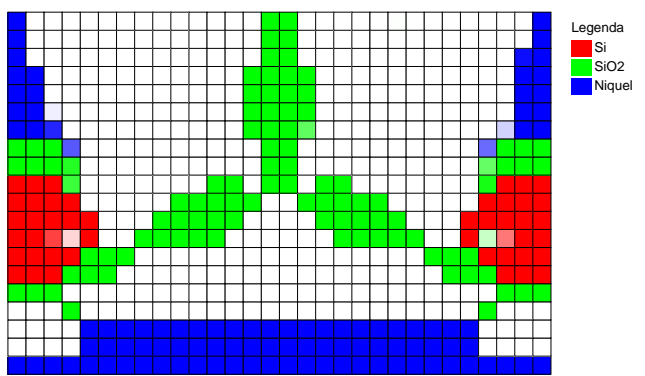

(d) 360 iterações

Figura 6.10: Atuador de movimento inverso (3 materiais) - Topologias intermediárias (vermelho - $\mathrm{Si}$; verde - $\mathrm{SiO}_{2}$; azul - Ni). 


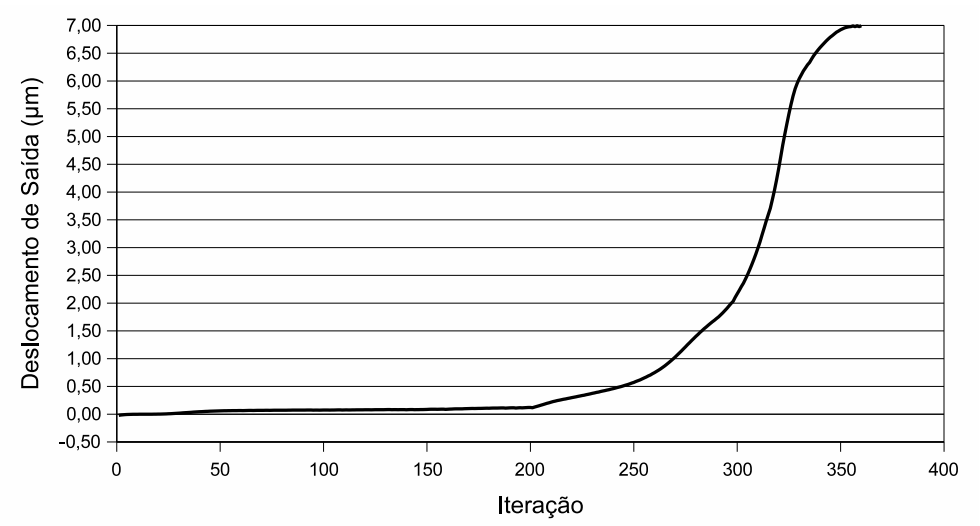

Figura 6.11: Atuador de movimento inverso (3 materiais) - Função Objetivo.

elemento e outro se dá apenas por um nó, um problema comum em otimizações cujo objetivo é maximizar um funcional relacionado a uma força ou deslocamento de saída. Uma solução para esse problema é dada por Cardoso e Fonseca (2004) e consiste em projetar estruturas flexíveis com um máximo de absorção da energia de deformação, o que não será abordado neste trabalho. Então, para se obter uma topologia factível, o resultado foi interpretado de modo a se eliminar individualmente cada uma dessas ocorrências. A perda da flexibilidade proporcionada por esse tipo de junta acarretou numa queda significativa no deslocamento de saída, reduzido a menos de um terço do resultado obtido pela rotina. A topologia resultante da otimização e a interpretada, juntamente com as análises pelo MEF para o caso interpretado, estão apresentadas na figura 6.12. As características desta topologia são mostradas na tabela 6.5.

Tabela 6.5: Atuador de movimento inverso (3 materiais) - Características da topologia final.

\begin{tabular}{|l|c|}
\hline Característica & Valor \\
\hline \hline Deslocamento de Saída & $2,149 \mu \mathrm{m}$ \\
& \\
Volumes Relativos & \\
- Silício & $7,83 \%$ \\
- Dióxido de silício & $17,83 \%$ \\
- Níquel & $16,67 \%$ \\
- Total & $\mathbf{4 2 , 3 3 \%}$ \\
\hline
\end{tabular}

A introdução do níquel alterou a funcionalidade do mecanismo. Devido à maior condutividade elétrica e ao maior coeficiente de expansão térmica, foi empregado entre os nós de aplicação da tensão elétrica, substituindo o silício como principal material condutivo e responsável pela atuação eletrotermomecânica. O silício, com módulo de Young similar ao níquel (e mais elevado que o do dióxido de silício) e menores condutividades elétrica e térmica, teve participação mais 


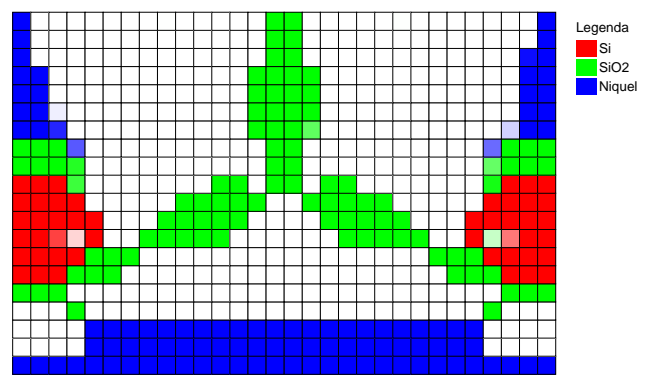

(a) Topologia resultante (vermelho - $\mathrm{Si}$; verde $-\mathrm{SiO}_{2} ;$ azul - Ni)

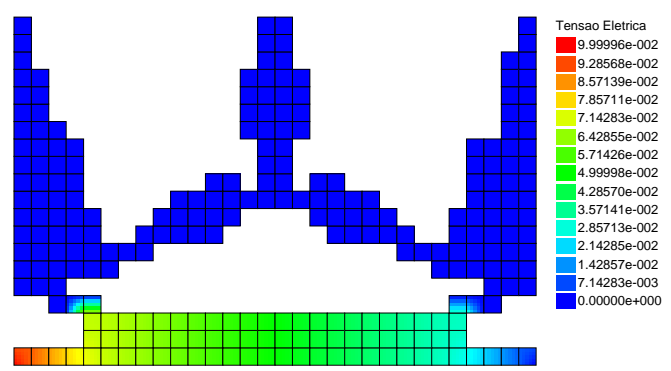

(c) Tensões Elétricas

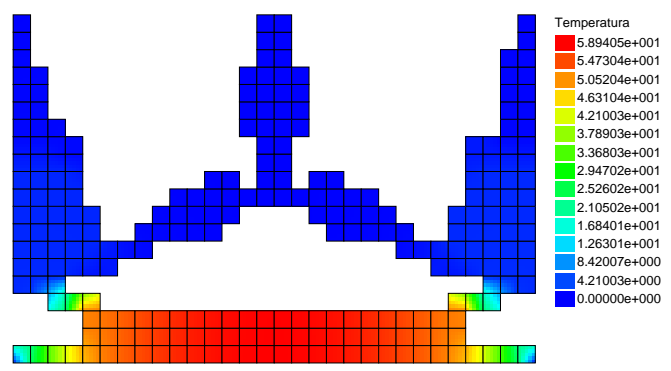

(e) Variações de Temperatura

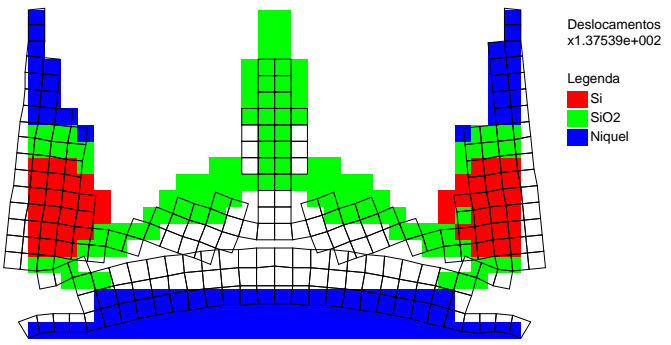

(b) Topologia interpretada e Deslocamentos

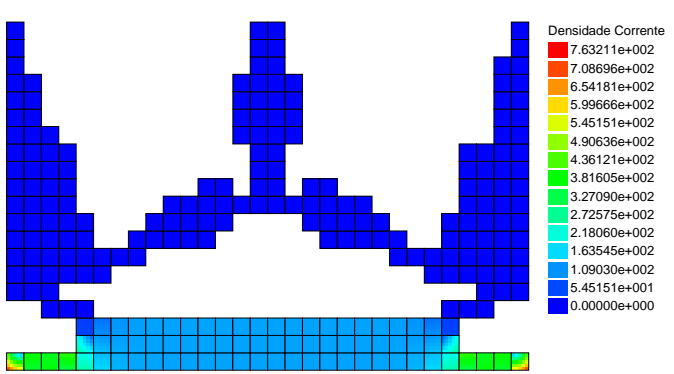

(d) Densidades de Corrente

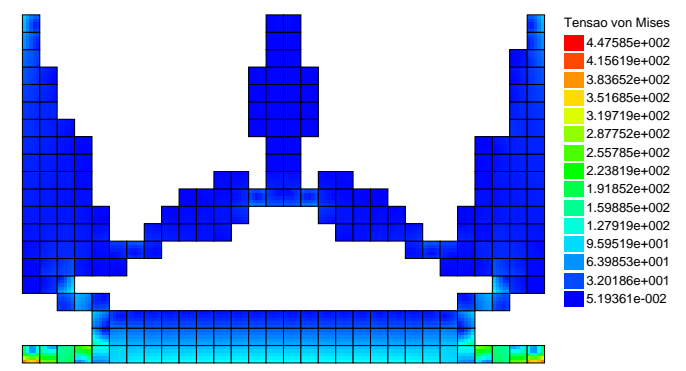

(f) Tensões de von Mises

Figura 6.12: Atuador de movimento inverso (3 materiais) - Topologia final e Análises. 


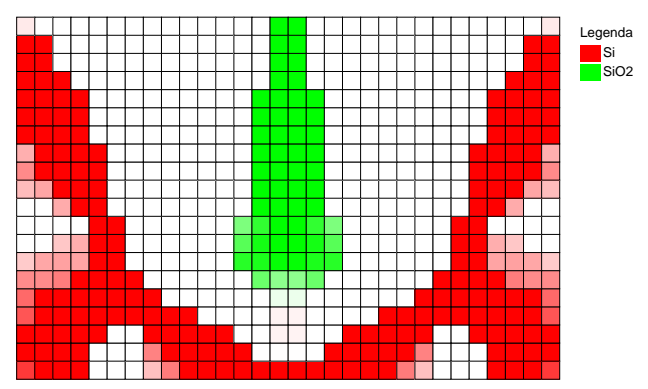

(a) $K=0,2 \mathrm{~N} / \mathrm{mm}$

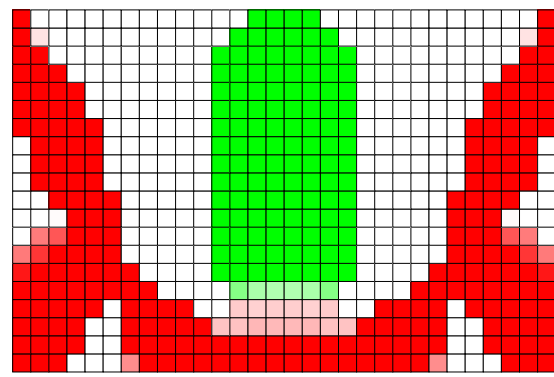

(c) $K=200 \mathrm{~N} / \mathrm{mm}$

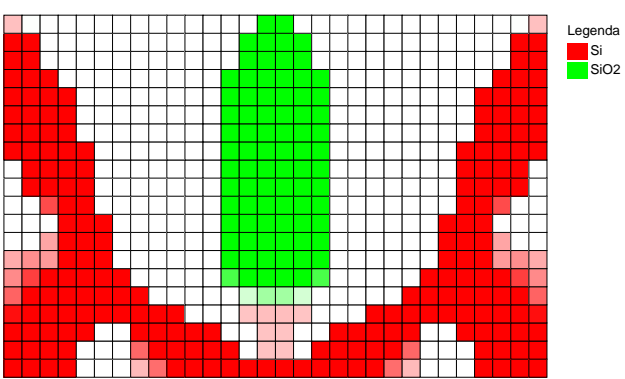

(b) $K=20 \mathrm{~N} / \mathrm{mm}$

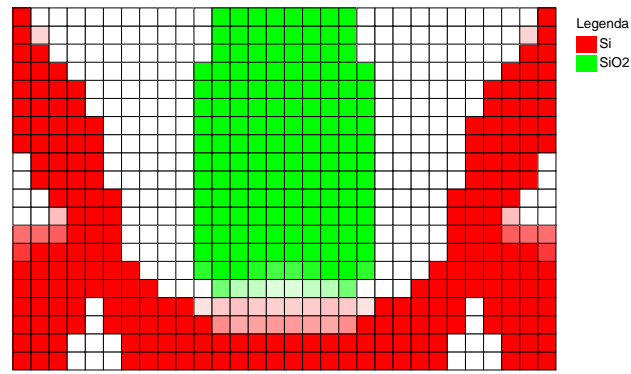

(d) $K=2.000 \mathrm{~N} / \mathrm{mm}$

Figura 6.13: Volume Relativo dos resultados para o atuador de movimento inverso.

estrutural no projeto.

\subsection{Influência dos Parâmetros da Otimização}

Os parâmetros da otimização que tiveram a sua influência investigada foram: rigidez da peça atuada, restrições de volume, uso do filtro espacial, coeficiente de continuação e parâmetros da função de pico (média e desvios padrão).

\subsubsection{Rigidez da Peça Atuada}

A partir dos parâmetros usados no projeto do atuador de movimento inverso sem restrição de material (seção 6.1), foram realizadas outras otimizações modificando-se o valor da rigidez $K$ da peça atuada pelo deslocamento de saída em diversas grandezas. Originalmente, $K$ foi considerado igual a $2 \mathrm{~N} / \mathrm{mm}$. As topologias resultantes, impondo simetria entre as metades esquerda e direita, estão apresentadas na figura 6.13.

Conforme discutido na seção 3.4.3, o menor valor de $K$ levou a uma estrutura de menor integridade, mecanismo onde as duas partes estão ligadas por elementos com densidades muito baixas de material. Aumentando-se a rigidez $K$, são obtidos mecanismos mais robustos, com uma rigidez estrutural maior por se 
privilegiar a força exercida pelo mecanismo em relação ao deslocamento de saída. A tabela 6.6 mostra os deslocamentos de saída das topologias finais relacionados a cada valor de $K$ empregado.

Tabela 6.6: Atuador de movimento inverso - Rigidez da peça atuada e deslocamentos de saída da topologia final.

\begin{tabular}{|c|c|}
\hline Rigidez $(\mathrm{N} / \mathrm{mm})$ & Deslocamento $(\mu \mathrm{m})$ \\
\hline \hline 0,2 & $3,488 \times 10^{-1}$ \\
20 & $2,244 \times 10^{-1}$ \\
200 & $7,048 \times 10^{-2}$ \\
2000 & $9,906 \times 10^{-3}$ \\
\hline
\end{tabular}

\subsubsection{Restrições de Volume por Material}

Os dois resultados apresentados de atuadores de movimento inverso (seção 6.1) permitem verificar que a inclusão das restrições de volume por material (apresentada na seção 5.1.1) pode alterar o resultado final, mesmo que estas restrições não estejam ativas ao término da otimização. Nesse caso, as restrições de volume não permitiram uma topologia simétrica. A figura 6.14 apresenta os volumes relativos de cada material e total durante a otimização.

Observa-se que as restrições de volume encontram-se ativas (com o volume do Si em $30 \%$ do volume total e do $\mathrm{SiO}_{2}$ em 10\%) durante o processo de otimização, o que leva a concluir que as restrições de volume alteraram o máximo local para onde a otimização caminhava em relação ao caso onde as restrições de volume não foram utilizadas. Uma comparação entre as topologias intermediárias dos dois casos (figuras 6.3 e 6.6) permite chegar à mesma conclusão. Para se obter uma topologia simétrica, pode-se fazer uma continuação da restrição de volume máximo, começando a otimização com restrições de volume altas e reduzindo-as gradualmente com o decorrer das iterações até a obtenção de uma topologia que não violasse as restrições de volume desejadas ao final do processo. A atualização dos volumes máximos de cada material em cada iteração pode ser vista na seção 5.2 (equação (5.10)). Com volumes máximos $\Omega_{s_{m}}$ iniciando com valor correspondente a $100 \%$ do volume total e coeficientes de continuação $\omega_{R V_{m}}$ iguais a 0,02 (ambos para cada material $m$ ), o resultado mostra uma recuperação da simetria (que não foi imposta nesse caso) e do máximo local, como pode ser visto na figura 6.15 juntamente com o resultado obtido sem restrições de volume. Os volumes relativos dos materiais são mostrados na figura 6.16. 


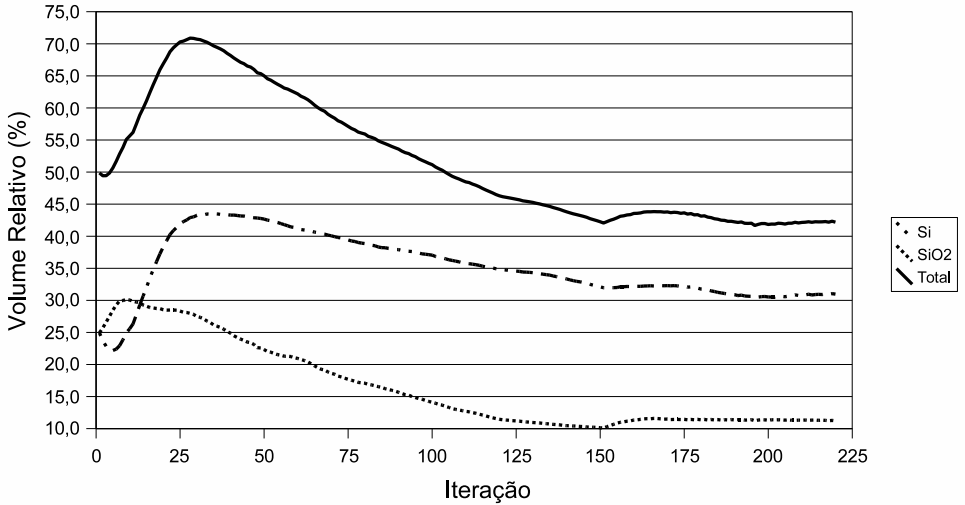

(a) Sem Restrições de Volume

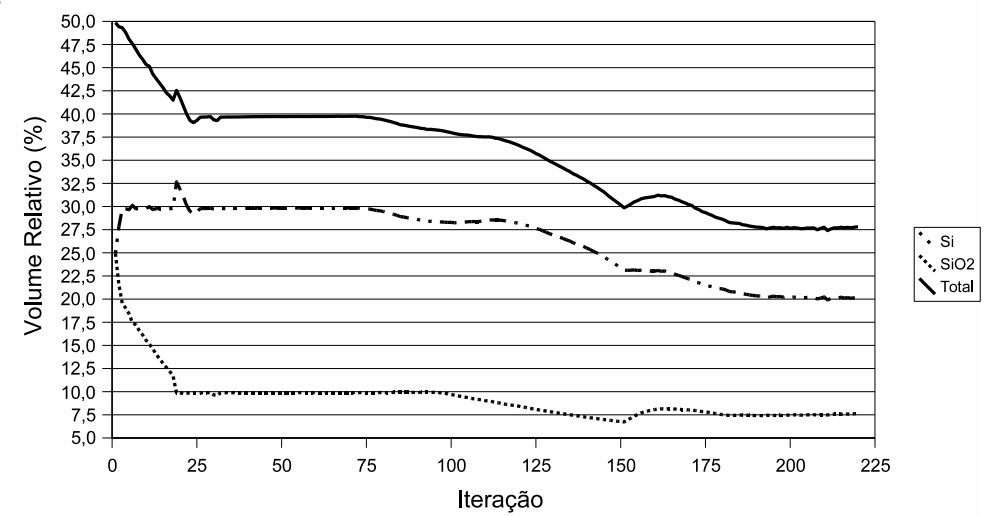

(b) Com Restrições de Volume

Figura 6.14: Volumes Relativos dos resultados para o atuador de movimento inverso.

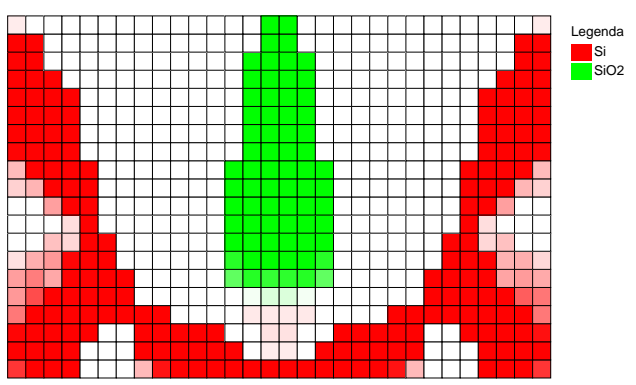

(a) Com restrições de volume continuadas

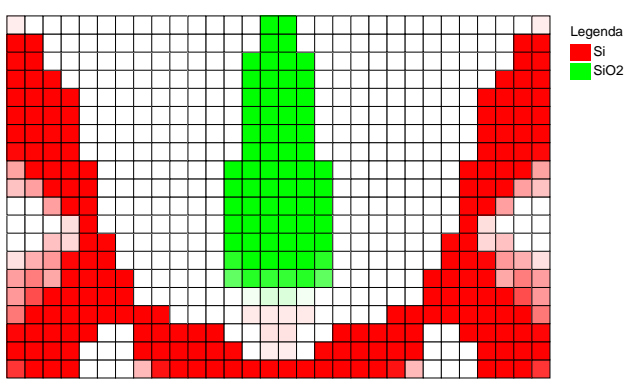

(b) Sem restrições de volume

Figura 6.15: Atuador de movimento inverso - Comparação de topologias (restrições de volume variadas continuamente). 


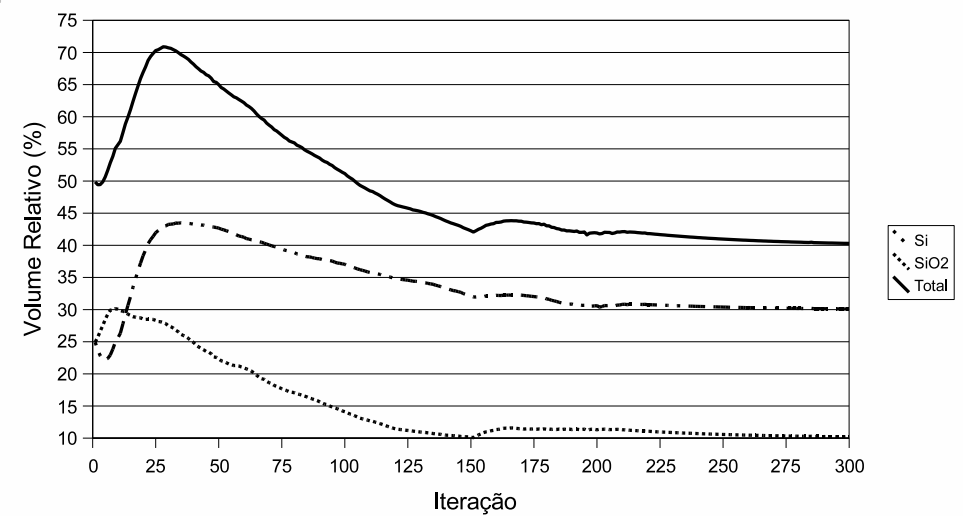

Figura 6.16: Volumes Relativos para o atuador de movimento inverso (restrições de volume variadas continuamente).

\subsubsection{Uso do Filtro Espacial}

Na seção 3.4.1, o uso de um filtro espacial é citado como possível solução para atenuar o problema da dependência de malha. Para verificar essa conseqüência do uso do filtro, o problema de otimização do atuador de movimento inverso em silício e dióxido de silício, sem restrições de volume dos materiais, da seção 6.1 foi resolvido para uma discretização quatro vezes maior, com 2400 elementos. As topologias intermediárias estão na figura 6.17 e uma comparação das topologias finais com as duas diferentes discretizações pode ser vista na figura 6.18.

Pela figura 6.18, verifica-se que o refinamento da discretização do domínio fixo estendido refinou a solução, gerando essencialmente a mesma topologia em ambos os casos.

Como descrito na seção 5.3, o filtro espacial altera os limites móveis originais de um elemento $e$ de modo a limitar os gradientes de material entre ele e os elementos próximos, dentro do raio de ação do filtro. Por causa do seu princípio de funcionamento, o filtro poderia retardar a convergência da topologia. Isso fica evidente nas curvas das funções objetivo (figura 6.4 e 6.7), onde pode-se ver uma descontinuidade na derivada das duas curvas da função objetivo na iteração 150, a partir da qual o filtro foi desativado. Para verificar esse efeito, a otimização do atuador de movimento inverso, sem restrição de volume, foi refeita com uma diferença: o filtro foi desativado na iteração 100. Os resultados estão apresentados nas figuras 6.19 e 6.20 .

A topologia ótima foi obtida a partir da iteração 240 (ainda com estruturas espúrias que são removidas até a iteração 300), 40 iterações mais tarde que se o filtro fosse desativado na iteração 150. O filtro pode ter um efeito retardador na convergência a princípio, mas existe a possibilidade de estar guiando a 


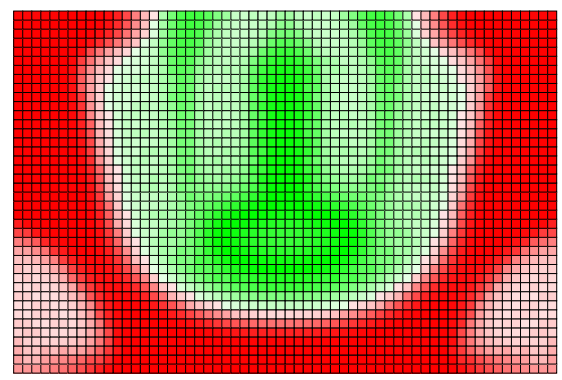

(a) 50 iterações

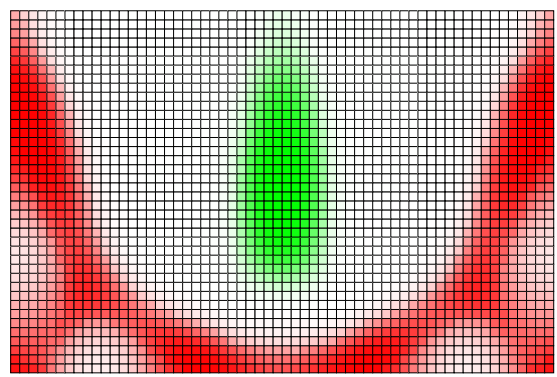

(c) 150 iterações

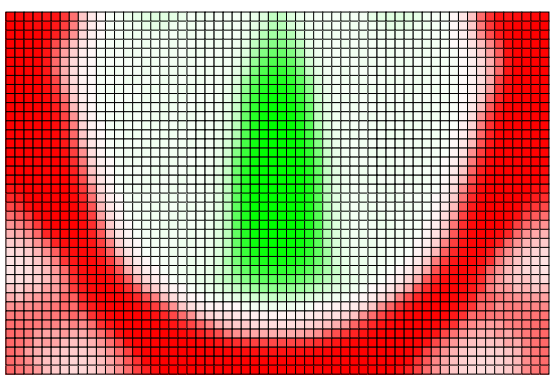

$\underset{\text { Lio2 }}{\text { Legenda }}$

$\underset{\text { Legenda }}{\text { Si }}$

(b) 100 iterações

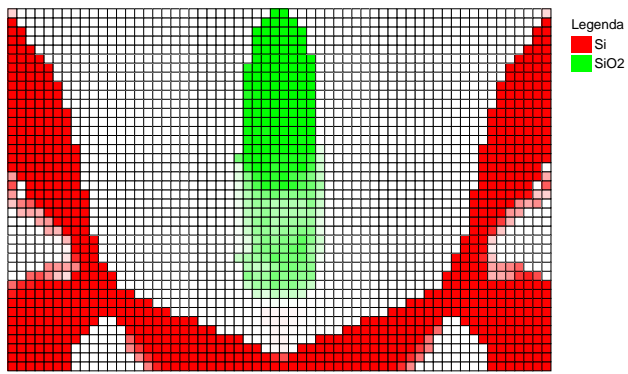

(d) 200 iterações

Figura 6.17: Atuador de movimento inverso (com maior discretização) Topologias intermediárias (vermelho - $\mathrm{Si}$; verde - $\mathrm{SiO}_{2}$ ).

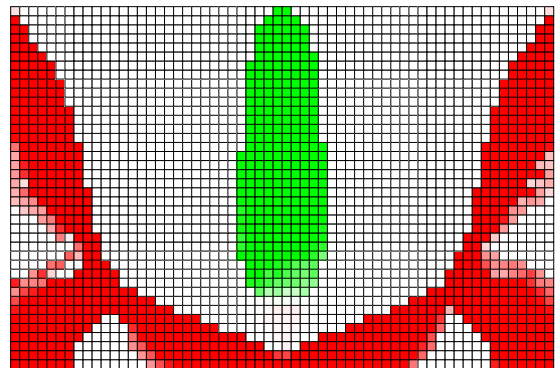

(a) 2400 elementos

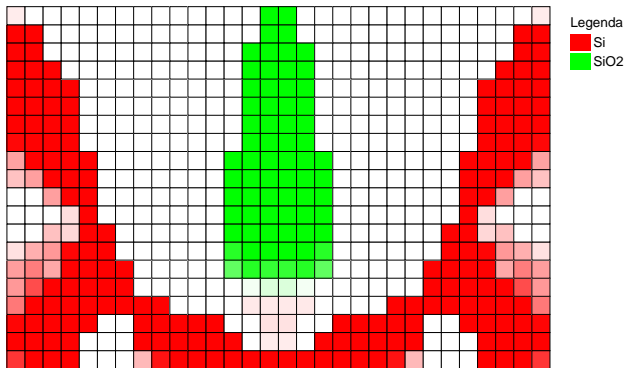

(b) 600 elementos

Figura 6.18: Atuador de movimento inverso (com maior discretização) Comparação de topologias (diferentes discretizações). 


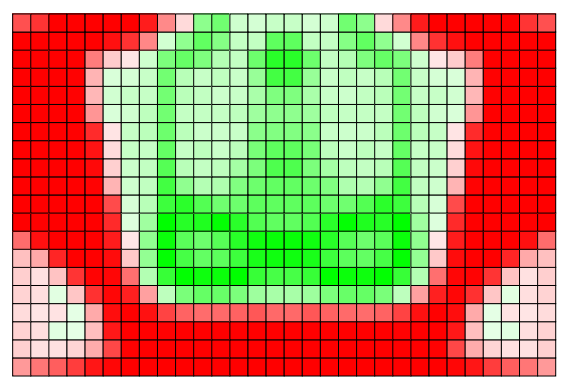

(a) 50 iterações

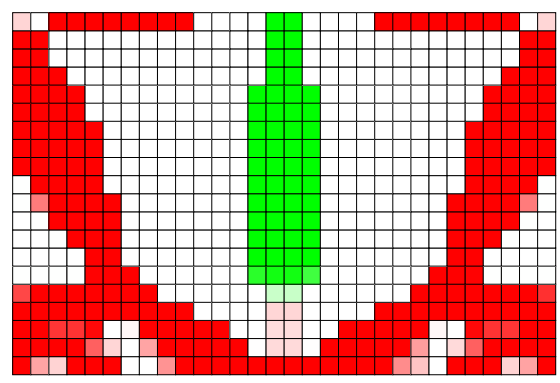

(c) 170 iterações $\stackrel{\substack{\text { Legenda } \\ \text { Si } \\ \text { Sio2 }}}{\text { Sions }}$

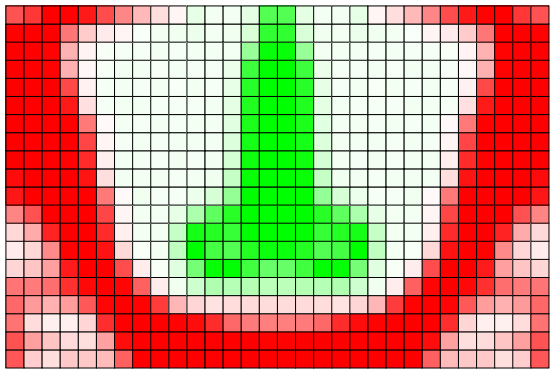

$\underset{\substack{\text { Legenda } \\ \text { Sio2 }}}{\text { sion }}$

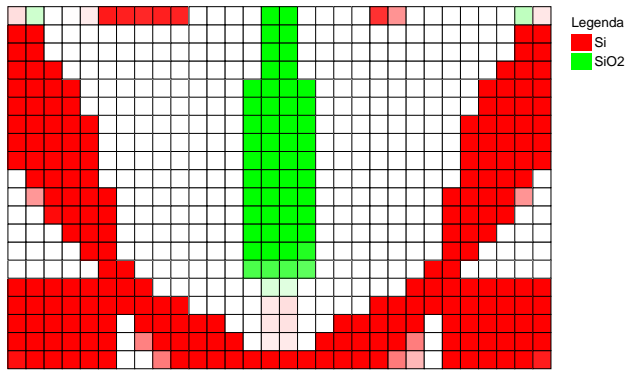

(d) 240 iterações

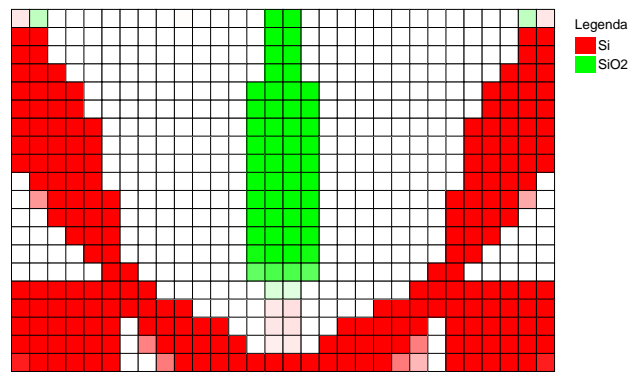

(e) 300 iterações

Figura 6.19: Atuador de movimento inverso (com filtro até iteração 100) Topologias intermediárias (vermelho - $\mathrm{Si}$; verde - $\mathrm{SiO}_{2}$ ).

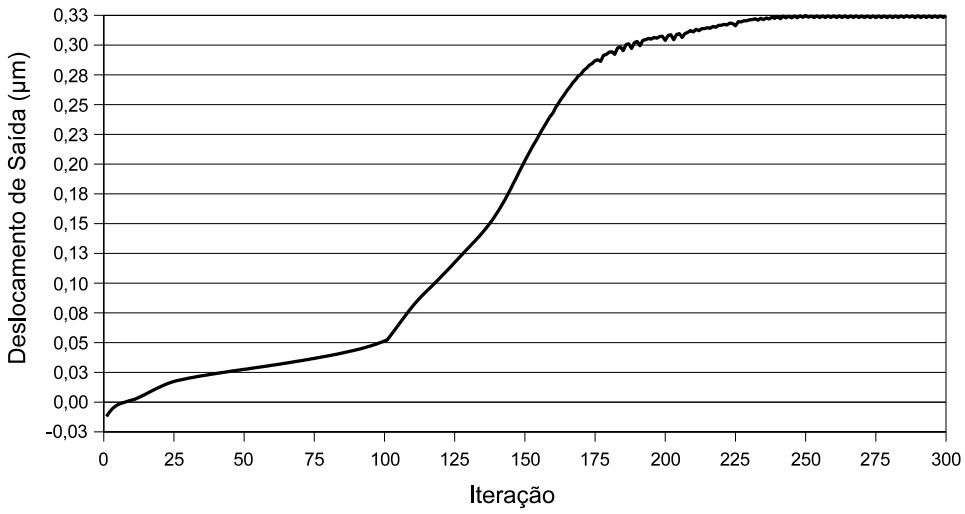

Figura 6.20: Atuador de movimento inverso (com filtro até iteração 100) Função Objetivo. 


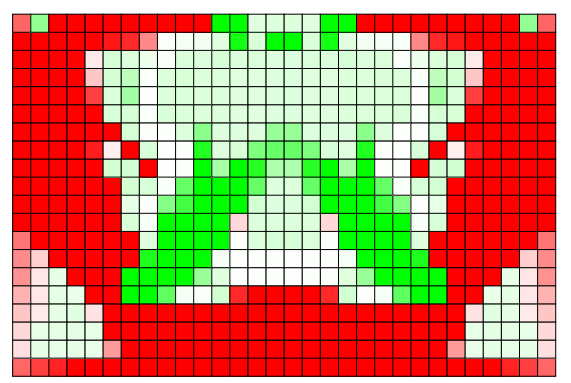

(a) 60 iterações

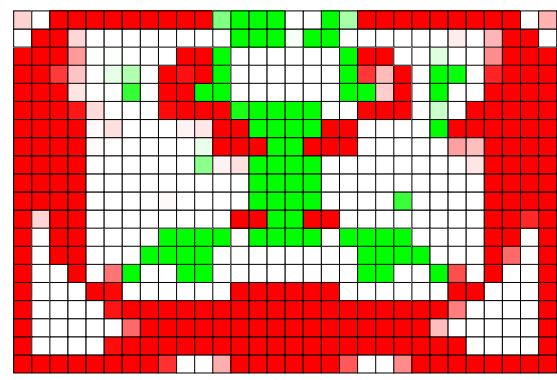

(c) 180 iterações

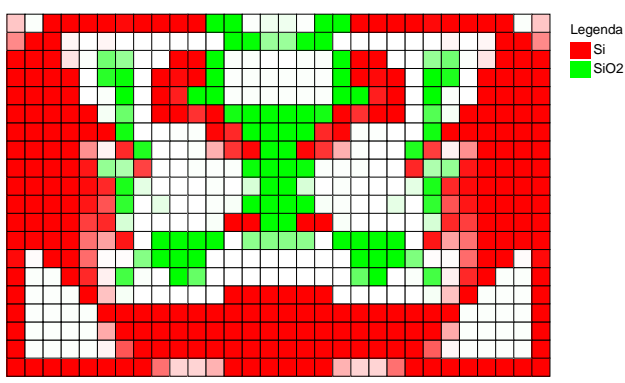

(b) 120 iterações

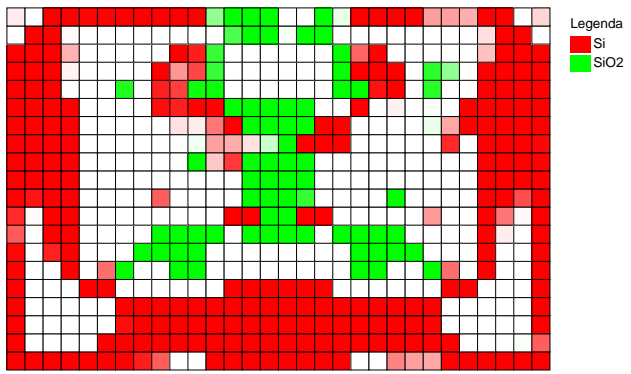

(d) 240 iterações

Figura 6.21: Atuador de movimento inverso (sem filtro) - Topologias intermediárias (vermelho - $\mathrm{Si}$; verde - $\mathrm{SiO}_{2}$ ).

otimização mais rapidamente para a topologia ótima. Se desativado muito antes da otimização estar mais bem definida em direção a um determinado máximo local, pode ser necessário mais iterações para se chegar a uma convergência.

Executando novamente a rotina de otimização com e sem restrições de volume por material, mas sem usar o filtro espacial em qualquer iteração, obtém-se os resultados mostrados da figura 6.21 a 6.24 .

Ambas as topologias resultantes dos processos sem o uso de filtro são bem complexas e tem um número considerável de estruturas desconexas ou espúrias. Pode-se perceber que, além de controlar dependência de malha (porque seu raio de

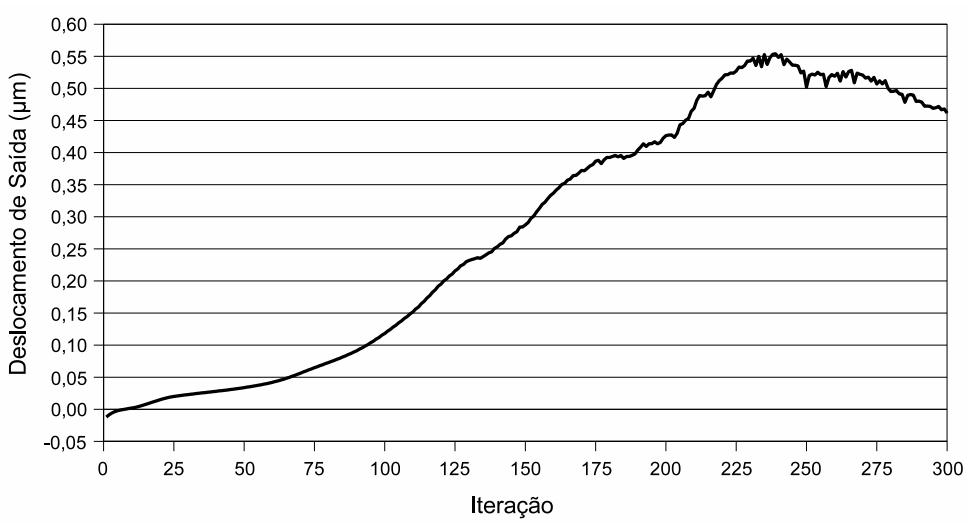

Figura 6.22: Atuador de movimento inverso (sem filtro) - Função Objetivo. 


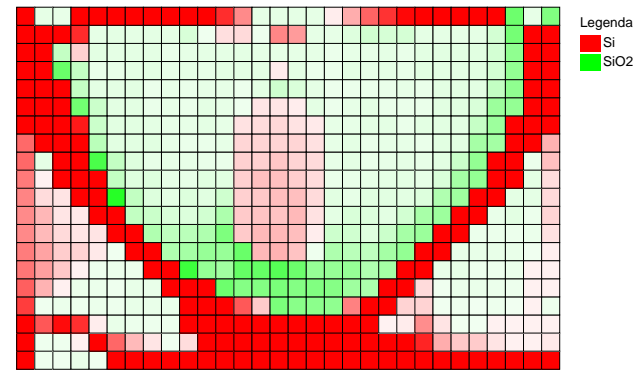

(a) 75 iterações

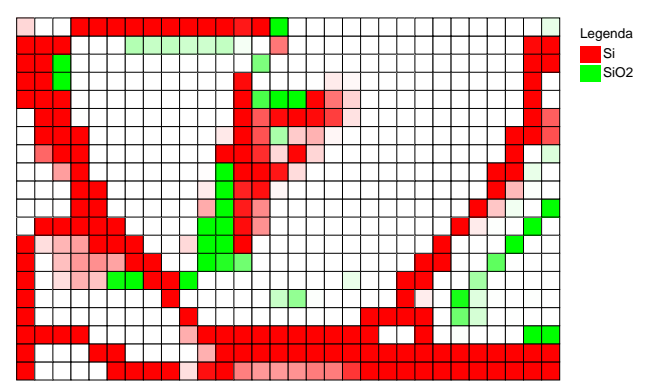

(c) 225 iterações

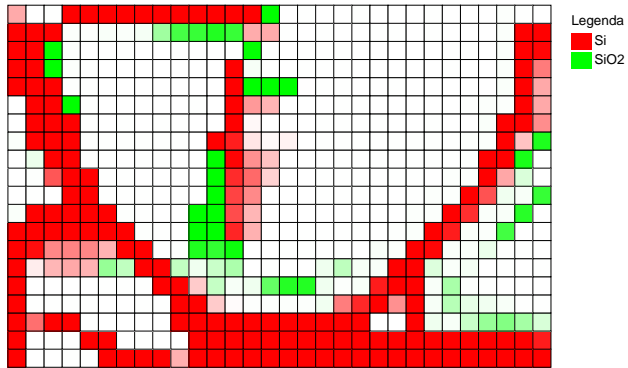

(b) 150 iterações

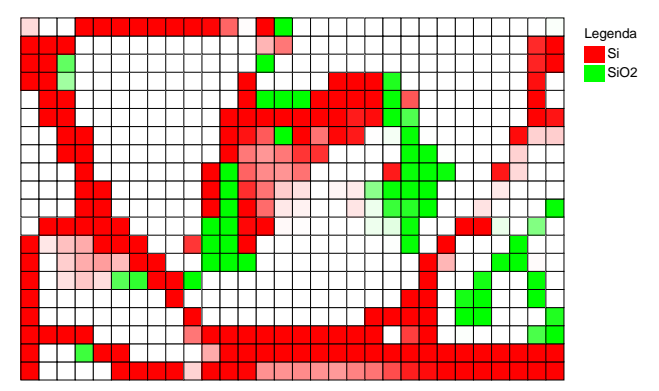

(d) 300 iterações

Figura 6.23: Atuador de movimento inverso (com restrições de volume, sem filtro) - Topologias intermediárias (vermelho - Si; verde - $\mathrm{SiO}_{2}$ ).

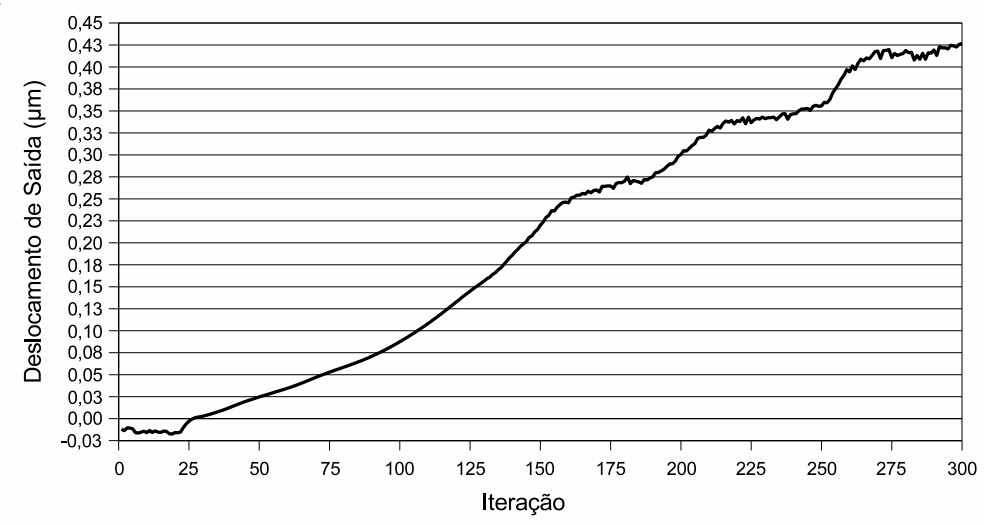

Figura 6.24: Atuador de movimento inverso (com restrições de volume, sem filtro) - Função Objetivo. 
ação independe da discretização), controla também a complexidade da topologia final, permitindo resultados mais simples e passíveis de serem fabricados. No trabalho de Yin e Ananthasuresh (2002), ao considerar a convecção lateral dos elementos e ter como função objetivo o deslocamento de saída, a otimização tende a gerar estruturas menos complexas e mais coesas (com menos furos), de modo a minimizar a perda de calor por conveç̧ão lateral e maximizar a expansão térmica da estrutura. Tendo isso em mente, a implementação do filtro no presente trabalho teve papel importante na reprodução do resultado da literatura.

Outra influência importante do uso do filtro espacial é o de favorecer o aparecimento de materiais intermediários fictícios (as escalas de cinza) ao limitar o gradiente de material. Para eliminar parte desses materiais indesejados, o filtro é desativado em certo ponto para que a otimização possa reduzir essas ocorrências. Quando o filtro é desativado por muitas iterações, a topologia tende a adquirir características da obtida sem o uso do filtro espacial. Pode-se chegar a essa conclusão verificando as semelhanças entre a figura $6.25(\mathrm{~d})$, cuja topologia foi obtida com a desativação do filtro nas 200 iterações que antecederam o resultado final, e a figura 6.23(d), obtida totalmente sem o uso do filtro.

\subsubsection{Coeficiente de Continuação dos Desvios Padrão}

Para estudar os efeitos dos coeficientes de continuação $\omega_{D P_{m}}$, foram realizadas duas otimizações utilizando a metade e o dobro do valor dos $\omega_{D P_{m}}$ usado na seção 6.1. O número total de iterações e o número da iteração onde o filtro é desativado foram alterados para cada caso, para que os desvios padrão fossem aproximadamente os mesmos para o início, meio e fim das otimizações se comparados com o resultado original. Assim, uma otimização foi realizada com um máximo de 600 iterações (300 sem uso do filtro espacial) e $\omega_{D P_{m}}=0,005$, obtendo a topologia ótima próximo à iteração 500. A outra foi feita com 150 iterações (75 sem uso do filtro espacial) e $\omega_{D P_{m}}=0,02$, com topologia ótima na iteração 115. Os resultados estão apresentados da figura 6.25 a 6.28 .

No caso de $\omega_{D P_{m}}=0,005$, uma redução do coeficiente de continuação tornou necessário um aumento do número de iterações, ou seja, o custo computacional. Por outro lado, isso favoreceu a atenuação de estruturas espúrias, mas também degradou a solução devido ao grande número de iterações sem o uso do filtro, conforme discutido na seção anterior. Reduzir o número de iterações finais sem o filtro melhoraria sensivelmente o resultado.

Utilizando $\omega_{D P_{m}}=0,02$, tivemos o inverso: o pouco número de iterações 


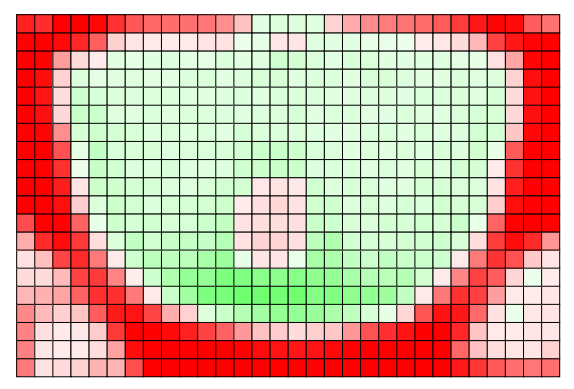

(a) 125 iterações

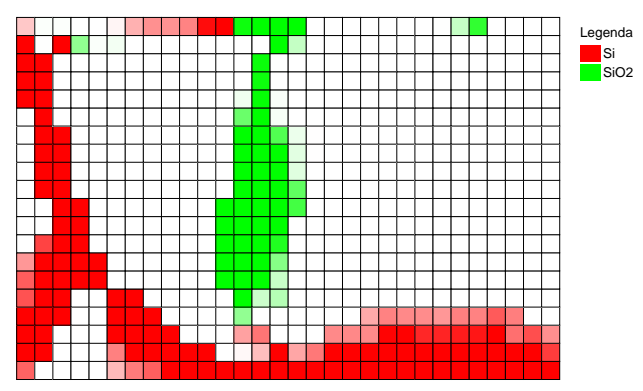

(c) 375 iterações

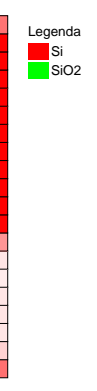

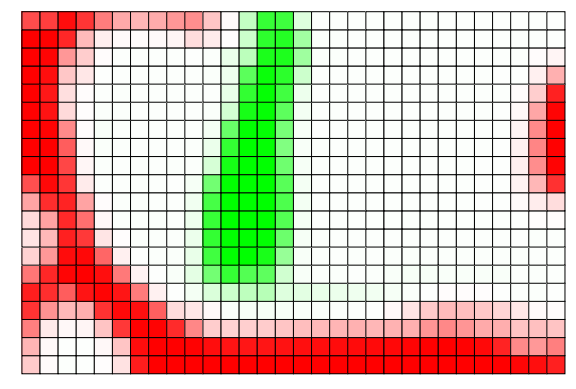

(b) 250 iterações

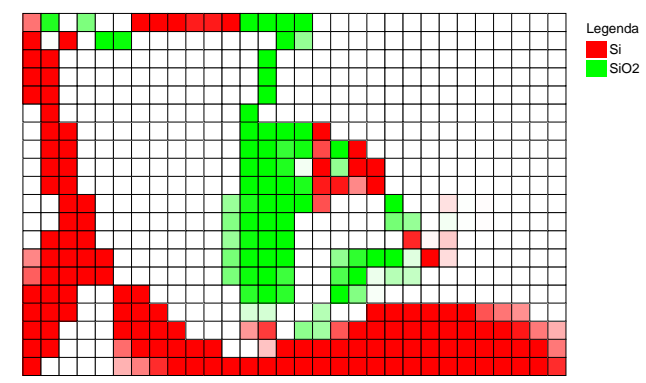

(d) 500 iterações

Figura 6.25: Atuador de movimento inverso (com restrições de volume, $\omega_{D P_{m}}=0,005(m=1$ e 2$\left.)\right)$ - Topologias intermediárias (vermelho - Si; verde $\mathrm{SiO}_{2}$ ).

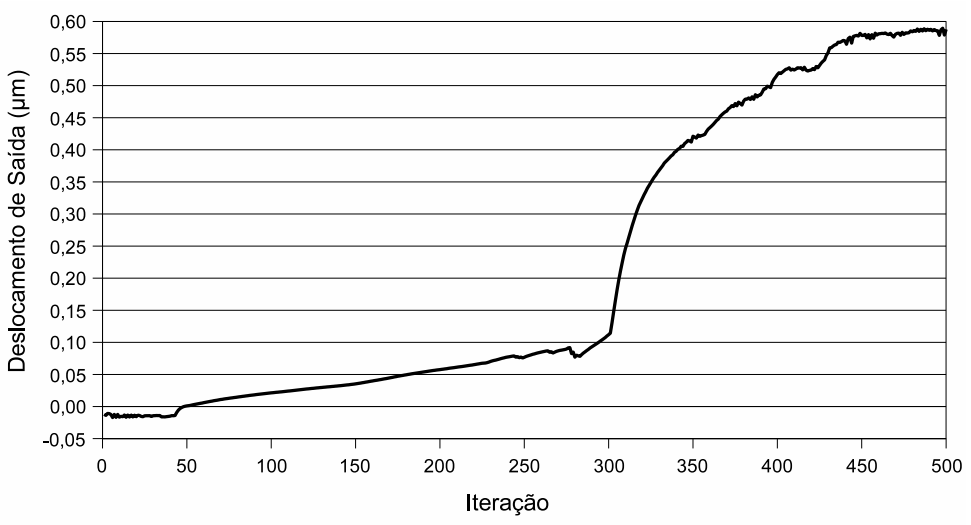

Figura 6.26: Atuador de movimento inverso (com restrições de volume, $\omega_{D P_{m}}=0,005(m=1$ e 2$\left.)\right)$ - Função Objetivo. 


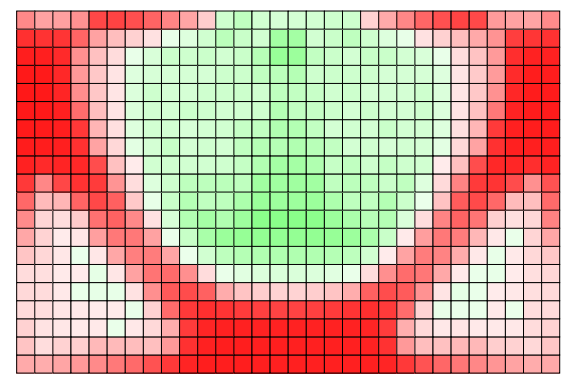

(a) 30 iterações

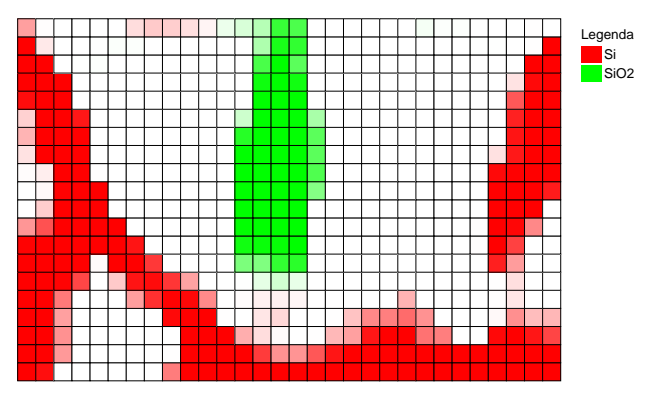

(c) 90 iterações

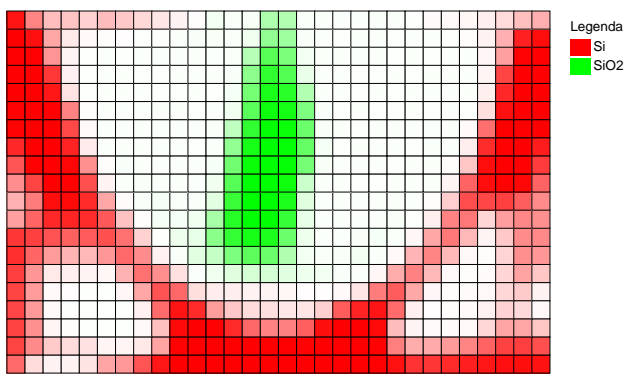

(b) 60 iterações

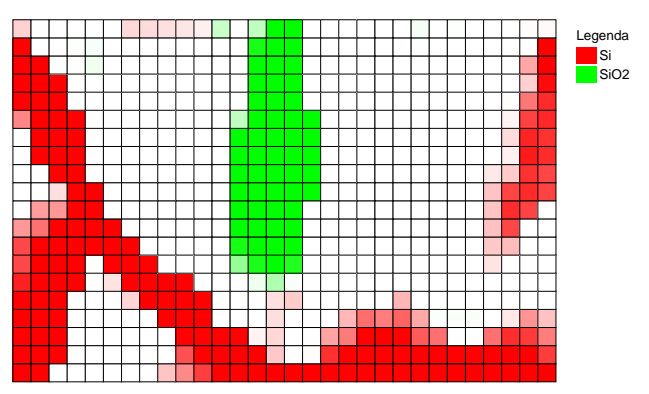

(d) 115 iterações

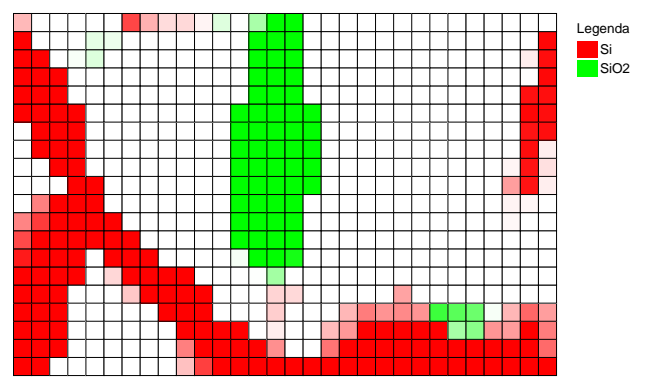

(e) 150 iterações

Figura 6.27: Atuador de movimento inverso (com restrições de volume, $\omega_{D P_{m}}=0,02(m=1$ e 2$\left.)\right)$ - Topologias intermediárias (vermelho - Si; verde $\left.\mathrm{SiO}_{2}\right)$.

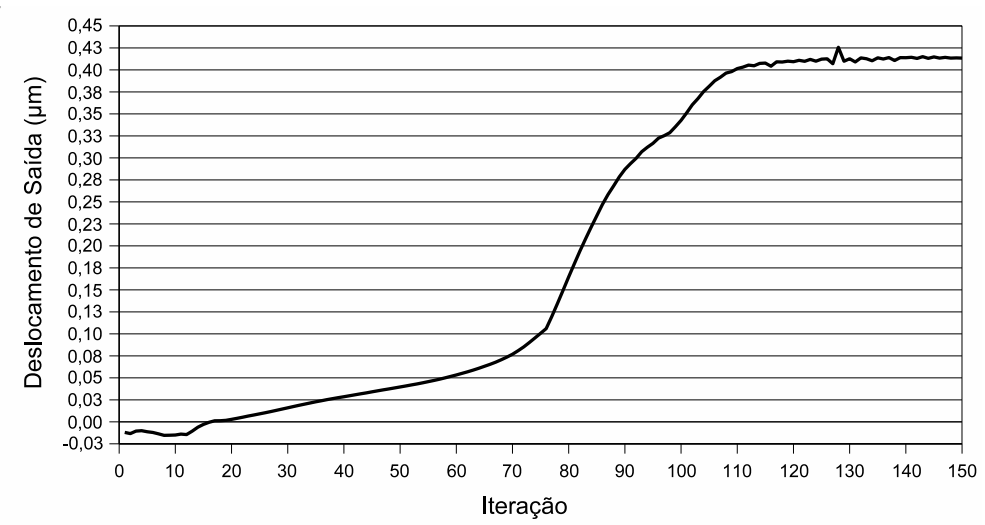

Figura 6.28: Atuador de movimento inverso (com restrições de volume, $\omega_{D P_{m}}=0,02(m=1$ e 2$\left.)\right)-$ Função Objetivo. 


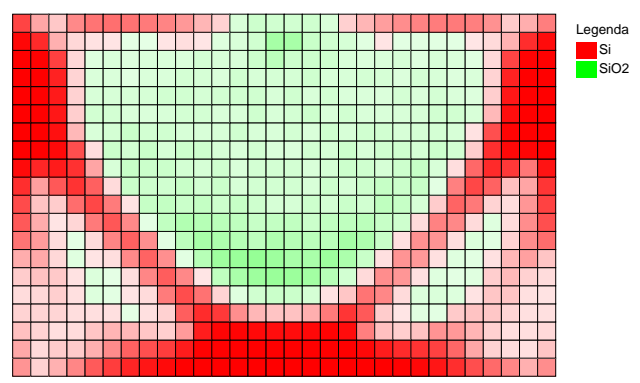

(a) 50 iterações

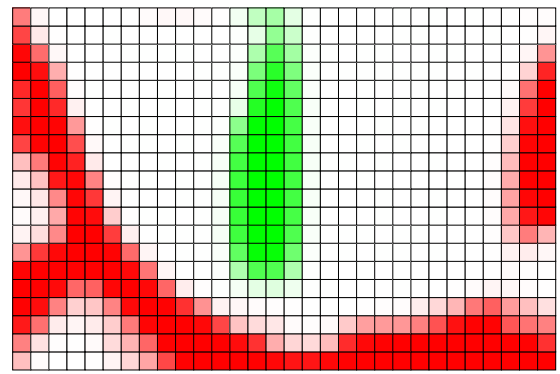

(c) 150 iterações

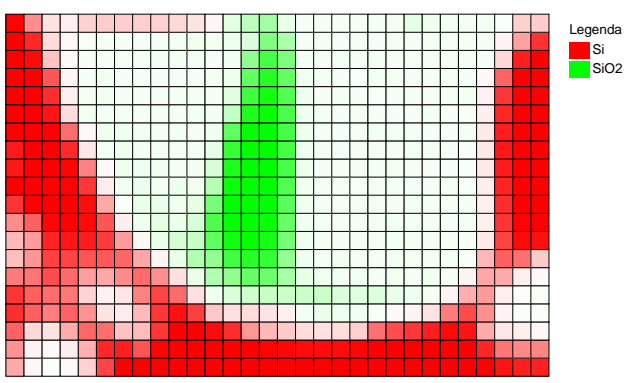

(b) 100 iterações

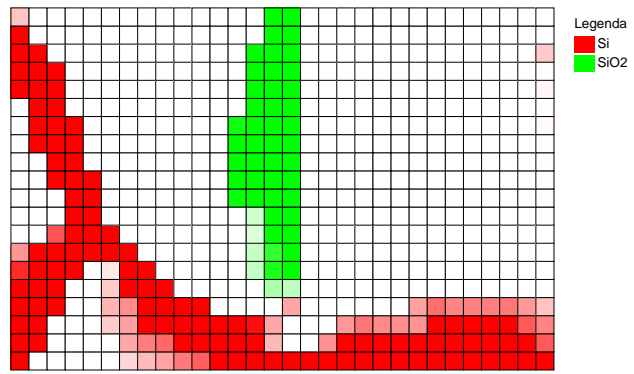

(d) 200 iterações

Figura 6.29: Atuador de movimento inverso (com restrições de volume, função de pico modificada) - Topologias intermediárias (vermelho - Si; verde - $\mathrm{SiO}_{2}$ ).

não permitiu que as estruturas espúrias desaparecessem por completo ao atingir a topologia ótima. Até o final das 150 iterações, ainda existiam estruturas desconexas, que possivelmente desapareceriam em futuras iterações. Uma possível solução seria aumentar o passo dos limites móveis, sob o risco de torná-lo grande o suficiente para degradar a solução devido à utilização das aproximações lineares da PLS.

\subsubsection{Parâmetros da Função de Pico - Média e Desvio Padrão}

Para os resultados da otimização do atuador de movimento inverso (seção 6.1), as médias $\mu_{m}$ e os desvios padrão $\sigma_{m}$ iniciais foram fixados e usados em todos os resultados até aqui apresentados: $\mu_{m=1}=1,0 ; \mu_{m=2}=2,0 ; \sigma_{m=1}=\sigma_{m=2}=0,3$. Os valores das médias não influenciam os resultados da otimização, dado que são arbitrários. A separação entre as médias não influencia o resultado da otimização desde que os desvios padrão sejam alterados proporcionalmente. Os resultados da otimização do atuador de movimento inverso, com restrições de volume, foram obtidos novamente utilizando uma função de pico com médias $\mu_{m=1}=0,5$ e $\mu_{m=2}$ $=1,0$. Os desvios iniciais foram $\sigma_{m=1}=\sigma_{m=2}=0,15$ e o desvio foi limitado a um mínimo de 0,05. As figuras 6.29 e 6.30 mostram os resultados obtidos. 


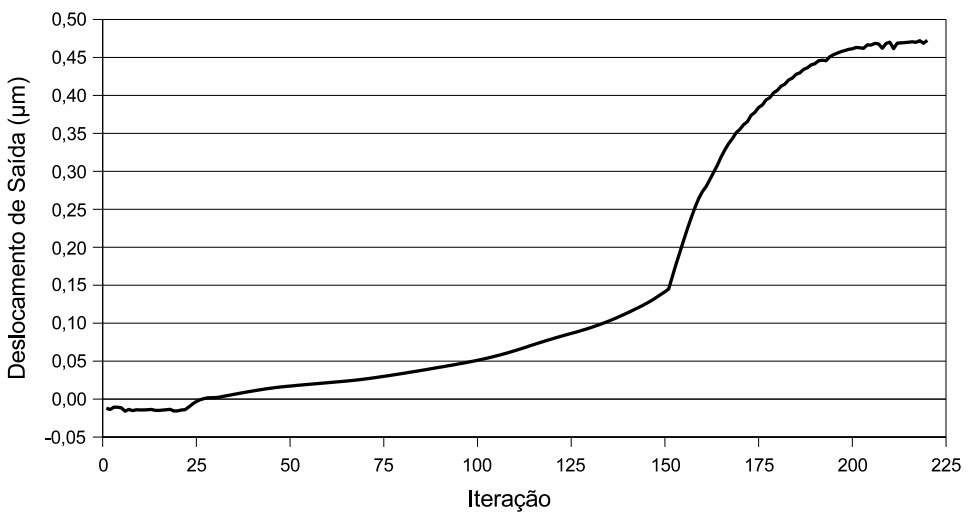

Figura 6.30: Atuador de movimento inverso (com restrições de volume, função de pico modificada) - Função Objetivo.

As topologias intermediárias e curvas da função objetivo obtidas são exatamente as apresentadas nas figuras 6.6 e 6.7 .

\subsection{Atuador multiflexível}

No mecanismo do atuador multiflexível em questão, o objetivo é fazer com que uma peça seja atuada nas direções $x$ ou $y$ de acordo com a atuação elétrica do mecanismo. Foi utilizado um domínio fixo estendido com dimensões $3 \mathrm{~mm} \times 2 \mathrm{~mm}$, discretizado em $30 \times 20$ elementos, e espessura de $15 \times 10^{-3} \mathrm{~mm}$. Os nós de dois terços da parte superior e inferior do domínio foram restritos em $x$ e $y$ e com temperatura ambiente $T_{a m b}$. Os nós da parte superior com restrição foram eletricamente aterrados. Os nós da parte inferior com restrição foram usados de acordo com os casos de carga: no caso de carga 1, foi imposta uma tensão elétrica de $0,1 \mathrm{~V}$ sobre a primeira metade dos nós; no caso de carga 2, uma tensão elétrica de mesma magnitude foi imposta sobre a segunda metade. Sobre toda a face do mecanismo, foi considerado um coeficiente de convecção $h_{0}=2,5 \times 10^{-4} \mathrm{~W} / \mathrm{mm}^{2} \cdot \mathrm{K}$. Deseja-se maximizar $u_{\text {saida }}$ e $u_{\text {saida }}$, os deslocamentos de saída para os casos de carga 1 e 2 , respectivamente, onde uma peça de rigidez $K=200 \mathrm{~N} / \mathrm{mm}$ é atuada. A figura 6.31 ilustra o domínio fixo estendido e as condições de contorno aplicadas.

Serão usados até dois materiais na otimização: níquel e cobre. As propriedades de cada um estão relacionadas na tabela 6.7.

O modelo de interpolação de material utilizado foi definido com picos cujas médias podem ser 1,0, representando o Material 1 (Níquel), e 2,0, representando o Material 2 (Cobre). Da mesma maneira que foi feito para o atuador de movimento inverso (seção 6.1), os desvios padrão $\sigma_{m}$ e seus coeficientes de continuação $\omega_{D P_{m}}$ 


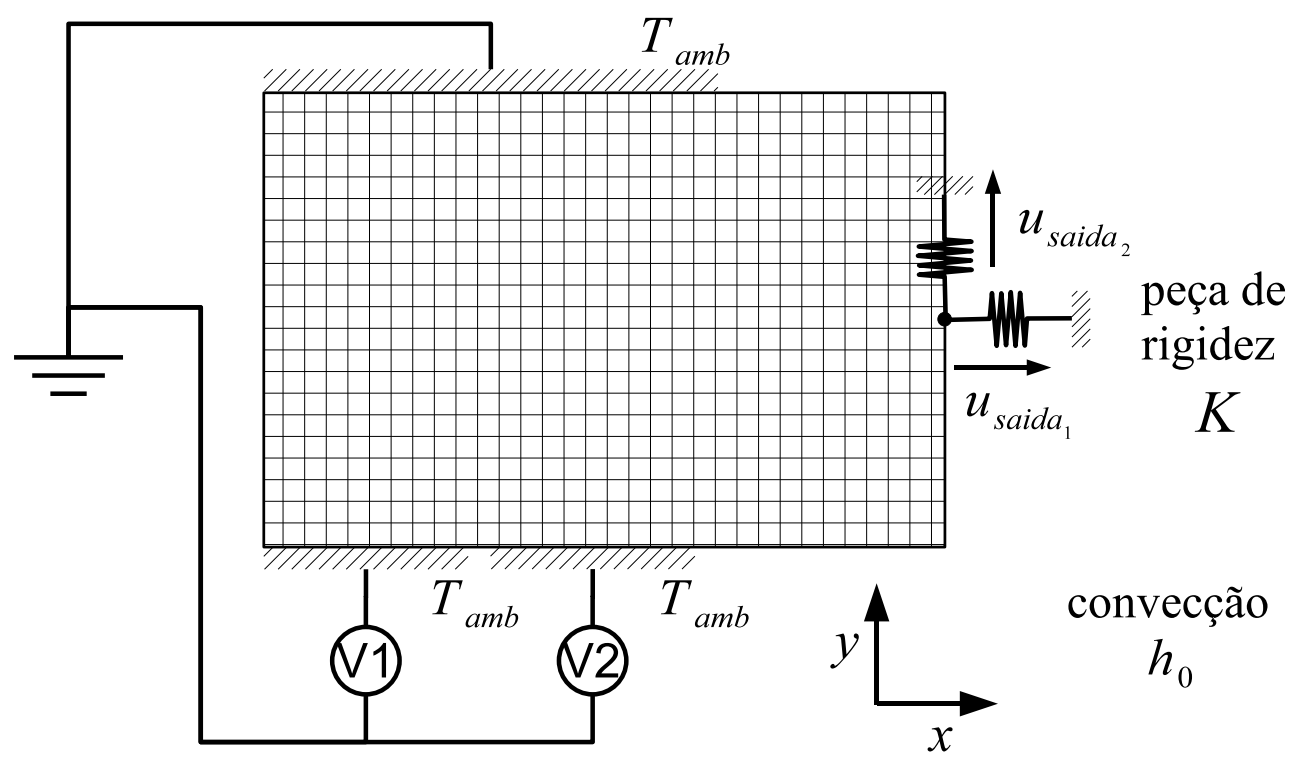

Figura 6.31: Domínio fixo estendido para o atuador multiflexível.

Tabela 6.7: Propriedades dos Materiais - Níquel e Cobre.

\begin{tabular}{|l|c|c|}
\hline Propriedades & Material 1 (Níquel) & Material 2 (Cobre) \\
\hline \hline Módulo de Elasticidade E $\left(\mathrm{N} / \mathrm{mm}^{2}\right)$ & $188 \times 10^{3}$ & $110 \times 10^{3}$ \\
Coeficiente de Poisson & 0,31 & 0,31 \\
Coeficiente de Expansão Térmica $\left(\mathrm{K}^{-1}\right)$ & $15 \times 10^{-6}$ & $16,5 \times 10^{-6}$ \\
Condutividade Elétrica $(1 / \Omega \cdot \mathrm{mm})$ & $6,37 \times 10^{3}$ & $5,977 \times 10^{4}$ \\
Condutividade Térmica $(\mathrm{W} / \mathrm{K} \cdot \mathrm{mm})$ & $90,7 \times 10^{-3}$ & $401 \times 10^{-3}$ \\
\hline
\end{tabular}

(com $m$ denotando o material referido) foram considerados iguais entre si durante a otimização e a continuação se deu de acordo com a equação (5.9). Os $\sigma_{m}$ iniciam o processo de otimização com valor 0,3 , reduzindo-o de acordo com coeficientes $\omega_{D P_{m}}$ de valor igual a 0,01 . O valor mínimo para $\sigma_{m}$ foi de 0,15 por definir bem os picos de cada termo do modelo de material e penalizar menos as densidades intermediárias. Isso poderia facilitar a mudança de material de um elemento.

As pseudo-densidades iniciais foram consideradas uniformes e iguais a 1,5, valor intermediário das posições dos dois picos do modelo de material $\left(\mu_{m}\right.$, para $m=1$ e 2$)$.

O filtro espacial foi utilizado com raio igual a 0,11, de modo a abranger apenas os elementos imediatamente na sua vizinhança na discretização considerada.

O parâmetro de normalização da função $\mathrm{KS}(\psi)$ foi escolhido como $10^{3}$ para que o seu produto com os deslocamentos de saída se aproximasse da unidade, dado que os deslocamentos seriam de grandeza próxima a micrômetros.

Como medida da qualidade da multiflexibilidade, o índice de acoplamento IndAcopl $l_{c}$ de um deslocamento de saída $u_{\text {saidac }_{c}}$ sob um caso de carga $c$ é calculado 
da seguinte forma:

$$
\operatorname{Ind} A \operatorname{copl}_{c}=\frac{u_{\text {saida }_{c}}^{2}}{\sum_{i=1, i \neq c}^{N_{\text {carga }}} u_{\text {saida }_{i}}^{2}}
$$

onde $N_{\text {carga }}$ é o número de casos de carga e $u_{\text {saida }}$ é o deslocamento de saída do caso de carga $i$.

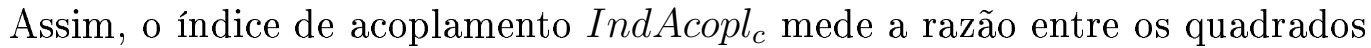
do deslocamento desejado e a soma dos indesejados. Quanto maior IndAcopl $_{c}$, menor o acoplamento entre os deslocamentos de saída sob o caso de carga $c$.

\subsubsection{Uso de dois materiais, além do vazio}

Primeiramente, foram usados ambos os materiais. Foi obtido um mecanismo sem que fossem consideradas restrições de volume dos materiais. Depois, para ter um controle maior sobre o resultado, o volume dos materiais foi restrito.

\section{Sem Restrições de Volume dos Materiais}

Para o caso sem restrições de volume, obteve-se a topologia final após 300 iterações da rotina de otimização e com o filtro espacial desligado nas últimas 20 iterações, consumindo perto de 15 minutos de processamento. A figura 6.32 mostra as topologias intermediárias encontradas até o filtro espacial ser desligado. As curvas da função objetivo e deslocamentos de saída, do acoplamento entre as saídas para cada caso de carga e dos volumes relativos dos materiais são apresentadas na figura 6.33.

Após a desativação do filtro na iteração 280, a função objetivo aumenta rapidamente, porém não há a preocupação em se obter uma convergência a um valor, porque, para isso, um número maior de iterações sem o filtro seria requerida e os problemas atenuados pelo uso do filtro tenderiam a retornar, como a dependência da resposta em relação à malha e a instabilidade de tabuleiro. Esta última começou a aparecer de forma acentuada após as 20 iterações finais em que o filtro permaneceu desativado. A interface entre as estruturas em níquel e cobre consiste de elementos com escala de cinza, que devem ser interpretados para tornar possível sua fabricação.

Sobre a topologia resultante, foi feita uma interpretação de modo a tornála factível: os elementos com mais de $50 \%$ do material predominante tiveram a pseudo-densidade alterada para 100\% desse mesmo material; caso contrário, recebe o valor do vazio para a pseudo-densidade. Além dessa interpretação, 


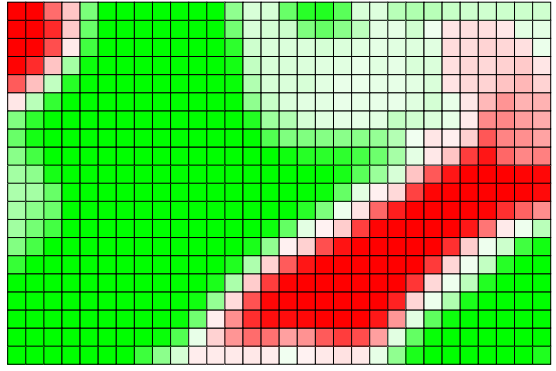

(a) 75 iterações

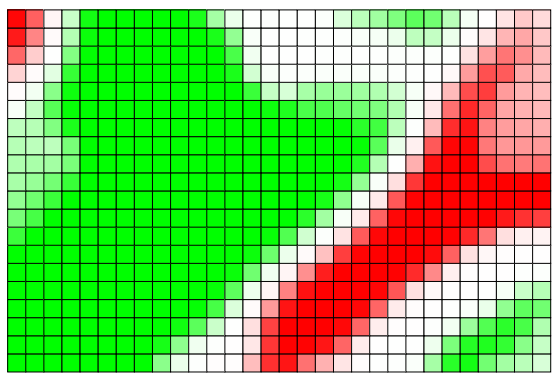

(c) 225 iterações

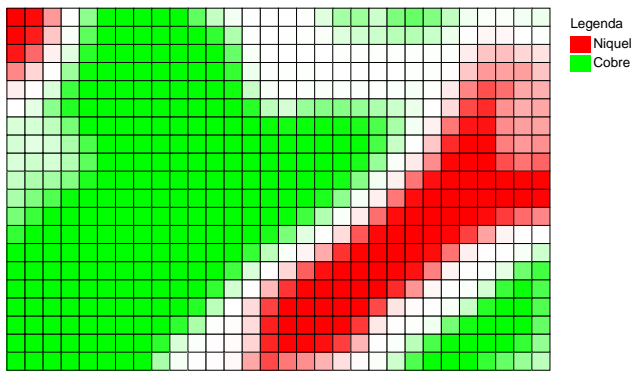

(b) 150 iterações

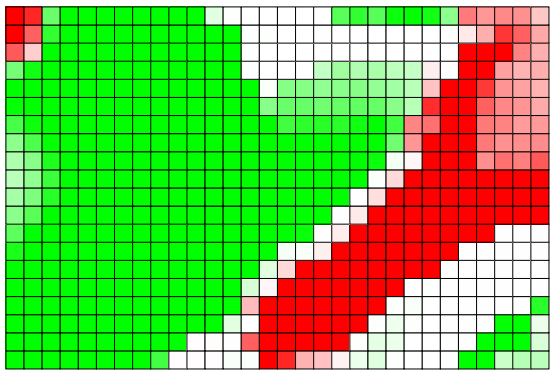

(d) 300 iterações

Figura 6.32: Atuador multiflexível - Topologias intermediárias (vermelho - Ni; verde - $\mathrm{Cu}$ ).

alguns elementos em locais estratégicos (como os pontos restritos em $x$ e $y$ e com tensão elétrica aplicada, além da ligação entre as estruturas em níquel e em cobre) foram fixos num determinado material, independente do valor da pseudodensidade resultante da otimização. A topologia obtida e suas análises pelo MEF estão apresentadas nas figuras 6.34 e 6.35. As características desta topologia são mostradas na tabela 6.8 .

Tabela 6.8: Atuador multiflexível - Características da topologia interpretada.

\begin{tabular}{|l|c|}
\hline Característica & Valor \\
\hline \hline Caso de Carga 1 & \\
- Deslocamento de Saída & $3,033 \mu \mathrm{m}$ \\
- Indice de Acoplamento & $8,876 \times 10^{1}$ \\
& \\
Caso de Carga 2 & \\
- Deslocamento de Saída & $6,822 \times 10^{-1} \mu \mathrm{m}$ \\
- Índice de Acoplamento & $4,386 \times 10^{-1}$ \\
& \\
Volumes Relativos & \\
- Níquel & $27,59 \%$ \\
- Cobre & $31,77 \%$ \\
- Total & $\mathbf{5 9 , 3 6 \%}$ \\
\hline
\end{tabular}

A topologia obtida tem apenas o deslocamento de saída do caso de carga 1 com pouco acoplamento. O deslocamento do caso de carga 2 é menor que 


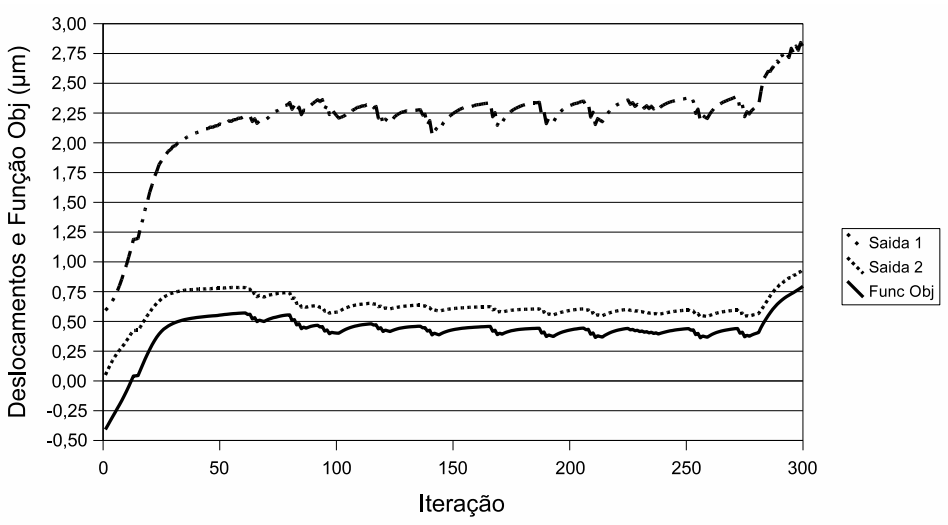

(a) Deslocamentos de saída e Função objetivo

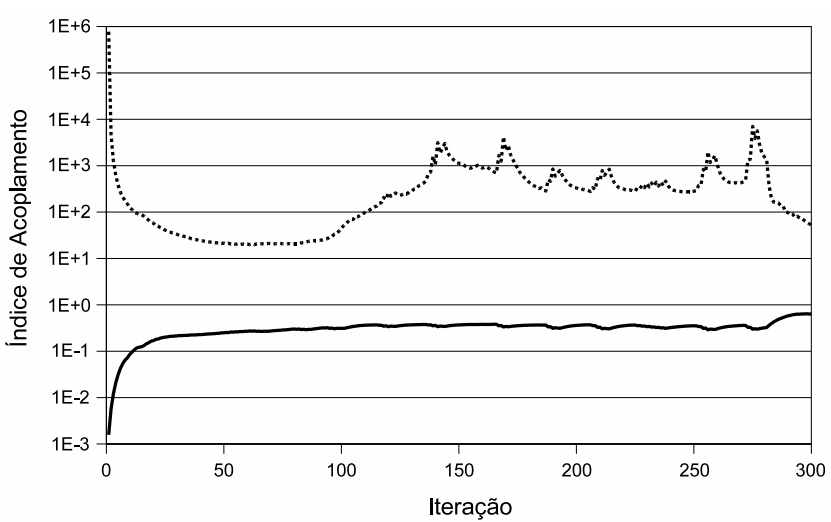

(b) Acoplamentos dos deslocamentos de saída

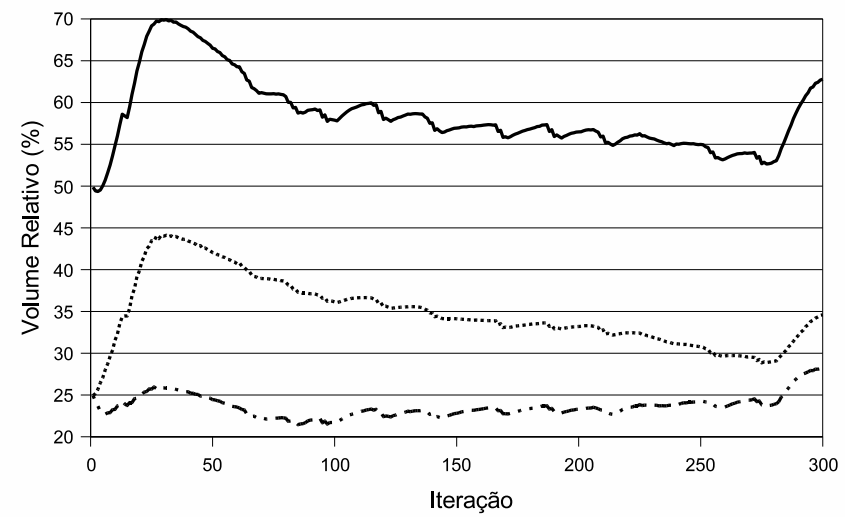

(c) Volumes relativos dos materiais

Figura 6.33: Atuador multiflexível - Curvas da otimização. 


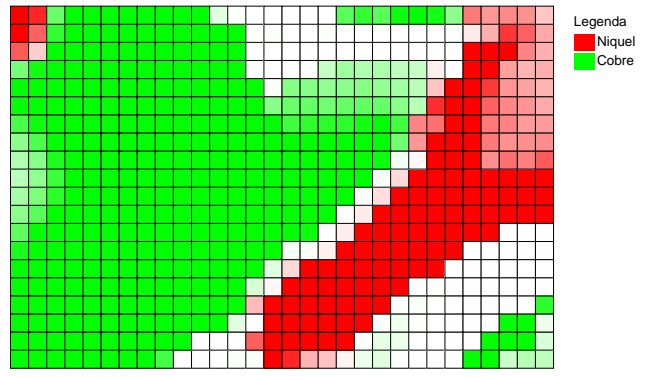

(a) Topologia resultante (vermelho - Ni; verde $-\mathrm{Cu})$
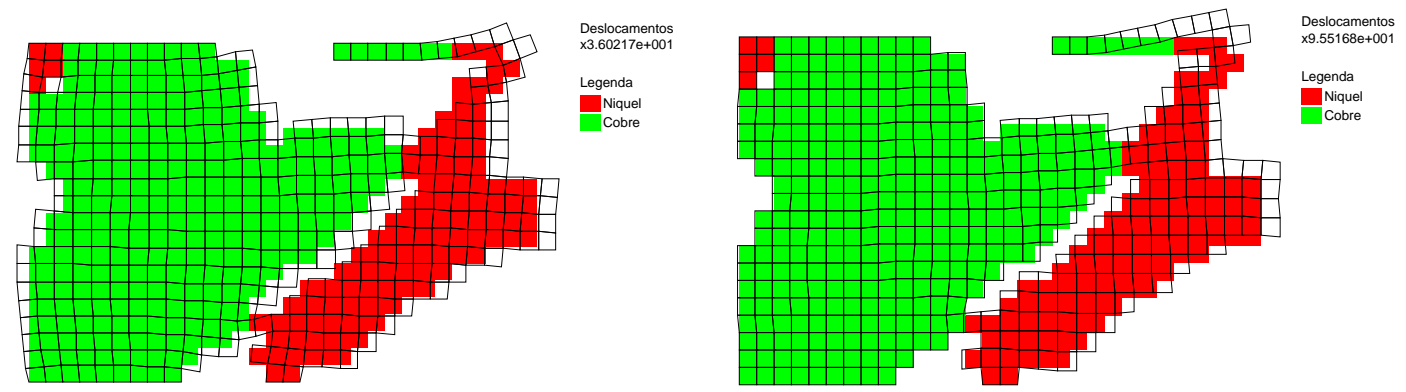

(b) Topologia interpretada e Deslocamentos (c) Topologia interpretada e Deslocamentos (Caso de Carga 1) (Caso de Carga 2)
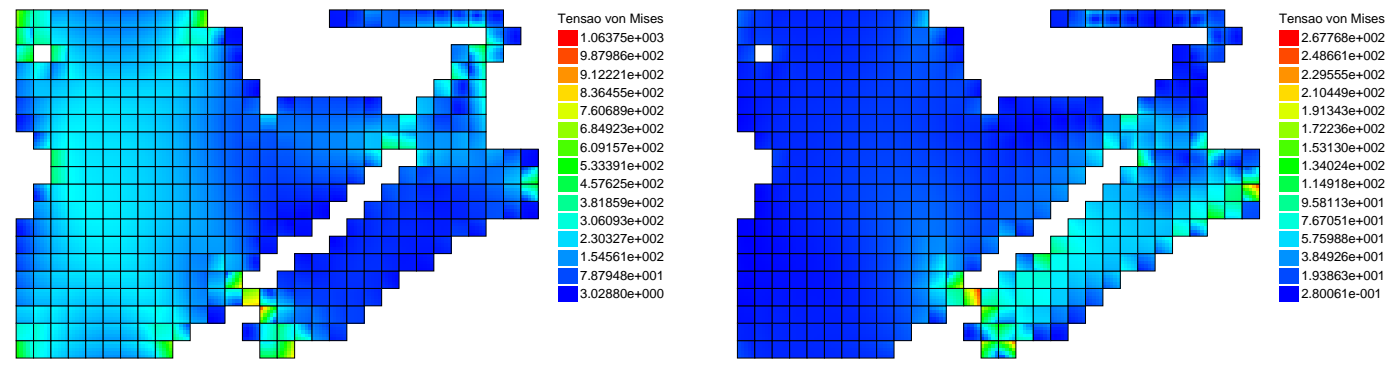

(d) Tensões de von Mises (Caso de Carga 1)

(e) Tensões de von Mises (Caso de Carga 2)

Figura 6.34: Atuador multiflexível - Topologia final e interpretada, Análise termoelástica. 

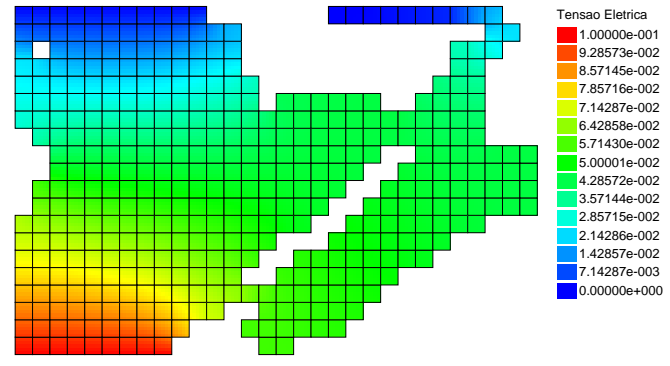

(a) Tensões Elétricas (Caso de Carga 1)
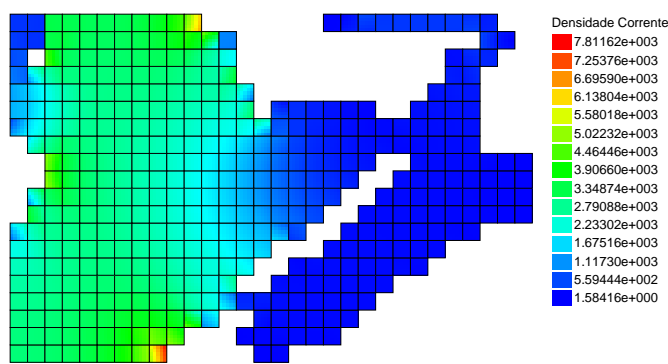

(c) Densidades de Corrente (Caso de Carga 1)
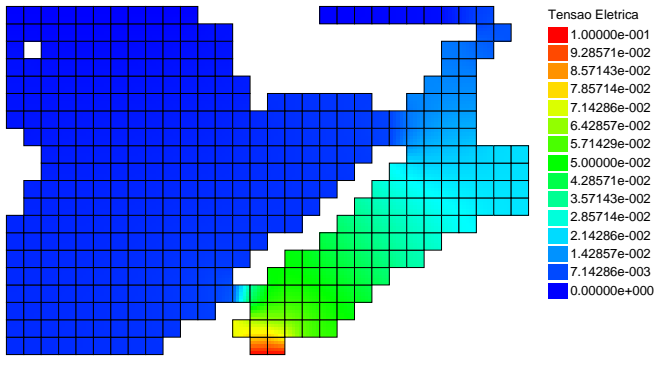

(b) Tensões Elétricas (Caso de Carga 2)
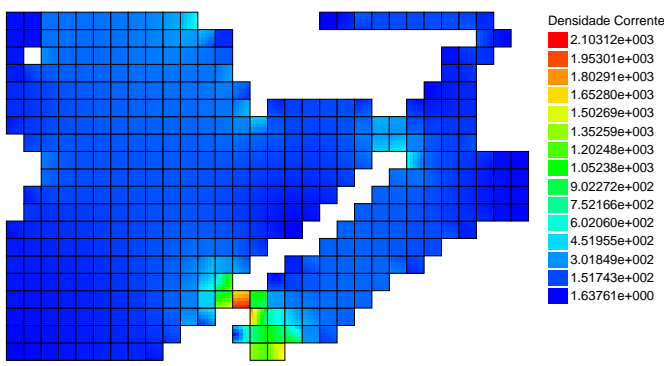

(d) Densidades de Corrente (Caso de Carga 2)
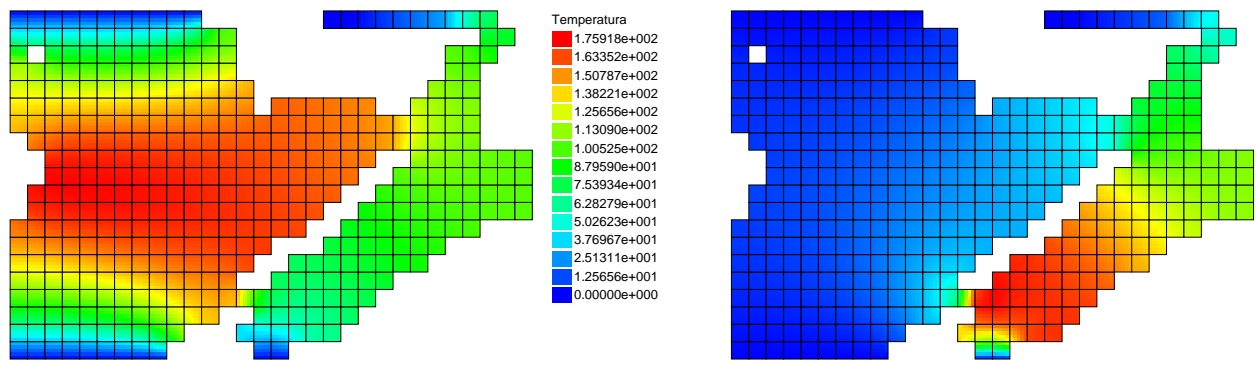

Temperatura $8.09202+001$
$7.46955+00$ $6.84709 \mathrm{e}+00$ $5.60217 \mathrm{e}+001$ $-4.95724 \mathrm{e}+001$ $3.73478 \mathrm{e}+00$

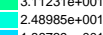

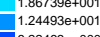
$6.22463 \mathrm{e}+000$

(e) Variações de Temperatura (Caso de Carga (f) Variações de Temperatura (Caso de Carga 1) 2)

Figura 6.35: Atuador multiflexível - Análise elétrica e eletrotérmica. 


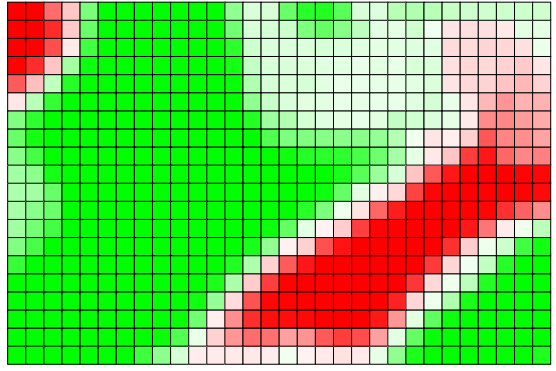

(a) 75 iterações

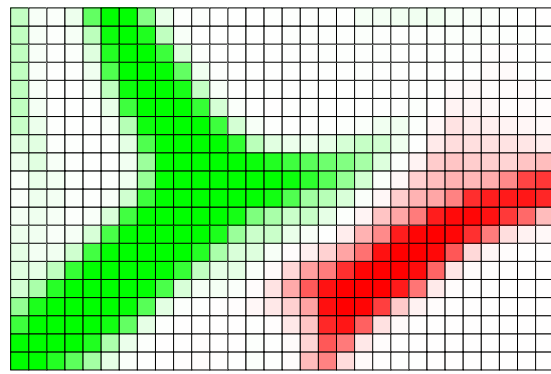

(c) 225 iterações

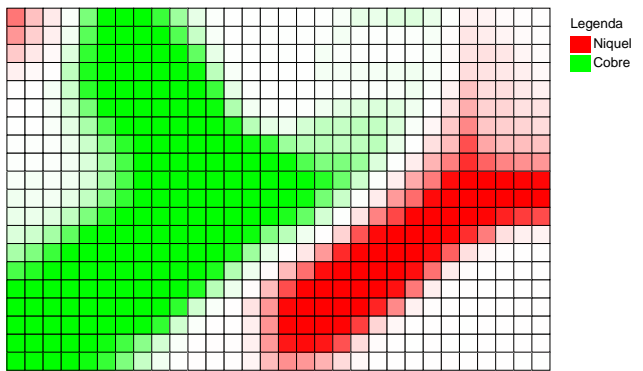

(b) 150 iterações

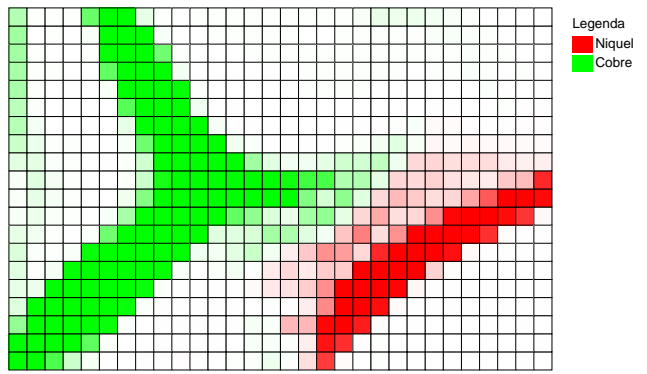

(d) 290 iterações

Figura 6.36: Atuador multiflexível (com restrições de volume) - Topologias intermediárias (vermelho - $\mathrm{Ni}$; verde - $\mathrm{Cu}$ ).

o movimento acoplado (o deslocamento do grau de liberdade de saída para o caso de carga 1), fato demonstrado pelo índice de acoplamento menor que 1. Apesar do forte acoplamento para o caso de carga 2, pode-se verificar que a atuação eletrotérmica acontece em parte diferentes (figura 6.35), assim como os movimentos resultantes de cada caso de carga são distintos (figura 6.34), o que caracterizaria a multiflexibilidade do mecanismo.

\section{Com Restrições de Volume dos Materiais}

O próximo resultado foi obtido ao impor restrições de volume de para o níquel e cobre, de $10 \%$ do volume total para cada um, à otimização apresentada. Assim, iniciou-se com volumes máximos de $100 \%$ de volume relativo para cada material, reduzindo-os através da equação (5.10) com um coeficiente de continuação $\omega_{R V_{m}}$ de 0,015. Obteve-se a topologia final após 290 iterações da rotina de otimização, consumindo perto de 15 minutos de processamento. Para reduzir densidades intermediárias e preservar a independência da malha, o filtro espacial foi desligado apenas nas últimas 10 iterações. A figura 6.36 mostra as topologias intermediárias encontradas antes da solução final. As curvas da função objetivo e deslocamentos de saída, do acoplamento entre as saídas para cada caso de carga e dos volumes relativos dos materiais são apresentadas na figura 6.37. 


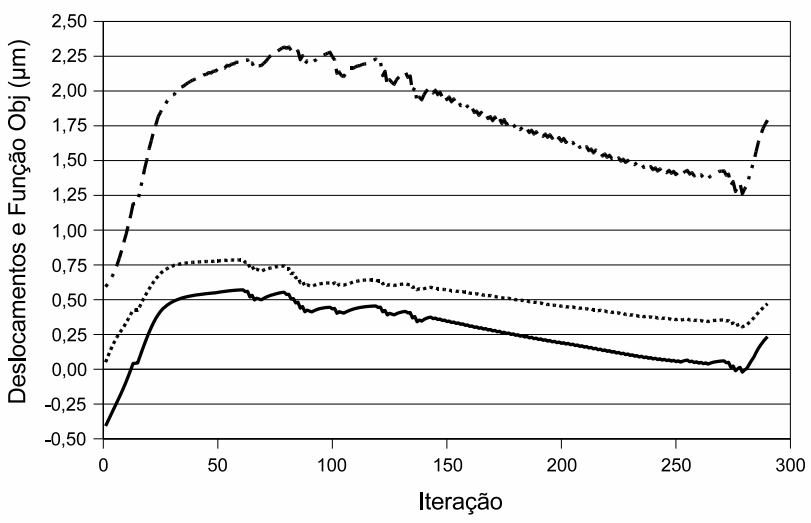

$\because$ Saida 1

$\checkmark$ Func Obj

(a) Deslocamentos de saída e Função objetivo

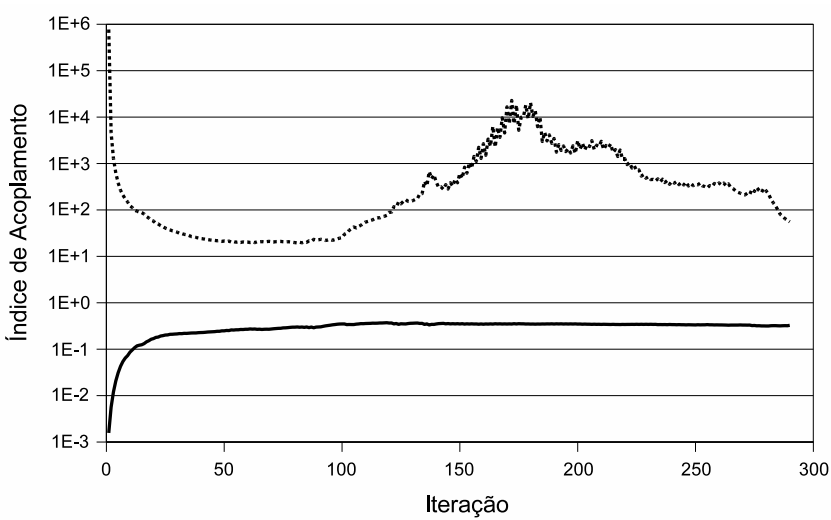

$\because$ Saida 1

(b) Acoplamentos dos deslocamentos de saída

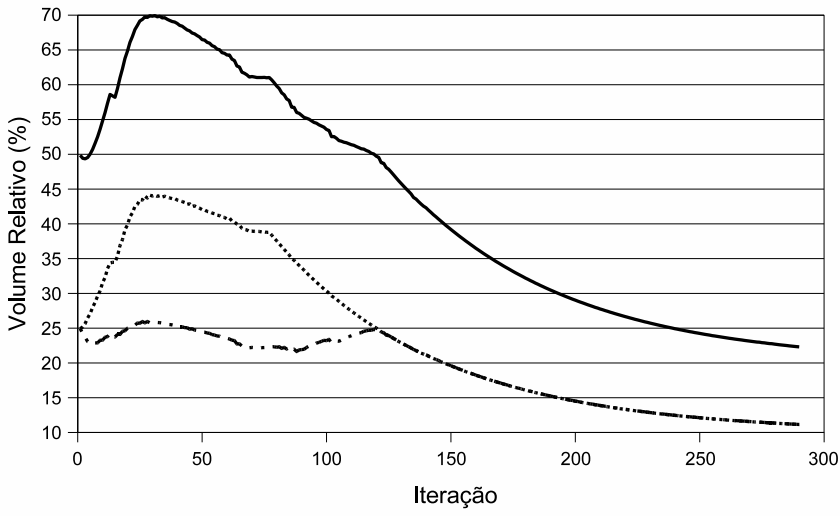

(c) Volumes relativos dos materiais

Figura 6.37: Atuador multiflexível (com restrições de volume) - Curvas da otimização. 


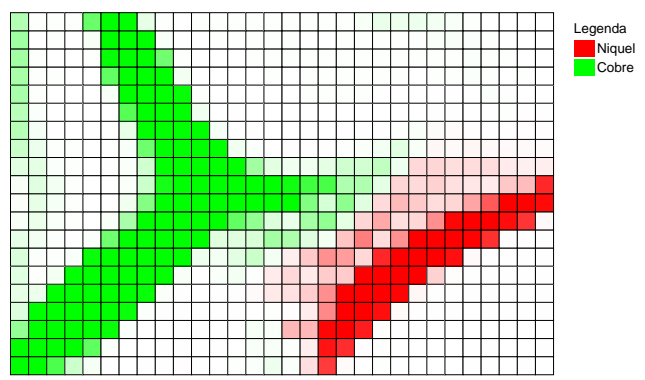

(a) Topologia resultante (vermelho - Ni; verde $-\mathrm{Cu})$
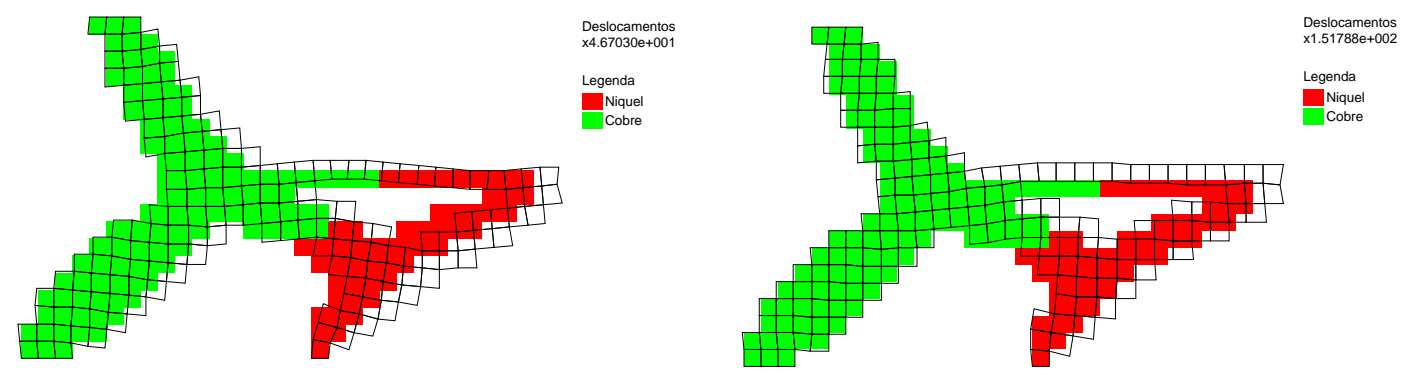

(b) Topologia interpretada e Deslocamentos (c) Topologia interpretada e Deslocamentos (Caso de Carga 1) (Caso de Carga 2)

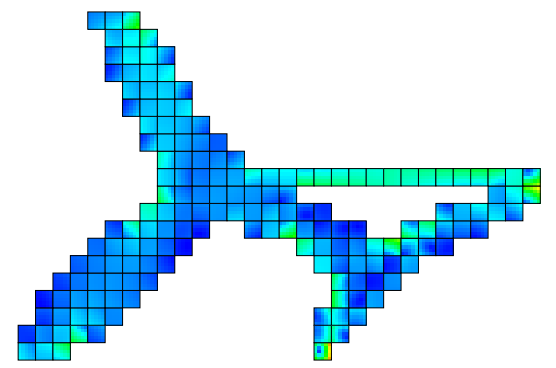

(d) Tensões de von Mises (Caso de Carga 1)
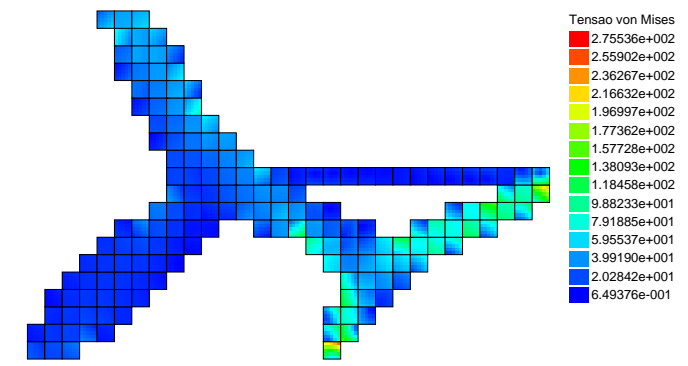

(e) Tensões de von Mises (Caso de Carga 2)

Figura 6.38: Atuador multiflexível (com restrições de volume) - Topologia final e interpretada, Análise termoelástica.

A interface entre as estruturas em níquel e cobre aparece com mais escalas de cinza que no caso sem restrições de material, exigindo uma interpretação mais abrangente em termos de número de elementos cuja densidade foi interpretada. Pela figura 6.37(c), pode-se notar o volume relativo de cada material seguindo a respectiva restrição de volume máximo próximo à iteração 100.

Sobre a topologia resultante, foi feita uma interpretação semelhante ao caso anterior de modo a torná-la factível sem se alterar a sua funcionalidade. A topologia obtida e suas análises pelo MEF estão apresentadas nas figuras 6.38 e 6.39. As características desta topologia são mostradas na tabela 6.9.

A topologia obtida tem um comportamento similar ao resultado da otimização sem restrições de volume de material, com um deslocamento pouco acoplado para o caso de carga 1 e forte acoplamento para o caso de carga 2, como no caso 


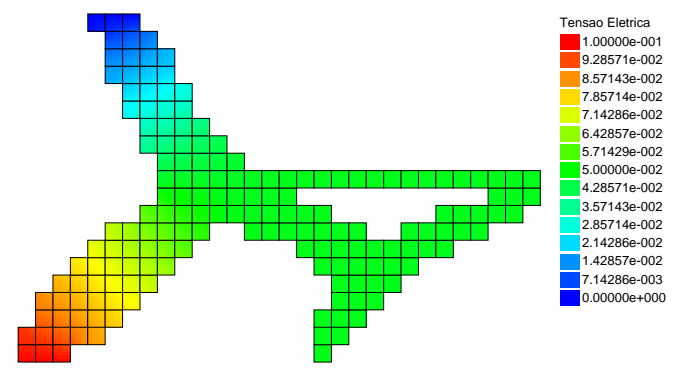

(a) Tensões Elétricas (Caso de Carga 1)
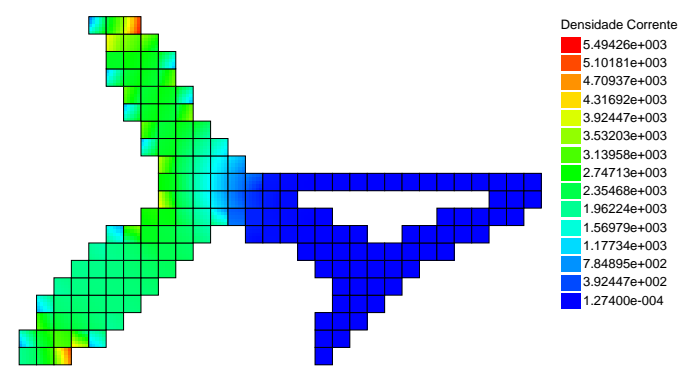

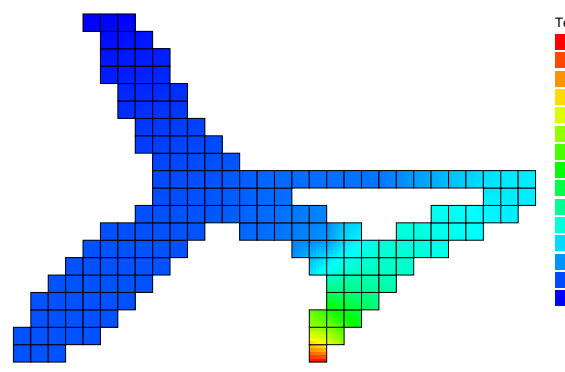

Tensao Eletrica

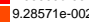

$8.57143 \mathrm{e}-002$

$7.85714 \mathrm{e}-002$
$7.14286 \mathrm{e}-002$

$5.71429 \mathrm{e}-002$

$4.28571 \mathrm{e}-0002$

$3.57143 \mathrm{e}-002$
$2.85714 \mathrm{e}-002$

$2.14286 \mathrm{e}-002$

$1.42857 \mathrm{e}-002$
$7.14286 \mathrm{e}-003$

(b) Tensões Elétricas (Caso de Carga 2)
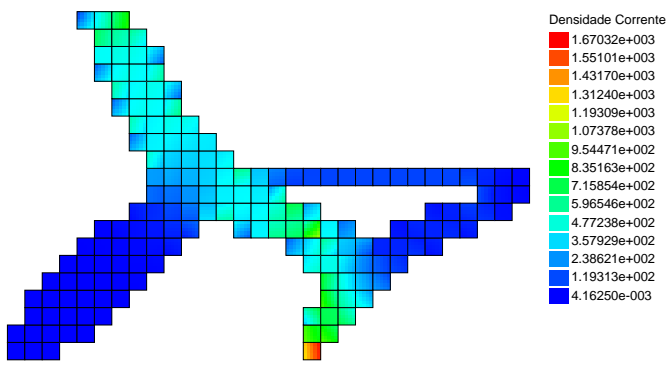

(c) Densidades de Corrente (Caso de Carga 1)

(d) Densidades de Corrente (Caso de Carga 2)
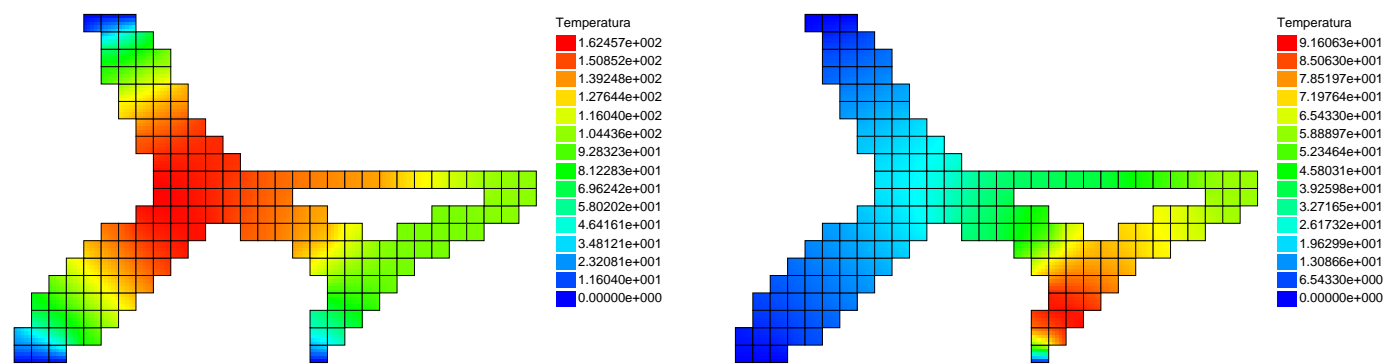

(e) Variações de Temperatura (Caso de Carga (f) Variações de Temperatura (Caso de Carga 1)

2)

Figura 6.39: Atuador multiflexível (com restrições de volume) - Análise elétrica e eletrotérmica. 
Tabela 6.9: Atuador multiflexível (com restrições de volume) - Características da topologia interpretada.

\begin{tabular}{|l|c|}
\hline Característica & Valor \\
\hline \hline Caso de Carga 1 & \\
- Deslocamento de Saída & $3,059 \mu \mathrm{m}$ \\
- Índice de Acoplamento & $7,824 \times 10^{1}$ \\
& \\
Caso de Carga 2 & \\
- Deslocamento de Saída & $4,745 \times 10^{-1} \mu \mathrm{m}$ \\
- Índice de Acoplamento & $1,978 \times 10^{-1}$ \\
& \\
Volumes Relativos & \\
- Níquel & $12,51 \%$ \\
- Cobre & $11,92 \%$ \\
- Total & $\mathbf{2 4 , 4 3 \%}$ \\
\hline
\end{tabular}

anterior. Apesar do acoplamento para o caso de carga 2, também pode-se notar que existe multiflexibilidade, já que as atuações eletrotérmicas e os deslocamentos são distintos para os dois casos de carga considerados. Comparando esse resultado com o mecanismo projetado considerando um único material (seção 6.3.2), houve uma melhora no resultado ao considerar um segundo material na otimização, conforme será visto a seguir.

\subsubsection{Uso de um material, além do vazio}

Visando uma comparação com o projeto que considerou dois materiais, além do vazio, foram obtidos dois atuadores multiflexíveis compostos de um único material: o cobre. Um dos atuadores é resultado da otimização sem restrições de volume dos materiais e o outro, resultado da otimização com estas restrições. O modelo de material continua o mesmo, retirando-se apenas o pico referente ao níquel.

\section{Sem Restrições de Volume dos Materiais}

Inicialmente, não foram impostas restrições de volume dos materiais. Foram registrados os resultados até 300 iterações da rotina de otimização (que consumiram 15 minutos de processamento), desligando-se o filtro espacial nas últimas 20 iterações para que a quantidade de elementos com densidades intermediárias fosse reduzida. O relativamente menor número de iterações sem o filtro nesse caso, se comparado com as otimizações mostradas na seção 6.1 , foi escolhido para que as densidades intermediárias tivessem sua ocorrência reduzida 


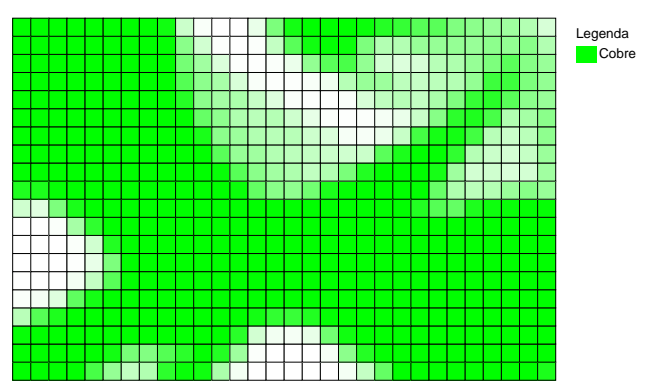

(a) 75 iterações

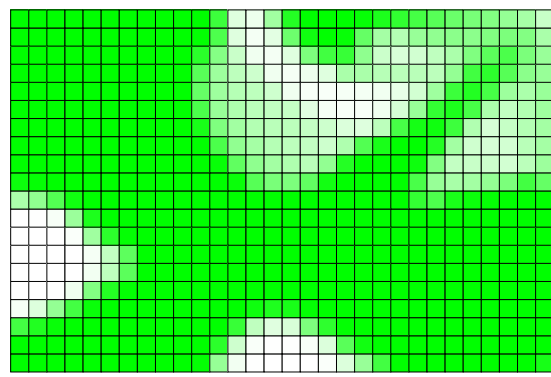

(c) 225 iterações

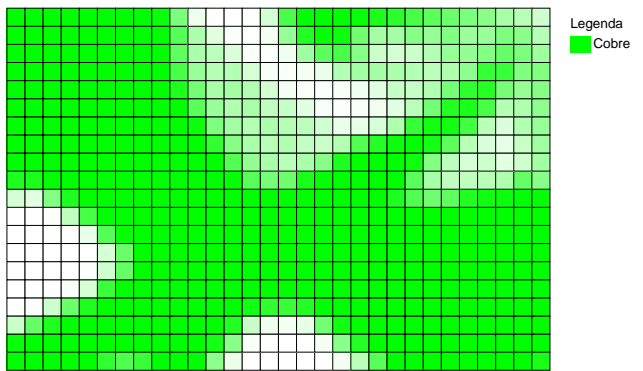

(b) 150 iterações

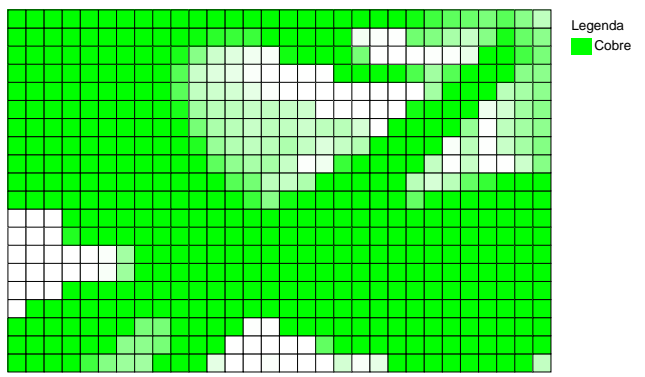

(d) 300 iterações

Figura 6.40: Atuador multiflexível (em cobre) - Topologias intermediárias (vermelho - $\mathrm{Ni}$; verde - $\mathrm{Cu}$ ).

sem, porém, tornar o resultado dependente da malha. A figura 6.40 mostra as topologias intermediárias encontradas antes da solução final. As curvas da função objetivo e deslocamentos de saída, do acoplamento entre as saídas para cada caso de carga e dos volumes relativos dos materiais são apresentadas na figura 6.41.

Sobre a topologia resultante, foi feita uma interpretação de modo a torná-la factível sem se alterar a sua funcionalidade. A topologia obtida e suas análises pelo MEF estão apresentadas nas figuras 6.42 e 6.43. As características desta topologia são mostradas na tabela 6.10 .

Tabela 6.10: Atuador multiflexível (em cobre) - Características da topologia interpretada.

\begin{tabular}{|l|c|}
\hline Característica & Valor \\
\hline \hline Caso de Carga 1 & \\
- Deslocamento de Saída & $3,276 \mu \mathrm{m}$ \\
- Índice de Acoplamento & 6,267 \\
& \\
Caso de Carga 2 & \\
- Deslocamento de Saída & $1,089 \mu \mathrm{m}$ \\
- Índice de Acoplamento & $1,737 \times 10^{-1}$ \\
& \\
Volumes Relativos & $75,30 \%$ \\
- Cobre & $\mathbf{7 5 , 3 0 \%}$ \\
- Total & \\
\hline
\end{tabular}




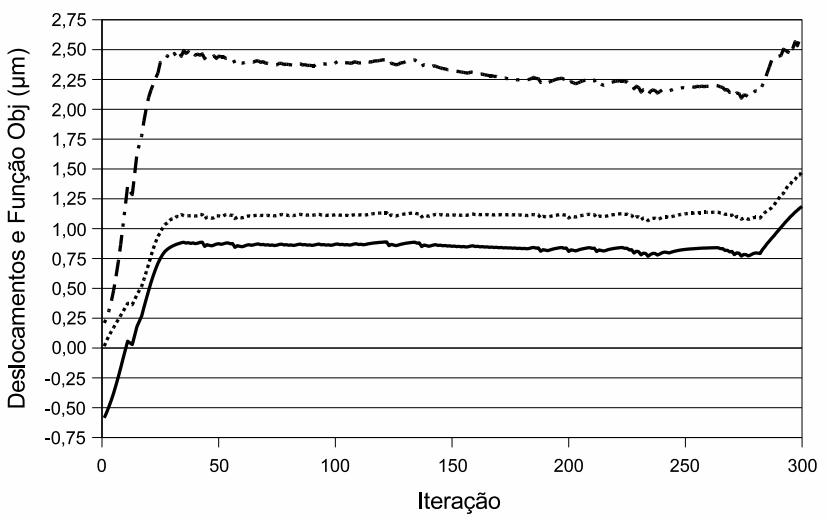

$\because$ Saida 1

$\because$ Func Obj

(a) Deslocamentos de saída e Função objetivo

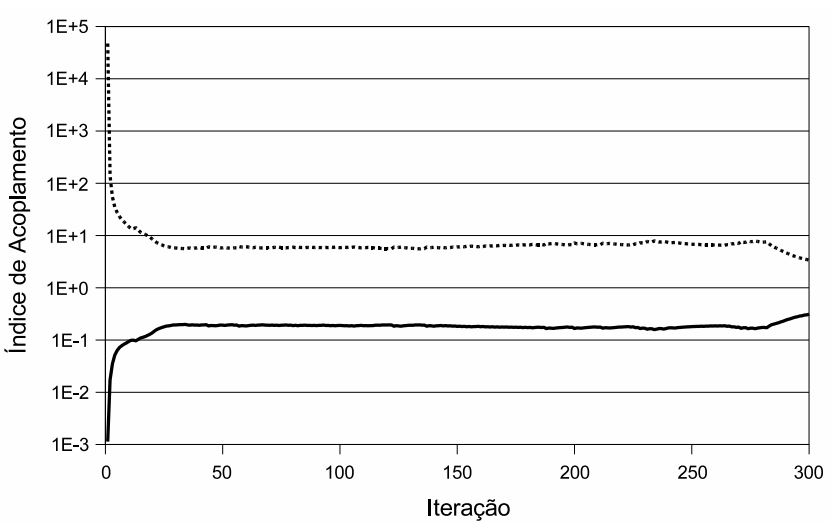

(b) Acoplamentos dos deslocamentos de saída

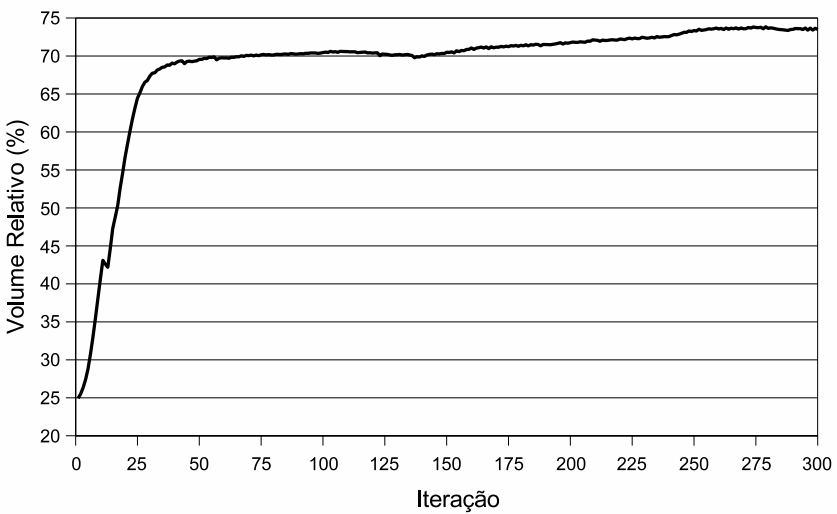

(c) Volumes relativos dos materiais

Figura 6.41: Atuador multiflexível (em cobre) - Curvas da otimização. 


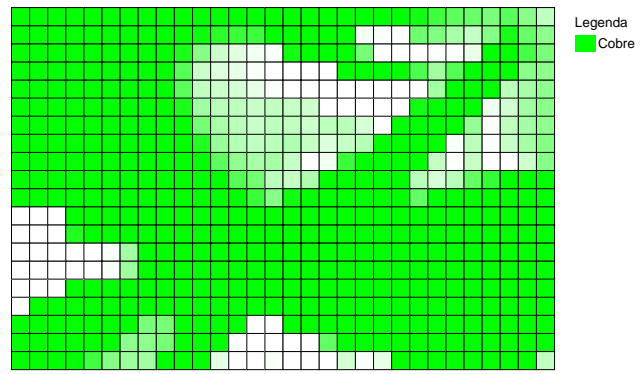

(a) Topologia resultante
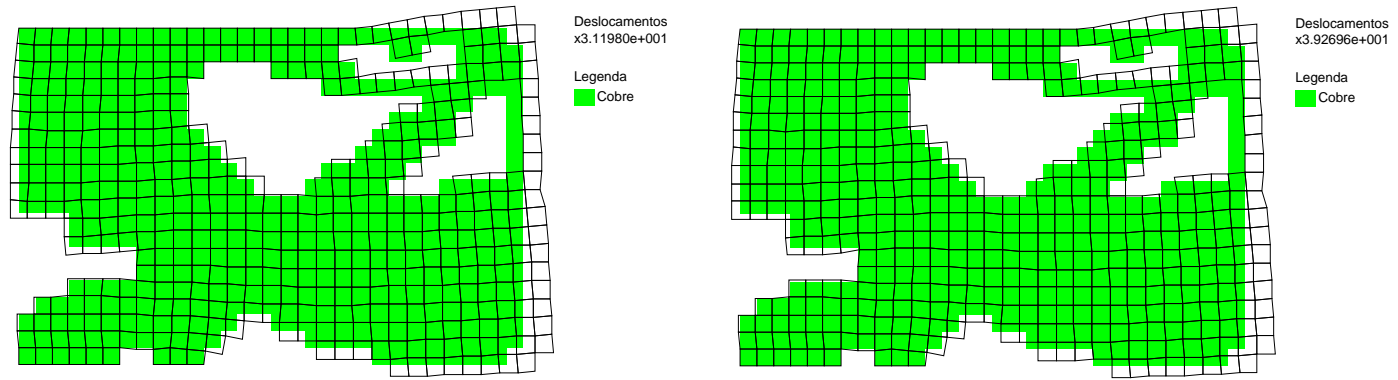

(b) Topologia interpretada e Deslocamentos (c) Topologia interpretada e Deslocamentos (Caso de Carga 1) (Caso de Carga 2)
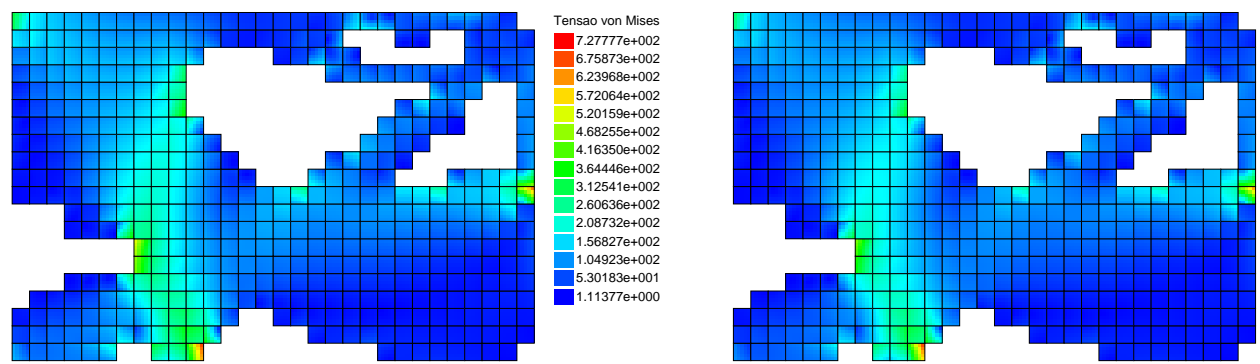

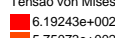

$-5.550738+002$

$4.8673229+002$
$402561+002$

$-3.939390+002$

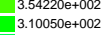

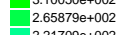

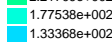

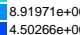

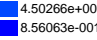

(d) Tensões de von Mises (Caso de Carga 1)

(e) Tensões de von Mises (Caso de Carga 2)

Figura 6.42: Atuador multiflexível (em cobre) - Topologia final e interpretada, Análise termoelástica. 

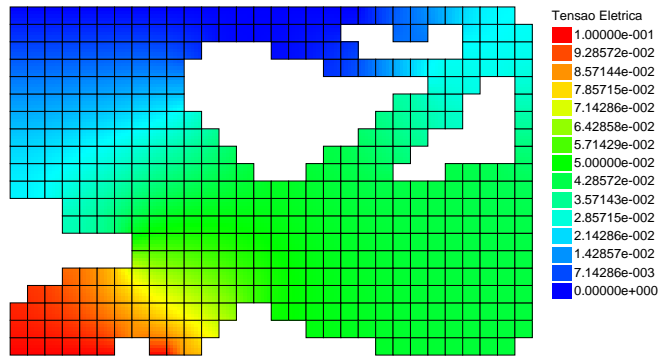

(a) Tensões Elétricas (Caso de Carga 1)
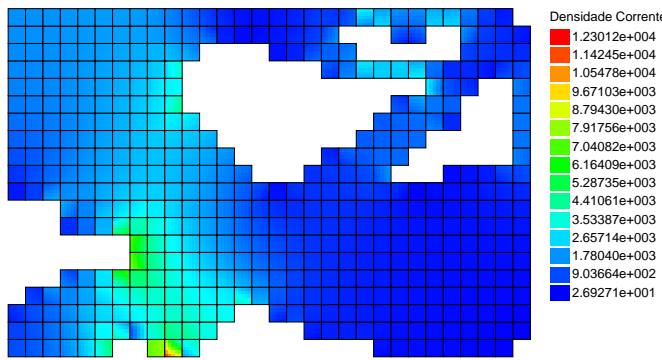

(c) Densidades de Corrente (Caso de Carga 1)
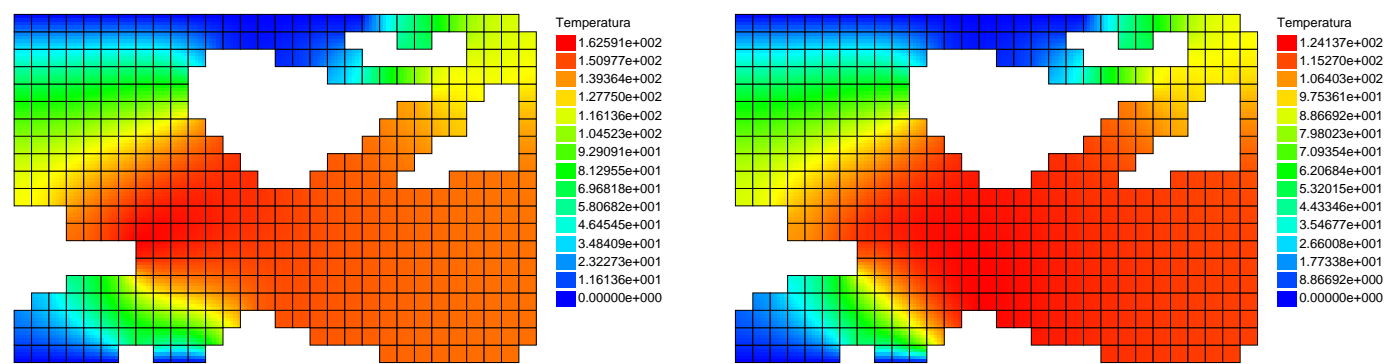

(e) Variações de Temperatura (Caso de Carga (f) Variações de Temperatura (Caso de Carga 1)

Figura 6.43: Atuador multiflexível (em cobre) - Análise elétrica e eletrotérmica. 
A topologia obtida apresenta um mecanismo cujos deslocamentos da estrutura são muito parecidos para ambos os casos de carga. Ao mesmo tempo, o processo de otimização cumpriu seu papel em maximizar o deslocamento das saídas. Assim, pode-se dizer que o resultado é ótimo, mesmo sendo difícil distinguir os resultados de cada caso de carga aplicado. Nesse caso, é até difícil identificar efetivamente uma multiflexibilidade, já que tanto as temperaturas como os deslocamentos são praticamente os mesmos para ambos os casos de carga. Uma implementação de restrições aplicadas aos índices de acoplamento $\operatorname{Ind} A{ }_{c o p l}$, de cada caso de carga $c$, poderia resultar em mecanismos multiflexíveis realmente funcionais, onde cada caso de carga resultaria num deslocamento diferente e facilmente distingüível. Comparando com o projeto de um mecanismo composto por dois materiais, além do vazio (seção 6.3.1), pode-se concluir que a retirada de um dos materiais reduziu a flexibilidade do processo de otimização, o que pode ter contribuído para a obtenção de um resultado menos interessante.

\section{Com Restrições de Volume dos Materiais}

Adicionando uma restrição de volume do cobre ao problema de otimização, o volume máximo se iniciou em $100 \%$ de volume relativo e foi reduzido a $20 \%$ através de um coeficiente de continuação $\omega_{R V_{m}}$ de 0,015 . Essa restrição de volume foi incluída como uma tentativa para se achar um novo máximo local cujos índices de acoplamento fossem melhores. Para este caso, obteve-se a topologia final após 300 iterações da rotina de otimização e com o filtro espacial desligado nas últimas 20 iterações. A figura 6.44 mostra as topologias intermediárias encontradas antes da solução final. As curvas da função objetivo e deslocamentos de saída, do acoplamento entre as saídas para cada caso de carga e dos volumes relativos dos materiais são apresentadas na figura 6.45.

Sobre a topologia resultante, foi feita uma interpretação de modo a tornála factível sem se alterar a funcionalidade. A topologia obtida e suas análises pelo MEF estão apresentadas nas figuras 6.46 e 6.47. As características desta topologia são mostradas na tabela 6.11 .

Como no caso sem restrições de volume, a topologia obtida também apresenta um mecanismo cujos deslocamentos da estrutura são muito parecidos para ambos os casos de carga e a implementação de restrições aplicadas aos índices de

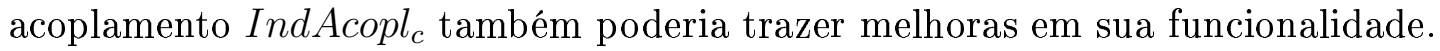
Apesar de não haver uma melhora na funcionalidade (maiores índices de acoplamento para ambos os casos de carga), pode-se verificar que a distribuição de 


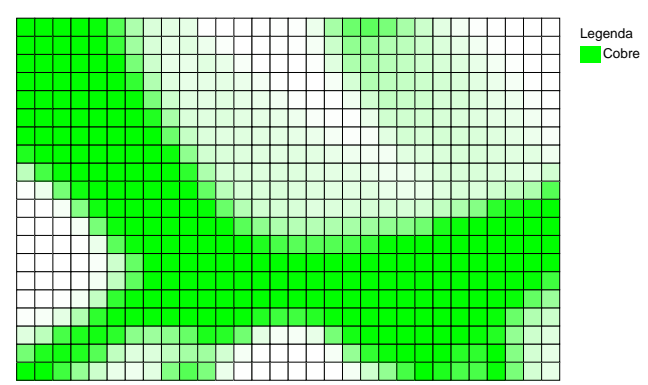

(a) 75 iterações

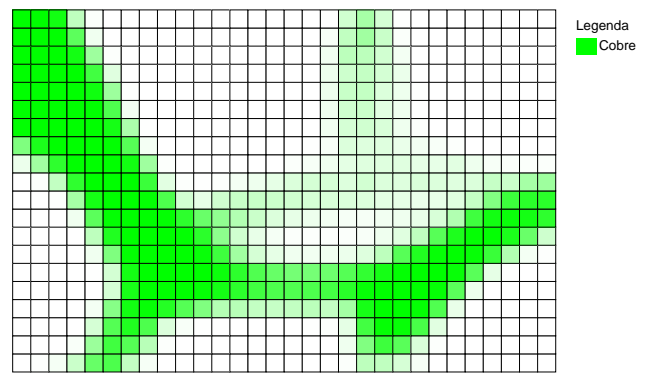

(c) 225 iterações

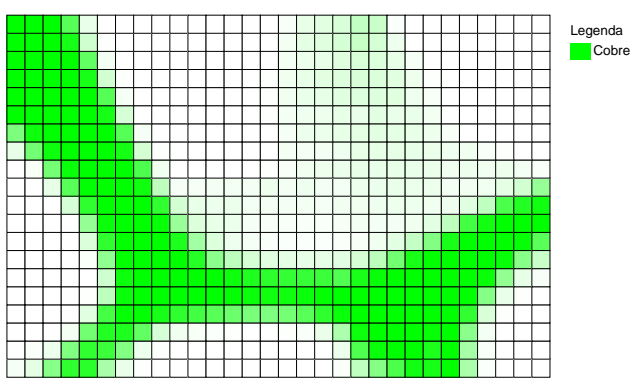

(b) 150 iterações

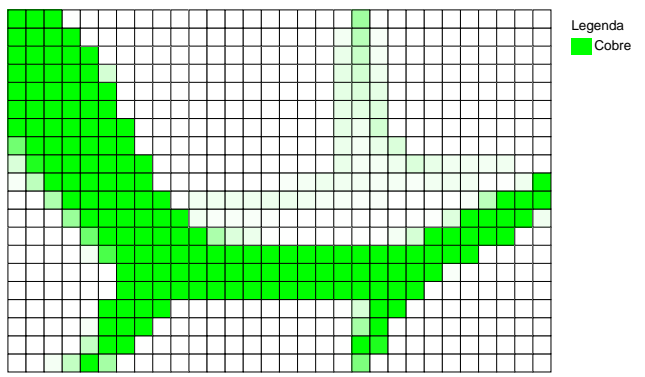

(d) 300 iterações

Figura 6.44: Atuador multiflexível (em cobre, com restrições de volume) Topologias intermediárias (vermelho - Ni; verde - Cu).

Tabela 6.11: Atuador multiflexível (em cobre, com restrições de volume) Características da topologia interpretada.

\begin{tabular}{|l|c|}
\hline Característica & Valor \\
\hline \hline Caso de Carga 1 & \\
- Deslocamento de Saída & $1,797 \mu \mathrm{m}$ \\
- Índice de Acoplamento & 4,215 \\
& \\
Caso de Carga 2 & \\
- Deslocamento de Saída & $1,033 \mu \mathrm{m}$ \\
- Índice de Acoplamento & $2,744 \times 10^{-1}$ \\
& \\
Volumes Relativos & $19,59 \%$ \\
- Cobre & $\mathbf{1 9 , 5 9 \%}$ \\
- Total &
\end{tabular}

temperatura neste caso (figuras 6.47(e) e 6.47(f)) se diferenciou mais entre os casos de carga que no caso sem restrição de volume (figuras 6.43(e) e 6.43(f)). Como no caso anterior, a menor flexibilidade no projeto por falta de um segundo material, além do vazio, pode ser percebida se compararmos com os resultados da seção 6.3.1, principalmente os que consideraram restrições de volume dos materiais. 


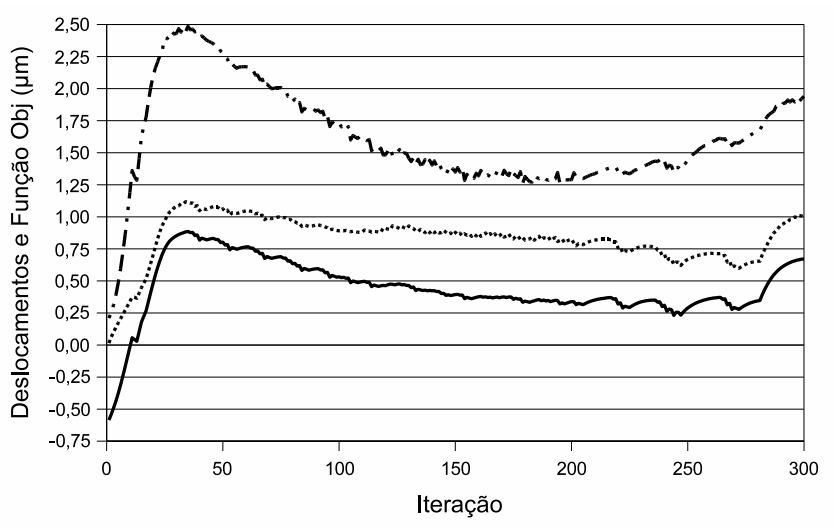

$\because$ Saida 1

$\because$ Func Obj

(a) Deslocamentos de saída e Função objetivo

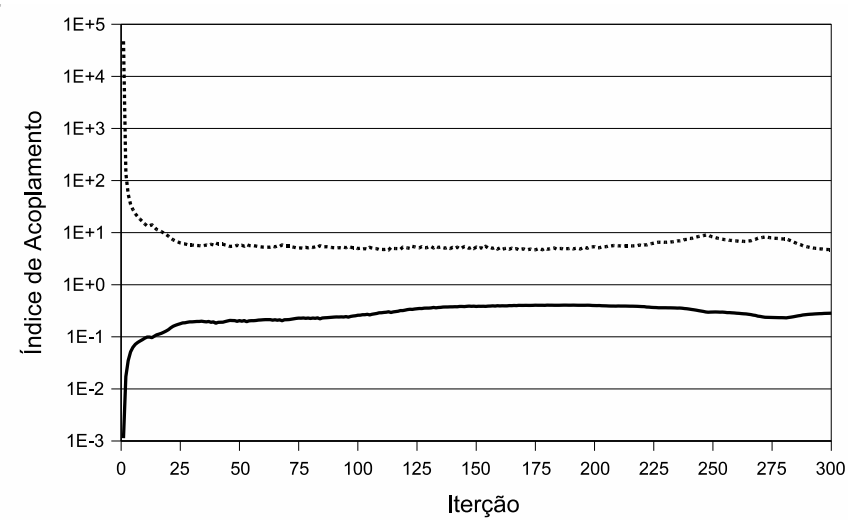

$\because$ Saida 1

(b) Acoplamentos dos deslocamentos de saída

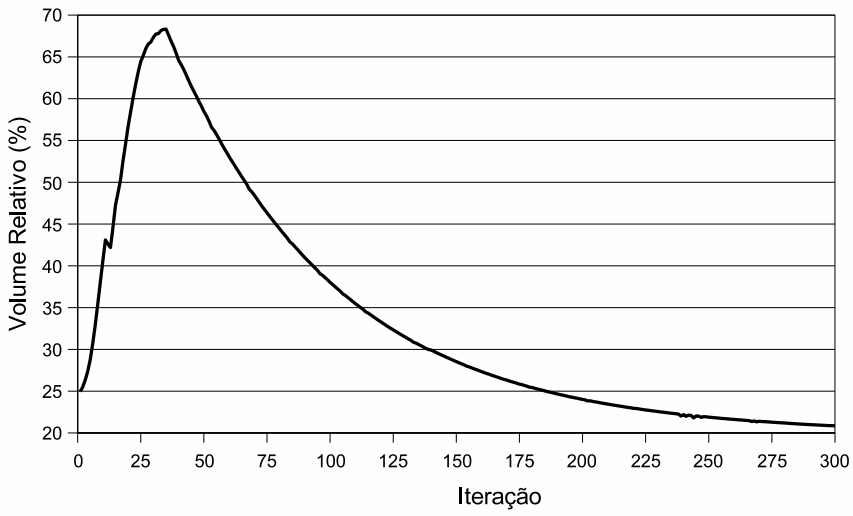

$\backslash$ cobre

(c) Volumes relativos dos materiais

Figura 6.45: Atuador multiflexível (em cobre, com restrições de volume) Curvas da otimização. 


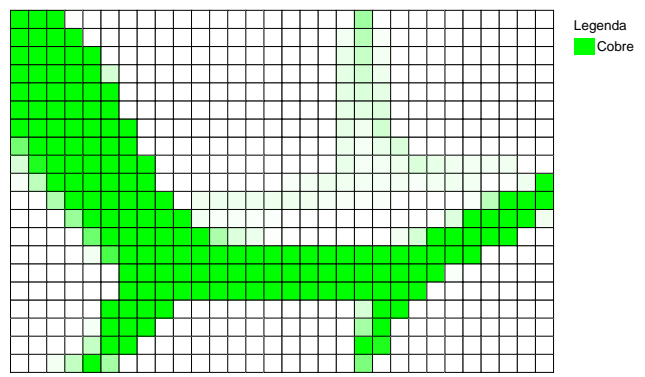

(a) Topologia resultante
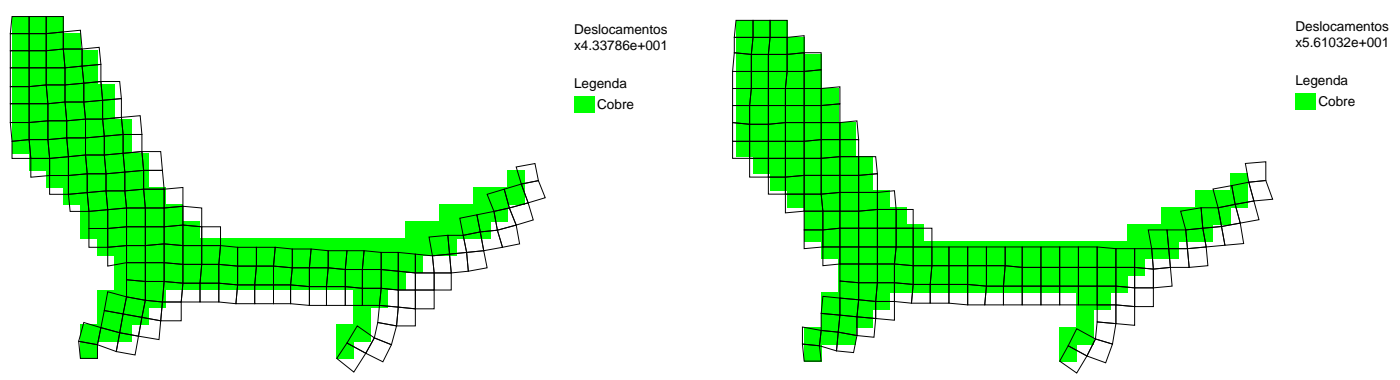

(b) Topologia interpretada e Deslocamentos (c) Topologia interpretada e Deslocamentos (Caso de Carga 1) (Caso de Carga 2)
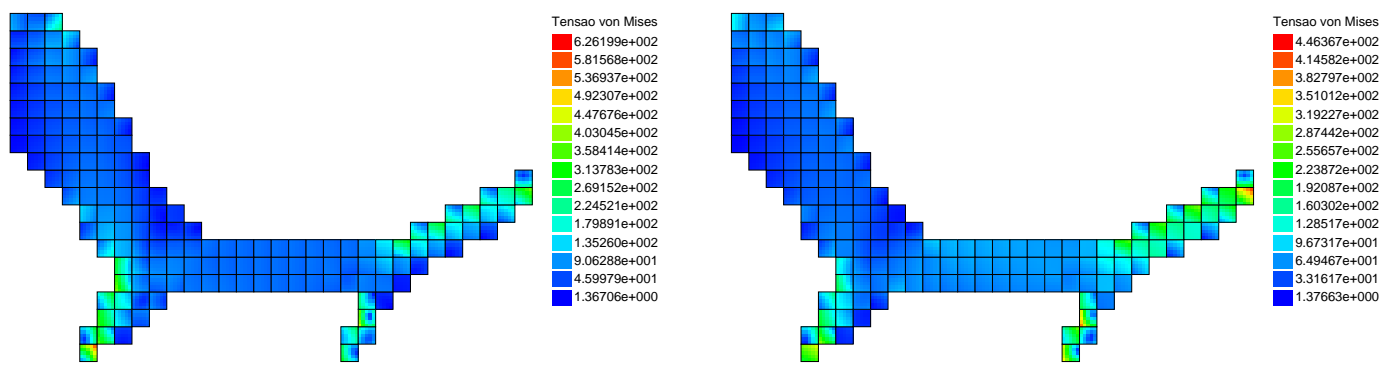

(d) Tensões de von Mises (Caso de Carga 1)

(e) Tensões de von Mises (Caso de Carga 2)

Figura 6.46: Atuador multiflexível (em cobre, com restrições de volume) Topologia final e interpretada, Análise termoelástica. 


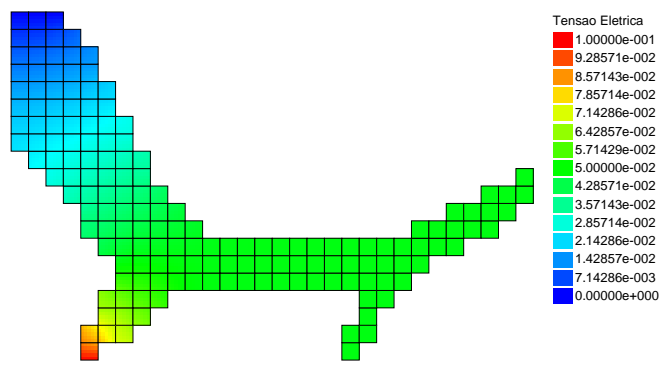

(a) Tensões Elétricas (Caso de Carga 1)

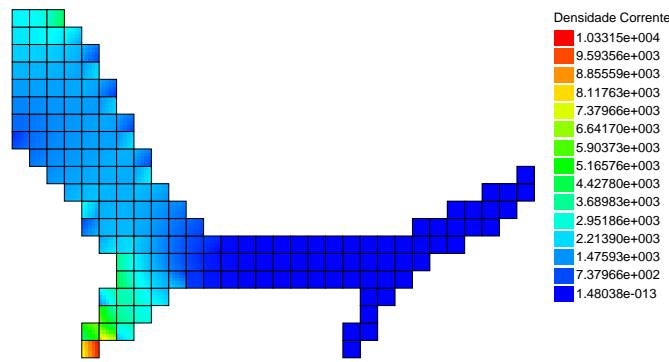

(c) Densidades de Corrente (Caso de Carga 1)
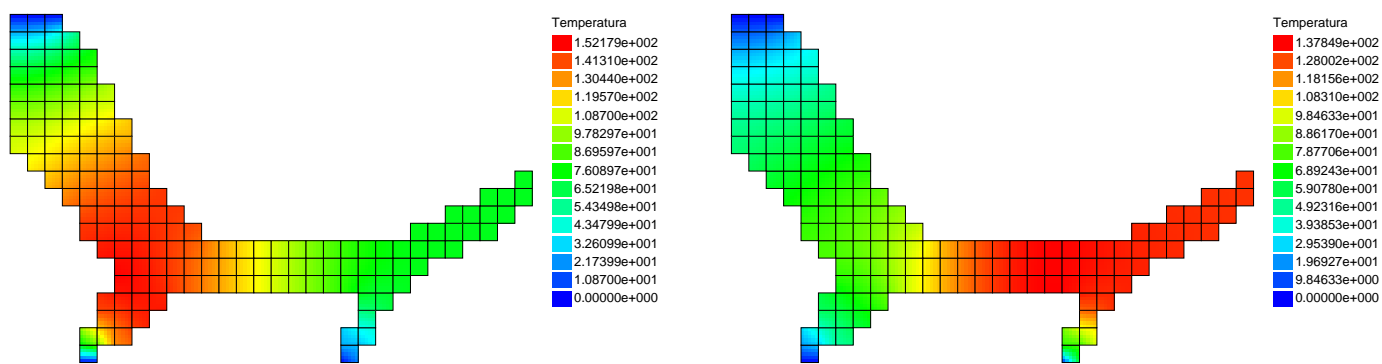

(e) Variações de Temperatura (Caso de Carga (f) Variações de Temperatura (Caso de Carga 1)

2)

Figura 6.47: Atuador multiflexível (em cobre, com restrições de volume) Análise elétrica e eletrotérmica. 


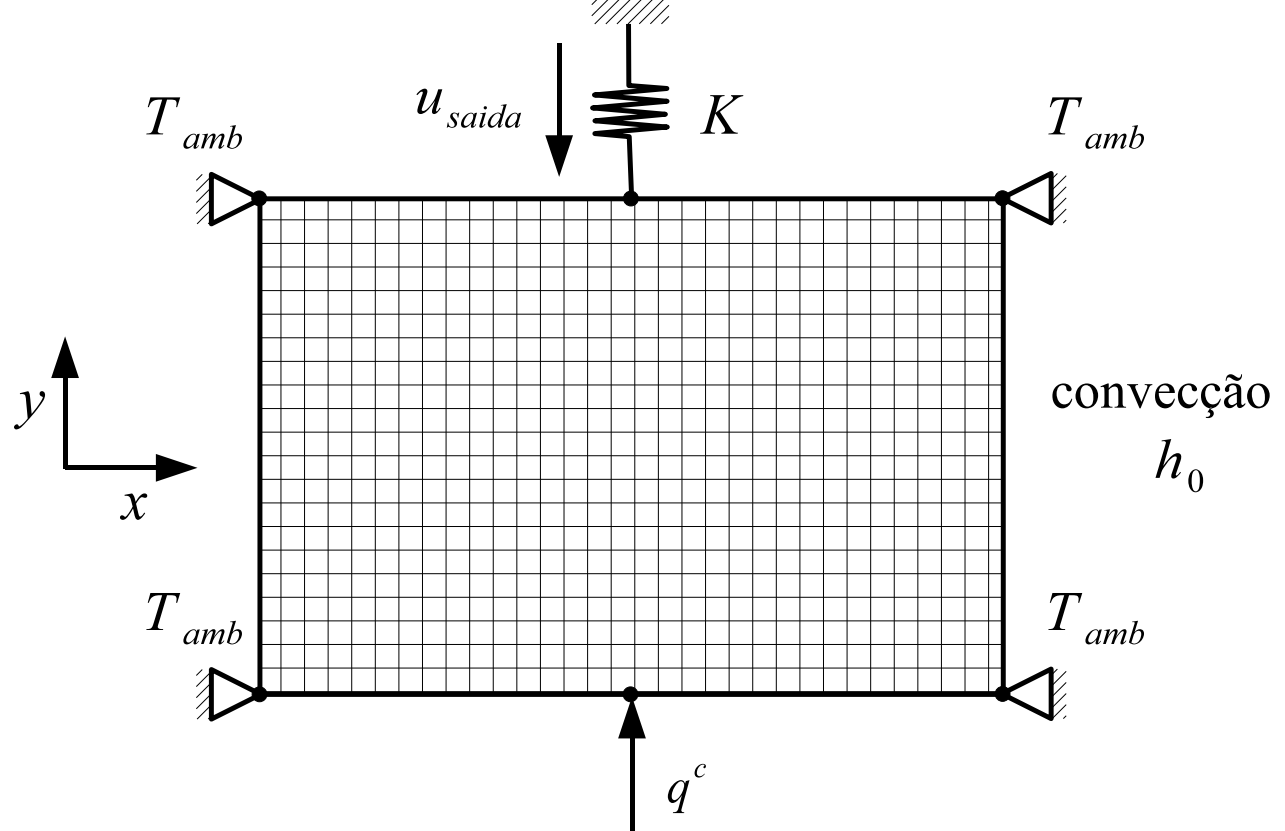

Figura 6.48: Domínio fixo estendido para o atuador de movimento inverso atuado por calor.

\subsection{Atuador de movimento inverso atuado por calor}

Atuação por fluxo de calor é uma alternativa à atuação eletrotermomecânica. Em termos de aplicação prática, esses micromecanismos poderiam ser atuados através de um fluxo de calor fornecido por um feixe de "laser", facilmente direcionado para o ponto desejado, ao invés da aplicação de tensão elétrica, como é feito no caso da atuação eletrotermomecânica.

Utilizando a idéia do atuador de movimento inverso, a atuação por tensão elétrica foi substituída por um fluxo de calor nodal concentrado de $q^{c}=0,1 \mathrm{~W}$ introduzido num ponto intermediário do lado inferior do domínio. Assim, o domínio tem dimensões $3 \mathrm{~mm} \times 2 \mathrm{~mm}$, discretizado em $30 \times 20$ elementos, e espessura de $15 \times 10^{-3} \mathrm{~mm}$. Nas quatro extremidades, foram aplicadas restrições em $x$ e $y$ e temperatura ambiente $T_{a m b}$. Sobre toda a superfície superior, foi considerado um coeficiente de conveç̧ão $h_{0}=2,5 \times 10^{-4} \mathrm{~W} / \mathrm{mm}^{2} \cdot \mathrm{K}$. Deseja-se maximizar o deslocamento $u_{\text {saida }}$, onde uma peça de rigidez $K=200 \mathrm{~N} / \mathrm{mm}$ é atuada. A figura 6.48 ilustra o domínio fixo estendido e as condições de contorno aplicadas. Uma simetria entre as metades esquerda e direita foi imposta para estes resultados. 


\subsubsection{Uso de dois materiais, além do vazio}

Como na seção 6.1, foram usados os mesmos dois materiais: silício e dióxido de silício, cujas propriedades estão relacionadas na tabela 6.1. O mesmo modelo de interpolação de material foi utilizado, variando os desvios padrão $\sigma_{m}$ de 0,3 a 0,1 de acordo com coeficientes de continuação $\omega_{D P_{m}}=0,01$ (com $m$ denotando o material referido).

Na primeira iteração do processo da otimização, todas as pseudo-densidades foram consideradas uniformes e com valor igual a 1,5, valor escolhido por estar entre as posições dos dois picos $\left(\mu_{m}\right.$, para $m=1$ e 2$)$.

O filtro espacial foi utilizado com raio igual a 0,11 , de modo a abranger apenas os elementos imediatamente na sua vizinhança na discretização considerada.

\section{Sem Restrições de Volume dos Materiais}

Inicialmente, sem considerar as restrições de volume de cada material, uma topologia ótima foi obtida após 200 iterações, que consumiram 11 minutos de processamento. O filtro espacial foi desativado a partir da iteração 150 do processo para que a quantidade de materiais intermediários fosse reduzida. A figura 6.49 mostra as topologias intermediárias encontradas até a solução final e a figura 6.50, as curvas do deslocamento de saída e dos volumes relativos dos materiais.

Sobre a topologia resultante, foi feita uma interpretação de modo a tornála factível: os elementos com mais de $30 \%$ do material predominante tiveram a pseudo-densidade alterada para $100 \%$ desse mesmo material; caso contrário, recebe o valor do vazio para a pseudo-densidade. A topologia obtida e suas análises pelo MEF estão apresentadas na figura 6.51. As características desta topologia são mostradas na tabela 6.12 .

Tabela 6.12: Atuador de movimento inverso atuado por calor - Características da topologia final.

\begin{tabular}{|l|c|}
\hline Característica & Valor \\
\hline \hline Deslocamento de Saída & $6,396 \times 10^{-1} \mu \mathrm{m}$ \\
Volumes Relativos & \\
- Silício & $21,00 \%$ \\
- Dióxido de silício & $30,33 \%$ \\
- Total & $\mathbf{5 1 , 3 3 \%}$ \\
\hline
\end{tabular}

Como no caso do atuador de movimento inverso atuado por tensão elétrica, 


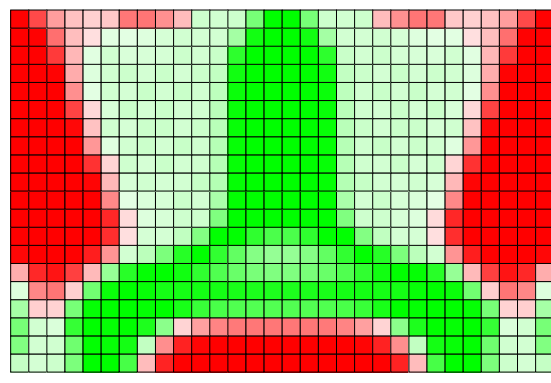

(a) 50 iterações

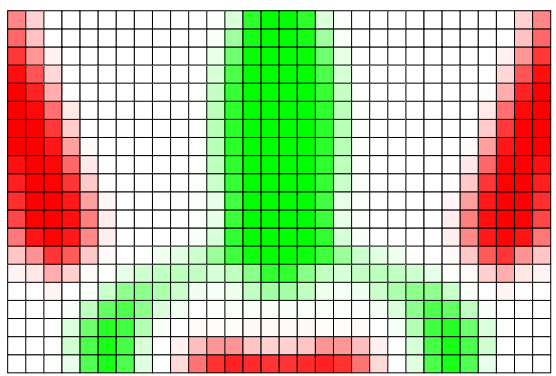

(c) 150 iterações

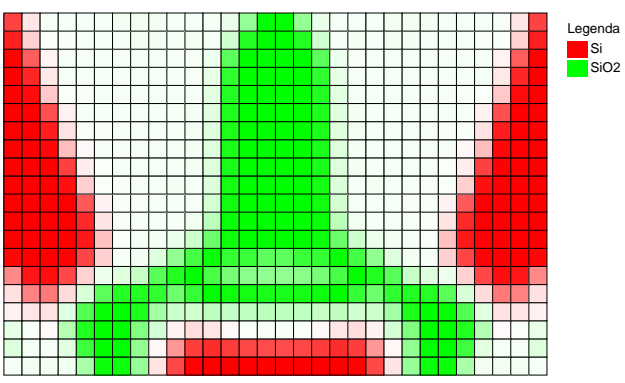

(b) 100 iterações

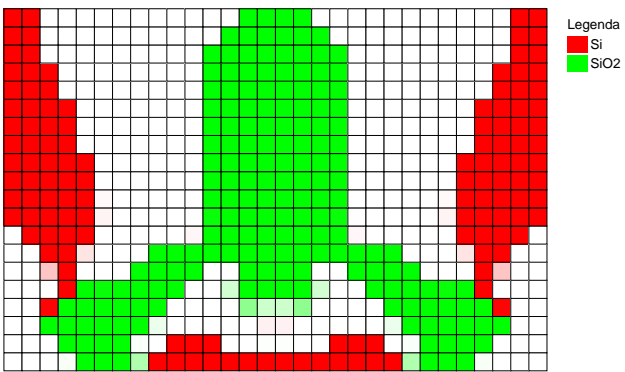

(d) 200 iterações

Figura 6.49: Atuador de movimento inverso atuado por calor - Topologias intermediárias (vermelho - $\mathrm{Si}$; verde - $\mathrm{SiO}_{2}$ ).

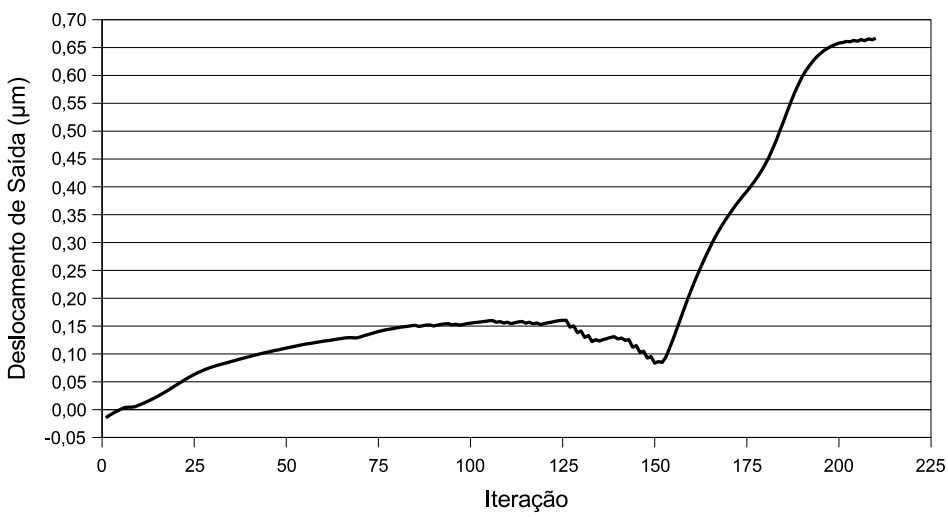

(a) Deslocamento de saída

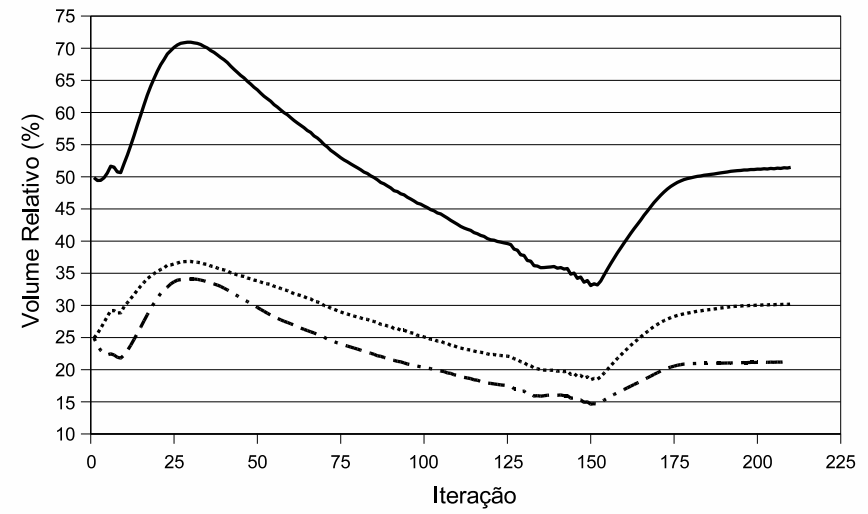

\begin{tabular}{|l|}
\hline \\
$\because \mathrm{Si}$ \\
$\because \cup$ SiO2 \\
$\searrow$ Total \\
\hline
\end{tabular}

(b) Volumes relativos dos materiais

Figura 6.50: Atuador de movimento inverso atuado por calor - Curvas da otimização. 


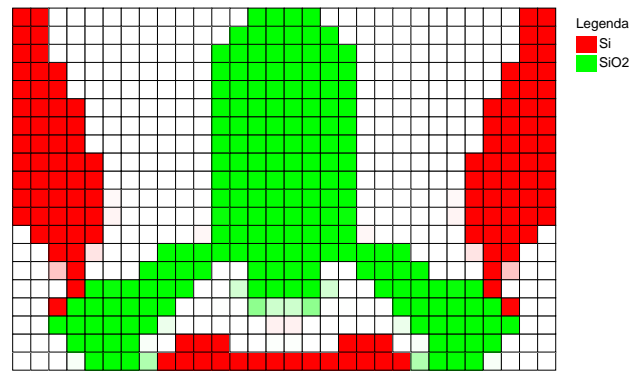

(a) Topologia resultante (vermelho - $\mathrm{Si}$; verde $-\mathrm{SiO}_{2}$ )

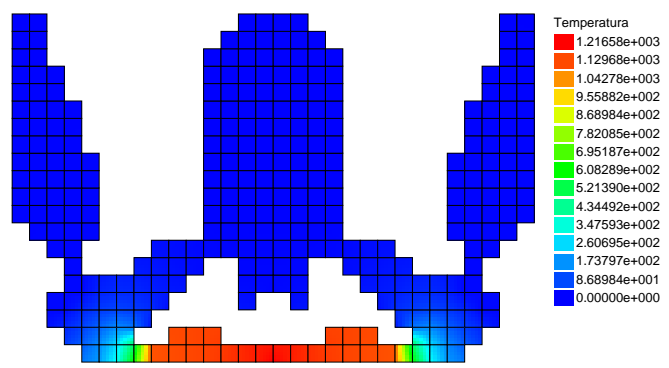

(c) Variações de Temperatura

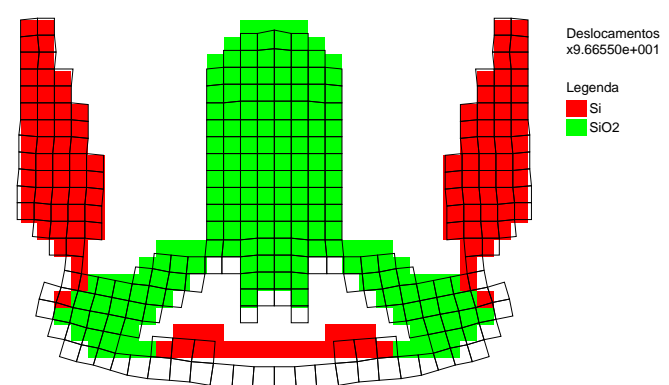

(b) Topologia interpretada e Deslocamentos

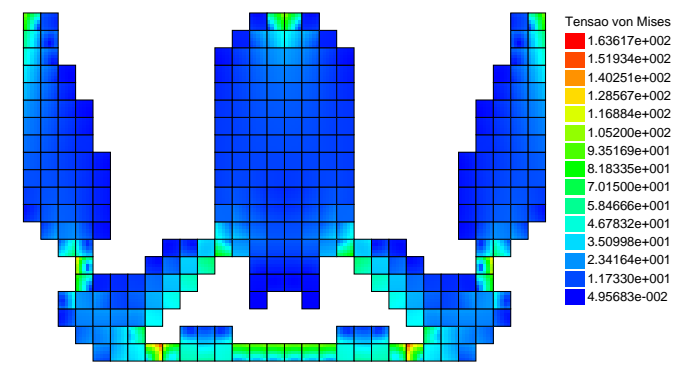

(d) Tensões de von Mises

Figura 6.51: Atuador de movimento inverso atuado por calor - Topologia final e Análises.

a haste que atua o grau de liberdade de saída é composta de dióxido de silício, um isolante térmico, para evitar seu aquecimento e conseqüente expansão que reduziria o desempenho do mecanismo. Esse material também foi usado para isolar a parte em silício que recebe o fluxo de calor dos pontos da estrutura em que a temperatura ambiente é imposta. Desse modo, menos calor é perdido e um maior deslocamento é conseguido. Note que existe um prolongamento inferior na haste em dióxido de silício que parece não contribuir na funcionalidade do mecanismo. A consideração de restrições de volume pode ajudar a reduzir o uso de material em pontos desnecessários.

\section{Com Restrições de Volume dos Materiais}

O próximo resultado foi obtido ao impor restrições de volume de para o silício e dióxido de silício, de $20 \%$ do volume total para cada um. Iniciou-se a otimização com volumes máximos de $100 \%$ de volume relativo para cada material, reduzindoos de acordo com um coeficiente de continuação $\omega_{R V_{m}}$ de 0,02 . Obteve-se a topologia ótima após 200 iterações da rotina de otimização. Para reduzir densidades intermediárias, o filtro espacial foi desativado a partir da iteração 150. A figura 6.52 mostra as topologias intermediárias encontradas até a solução final e a figura 6.53 , as curvas do deslocamento de saída e dos volumes relativos 


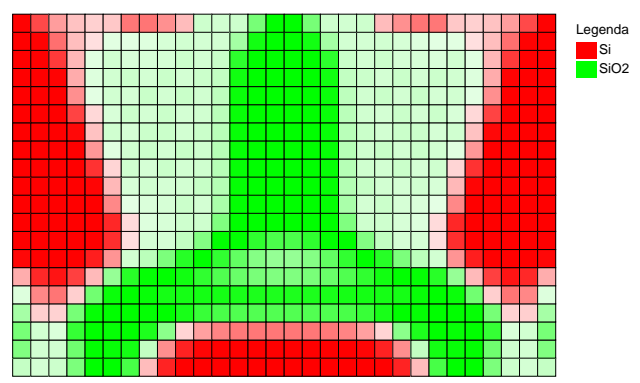

(a) 50 iterações

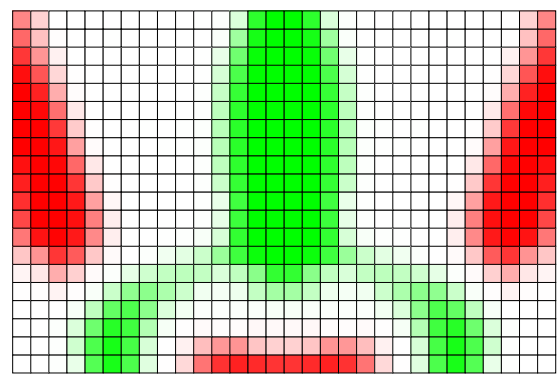

(c) 150 iterações

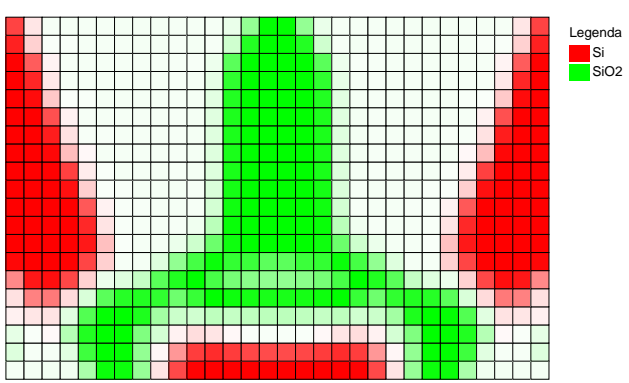

(b) 100 iterações

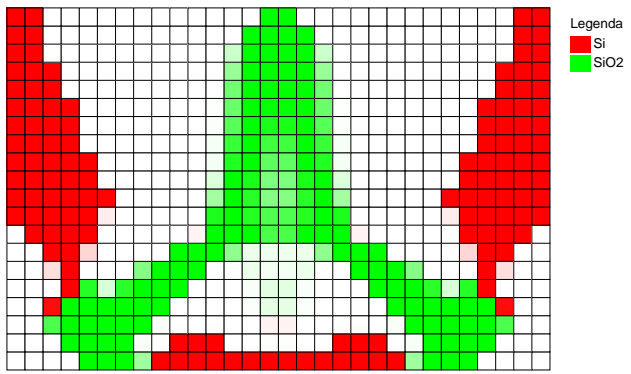

(d) 200 iterações

Figura 6.52: Atuador de movimento inverso atuado por calor (com restrições de volume) - Topologias intermediárias (vermelho - $\mathrm{Si}$; verde - $\mathrm{SiO}_{2}$ ) .

dos materiais.

Sobre a topologia resultante, foi feita a mesma interpretação do caso anterior: os elementos com mais de $30 \%$ do material predominante tiveram a pseudodensidade alterada para $100 \%$ desse mesmo material; caso contrário, recebe o valor do vazio para a pseudo-densidade. A topologia obtida e suas análises pelo MEF estão apresentadas na figura 6.54. As características desta topologia são mostradas na tabela 6.13 .

Tabela 6.13: Atuador de movimento inverso atuado por calor (com restrições de volume) - Características da topologia final.

\begin{tabular}{|l|c|}
\hline Característica & Valor \\
\hline \hline Deslocamento de Saída & $6,279 \times 10^{-1} \mu \mathrm{m}$ \\
Volumes Relativos & \\
- Silício & $21,33 \%$ \\
- Dióxido de silício & $23,67 \%$ \\
- Total & $\mathbf{4 5 , 0 0 \%}$ \\
\hline
\end{tabular}

A topologia obtida possui mesmas funcionalidades e características do resultado em que não foram consideradas restrições de volume dos materiais. As restrições de volume dos materiais tiveram efeito principalmente sobre a distribuição do dióxido de silício, já que a quantidade de silício não se alterou de forma significativa. Conforme esperado, a restrição de volume para o dióxido 


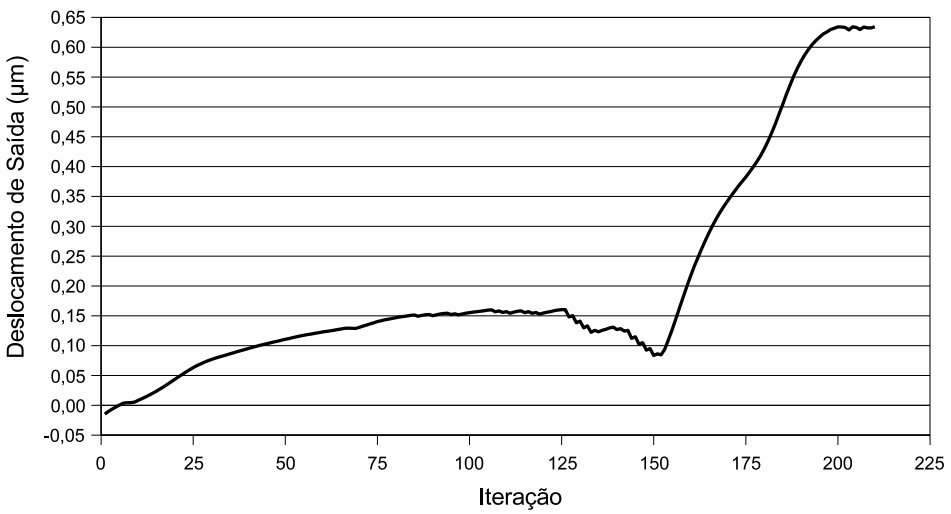

(a) Deslocamento de saída

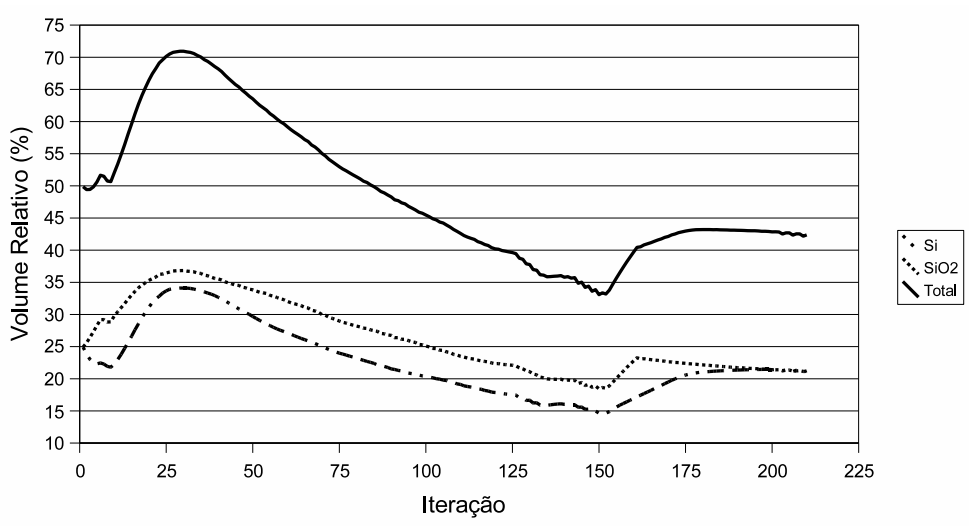

(b) Volumes relativos dos materiais

Figura 6.53: Atuador de movimento inverso atuado por calor (com restrições de volume) - Curvas da otimização.

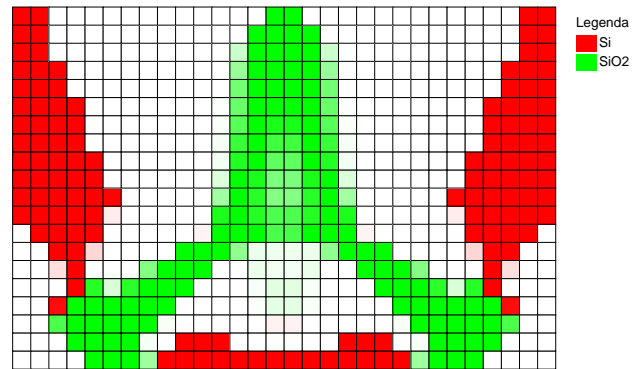

(a) Topologia resultante (vermelho - $\mathrm{Si}$; verde $\left.-\mathrm{SiO}_{2}\right)$

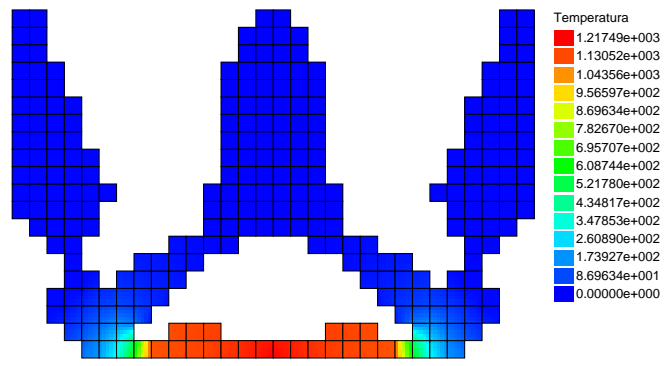

(c) Variações de Temperatura

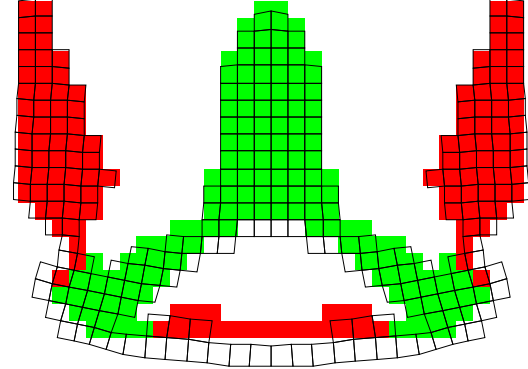

Deslocamentos
$\times 1.00261 \mathrm{e}+002$

Legenda

(b) Topologia interpretada e Deslocamentos
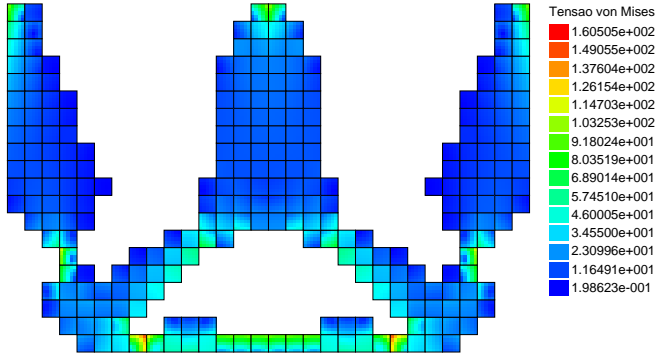

(d) Tensões de von Mises

Figura 6.54: Atuador de movimento inverso atuado por calor (com restrições de volume) - Topologia final e Análises. 
de silício eliminou as partes sem utilidade do mecanismo. A redução do volume desse material não teve maior impacto no desempenho, reduzindo o deslocamento de saída em menos de $2 \%$.

\subsubsection{Uso de três materiais, além do vazio}

O mesmo tipo de atuador, com mesmo domínio fixo estendido e condições de contorno, também foi projetado com a consideração de um material adicional, o níquel, cujas propriedades foram apresentadas na tabela 6.4.

Em relação ao modelo de interpolação de material utilizado, foi acrescentado um termo com pico para a pseudo-densidade 3,0, representando o Material 3 (Níquel). Os desvios padrão $\sigma_{m}$ se iniciaram em 0,5, caindo para 0,15 de acordo com coeficientes de continuação $\omega_{D P_{m}}=0,008$ (com $m$ denotando o material referido).

Na primeira iteração do processo da otimização, todas as pseudo-densidades foram consideradas uniformes e com valor igual a 2,0, valor escolhido por ser a média das posições dos três picos $\left(\mu_{m}\right.$, para $m=1,2$ e 3$)$.

\section{Sem Restrições de Volume dos Materiais}

Sem considerar restrições de volume para os materiais, uma topologia ótima foi obtida após 340 iterações, que consumiram 17 minutos de processamento. $\mathrm{O}$ filtro espacial foi desativado a partir da iteração 150 do processo para que a quantidade de materiais intermediários fosse reduzida. A figura 6.55 mostra as topologias intermediárias encontradas até a solução final e a figura 6.56, as curvas do deslocamento de saída e dos volumes relativos dos materiais.

Sobre a topologia resultante, foi feita uma interpretação de modo a tornála factível: os elementos com mais de $30 \%$ do material predominante tiveram a pseudo-densidade alterada para 100\% desse mesmo material; caso contrário, recebe o valor do vazio para a pseudo-densidade. Alguns elementos entre a parte inferior em Ni e a parte em $\mathrm{SiO}_{2}$ foram definidos como $\mathrm{SiO}_{2}$ para que a parte em Ni não ficasse desconexa e outros foram eliminados por não terem sentido físico. A topologia obtida e suas análises pelo MEF estão apresentadas na figura 6.57. As características desta topologia são mostradas na tabela 6.14.

A topologia obtida mantém a mesma funcionalidade observada nos resultados da seção 6.4.1. Como no caso anterior, a não consideração de restrições de volume de material permitiu o emprego de material em pontos desnecessários. 


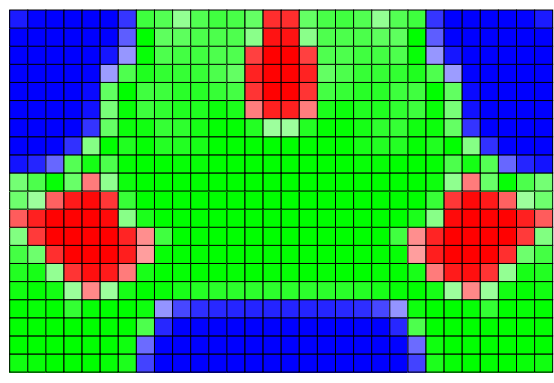

(a) 85 iterações

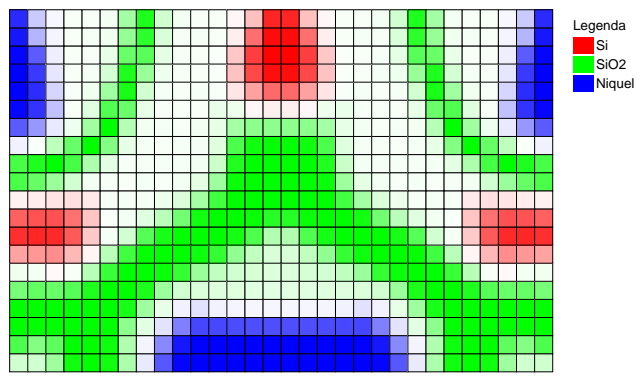

(c) 255 iterações

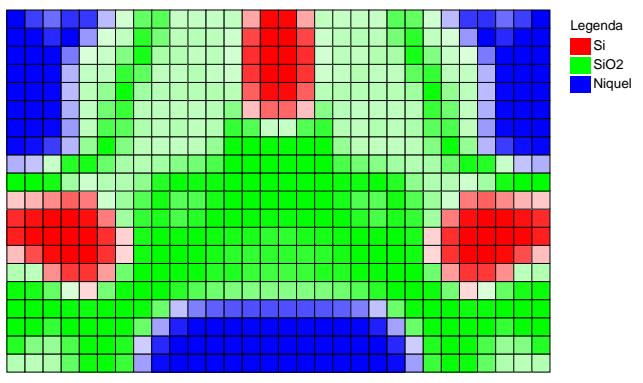

(b) 170 iterações

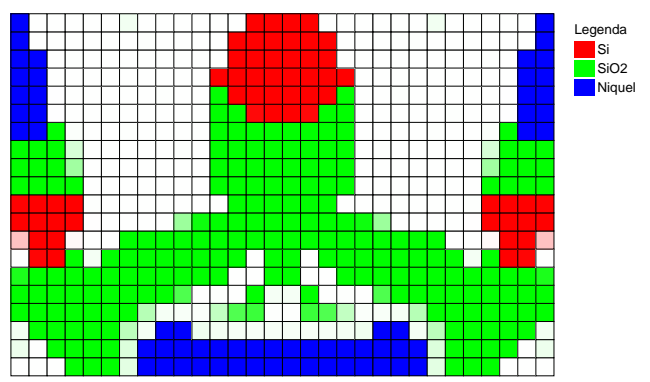

(d) 340 iterações

Figura 6.55: Atuador de movimento inverso atuado por calor (3 materiais) Topologias intermediárias (vermelho - $\mathrm{Si}$; verde - $\mathrm{SiO}_{2}$; azul - Ni).

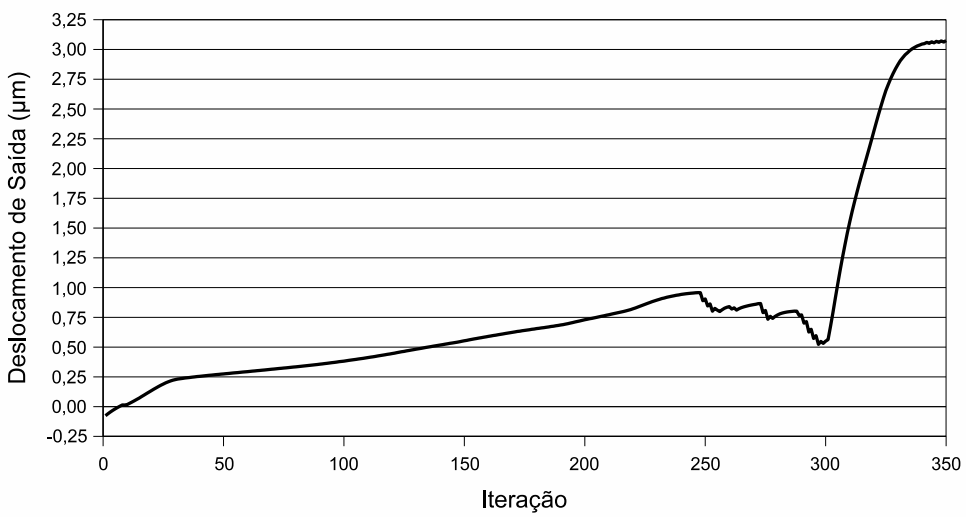

(a) Deslocamento de saída

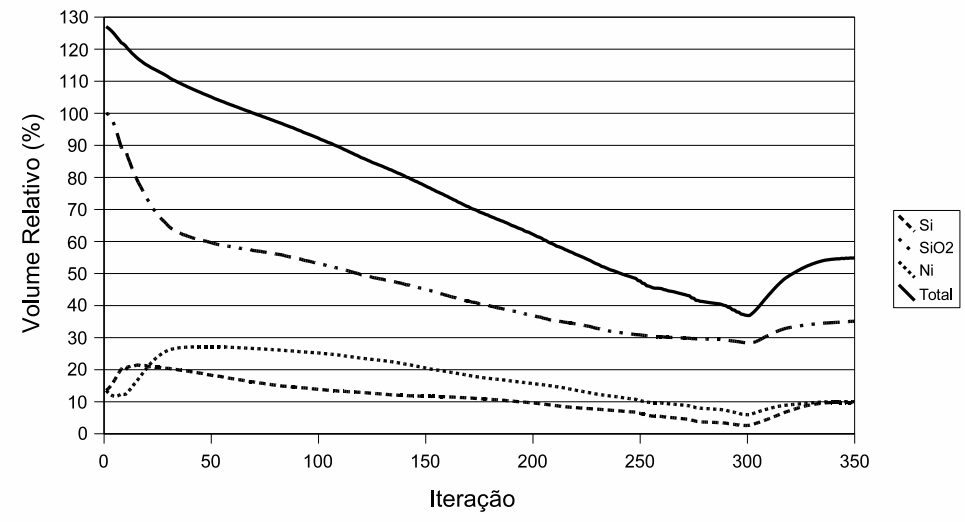

(b) Volumes relativos dos materiais

Figura 6.56: Atuador de movimento inverso atuado por calor (3 materiais) Curvas da otimização. 


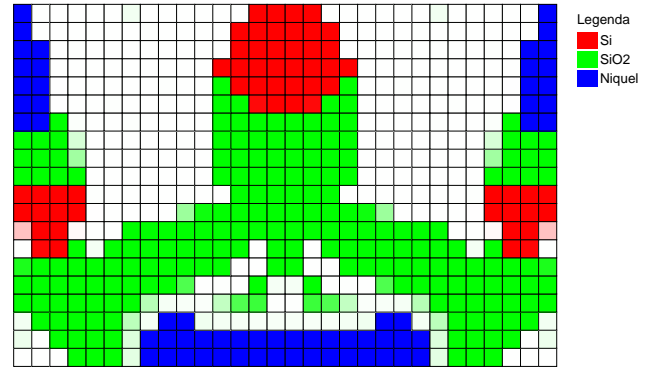

(a) Topologia resultante (vermelho - $\mathrm{Si}$; verde $-\mathrm{SiO}_{2} ;$ azul - Ni)

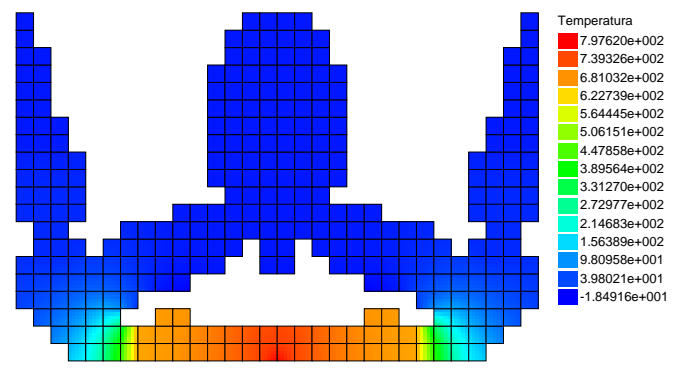

(c) Variações de Temperatura

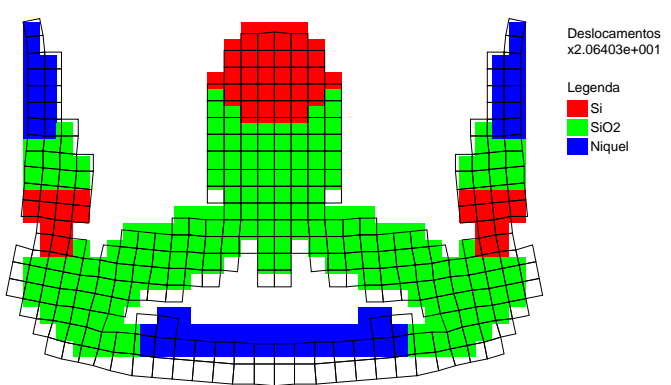

(b) Topologia interpretada e Deslocamentos

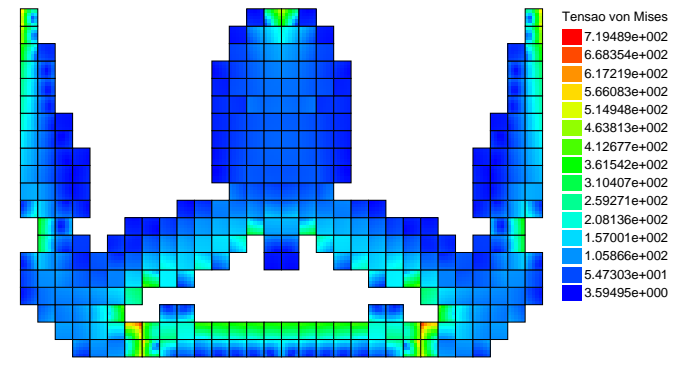

(d) Tensões de von Mises

Figura 6.57: Atuador de movimento inverso atuado por calor (3 materiais) Topologia final e Análises.

Tabela 6.14: Atuador de movimento inverso atuado por calor (3 materiais) Características da topologia final.

\begin{tabular}{|l|c|}
\hline Característica & Valor \\
\hline \hline Deslocamento de Saída & $3,050 \mu \mathrm{m}$ \\
Volumes Relativos & \\
- Silício & $9,67 \%$ \\
- Dióxido de silício & $34,33 \%$ \\
- Níquel & $10,00 \%$ \\
- Total & $\mathbf{5 4 , 0 0 \%}$ \\
\hline
\end{tabular}

O níquel, de maior coeficiente de expansão térmica, foi empregado de forma a receber o fluxo de calor, substituindo o silício como material responsável pela atuação termomecânica. O silício, com módulo de Young similar ao níquel, teve participação mais estrutural no projeto. Por ser mais rígido que o dióxido de silício, substituiu esse material na atuação do grau de liberdade de saída de modo a permitir uma menor deformação do mecanismo durante o esforço.

\section{Com Restrições de Volume dos Materiais}

Impondo restrições de volume de $20 \%$ do volume total para cada um dos materiais, iniciou-se a otimização com volumes máximos de $100 \%$ de volume relativo, reduzindo-os de acordo com um coeficiente de continuação $\omega_{R V_{m}}$ de 0,015 . A 


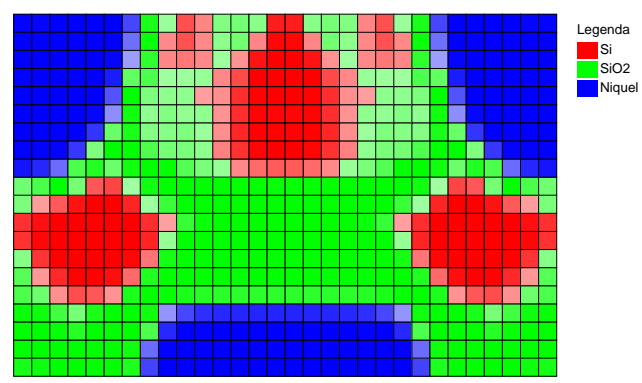

(a) 85 iterações

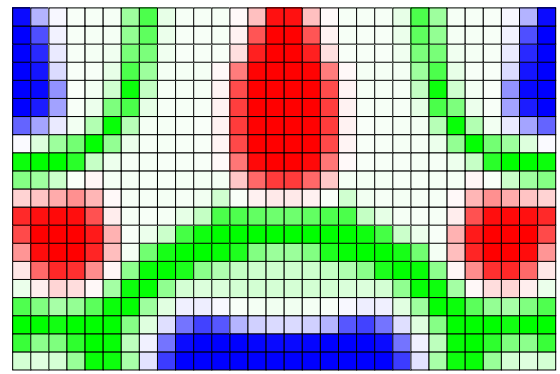

(c) 255 iterações

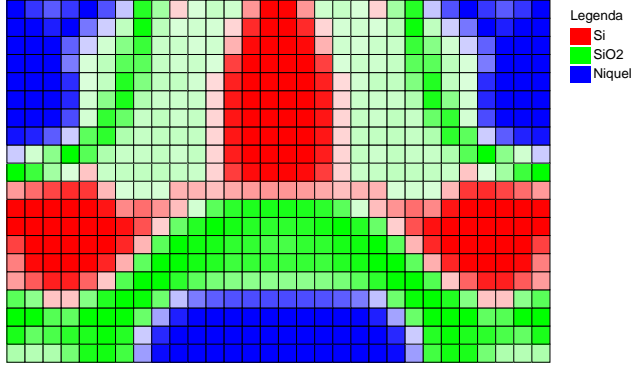

(b) 170 iterações

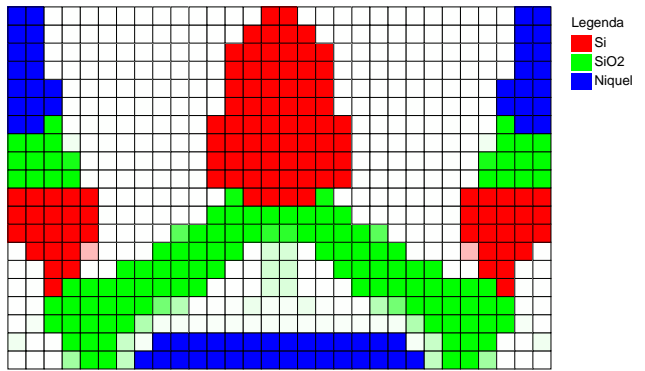

(d) 350 iterações

Figura 6.58: Atuador de movimento inverso atuado por calor (3 materiais, com restrições de volume) - Topologias intermediárias (vermelho - Si; verde - $\mathrm{SiO}_{2}$ ).

topologia ótima foi obtida após 350 iterações da rotina de otimização. Para reduzir densidades intermediárias, o filtro espacial foi desativado a partir da iteração 150. A figura 6.58 mostra as topologias intermediárias encontradas até a solução final e a figura 6.59 , as curvas do deslocamento de saída e dos volumes relativos dos materiais.

Sobre a topologia resultante, foi feita uma interpretação de modo a tornála factível: os elementos com mais de $50 \%$ do material predominante tiveram a pseudo-densidade alterada para 100\% desse mesmo material; caso contrário, recebe o valor do vazio para a pseudo-densidade. Alguns elementos entre a parte inferior em $\mathrm{Ni}$ e a parte em $\mathrm{SiO}_{2}$ foram definidos como $\mathrm{SiO}_{2}$ para que a parte em Ni não ficasse desconexa. A topologia obtida e suas análises pelo MEF estão apresentadas na figura 6.60. As características desta topologia são mostradas na tabela 6.15 .

A topologia obtida possui mesma funcionalidade do caso anterior, mas com algumas alterações significativas devido à consideração das restrições de volume dos materiais. Ao restringir a quantidade de dióxido de silício, a otimização eliminou as partes sem utilidade do mecanismo e substituiu esse material pelo silício. A quantidade de níquel não se alterou significativamente. Essas mudanças na topologia resultaram em uma topologia ligeiramente diferente, mas que permitiu um aumento de mais de $20 \%$ no deslocamento de saída. 


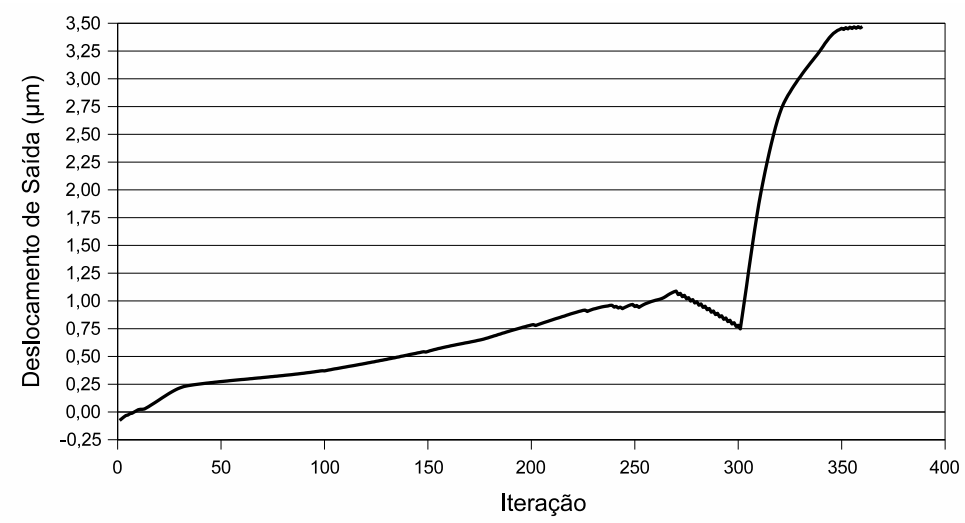

(a) Deslocamento de saída

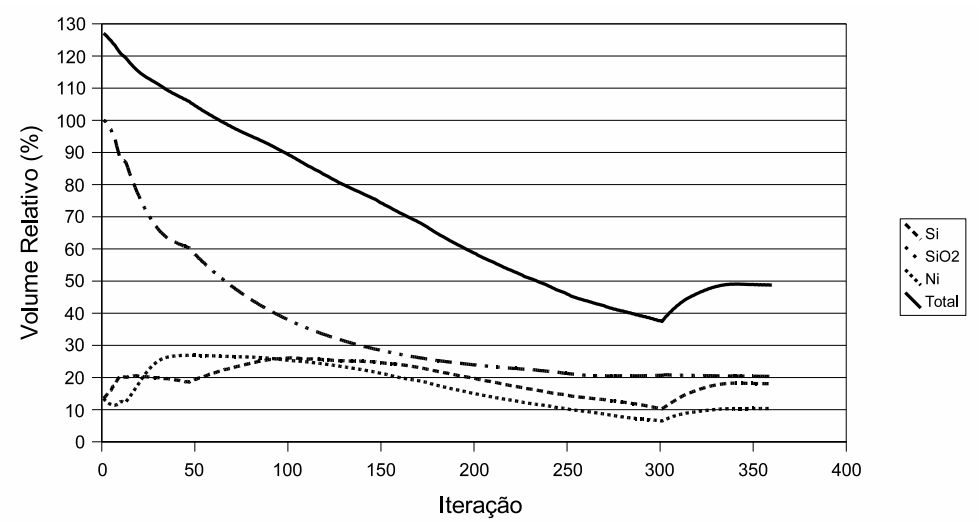

(b) Volumes relativos dos materiais

Figura 6.59: Atuador de movimento inverso atuado por calor (3 materiais, com restrições de volume) - Curvas da otimização.

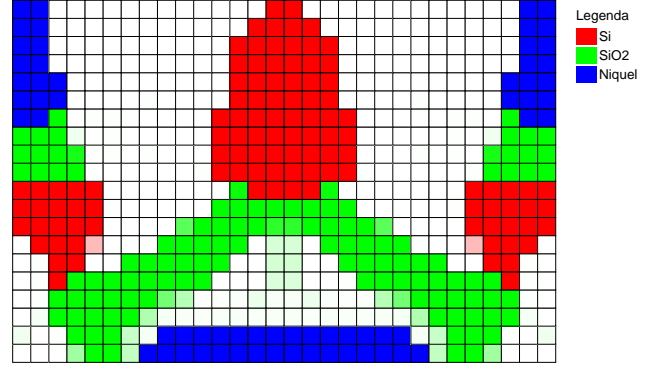

(a) Topologia resultante (vermelho - $\mathrm{Si}$; verde $-\mathrm{SiO}_{2} ;$ azul - Ni)

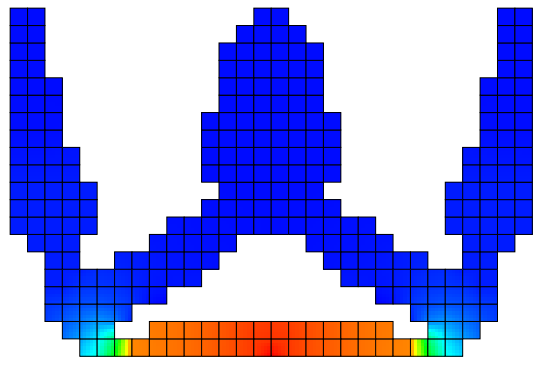

(c) Variações de Temperatura

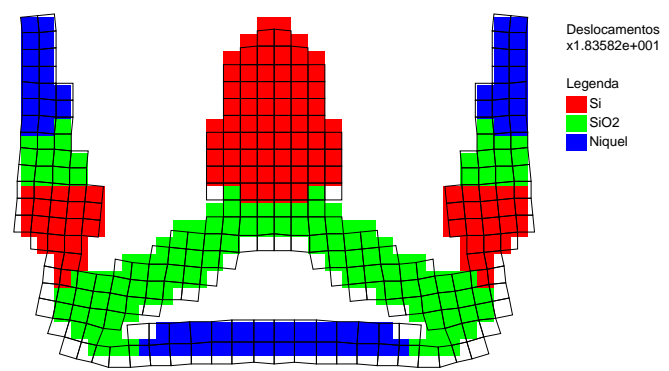

(b) Topologia interpretada e Deslocamentos

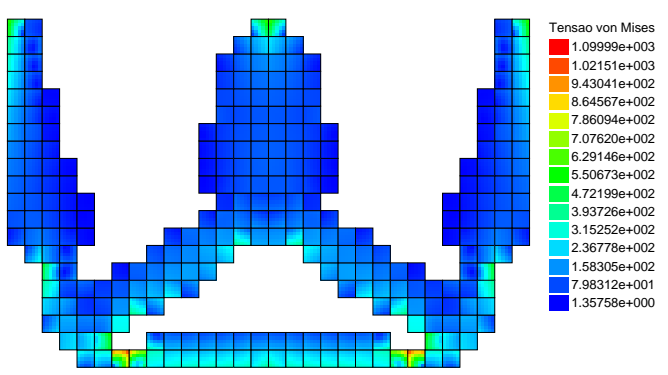

(d) Tensões de von Mises

Figura 6.60: Atuador de movimento inverso atuado por calor (3 materiais, com restrições de volume) - Topologia final e Análises. 
Tabela 6.15: Atuador de movimento inverso atuado por calor (3 materiais, com restrições de volume) - Características da topologia final.

\begin{tabular}{|l|c|}
\hline Característica & Valor \\
\hline \hline Deslocamento de Saída & $3,746 \mu \mathrm{m}$ \\
Volumes Relativos & \\
- Silício & $18,00 \%$ \\
- Dióxido de silício & $20,00 \%$ \\
- Níquel & $10,33 \%$ \\
- Total & $\mathbf{4 8 , 3 3 \%}$ \\
\hline
\end{tabular}




\section{Conclusões e Sugestão de Trabalhos Futuros}

O problema de otimização topológica de MEMS eletrotermomecânicos multifásicos foi resolvido utilizando a PLS, com o material sendo interpolado por um dos métodos das densidades (função de pico). Conhecendo-se as instabilidades numéricas normalmente ocorridas usando esse tipo de modelo de material, foram tomadas as medidas para contorná-las mencionadas no texto: para reduzir o problema da não-unicidade da solução, foi usado o método da continuação sobre os desvios padrão das funções de pico; esse mesmo método da continuação aplicado sobre os desvios padrão, por consistir numa penalização das densidades intermediárias, diminui a formação de escalas de cinza ("grayscale"). Um filtro espacial foi implementado como uma tentativa de se reduzir os problemas de instabilidade de tabuleiro ("checkerboard") e dependência de malha. Para verificar a implementação do MOT, um resultado de Yin e Ananthasuresh (2002) foi reproduzido sem a utilização de restrições de volume. Com as restrições de volume, a topologia ótima teve um desempenho melhor, mas a simetria foi perdida. O mesmo tipo de mecanismo foi obtido com a consideração de três materiais, além do vazio.

Um estudo da influência de alguns parâmetros da otimização foi feito, tais como a rigidez da peça atuada, restrições de volume, uso do filtro espacial, coeficiente de continuação e parâmetros da função de pico (média e desvios padrão). Foi constatado que a rigidez da peça atuada influi na integridade estrutural do mecanismo, tornando-o mais robusto quando valores maiores de rigidez são empregados. Valores muito baixos para a rigidez da peça atuada estão relacionados a baixa integridade estrutural do mecanismo, muitas vezes permitindo uma grande quantidade de elementos com escalas de cinza.

Em relação às restrições de volume, concluiu-se que podem influenciar no resultado final, mesmo que não estejam ativas no fim da otimização. Limitando os volumes dos materiais durante a rotina, pode-se estar alterando o máximo local para onde a topologia caminhava, resultando em outra diferente. Essa alteração 
do máximo local foi evitada variando-se continuamente os valores das restrições de volume, começando com limites máximos de volume altos e reduzindo-os a cada iteração, de modo semelhante ao que foi feito com os desvios padrão das funções de pico (método da continuação).

Com o uso do filtro espacial, que limita os gradientes de material dentro de um raio em torno de um dado elemento, pode-se controlar a dependência da malha, complexidade da estrutura e instabilidades de tabuleiro do resultado. Em relação à dependência da malha, o uso do filtro se mostrou eficaz, conseguindo um refinamento de uma topologia ao aumentar a discretização da malha empregada. O controle da complexidade pelo filtro espacial teve papel importante na reprodução do resultado da literatura, já que em Yin e Ananthasuresh (2002) a complexidade pode ter sido reduzida pela implementação da convecção lateral dos elementos aliada à maximização do deslocamento. No entanto, por causa do seu princípio de funcionamento, o filtro tende a favorecer a existência de materiais intermediários fictícios (as escalas de cinza) e a retardar a otimização. Por isso, desativa-se o filtro a um determinado número de iterações do final do processo. A sua desativação prematura, antes que a resposta se aproxime mais de uma topologia sem estruturas espúrias, por exemplo, pode resultar em um número maior de iterações para se alcançar um mesmo resultado. Se a otimização seguir por um número relativamente elevado de iterações sem o uso do filtro, a topologia pode adquirir características do resultado obtido sem a filtragem, como estruturas espúrias, topologia complexa e não factível ou instabilidades de tabuleiro.

O coeficiente de continuação, que regula o quanto os desvios padrão são reduzidos por iteração, deve ser bem determinado de modo a não resultar em um número muito alto ou baixo de iterações antes da convergência. Caso seja muito pouca a redução dos desvios padrão (coeficiente muito baixo), o número de iterações necessárias (e o custo computacional) aumenta demais, mas as estruturas espúrias têm uma atenuação maior. Por outro lado, uma grande redução dos desvios padrão por iteração resulta em um número menor de iterações até que a convergência seja alcançada, mas a rotina pode não ser capaz de eliminar as estruturas que não contribuem para uma melhora no desempenho.

Em relação aos parâmetros da função de pico, concluiu-se que não são os valores das médias dos picos que influenciam os resultados, mas sim a separação entre elas. Uma alteração na separação entre as médias dos picos pode não ter efeito na otimização caso o mesmo fator seja aplicado aos desvios padrão iniciais.

O problema de otimização topológica de MEMS eletrotermomecânicos 
multifásicos foi estendido para considerar multiflexibilidade. Foram obtidos mecanismos compostos por dois materiais, além do vazio, com dois casos de carga, cada um com um deslocamento de saída correspondente. A multiflexibilidade foi obtida, apesar de um forte acoplamento entre os deslocamentos de saída. Para efeito de comparação, mecanismos compostos de apenas um material, além do vazio, foram projetados. As topologias obtidas permitiram verificar que a perda da flexibilidade no projeto devido à consideração de apenas um material, além do vazio, resultou em mecanismos onde a multiflexibilidade é praticamente inexistente.

Uma alternativa à atuação eletrotermomecânica estudada foi a atuação por fluxo de calor, que também possui aplicação prática. Mecanismos atuados por calor e compostos por dois e três materiais, além do vazio, foram obtidos, considerando ou não restrições de volume dos materiais.

Como sugestão de trabalho futuro, a implementação de restrições do acoplamento para o caso de mecanismos multiflexíveis poderia permitir a obtenção de mecanismos realmente funcionais, onde cada caso de carga resultaria num deslocamento diferente e facilmente distingüível. As restrições de acoplamento teriam um papel importante tanto na melhoria do índice de acoplamento no projeto com dois materiais, como na obtenção de multiflexibilidade no projeto com um único material, além do vazio.

A penalização de elementos com densidades intermediárias (escalas de cinza) também poderia ser implementada no futuro, para que as interpretações feitas sobre esse tipo de ocorrência pudessem ser reduzidas ou mesmo eliminadas. Essa penalização poderia contribuir para a eliminação de partes desconexas devido à existência de elementos com escala de cinza principalmente na interface entre diferentes materiais. 


\section{Referências}

ANANTHASURESH, G. K. Optimal synthesis methods for MEMS. Boston, USA: Kluwer Academic Publishers, 2003.

ANANTHASURESH, G. K.; KOTA, S.; GIANCHANDANI, Y. B. A methodical approach to the design of compliant micromechanisms. In: Technical Digest, Solid-State Sensor and Actuator Workshop. Hilton Head Island, SC, USA: [s.n.], 1994. p. 189-192.

ANSYS, INC. ANSYS, Inc. Theory Reference. Ansys release 6.0. [S.l.], October 2001 .

BATHE, K.-J. Finite Elements Procedures. New York, USA: Prentice Hall, 1995.

BECKERS, M. Topology optimization using a dual method with discrete variables. Structural Optimization, v. 17, n. 1, p. 14-24, 1999.

BENDS $\varnothing \mathrm{E}, \mathrm{M}$. P. Optimal shape design as a material distribution problem. Structural Optimization, v. 1, p. 192-202, 1989.

BENDSØE, M. P. Optimization of Structural Topology, Shape, and Material. Berlin, Germany: Springer-Verlag, 1995.

BENDSOE, M. P.; DIAZ, A.; KIKUCHI, N. Topology design of structures. In: . [S.l.]: Kluwer Academic Publishers, 1993. cap. Topology and generalized layout optimization of elastic structures, p. 159-205.

BENDSØE, M. P.; KIKUCHI, N. Generating optimal topologies in structural design using a homogenization method. Computer Methods in Applied Mechanics and Engineering, Elsevier, v. 71, n. 2, p. 197-224, November 1988.

BENDSØE, M. P.; RODRIGUES, H. C. Integrated topology and boundary shape optimization of 2-d solids. Computer Methods in Applied Mechanics and Engineering, v. 87, n. 1, p. 15-34, May 1991.

BENDSØE, M. P.; SIGMUND, O. Material interpolation schemes in topology optimization. Archive of Applied Mechanics, v. 69, n. 9-10, p. 635-654, 1999.

BENDSØE, M. P.; SIGMUND, O. Topology optimization: theory, methods and applications. In: Berlin, Germany: Springer, 2002. p. 71-152.

BOURDIN, B. Filters in topology optimization. International Journal for Numerical Methods in Engineering, v. 50, n. 9, p. 2143-2158, 2001.

BRUNS, T. E.; TORTORELLI, D. A. Topology optimization of non-linear elastic structures and compliant mechanisms. Computer Methods in Applied Mechanics and Engineering, Elsevier, v. 190, n. 26-27, p. 3443-3459, March 2001. 
BRUNS, T. E.; TORTORELLI, D. A. An element removal and reintroduction strategy for the topology optimization of structures and compliant mechanisms. International Journal for Numerical Methods in Engineering, Wiley, v. 57, n. 10, p. 1413-1430, May 2003.

BYUN, J.-K.; LEE, J.-H.; PARK, I.-H. Node-based distribution of material properties for topology optimization of electromagnetic devices. IEEE Transactions on Magnetics, v. 40, n. 2, p. 1212-1215, March 2004.

CANFIELD, S.; FRECKER, M. Topology optimization of compliant mechanical amplifiers for piezoelectric actuators. Structural and Multidisciplinary Optimization, Springer-Verlag, v. 20, n. 4, p. 269-279, December 2000.

CARDOSO, E. L. Controle de Complexidade na Otimização Topológica de Estruturas Contínuas. Dissertação (Mestrado) — Escola de Engenharia Universidade Federal do Rio Grande do Sul, Fevereiro 2000.

CARDOSO, E. L.; FONSECA, J. S. O. Intermediate density reduction and complexity control in the topology optimization. In: Iberian Latin-American Congress on Computational Methods in Engineering - CILAMCE. [S.l.: s.n.], 1999. v. 20.

CARDOSO, E. L.; FONSECA, J. S. O. Strain energy maximization approach to the design of fully compliant mechanisms using topology optimization. Latin American Journal of Solids and Structures, v. 1, p. 263-275, 2004.

CHEN, B. C.; SILVA, E. C. N.; KIKUCHI, N. Advances in computational design and optimization with application to mems. International Journal for Numerical Methods in Engineering, Wiley, v. 52, n. 1-2, p. 23-62, 2001.

CHEN, R. S.; KUNG, C.; LEE, G.-B. Analysis of the optimal dimension on the electrothermal microactuator. Journal of Micromechanics and Microengineering, Institute of Physics, v. 12, n. 3, p. 291-296, May 2002.

CHENG, K.-T. On non-smoothness in optimal design of solid, elastic plates. International Journal of Solids and Structures, v. 17, n. 8, p. 795-810, 1981.

CHENG, K.-T.; OLHOFF, N. Regularized formulation for optimal design of axisymmetric plates. International Journal of Solids and Structures, v. 18, n. 2, p. $153-169,1982$.

CHU, L. L.; GIANCHANDANI, Y. B. A micromachined 2d positioner with electrothermal actuation and sub-nanometer capacitive sensing. Journal of Micromechanics and Microengineering, Institute of Physics, v. 13, p. 279-285, 2003.

CHU, L. L.; NELSON, D.; OLIVER, A. D.; GIANCHANDANI, Y. B. Performance enhancement of polysilicon electrothermal microactuators by localized self-annealing. In: IEEE International Micro Electro Mechanical Systems Conference. [S.l.: s.n.], 2003. p. 68-71.

COMTOIS, J. H.; MICHALICEK, A.; BARRON, C. C. Electrothermal actuators fabricated in four-level planarized surface micromachined polycrystalline silicon. Sensors and Actuators A: Physical, Elsevier, v. 70, p. 23-31, 1998. 
COOK, R.; YOUNG, W. Advanced mechanics of materials. 1. ed. New york, USA: Macmillan, 1985.

COOK, R. D.; MALKUS, D. S.; PLESHA, M. E. Concepts and applications of finite element analysis. [S.l.]: John Wiley \& Sons, 1989.

COX, H. L. The design of structures of least weight. London, UK: Pergamon, 1956.

DANTZIG, G. Linear Programming and Extensions. [S.l.]: Princeton University Press, 1963.

DíAZ, A.; SIGMUND, O. Checkerboard patterns in layout optimization. Structural Optimization, v. 10, p. 40-45, 1995.

EJIMA, S.; NISHIWAKI, S.; SEKIGUCHI, M.; KIKUCHI, N. Optimal structural design of compliant mechanisms. JSME International Journal Series A: Solid Mechanics and Material Engineering, Japan Society of Mechanical Engineers, v. 43, n. 2, p. 130-137, April 2000.

FRECKER, M. I.; ANANTHASURESH, G. K.; NISHIWAKI, S.; KIKUCHI, N.; KOTA, S. Topological synthesis of compliant mechanisms using multi-criteria optimization. Journal of Mechanical Design, American Society of Mechanical Engineers, v. 119, n. 2, p. 238-245, June 1997.

FRECKER, M. I.; KIKUCHI, N.; KOTA, S. Topology optimization of compliant mechanisms with multiple outputs. Structural and Multidisciplinary Optimization, v. 17, n. 4, p. 269-278, 1999.

FUJII, D.; CHEN, B. C.; KIKUCHI, N. Composite material design of twodimensional structures using the homogenization design method. International Journal for Numerical Methods in Engineering, v. 50, n. 9, p. 2031-2051, 2001.

GIBIANSKY, L. V.; SIGMUND, O. Multiphase composites with extremal bulk modulus. Journal of the Mechanics and Physics of Solids, v. 48, n. 3, p. 461-498, 2000 .

HABER, R. B.; JOG, C. S.; BENDSØE, M. P. A new approach to variable-topology shape design using a constraint on the perimeter. Structural Optimization, v. 11, p. 1-12, 1996.

HAFTKA, R. T.; GURDAL, Z.; KAMAT, M. P. Elements of Structural Optimization. 2. ed. [S.l.]: Kluwer Academic Publishers, 1990.

HANSON, R. J.; HIEBERT, K. L. Subroutine DSPLP - Solve linear programming problems involving at most a few thousand constraints and variables. Takes advantage of sparsity in the constraint matrix. Part of SLATEC Common Mathematical Library, Version 4.1. May 1992. Acessado em janeiro de 2005. Disponível em: <http://www.netlib.org/slatec/src/dsplp.f>.

HASHIN, Z.; SHTRIKMAN, S. A variational approach to the theory of the elastic behaviour of multiphase materials. Journal of the Mechanics and Physics of Solids, v. 11, n. 2, p. 127-140, March-April 1963. 
HASSANI, B.; HINTON, E. A review of homogenization and topology optimization iii - topology optimization using optimality criteria. Computers $\mathscr{6}$ Structures, v. 69, n. 6, p. 739-756, December 1998.

HEMP, W. S. Optimum structures. [S.l.]: Clarendon Press, 1973. (Oxford engineering science series).

HETRICK, J. A.; KIKUCHI, N.; KOTA, S. Robustness of compliant mechanism topology optimization formulations. In: VARADAN, V. V. (Ed.). Proceedings of SPIE. [S.l.: s.n.], 1999. (Smart Structures and Materials 1999: Mathematics and Control in Smart Structures, v. 3667), p. 244-254.

HICKEY, R.; SAMEOTO, D.; HUBBARD, T.; KUJATH, M. Time and frequency response of two-arm micromachined thermal actuators. Journal of Micromechanics and Microengineering, Institute of Physics, v. 13, n. 1, p. 40-46, January 2003.

HOWELL, L. L. Compliant Mechanisms. New York, USA: John Wiley \& Sons, Inc., 2001.

ISHIHARA, H.; ARAI, F.; FUKUDA, T. Micro mechatronics and micro actuators. IEEE/ASME Transactions on Mechatronics, v. 1, n. 1, p. 68-79, 1996.

JENSEN, J. S.; SIGMUND, O. Systematic design of photonic crystal structures using topology optimization: Low-loss waveguide bends. Applied Physics Letters, American Institute of Physics, v. 84, n. 12, p. 2022-2024, March 2004.

JOG, C. S.; HABER, R. B. Stability of finite element models for distributedparameter optimization and topology design. Computer Methods in Applied Mechanics and Engineering, v. 130, n. 3-4, p. 203-226, April 1996.

JOG, C. S.; HABER, R. B.; BENDSØE, M. P. Topology design of structures. In: _ [S.l.]: Kluwer Academic Publishers, 1993. cap. A displacement based topology design with optimized self-adaptive materials, p. 219-238.

JONSMANN, J. Technology Development for Topology Optimised Thermal Microactuators. Tese (Doutorado) — Mikroelektronik Centret - Technical University of Denmark, Lyngby, Denmark, December 1999.

JONSMANN, J.; SIGMUND, O.; BOUWSTRA, S. Compliant electro-thermal microactuators. In: Twelfth IEEE International Conference on Micro Electro Mechanical Systems. Orlando, FL, USA: [s.n.], 1999. p. 588-593.

JONSMANN, J.; SIGMUND, O.; BOUWSTRA, S. Compliant thermal microactuators. Sensors and Actuators A: Physical, Elsevier, v. 76, n. 1-3, p. 463-469, August 1999.

KIKUCHI, N.; HOLLISTER, S.; YOO, J. A concept of image-based integrated cae for production engineering. In: JAPAN SOCIETY OF MECHANICAL ENGINEERS. Proceedings of International Symposium on Optimization and Innovative Design. Tokyo, Japan, 1997. p. 75-90.

KOHN, R. V.; STRANG, G. Optimal design and relaxation of variational problems, i. Communications on Pure and Applied Mathematics, v. 39, p. 113-137, 1986. 
KOHN, R. V.; STRANG, G. Optimal design and relaxation of variational problems, ii. Communications on Pure and Applied Mathematics, v. 39, p. 139-182, 1986.

KOHN, R. V.; STRANG, G. Optimal design and relaxation of variational problems, iii. Communications on Pure and Applied Mathematics, v. 39, p. 353-377, 1986.

KOTA, S.; HETRICK, J.; LI, Z.; SAGGERE, L. Tailoring unconventional actuators using compliant transmissions: design methods and applications. IEEE/ASME Transactions on Mechatronics, v. 4, n. 4, p. 396-408, 2000.

KOTA, S.; JOO, J.; LI, Z.; RODGERS, S. M.; SNIEGOWSKI, J. Design of compliant mechanisms: applications to mems. Analog Integrated Circuits and Signal Processing, Springer Science+Business, v. 29, n. 1-2, p. 7-15, October 2001 .

LARSEN, U. D.; SIGMUND, O.; BOUWSTRA, S. Design and fabrication of compliant mechanisms and material structures with negative poisson's ratio. Journal of Microelectromechanical Systems, IEEE, v. 6, n. 2, p. 99-106, June 1997.

LAU, G. K.; DU, H.; LIM, M. K. Techniques to suppress intermediate density in topology optimization of compliant mechanisms. Computational Mechanics, Springer-Verlag, v. 27, n. 5, p. 426-435, May 2001.

LAU, G. K.; DU, H.; LIM, M. K. Use of functional specifications as objective functions in topological optimization of compliant mechanism. Computer Methods in Applied Mechanics and Engineering, Elsevier, v. 190, n. 34, p. 4421-4433, May 2001.

LERCH, P.; SLIMANE, C. K.; ROMANOWICZ, B.; RENAUD, P. Modelization and characterization of asymmetrical thermal micro-actuators. Journal of Micromechanics and Microengineering, Institute of Physics, v. 6, n. 1, p. 134-137, March 1996.

LIMA, C. R.; SILVA, E. C. N. Projeto de mecanismos flexíveis usando otimização topológica. In: Anais do XVI COBEM (Congresso Brasileiro de Engenharia Mecânica). São Paulo, SP, Brasil: [s.n.], 2001.

LIMA, C. R. de. Projeto de mecanismos flexíveis usando o método de otimização topológica. Dissertação (Mestrado) — Escola Politécnica, Universidade de São Paulo, 2002.

LURIE, K. A.; CHERKAEV, A. V.; FEDOROV, A. V. Regularization of optimal design problems for bars and plates i. Journal of Optimization Theory and Applications, v. 37, p. 499-522, 1982a.

LURIE, K. A.; CHERKAEV, A. V.; FEDOROV, A. V. Regularization of optimal design problems for bars and plates ii. Journal of Optimization Theory and Applications, v. 37, p. 523-543, 1982b.

MA, Z.-D.; KIKUCHI, N.; CHENG, H.-C. Topological design for vibrating structures. Computer Methods in Applied Mechanics and Engineering, v. 121, n. 1-4, p. 259-280, March 1995. 
MANKAME, N. D.; ANANTHASURESH, G. K. Comprehensive thermal modelling and characterization of an electro-thermal-compliant microactuator. Journal of Micromechanics and Microengineering, Institute of Physics, v. 11, p. 452-462, 2001.

MICHELL, A. G. M. The limits of economy of material in frame structures. Philosophical Magazine, Series 6, v. 8, p. 589-597, 1904.

MLEJNEK, H. P. Some aspects of the genesis of structures. Structural Optimization, v. 5, p. 64-69, 1992.

MLEJNEK, H. P.; SCHIRRMACHER, R. An engineer's approach to optimal material distribution and shape finding. Computer Methods in Applied Mechanics and Engineering, v. 106, p. 1-26, 1993.

MOULTON, T.; ANANTHASURESH, G. K. Micromechanical devices with embedded electro-thermal-compliant actuation. Sensors and Actuators A: Physical, Elsevier, v. 90, n. 1-2, p. 38-48, May 2001.

MURAT, F.; TARTAR, L. Calcul des variations et homogénéisation. In: BERGMAN, D. (Ed.). Les méthodes de l'homogénéisation: théorie et applications en physique. Paris, France: Eyrolles, 1985. (Collection de la direction des études et recherches d'electricité de France, v. 57), p. 319-369.

NISHIWAKI, S.; FRECKER, M. I.; MIN, S.; KIKUCHI, N. Topology optimization of compliant mechanisms using the homogenization method. International Journal for Numerical Methods in Engineering, Wiley, v. 42, p. 535-559, 1998.

NISHIWAKI, S.; MIN, S.; YOO, J.; KIKUCHI, N. Optimal structural design considering flexibility. Computer Methods in Applied Mechanics and Engineering, Elsevier, v. 190, p. 4457-4504, 2001.

OLHOFF, N.; BENDSØE, M. P.; RASMUSSEN, J. On cad-integrated structural topology and design optimization. Computer Methods in Applied Mechanics and Engineering, v. 89, n. 1-3, p. 259-279, August 1991.

OLHOFF, N.; KOG, L.; THOMSEN, J. Bi-material topology optimization. In: KERSKOVITES, J. (Ed.). Structural Optimization. Rio de Janeiro, Brazil, 1993. p. $327-334$.

OWEN, J. B. B. The analysis and design of light structures. London, UK: Edward Arnold, 1965.

PARK, J. S.; CHU, L. L.; SIWAPORNSATHAIN, E.; GIANCHANDANI, Y. B. Long throw and rotary output electro-thermal actuators based on bent-beam suspensions. In: IEEE International Micro Electro Mechanical Systems Conference. Japan: [s.n.], 2000. p. 680-685.

PEDERSEN, N. L. On topology optimization of plates with prestress. International Journal for Numerical Methods in Engineering, Wiley, v. 51, p. 225-239, 2001.

PETERSEN, K. E. Silicon as mechanical material. In: Proceedings of IEEE. [S.l.: s.n.], 1982. v. 70, p. 420-457. 
PETERSSON, J.; SIGMUND, O. Slope constrained topology optimization. International Journal for Numerical Methods in Engineering, v. 41, n. 8, p. 1417-1434, 1998.

PRAGER, W. A note on discretized michell structures. Computer Methods in Applied Mechanics and Engineering, Elsevier, v. 3, n. 3, p. 349-355, May 1974.

PRESS, W. H.; FLANNERY, B. P.; TEUKOLSKY, S. A.; VETTERLING, W. T. Numerical Recipes in $C$ : The Art of Scientific Computing. [S.l.]: Cambridge University Press, 1999.

QI, H.; KIKUCHI, N.; MAZUMDER, J. Interface study and boundary smoothing on designed composite material microstructures for manufacturing purposes. Structural and Multidisciplinary Optimization, v. 26, n. 5, p. 326-332, 2004 .

QUE, L.; OTRADOVEC, L.; OLIVER, A. D.; GIANCHANDANI, Y. B. Pulse and dc operation lifetimes of bent-beam electrothermal actuators. In: The 14th IEEE International Conference on Micro Electro Mechanical Systems. Interlaken, Switzerland: [s.n.], 2001. p. 570-573.

QUE, L.; PARK, J. S.; GIANCHANDANI, Y. B. Bent-beam electro-thermal actuators for high force applications. In: IEEE International Micro Electro Mechanical Systems Conference. Orlando, FL, USA: [s.n.], 1999. p. 31-36.

QUE, L.; PARK, J.-S.; GIANCHANDANI, Y. B. Bent-beam electrothermal actuators - part i: Single beam and cascaded devices. Journal of Microelectromechanical Systems, IEEE, v. 10, n. 2, p. 247-254, June 2001.

QUE, L.; PARK, J.-S.; LI, M.-H.; GIANCHANDANI, Y. B. Reliability studies of bent-beam electro-thermal actuators. In: Proceedings of 38th Annual IEEE International Reliability Physics Symposium. San Jose, CA, USA: [s.n.], 2000. p. 118-122.

RAI-CHOUDHURY, P. MEMS and MOEMS Technology and Applications. Bellingham, Washington, USA: SPIE Press, 2000.

RAĬTUM, U. Ë. On optimal control problems for linear elliptic equations. Soviet Mathematics Doklady, v. 20, p. 129-132, 1979.

REID, J. R.; BRIGHT, V. M.; COMTOIS, J. H. Force measurements of polysilicon thermal microactuators. In: Proceedings of SPIE. [S.l.: s.n.], 1996. (Micromachined Devices and Components II 1996, v. 2882), p. 296-306.

REYNAERTS, D.; PEIRS, J.; BRUSSEL, H. V. A mechatronic approach to microsystem design. IEEE/ASME Transactions on Mechatronics, v. 3, n. 1, p. 24-33, 1998.

RODRIGUES, H.; SOTO, C. A.; TAYLOR, J. E. A design model to predict optimal two-material composite structures. Structural and Multidisciplinary Optimization, Springer-Verlag, v. 17, n. 2-3, p. 186-198, April 1999.

RODRIGUES, H. C.; FERNANDEZ, P. Topology design of structures. In: . [S.l.]: Kluwer Academic Publishers, 1993. cap. Topology optimization of linear elastic structures subjected to thermal loads, p. 437-450. 
RODRIGUES, H. C.; FERNANDEZ, P. A material based model for topology optimization of thermoelastic strutures. International Journal for Numerical Methods in Engineering, Wiley, v. 38, n. 12, p. 1951-1965, 1995.

ROZVANY, G. I. N. Aims, scope, methods, history and unified terminology of computer-aided topology optimization in structural mechanics. Structural and Multidisciplinary Optimization, v. 21, n. 2, p. 90-108, April 2001.

SHIGLEY, J. E.; MISCHKE, C. R. Mechanical Engineering Design. 5th edition. ed. [S.l.]: McGraw-Hill, 1989.

SIGMUND, O. Some inverse problems in topology design of materials and mechanisms. In: BESTLE, D.; SCHIELEN, W. (Ed.). IUTAM Symposium on Optimization of Mechanical Systems. Netherlands: Kluwer Academic Publishers, 1996. p. 277-284.

SIGMUND, O. On the design of compliant mechanisms using topology optimization. Mechanics of Structures and Machines, v. 25, n. 4, p. 495-526, 1997.

SIGMUND, O. Systematic design of microactuators using topology optimization. In: VARADAN, V. K.; MCWHORTER, P. J.; SINGER, R. A.; VELLEKOOP, M. J. (Ed.). Proceedings of SPIE. [S.l.: s.n.], 1998. (Smart Structures and Materials 1998: Smart Electronics and MEMS, v. 3328), p. 23-31.

SIGMUND, O. On the optimality of bone microstructure. In: PEDERSEN, P.; BENDSØE, M. P. (Ed.). IUTAM Symposium on Synthesis in Bio Solid Mechanics. Lyngby, Denmark: Kluwer Academic Publishers, 1999. p. 221-234.

SIGMUND, O. Topology optimization: a tool for the tailoring of structures and materials. A special issue of the Philosophical Transactions of the Royal Society: Science into the next Millennium (Issue III, Mathematics, Physics and Engineering), v. 358, n. 1765, p. 211-228, 2000.

SIGMUND, O. A 99 line topology optimization code written in matlab. Structural and Multidisciplinary Optimization, v. 21, n. 2, p. 120-127, 2001.

SIGMUND, O. Design of multiphysics actuators using topology optimization part i: One-material structures. Computer Methods in Applied Mechanics and Engineering, Elsevier, v. 190, p. 6577-6604, 2001.

SIGMUND, O. Design of multiphysics actuators using topology optimization part ii: Two-material structures. Computer Methods in Applied Mechanics and Engineering, Elsevier, v. 190, p. 6605-6627, 2001.

SIGMUND, O.; PETERSSON, J. Numerical instabilities in topology optimization: A survey on procedures dealing with checkerboards, meshdependencies and local minima. Structural and Multidisciplinary Optimization, v. 16, n. 1, p. 68-75, July 1998.

SIGMUND, O.; TORQUATO, S. Composites with extremal thermal expansion coefficients. Applied Physics Letters, American Institute of Physics, v. 69, n. 21, p. 3203-3205, November 1996. 
SIGMUND, O.; TORQUATO, S. Design of materials with extreme thermal expansion using a three-phase topology optimization method. Journal of the Mechanics and Physics of Solids, v. 45, n. 6, p. 1037-1067, 1997.

SIGMUND, O.; TORQUATO, S.; AKSAY, I. A. On the design of 1-3 piezocomposites using topology optimization. Journal of Materials Research, Materials Research Society, v. 13, n. 4, p. 1038-1048, April 1998.

SILVA, E. C. N.; FONSECA, J. S. O.; KIKUCHI, N. Optimal design of periodic piezocomposites. Computer Methods in Applied Mechanics and Engineering, v. 159, n. 1-2, p. 49-77, 1998.

SILVA, E. C. N.; NISHIWAKI, S.; FONSECA, J. S. O.; KIKUCHI, N. Optimization methods applied to material and flextensional actuator design using the homogenization method. Computer Methods in Applied Mechanics and Engineering, v. 172, n. 1-4, p. 241-271, April 1999.

SILVA, E. C. N.; NISHIWAKI, S.; KIKUCHI, N. Topology optimization design of flextensional actuators. IEEE Transactions on Ultrasonics, Ferroelectrics, and Frequency Control, v. 47, n. 3, p. 657-671, 2000.

SUZUKI, K.; KIKUCHI, N. A homogenization method for shape and topology optimization. Computer Methods in Applied Mechanics and Engineering, v. 93, n. 3, p. 291-318, December 1991.

SVANBERG, K. The method of moving asymptotes - a new method for structural optimization. International Journal of Numerical Methods in Engineering, v. 24, p. 359-373, 1987.

SVANBERG, K. Global convergence of the stress ratio method for truss sizing. Structural Optimization, v. 8, p. 60-68, 1994.

SVANBERG, K. On the convexity and concavity of compliances. Structural Optimization, v. 7, p. 42-46, 1994.

SVANBERG, K.; WERME, M. A hierarchical neighbourhood search method for topology optimization. Structural and Multidisciplinary Optimization, v. 29, n. 5 , p. 325-340, 2005.

SWAN, C. C.; KOSAKA, I. Voigt-reuss topology optimization for structures with linear elastic material behaviours. International Journal for Numerical Methods in Engineering, v. 40, n. 16, p. 3033-3057, 1997.

SYMS, R. R. A. Electrothermal frequency tuning of folded and coupled vibrating micromechanical resonators. Journal of Microelectromechanical Systems, IEEE, v. 7, n. 2 , p. 164-171, June 1998.

TAI, K.; CHEE, T. H. Design of structures and compliant mechanism by evolutionary optimization of morphological representations of topology. Journal of Mechanical Design, American Society of Mechanical Engineers, v. 122, n. 4, p. 560-566, December 2000.

THOMAS, H. L.; VANDERPLAATS, G. N.; SHYY, Y. K. A study of move limit adjustment strategies in the approximation concepts approach to structural synthesis. In: Proceedings of the Fourth AIAA/USAF/- NASA/OAI Symposium 
on Multidisciplinary Analysis and Optimization. Cleveland, OH, USA: [s.n.], 1992. p. 507-512.

THOMSEN, J. Topology optimization of structures composed of one or two materials. Structural Optimization, v. 5, p. 108-115, 1992.

TORQUATO, S. Modeling of physical properties of composite materials. International Journal of Solids and Structures, v. 37, n. 1-2, p. 411-422, 2000.

WANG, M. Y.; WANG, X. "color" level sets: a multi-phase method for structural topology optimization with multiple materials. Computer Methods in Applied Mechanics and Engineering, Elsevier, v. 193, p. 469-496, 2004.

WANG, M. Y.; WANG, X. A level-set based variational method for design and optimization of heterogeneous objects. Computer-Aided Design, Elsevier, v. 37, p. 321-337, 2005.

WANG, S.; KANG, J.; NOH, J. Topology optimization of a single-phase induction motor for rotary compressor. IEEE Transactions on Magnetics, v. 40, n. 3, p. 1591-1596, May 2004.

YAN, D.; KHAJEPOUR, A.; MANSOUR, R. Modeling of two-hot-arm horizontal thermal actuator. Journal of Micromechanics and Microengineering, Institute of Physics, v. 13, n. 2, p. 312-322, March 2003.

YIN, L.; ANANTHASURESH, G. K. Topology optimization of compliant mechanisms with multiple materials using a peak function material interpolation scheme. Structural and Multidisciplinary Optimization, Springer-Verlag, v. 23, n. 1, p. 49-62, December 2001.

YIN, L.; ANANTHASURESH, G. K. A novel topology design scheme for the multi- physics problems of electro-thermally actuated compliant micromechanisms. Sensors and Actuators A: Physical, Elsevier, v. 97-98, p. 599-609, 2002.

YIN, L.; YANG, W. Topology optimization for tunnel support in layered geological structures. International Journal for Numerical Methods in Engineering, v. 47, n. 12, p. 1983-1996, 2000.

YOO, J.; KIKUCHI, N. Topology optimization in magnetic fields using the homogenization design method. International Journal for Numerical Methods in Engineering, Wiley, v. 48, n. 10, p. 1463-1479, 2000.

YULIN, M.; XIAOMING, W. A level set method for structural topology optimization and its applications. Advances in Engineering Software, Elsevier, v. 35 , p. $415-441,2004$.

ZHOU, M.; ROZVANY, G. I. N. The coc algorithm, part ii: Topological, geometrical and generalized shape optimization. Computer Methods in Applied Mechanics and Engineering, Elsevier, v. 89, n. 1-3, p. 309-336, August 1991.

ZIENKIEWICZ, O.; TAYLOR, R. Finite element method: Solid and fluid mechanics dynamics and non-linearity. 4. ed. [S.l.]: McGraw-Hill, 1991. 


\section{Apêndice A - Imposição de condições de contorno de Dirichlet}

Dado um domínio a modelado por elementos finitos, tem-se:

$$
\begin{gathered}
\boldsymbol{K}_{i}(\rho) U_{i}(\rho)=\boldsymbol{P}_{i}\left(U_{i-1}(\rho), \rho\right) \\
\boldsymbol{P}_{i}\left(U_{i-1}(\rho), \rho\right)=P_{i C}(\rho)+P_{i D}\left(U_{i-1}(\rho), \rho\right)
\end{gathered}
$$

onde $\boldsymbol{P}_{\boldsymbol{i} C}$ é a carga nodal concentrada e $\boldsymbol{P}_{\boldsymbol{i} D}$ é a carga distribuída, calculada com uma integração sobre a área ou volume dos elementos.

Ao impor um valor $\bar{U}_{i_{j}}$ a um dado grau de liberdade $j$ de $\boldsymbol{U}_{\boldsymbol{i}}$, também conhecida como condição de contorno de Dirichlet (BATHE, 1995), a matriz $\boldsymbol{K}_{\boldsymbol{i}}$ e o vetor de carga $\boldsymbol{P}_{\boldsymbol{i}}$ são substituídos pela matriz $\boldsymbol{K}_{\boldsymbol{i}}^{*}$ e vetor de carga $\boldsymbol{P}_{\boldsymbol{i}}^{*}$, respectivamente. Estas são versões modificadas de $\boldsymbol{K}_{\boldsymbol{i}}$ e $\boldsymbol{P}_{\boldsymbol{i}}$ da seguinte maneira: a linha $j$ da matriz $\boldsymbol{K}_{i}^{*}$ é feita nula, exceto na coluna $j$ onde o elemento é unitário. O valor da linha $j$ de $\boldsymbol{P}_{i}^{*}$ é alterado para $\bar{U}_{i_{j}}$, de modo a forçar o dado valor para o grau de liberdade de $j$ de $\boldsymbol{U}_{\boldsymbol{i}}$. Para manter a simetria da matriz $\boldsymbol{K}_{i}^{*}$, em todas as linhas dessa matriz (exceto a $j$ ) a coluna $j$ é zerada, e em cada linha do vetor de carga $\boldsymbol{P}_{\boldsymbol{i}}^{*}$ (com exceção da linha $j$ ) é subtraído o produto dos valores do elemento da coluna $j$ de $\boldsymbol{K}_{\boldsymbol{i}}$ e $\overline{\boldsymbol{U}}_{\boldsymbol{i}_{j}}$. Assim, o sistema a ser resolvido é dado por:

$$
\boldsymbol{K}_{i}^{*}(\rho) \boldsymbol{U}_{i}(\rho)=\boldsymbol{P}_{i}^{*}\left(U_{i-1}(\rho), \rho\right)
$$

Exemplificando para um sistema com três graus de liberdade e condição de contorno de Dirichlet no primeiro grau de liberdade $(j=1)$, ficaria:

$$
\underbrace{\left[\begin{array}{ccc}
1 & 0 & 0 \\
0 & K_{i_{22}} & K_{i_{23}} \\
0 & K_{i_{32}} & K_{i_{33}}
\end{array}\right]}_{\boldsymbol{K}_{\boldsymbol{i}}^{*}} \underbrace{\left\{\begin{array}{l}
U_{i_{1}} \\
U_{i_{2}} \\
U_{i_{3}}
\end{array}\right\}}_{\boldsymbol{U}_{\boldsymbol{i}}}=\underbrace{\left\{\begin{array}{c}
\bar{U}_{i_{1}} \\
P_{i_{2}}-K_{i_{21}} \bar{U}_{i_{1}} \\
P_{i_{3}}-K_{i_{31}} \bar{U}_{i_{1}}
\end{array}\right\}}_{\boldsymbol{P}_{\boldsymbol{i}}^{*}}
$$

onde $K_{i_{m n}}$ é o elemento da linha $m$ e coluna $n$ da matriz $\boldsymbol{K}_{\boldsymbol{i}}, U_{i_{m}}$ é o grau de 
liberdade $m$ do vetor $\boldsymbol{U}_{\boldsymbol{i}}$ e $P_{i_{m}}$ é a carga sobre o grau de liberdade $m$ do vetor $\boldsymbol{P}_{\boldsymbol{i}}$.

O mesmo conceito pode ser estendido para a imposição de mais de um grau de liberdade. Com a imposição de valores para o primeiro e segundo graus de liberdade em um sistema com quatro graus de liberdade, tem-se:

$$
\underbrace{\left[\begin{array}{cccc}
1 & 0 & 0 & 0 \\
0 & 1 & 0 & 0 \\
0 & 0 & K_{i_{33}} & K_{i_{34}} \\
0 & 0 & K_{i_{43}} & K_{i_{44}}
\end{array}\right]}_{\boldsymbol{K}_{\boldsymbol{i}}^{*}} \underbrace{\left\{\begin{array}{l}
U_{i_{1}} \\
U_{i_{2}} \\
U_{i_{3}} \\
U_{i_{4}}
\end{array}\right\}}_{\boldsymbol{U}_{\boldsymbol{i}}}=\underbrace{\left\{\begin{array}{c}
\bar{U}_{i_{1}} \\
\bar{U}_{i_{2}} \\
P_{i_{3}}-K_{i_{31}} \bar{U}_{i_{1}}-K_{i_{32}} \bar{U}_{i_{2}} \\
P_{i_{4}}-K_{i_{41}} \bar{U}_{i_{1}}-K_{i_{42}} \bar{U}_{i_{2}}
\end{array}\right\}}_{\boldsymbol{P}_{\boldsymbol{i}}^{*}}
$$




\section{Apêndice B - Verificações da Implementação}

\section{B.1 Verificação do Método de Elementos Finitos (MEF)}

Para verificar o MEF implementado, foi feito um modelo com quatro elementos e dois materiais. A figura B.1 ilustra o modelo usado, com a identificação dos nós, materiais e condições de contorno.

Nos pontos 1 e 2, além das condições de contorno elásticas (restrição em $x$ e $y$ ), foram aplicadas as temperaturas $T$ (nulas - note que as temperaturas obtidas são as diferenças em relação à temperatura ambiente). No ponto 1 , foi aplicada a corrente de entrada $i$ (iguais a $1 \mathrm{~A}$ ) e no ponto 2, a tensão de referência. A corrente elétrica aplicada entra pelo ponto 1 e sai pelo ponto 2. A espessura dos elementos $t^{e}$ é uniforme e vale $15 \times 10^{-3} \mathrm{~mm}$. Cada elemento tem altura e largura de $1 \mathrm{~mm}$. As propriedades do material 1 e 2 correspondem, respectivamente, ao níquel e cobre. Na tabela B.1 estão os valores das propriedades consideradas, com a unidade de comprimento utilizada sendo o $\mathrm{mm}$, o que ameniza algum mal-condicionamento numérico.

Tabela B.1: Propriedades dos Materiais - Níquel e Cobre.

\begin{tabular}{|l|c|c|}
\hline Propriedades & Material 1 (Níquel) & Material 2 (Cobre) \\
\hline \hline Módulo de Elasticidade E $\left(\mathrm{N} / \mathrm{mm}^{2}\right)$ & $188 \times 10^{3}$ & $110 \times 10^{3}$ \\
Coeficiente de Poisson & 0,31 & 0,31 \\
Coeficiente de Expansão Térmica $\left(\mathrm{K}^{-1}\right)$ & $15 \times 10^{-6}$ & $16,5 \times 10^{-6}$ \\
Condutividade Elétrica $(1 / \Omega \cdot \mathrm{mm})$ & $6,37 \times 10^{3}$ & $5,977 \times 10^{4}$ \\
Condutividade Térmica $(\mathrm{W} / \mathrm{K} \cdot \mathrm{mm})$ & $90,7 \times 10^{-3}$ & $401 \times 10^{-3}$ \\
\hline
\end{tabular}

Para a simulação em ANSYS deste modelo, foi usado o elemento de quatro nós SHELL157, para as análises elétricas e eletrotérmicas, e o PLANE42, para a análise termoelástica. As análises elétricas e eletrotérmicas foram feitas separadamente das termoelásticas. Portanto, apesar do elemento SHELL157 


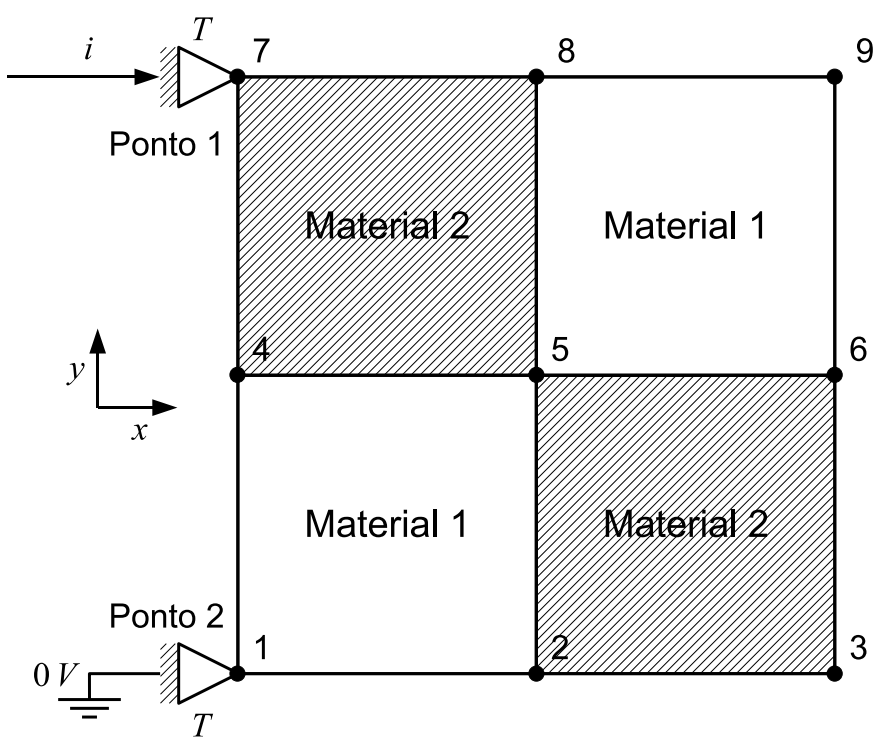

Figura B.1: Modelo para verificação do MEF - com indicação dos nós, materiais e condições de contorno.

possuir graus de liberdade elásticos tridimensionais (deslocamento no eixo Z e rotações em torno de $x$ e $y$ ), não foi necessário restringí-los porque esse elemento não foi usado na análise termoelástica. Assim, os resultados do ANSYS e do programa em C podem ser vistos nas figuras B.2 e B.3, e nas tabelas B.2 a B.7. Note que, para estes resultados, não foi considerada a conveç̧ão nos elementos.

Tabela B.2: Resultados da Análise Elétrica - Tensões Elétrica.

\begin{tabular}{|c|c|c|}
\hline Nó & Programa em C (V) & ANSYS (V) \\
\hline \hline 1 & 0,0000000 & 0,000000 \\
2 & 0,0152397 & 0,015240 \\
3 & 0,0155710 & 0,015571 \\
4 & 0,0160217 & 0,016022 \\
5 & 0,0157665 & 0,015766 \\
6 & 0,0155113 & 0,015511 \\
7 & 0,0176351 & 0,017635 \\
8 & 0,0162933 & 0,016293 \\
9 & 0,0158344 & 0,015834 \\
\hline
\end{tabular}

Pode-se verificar que as diferenças entre os resultados do programa em C e do ANSYS, em todas as análises, são menores que 0,002\% (poderiam ser até menores caso os resultados do ANSYS fossem obtidos com mais casas decimais). Os valores nodais das densidades de corrente não puderam ser comparados diretamente com o resultado obtido pelo ANSYS, por este não listar esse tipo de dado (apenas por elemento), mas pode-se verificar que os valores listados por elemento são aproximadamente os verificados no centro de cada um deles através da figura B.3(b). As tensões de von Mises, apesar de também não serem listadas pelo ANSYS, podem ser verificadas comparando-se as figuras B.2(f) e B.3(f). Neste 


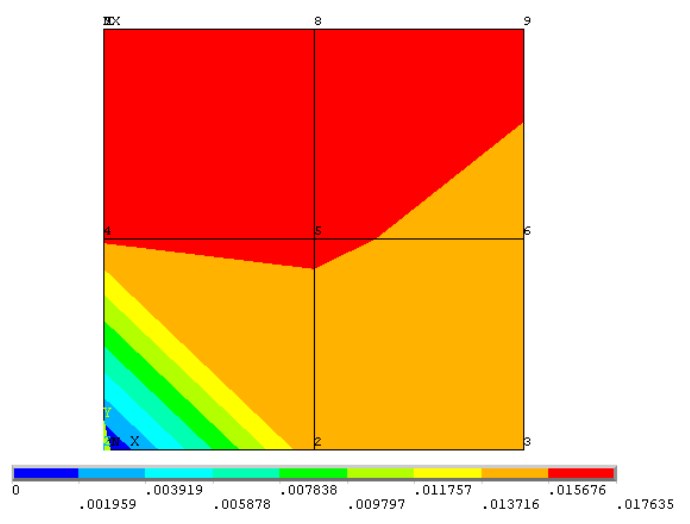

(a) Tensões Elétricas

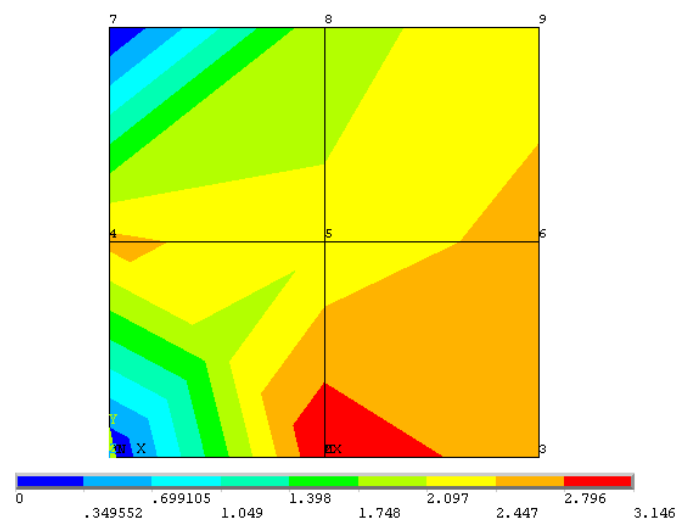

(c) Diferenças de Temperatura

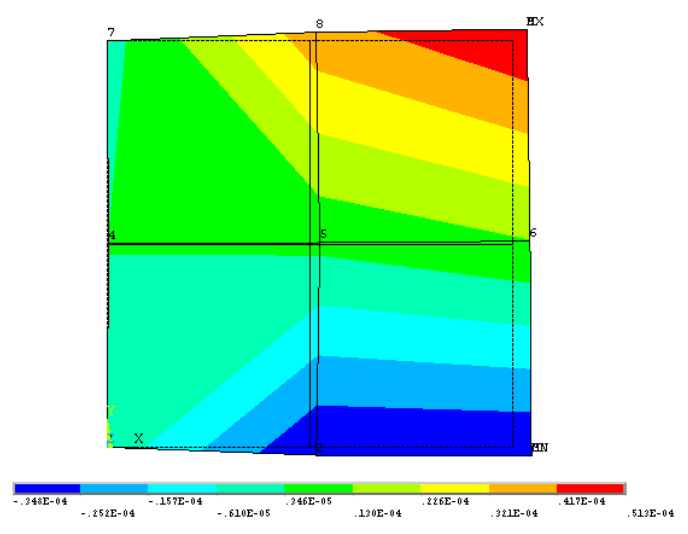

(e) Deslocamento em $y$

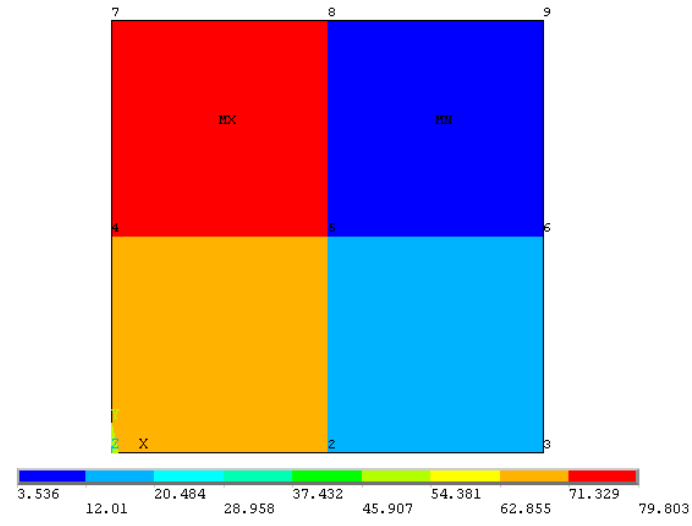

(b) Densidades de Corrente

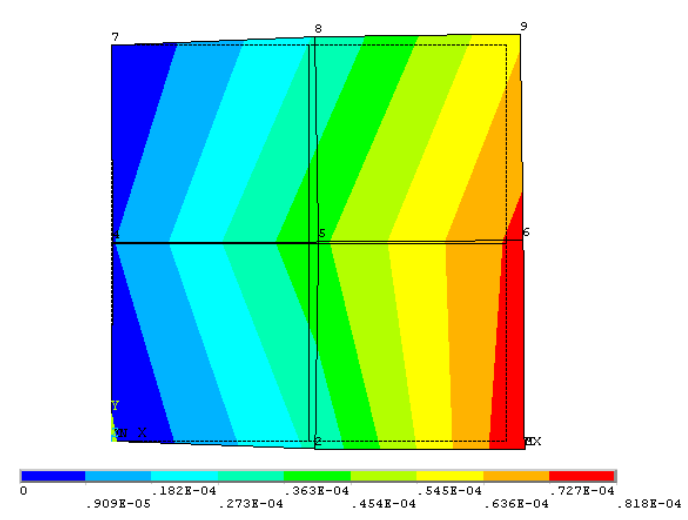

(d) Deslocamento em $x$

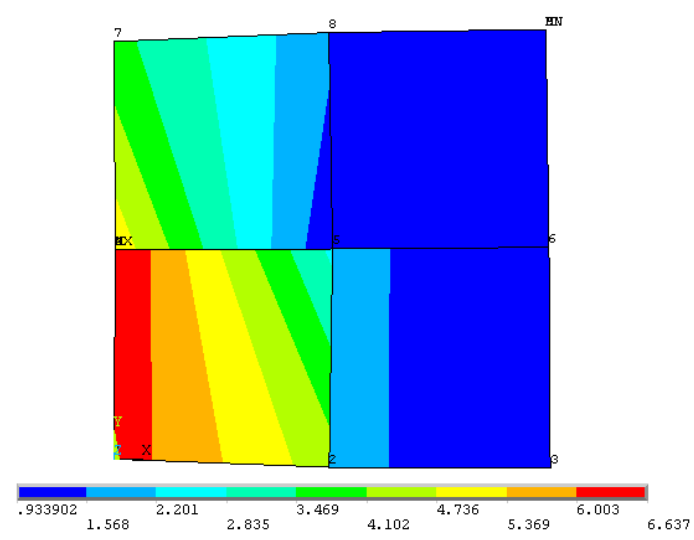

(f) Tensões de von Mises

Figura B.2: Resultados das Análises por ANSYS (sem convecção). 


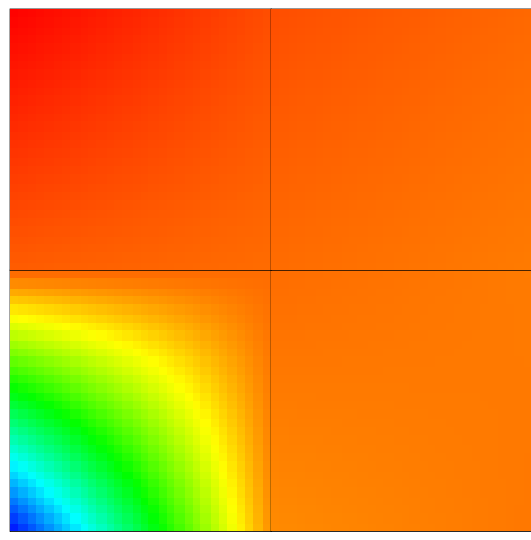

(a) Tensões Elétricas
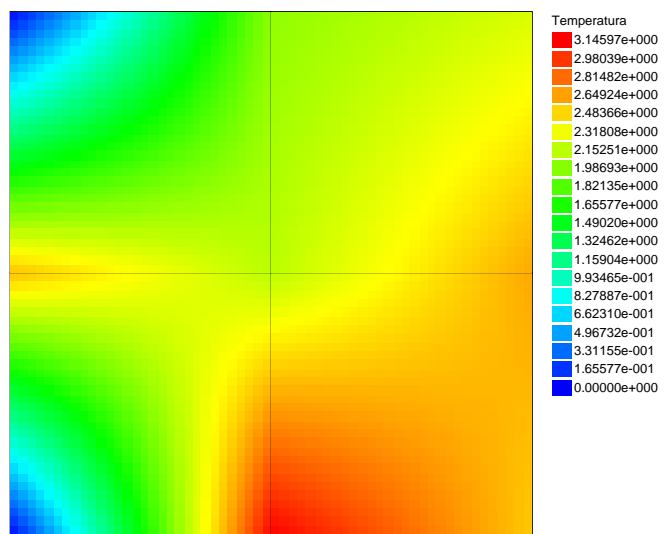

(c) Diferenças de Temperatura

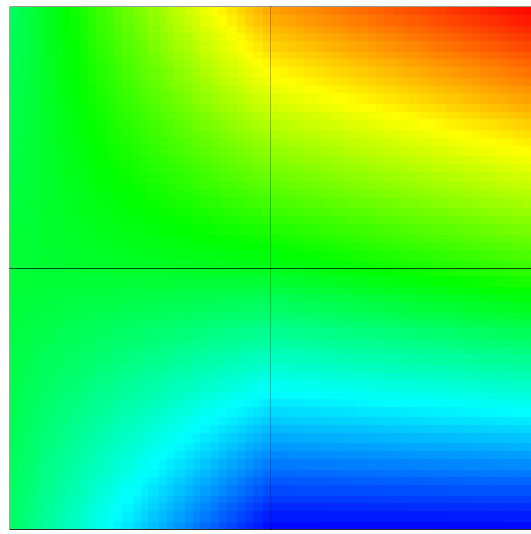

(e) Deslocamento em $y$
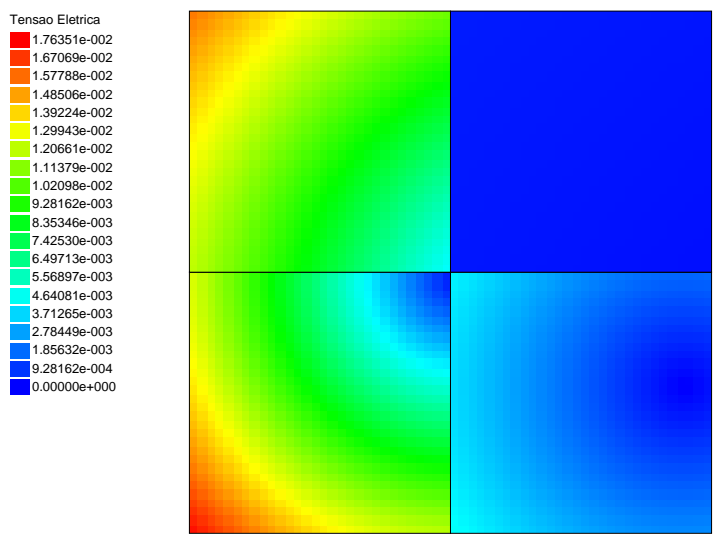

(b) Densidades de Corrente
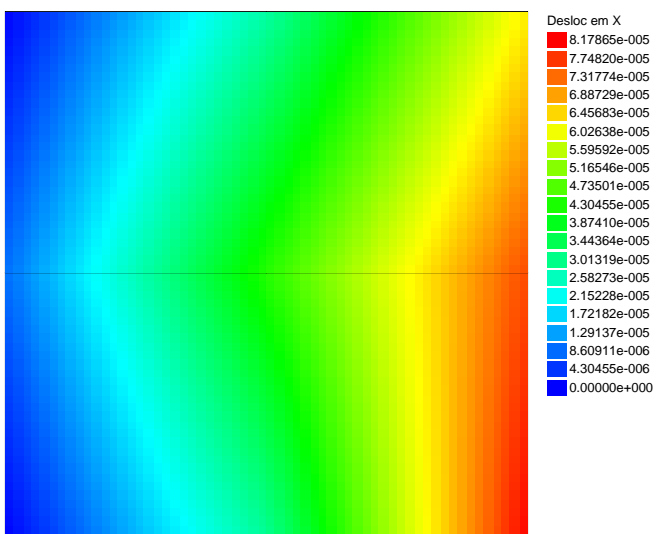

(d) Deslocamento em $x$
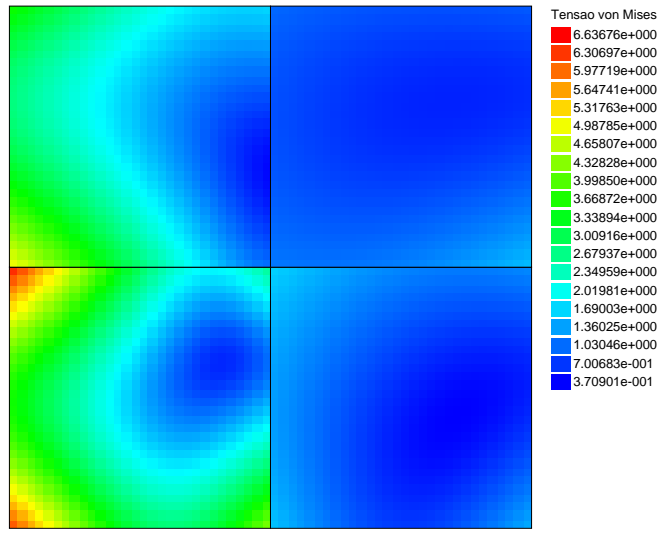

(f) Tensões de von Mises
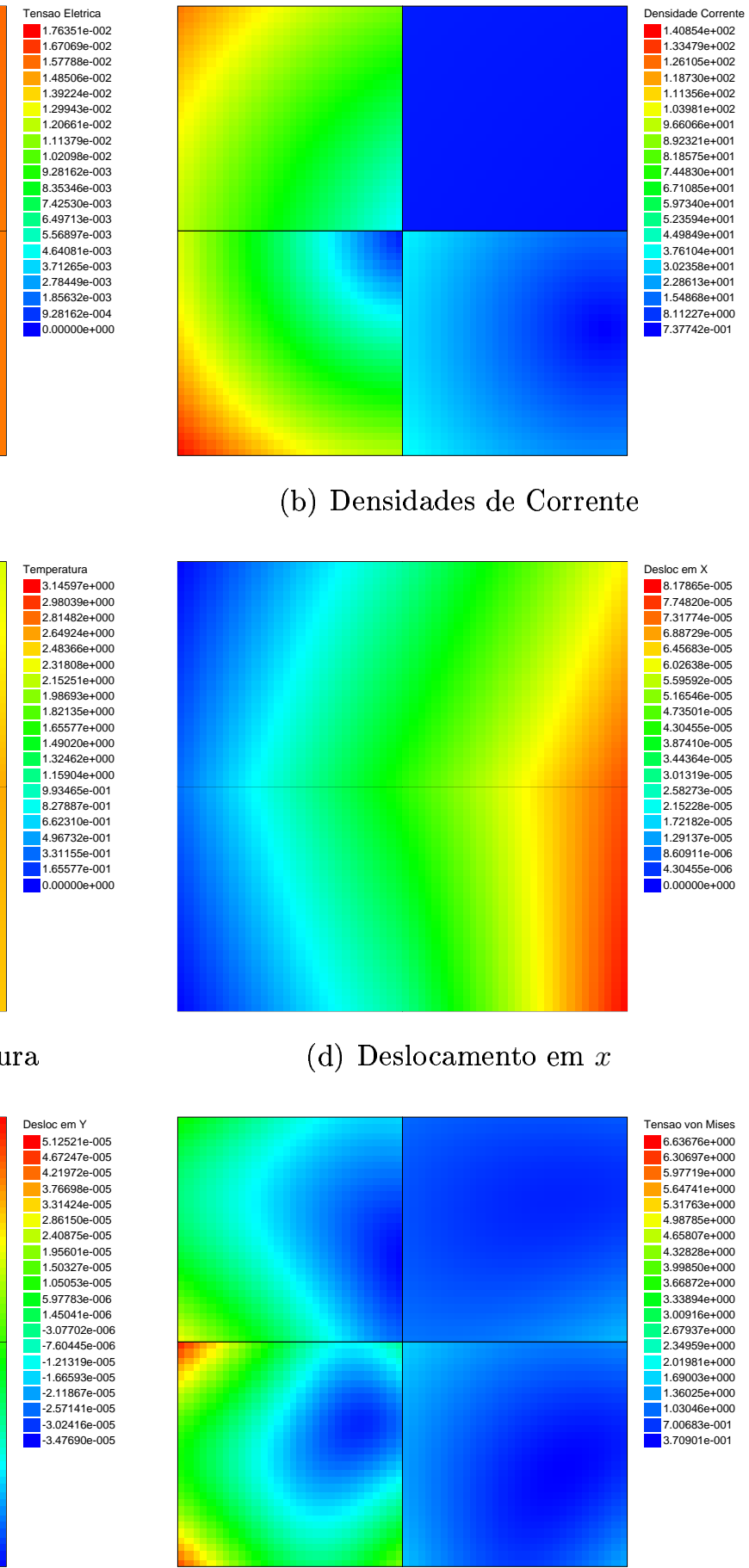
Tabela B.3: Resultados da Análise Elétrica - Densidades de Corrente.

\begin{tabular}{|c|c|c|c|}
\hline Elem & $\begin{array}{c}\text { Nó } \\
\text { Global }\end{array}$ & $\begin{array}{c}\text { Programa em C } \\
\left(\mathrm{A} / \mathrm{mm}^{2}\right)\end{array}$ & $\begin{array}{c}\text { ANSYS } \\
\left(\mathrm{A} / \mathrm{mm}^{2}\right)\end{array}$ \\
\hline \hline \multirow{4}{*}{1} & 1 & 140,8538 & \\
& 2 & 97,13475 & 71,104 \\
& 3 & 3,728823 & \\
\hline \multirow{4}{*}{2} & 4 & 102,0712 & \\
& 2 & 37,19669 & \\
& 3 & 20,12132 & 14,144 \\
& 4 & 15,66511 & \\
& 1 & 94,98745 & \\
& 2 & 34,63073 & \\
& 3 & 86,15804 & 79,803 \\
& 4 & 125,4226 & \\
\hline \multirow{4}{*}{4} & 1 & 3,728795 & \\
& 2 & 2,622763 & \multirow{2}{*}{3,5357} \\
& 3 & 3,575093 & \\
\hline
\end{tabular}

Tabela B.4: Resultados da Análise Eletrotérmica (sem convecção) Temperaturas.

\begin{tabular}{|c|c|c|}
\hline Nó & Programa em C (K) & ANSYS (K) \\
\hline \hline 1 & 0,000000 & 0,0000 \\
2 & 3,145972 & 3,1460 \\
3 & 2,520995 & 2,5210 \\
4 & 2,562642 & 2,5626 \\
5 & 2,147170 & 2,1472 \\
6 & 2,626877 & 2,6269 \\
7 & 0,000000 & 0,0000 \\
8 & 2,014976 & 2,0150 \\
9 & 2,242222 & 2,2422 \\
\hline
\end{tabular}

caso, as tensões de von Mises nodais são as mesmas, observando-se facilmente que o valor máximo de ambos os casos é o mesmo e ocorre no mesmo nó. Os resultados exibidos pelas figuras geradas pelo programa em $\mathrm{C}$ possuem um maior nível de detalhamento que as produzidas pelo ANSYS.

A formulação usada para o cálculo da convecção no ANSYS só é igual ao implementado no programa em C para o elemento SOLID90, dada pela seguinte equação que resulta em uma matriz cheia:

$$
\boldsymbol{h}^{e}=\int_{S^{e}} h_{0} \boldsymbol{N}_{\boldsymbol{f}}^{t} \boldsymbol{N}_{\boldsymbol{f}} d S^{e}
$$

Para os outros elementos, a matriz de conveç̧ão utilizada é mais simples, 
Tabela B.5: Resultados da Análise Termoelástica (sem convecção) Deslocamentos.

\begin{tabular}{|c|c|c|c|}
\hline \multicolumn{2}{|c|}{$\begin{array}{c}\text { Nó e Grau de } \\
\text { Liberdade }\end{array}$} & $\begin{array}{c}\text { Programa em C } \\
\left(10^{-5} \mathrm{~mm}\right)\end{array}$ & $\begin{array}{c}\text { ANSYS } \\
\left(10^{-5} \mathrm{~mm}\right)\end{array}$ \\
\hline \hline \multirow{2}{*}{1} & $x$ & 0,000000 & 0,0000 \\
& $y$ & 0,000000 & 0,0000 \\
\hline \multirow{2}{*}{2} & $x$ & 2,911989 & 2,9120 \\
& $y$ & $-3,475770$ & $-3,4758$ \\
\hline \multirow{2}{*}{3} & $x$ & 8,178652 & 8,1786 \\
& $y$ & $-3,476900$ & $-3,4769$ \\
\hline \multirow{2}{*}{4} & $x$ & 0,8769327 & 0,87693 \\
& $y$ & 0,3715104 & 0,37151 \\
\hline \multirow{2}{*}{5} & $x$ & 4,371106 & 4,3711 \\
& $y$ & 0,5949915 & 0,59499 \\
\hline \multirow{2}{*}{6} & $x$ & 7,599269 & 7,5993 \\
& $y$ & 1,282322 & 1,2823 \\
\hline \multirow{2}{*}{7} & $x$ & 0,000000 & 0,0000 \\
& $y$ & 0,000000 & 0,0000 \\
\hline \multirow{2}{*}{8} & $x$ & 2,781428 & 2,7814 \\
& $y$ & 3,809065 & 3,8091 \\
\hline \multirow{2}{*}{9} & $x$ & 6,269234 & 6,2692 \\
& $y$ & 5,125209 & 5,1252 \\
\hline
\end{tabular}

Tabela B.6: Resultados da Análise Termoelástica (sem convecção) - Tensões Mecânicas (programa em C).

\begin{tabular}{|c|c|c|c|c|c|}
\hline \multirow{3}{*}{ Elem } & \multirow{2}{*}{$\begin{array}{c}\text { Nó } \\
\text { Global }\end{array}$} & \multicolumn{4}{|c|}{$\begin{array}{c}\text { Tensões Mecânicas } \\
\left(\mathrm{N} / \mathrm{mm}^{2}\right)\end{array}$} \\
\cline { 3 - 6 } & & $\sigma_{x}$ & $\sigma_{y}$ & $\tau_{x y}$ & $\tau_{v M}$ \\
\hline \hline \multirow{4}{*}{1} & 1 & 6,296112 & 2,650234 & $-1,864815$ & 6,356980 \\
& 2 & $-4,176204$ & $-2,513233$ & $-1,447064$ & 4,420772 \\
& 5 & 1,116728 & 1,944198 & 1,207360 & 2,688681 \\
& 4 & $-2,966423$ & $-7,447802$ & 0,7896099 & 6,636757 \\
\hline \multirow{4}{*}{2} & 2 & $-0,3303035$ & $-1,334496$ & 0,6121319 & 1,604153 \\
& 3 & 1,573382 & $1,147287 \mathrm{e}$ & $-0,2437268$ & 1,471355 \\
& 6 & $-1,185884$ & 0,09973856 & 0,04532147 & 1,241253 \\
& 5 & $-0,1837701$ & 0,5237554 & 0,9011802 & 1,685443 \\
\hline \multirow{4}{*}{3} & 4 & $-2,628787 \mathrm{e}$ & $-5,874781$ & $-0,2743499$ & 5,119162 \\
& 5 & $-0,1832383$ & $-0,4184366$ & $-0,5735941$ & 1,057839 \\
& 8 & $-0,7028851$ & $-0,3395950$ & 0,9318037 & 1,724949 \\
& 7 & 3,244702 & 0,5971960 & 1,231048 & 3,673343 \\
\hline \multirow{4}{*}{4} & 5 & 0,01109975 & $-0,009120299$ & $-0,6474860$ & 1,121616 \\
& 6 & $-1,544007$ & $-0,6618078$ & $-0,4611773$ & 1,561462 \\
& 9 & 0,5680864 & 1,077668 & $-0,009967588$ & 0,9339060 \\
& 8 & 1,091396 & 0,6985587 & $-0,1962763$ & 1,016021 \\
\hline
\end{tabular}

sendo uma matriz diagonal cujos termos são dados pelo vetor (ANSYS, INC., 2001):

$$
\boldsymbol{h}_{\boldsymbol{A N S Y S}}^{e}=\int_{S^{e}} h_{0} \boldsymbol{N}_{\boldsymbol{f}} d S^{e}
$$


Tabela B.7: Resultados da Análise Termoelástica (sem convecção) - Tensões Mecânicas (ANSYS).

\begin{tabular}{|c|c|c|c|c|}
\hline \multirow{3}{*}{ Elem } & \multirow{2}{*}{ Nó } & \multicolumn{3}{|c|}{ Tensões Mecânicas } \\
\cline { 3 - 5 } & Global $\left./ \mathrm{mm}^{2}\right)$ \\
\cline { 3 - 5 } & & $\sigma_{x}$ & $\sigma_{y}$ & $\tau_{x y}$ \\
\hline \hline \multirow{4}{*}{1} & 1 & 6,2961 & 2,6502 & $-1,8648$ \\
& 2 & $-4,1762$ & $-2,5132$ & $-1,4471$ \\
& 5 & 1,1167 & 1,9442 & 1,2074 \\
& 4 & $-2,9664$ & $-7,4478$ & 0,78961 \\
\hline \multirow{4}{*}{3} & 2 & $-0,33030$ & $-1,3345$ & 0,61213 \\
& 3 & 1,5734 & 1,1473 & $-0,24373$ \\
& 6 & $-1,1859$ & 0,099741 & 0,045322 \\
& 5 & $-0,18377$ & 0,52375 & 0,90118 \\
\hline \multirow{4}{*}{4} & 4 & $-2,6288$ & $-5,8748$ & $-0,27435$ \\
& 5 & $-0,18324$ & $-0,41844$ & $-0,57359$ \\
& 8 & $-0,70288$ & $-0,33959$ & 0,93180 \\
& 7 & 3,2447 & 0,59720 & 1,2310 \\
\hline & 5 & 0,011100 & $-0,0091213$ & $-0,64749$ \\
& 6 & $-1,5440$ & $-0,66181$ & $-0,46118$ \\
& 9 & 0,56808 & 1,0777 & $-0,0099683$ \\
& 8 & 1,0914 & 0,69856 & $-0,19628$ \\
\hline \multirow{3}{*}{} & & \multicolumn{3}{|c}{} \\
\hline
\end{tabular}

onde $h_{0}$ é o coeficiente de convecção e $S^{e}$ é a superfície do elemento onde a conveç̧ão ocorre.

A seguir, os resultados do programa em $\mathrm{C}$ utilizando a matriz de convecção $\boldsymbol{h}^{\boldsymbol{e}}$ e $\boldsymbol{h}_{\boldsymbol{A N S Y S}}^{\boldsymbol{e}}$ e do ANSYS. A conveç̧ão, de coeficiente $h_{0}=1 \times 10^{-3} \mathrm{~W} / \mathrm{mm}^{2} \cdot \mathrm{K}$, foi considerada apenas na face superior (visível), desprezando a convecção nas quatro laterais e na parte inferior. A análise elétrica é essencialmente a mesma e não é reapresentada.

Tabela B.8: Resultados da Análise Eletrotérmica (com convecção) Temperaturas.

\begin{tabular}{|c|c|c|c|}
\hline \multirow{2}{*}{ Nó } & \multicolumn{2}{|c|}{ Programa em C (K) } & \multirow{2}{*}{ ANSYS (K) } \\
\cline { 2 - 3 } & $\boldsymbol{h}^{\boldsymbol{e}}$ & $\boldsymbol{h}_{\boldsymbol{A N S Y} \boldsymbol{S}}$ & \\
\hline \hline 1 & 0,000000 & 0,000000 & 0,0000 \\
2 & 1,941484 & 1,852670 & 1,8527 \\
3 & 1,345727 & 1,303472 & 1,3035 \\
4 & 1,744092 & 1,661494 & 1,6615 \\
5 & 1,201885 & 1,170405 & 1,1704 \\
6 & 1,387816 & 1,328671 & 1,3287 \\
7 & 0,000000 & 0,000000 & 0,0000 \\
8 & 1,162485 & 1,110737 & 1,1107 \\
9 & 0,9386804 & 0,9432398 & 0,94324 \\
\hline
\end{tabular}

Considerando a matriz de convecção baseada em $\boldsymbol{h}_{A N S Y S}^{e}$, as diferenças entre os resultados do programa em C e do ANSYS também são da ordem de 0,002\%. 


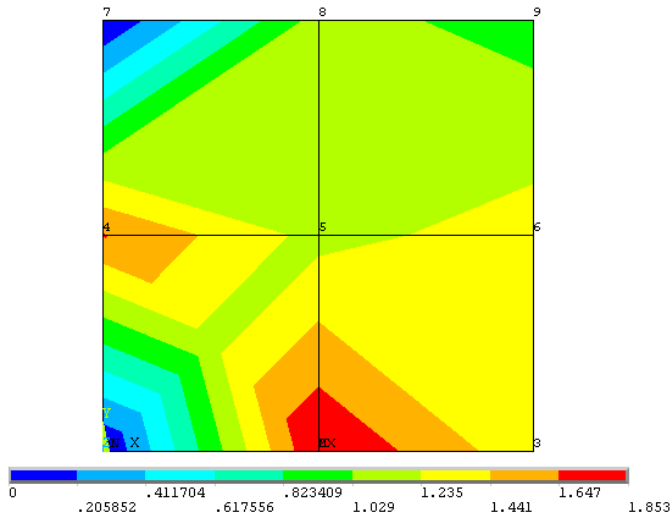

(a) Diferenças de Temperatura

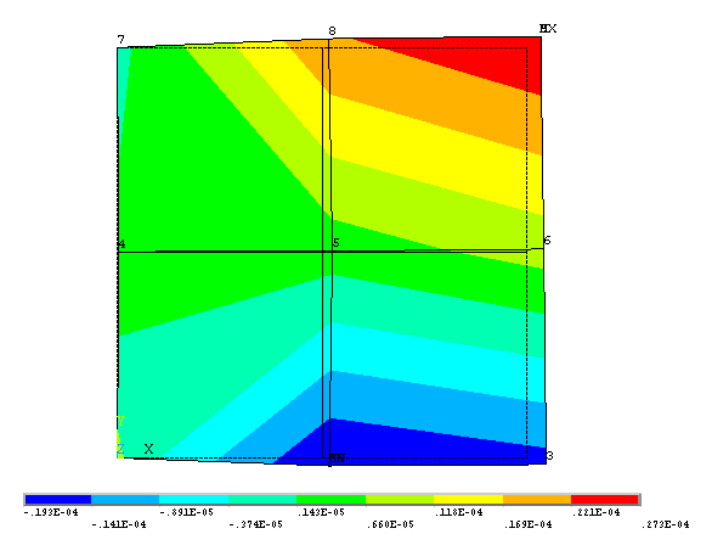

(c) Deslocamento em $y$

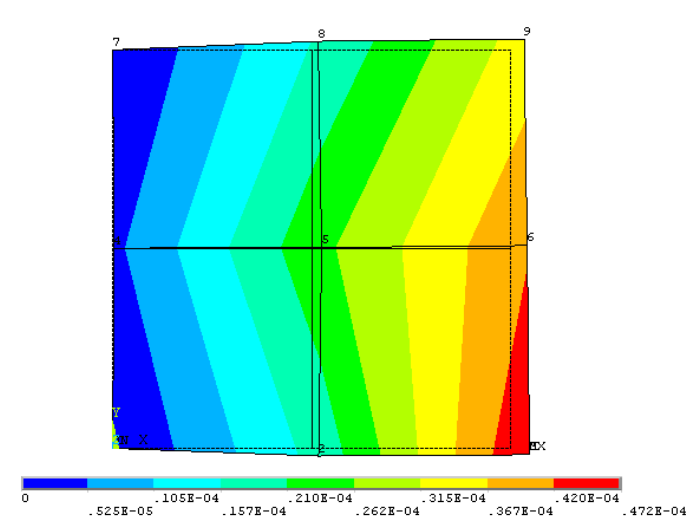

(b) Deslocamento em $x$

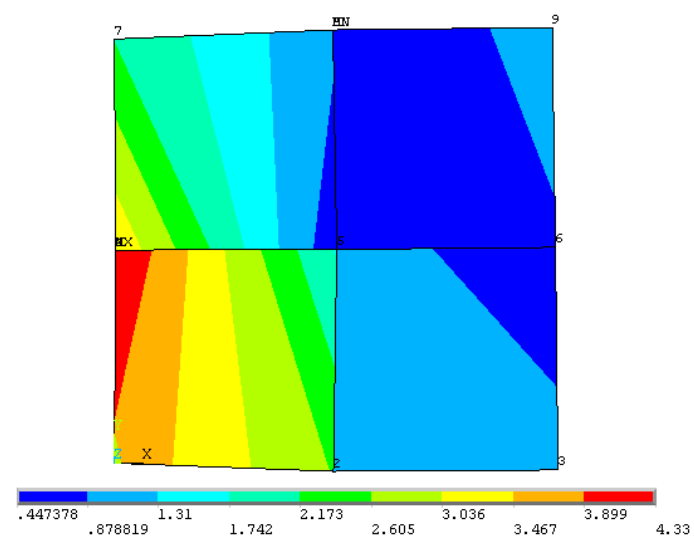

(d) Tensões de von Mises

Figura B.4: Resultados das Análises por ANSYS (com convecção). 


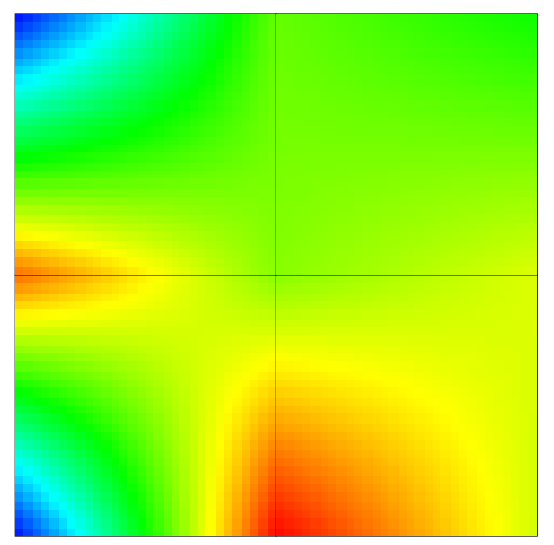

(a) Diferenças de Temperatura

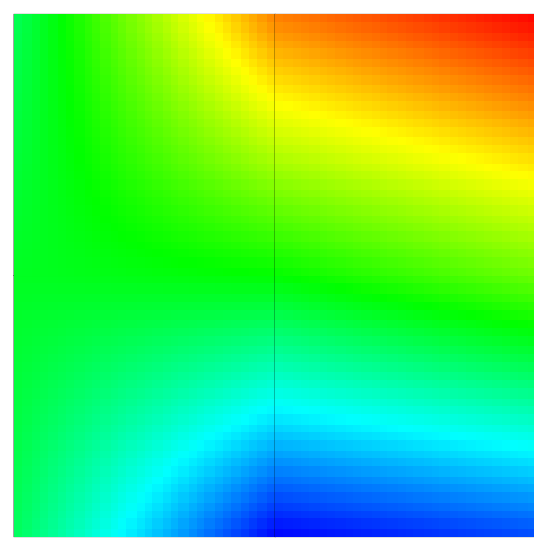

(c) Deslocamento em $y$

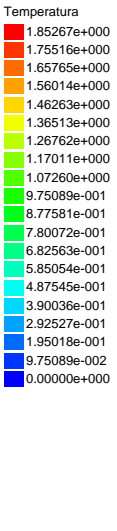

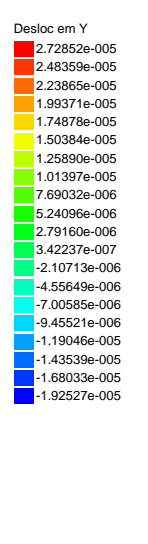
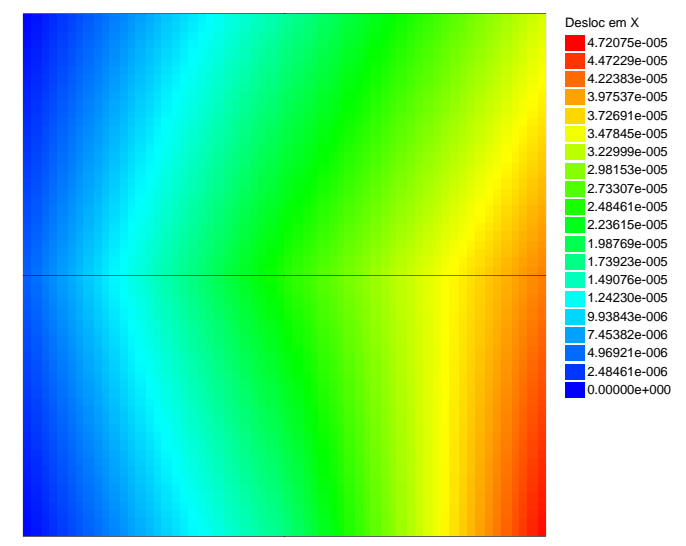

(b) Deslocamento em $x$
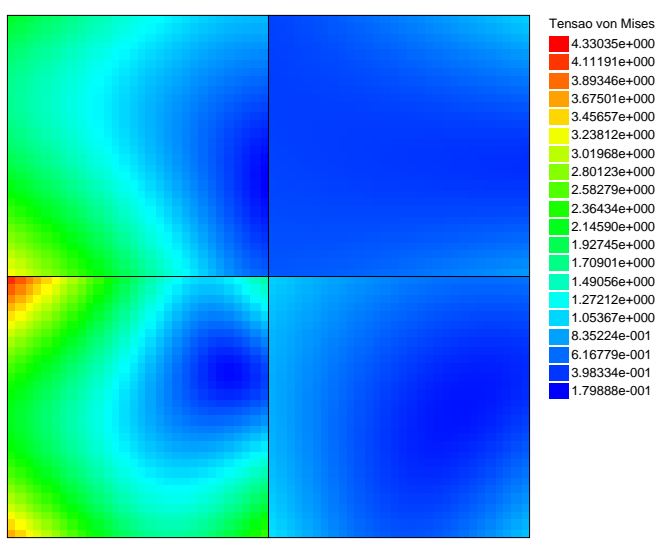

(d) Tensões de von Mises

Figura B.5: Resultados das Análises pelo programa em C (com convecção matriz $\left.\boldsymbol{h}_{A N S Y S}^{e}\right)$. 


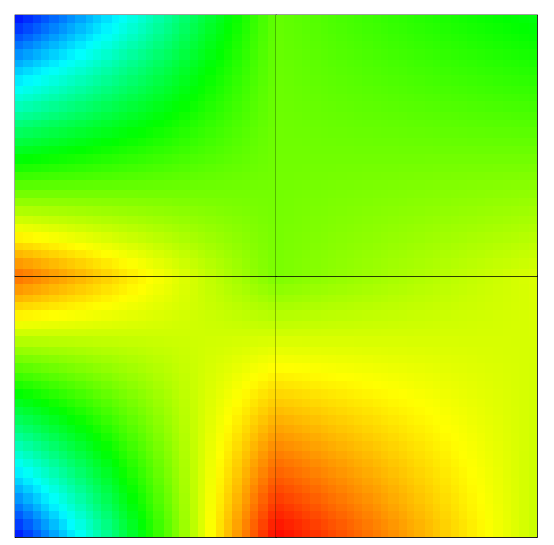

(a) Diferenças de Temperatura

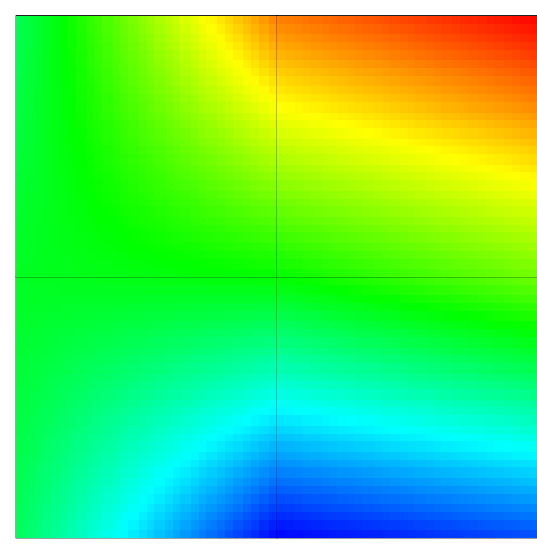

(c) Deslocamento em $y$

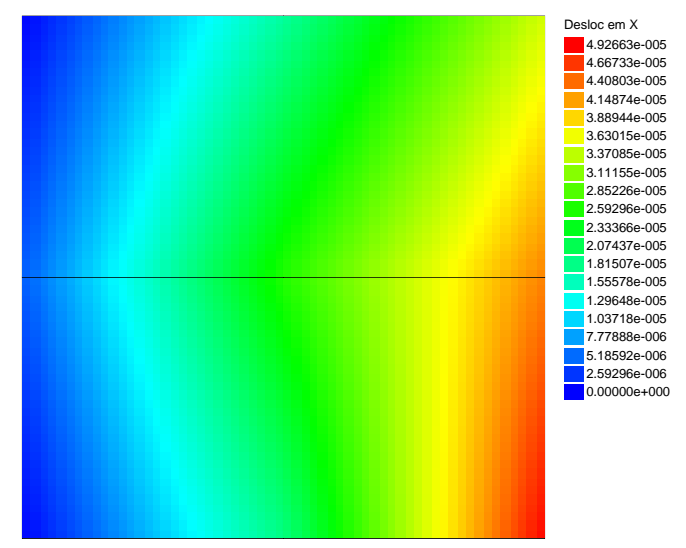

(b) Deslocamento em $x$

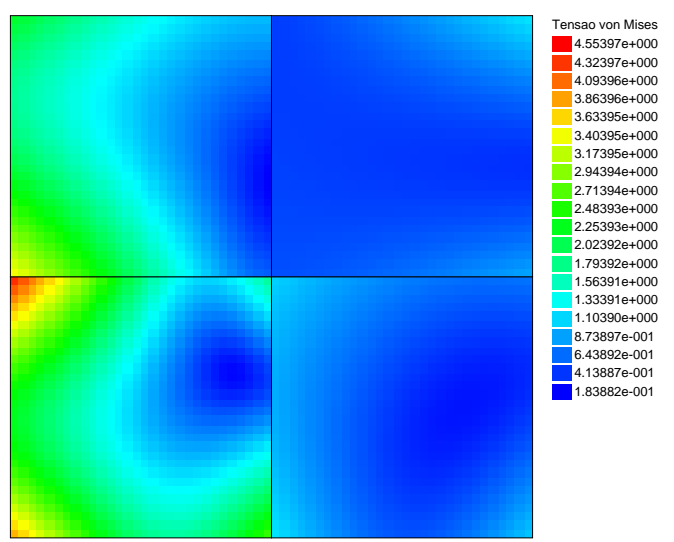

(d) Tensões de von Mises

Figura B.6: Resultados das Análises pelo programa em C (com convecção matriz $\left.\boldsymbol{h}^{e}\right)$. 
Tabela B.9: Resultados da Análise Termoelástica (com convecção) -

Deslocamentos.

\begin{tabular}{|c|c|c|c|c|}
\hline \multirow{2}{*}{\multicolumn{2}{|c|}{$\begin{array}{c}\text { Nó e Grau de } \\
\text { Liberdade }\end{array}$}} & \multicolumn{2}{|c|}{ Programa em C } & \multirow[b]{2}{*}{$\begin{array}{c}\text { ANSYS } \\
\left(10^{-5} \mathrm{~mm}\right)\end{array}$} \\
\hline & & \multirow{3}{*}{$\begin{array}{c}\boldsymbol{h}^{\boldsymbol{e}} \\
\left(10^{-5} \mathrm{~mm}\right) \\
0,000000\end{array}$} & \multirow{2}{*}{$\begin{array}{c}\begin{array}{c}\boldsymbol{h}_{\boldsymbol{A N S Y S}}^{\boldsymbol{e}} \\
\left(10^{-5} \mathrm{~mm}\right)\end{array} \\
0,000000\end{array}$} & \\
\hline \multirow[b]{2}{*}{1} & $\bar{x}$ & & & 0,0000 \\
\hline & $y$ & & 0,000000 & 0,0000 \\
\hline \multirow{2}{*}{2} & $x$ & 1,831741 & 1,749127 & 1,7491 \\
\hline & $y$ & $-1,999457$ & $-1,925266$ & $-1,9253$ \\
\hline \multirow{2}{*}{3} & $x$ & 4,926626 & 4,720754 & 4,7208 \\
\hline & $y$ & $-1,643450$ & $-1,600355$ & $-1,6004$ \\
\hline \multirow[b]{2}{*}{4} & $x$ & 0,4384044 & 0,4189294 & 0,41893 \\
\hline & $y$ & 0,2585099 & 0,2460825 & 0,24608 \\
\hline \multirow[b]{2}{*}{5} & $x$ & 2,618703 & 2,513849 & 2,5138 \\
\hline & $y$ & 0,4175066 & 0,3977298 & 0,39773 \\
\hline \multirow{2}{*}{6} & $x$ & 4,306270 & 4,144527 & 4,1445 \\
\hline & $y$ & 0,9461456 & 0,8926313 & 0,89263 \\
\hline \multirow[b]{2}{*}{7} & $x$ & 0,000000 & 0,000000 & 0,0000 \\
\hline & $y$ & 0,000000 & 0,000000 & 0,0000 \\
\hline \multirow[b]{2}{*}{8} & $x$ & 1,706763 & 1,632193 & 1,6322 \\
\hline & $y$ & 2,242599 & 2,157245 & 2,1572 \\
\hline \multirow[b]{2}{*}{9} & $x$ & 3,488617 & 3,370037 & 3,3700 \\
\hline & $y$ & 2,828459 & 2,728522 & 2,7285 \\
\hline
\end{tabular}

Tabela B.10: Resultados da Análise Termoelástica (com convecção) - Tensões Mecânicas (programa em C $-\boldsymbol{h}^{e}$ ).

\begin{tabular}{|c|c|c|c|c|c|}
\hline \multirow[t]{2}{*}{ Elem } & \multirow{2}{*}{$\begin{array}{c}\text { Nó } \\
\text { Global }\end{array}$} & \multicolumn{4}{|c|}{$\begin{array}{c}\text { Tensões Mecânicas } \\
\left(\mathrm{N} / \mathrm{mm}^{2}\right)\end{array}$} \\
\hline & & $\sigma_{x}$ & $\sigma_{y}$ & $\tau_{x y}$ & $\tau_{v M}$ \\
\hline \multirow{4}{*}{1} & $\bar{~} 1$ & 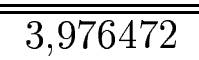 & $\overline{10,718705}$ & "-1,120145 & 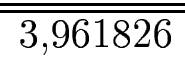 \\
\hline & 2 & $-2,566601$ & $-1,726740$ & $-0,8700346$ & 2,721782 \\
\hline & 5 & 1,181064 & 1,520706 & 0,6787795 & 1,814837 \\
\hline & 4 & $-2,426600$ & $-5,184587$ & 0,4286695 & 4,553974 \\
\hline \multirow{4}{*}{2} & 2 & $-0,4288210$ & $-0,9980680$ & 0,4798725 & 1,201194 \\
\hline & 3 & 1,203405 & 0,7791162 & $-0,1109862$ & 1,074508 \\
\hline & 6 & $-0,6199413$ & 0,1374873 & $-0,03850714$ & 0,7020772 \\
\hline & 5 & $-0,1959880$ & 0,4164824 & 0,5523516 & 1,099441 \\
\hline \multirow{4}{*}{3} & $\overline{4}$ & $\begin{array}{l}-2,031932 \\
\end{array}$ & $-4,079787$ & $-0,1173086$ & 3,539046 \\
\hline & 5 & 0,1803552 & $-0,1179095$ & $-0,3161212$ & 0,6062130 \\
\hline & 8 & $-0,2922742$ & $-0,1929136$ & 0,5586736 & 1,001302 \\
\hline & 7 & $1,979519 \mathrm{e}$ & 0,3292901 & 0,7574863 & 2,257532 \\
\hline \multirow{4}{*}{4} & 5 & $-0,2253720$ & $-0,02800731$ & $-0,2750404$ & 0,5217342 \\
\hline & 6 & $-0,9483700$ & $-0,6688866$ & $-0,2073841$ & 0,9173263 \\
\hline & 9 & 1,083333 & 1,227504 & $-0,1663247$ & 1,197319 \\
\hline & 8 & 0,1317594 & 0,1938114 & $-0,2339811$ & 0,4400322 \\
\hline
\end{tabular}

As tensões de von Mises, ao utilizar $\boldsymbol{h}_{\boldsymbol{A N S Y} \boldsymbol{S}}^{e}$, também podem ser verificadas comparando-se as figuras B.4(d) e B.5(d), observando-se também que o valor 
Tabela B.11: Resultados da Análise Termoelástica (com convecção) - Tensões Mecânicas (programa em C - $\boldsymbol{h}_{\boldsymbol{A N S Y S}}^{e}$ ).

\begin{tabular}{|c|c|c|c|c|c|}
\hline \multirow[t]{2}{*}{ Elem } & \multirow{2}{*}{$\begin{array}{c}\text { Nó } \\
\text { Global }\end{array}$} & \multicolumn{4}{|c|}{$\begin{array}{c}\text { Tensões Mecânicas } \\
\left(\mathrm{N} / \mathrm{mm}^{2}\right)\end{array}$} \\
\hline & & $\sigma_{x}$ & $\sigma_{y}$ & $\tau_{x y}$ & $\tau_{v M}$ \\
\hline \multirow[b]{2}{*}{1} & $\begin{array}{l}1 \\
2\end{array}$ & $\begin{array}{c}3,796632 \\
-2,436036\end{array}$ & $\begin{array}{c}1,639591 \\
-1,612468\end{array}$ & $\begin{array}{c}-1,080883 \\
-0,8327568\end{array}$ & $\begin{array}{l}3,792449 \\
2,585874\end{array}$ \\
\hline & $\begin{array}{l}5 \\
4\end{array}$ & $\begin{array}{c}1,071557 \\
-2,274616\end{array}$ & $\begin{array}{r}1,398873 \\
-4,927909\end{array}$ & $\begin{array}{l}0,6575474 \\
0,4094214\end{array}$ & $\begin{array}{l}1,703882 \\
4,330351\end{array}$ \\
\hline \multirow[b]{2}{*}{2} & $\begin{array}{l}2 \\
3\end{array}$ & $\begin{array}{c}-0,3806500 \\
1,128109\end{array}$ & $\begin{array}{c}-0,9253022 \\
0,7261971\end{array}$ & $\begin{array}{c}0,4574795 \\
-0,1055144\end{array}$ & $\begin{array}{l}1,129922 \\
1,007056\end{array}$ \\
\hline & $\begin{array}{l}6 \\
5\end{array}$ & $\begin{array}{l}-0,5700415 \\
-0,2178627\end{array}$ & $\begin{array}{l}0,1540342 \\
0,3634729\end{array}$ & $\begin{array}{c}-0,03414429 \\
0,5288496\end{array}$ & $\begin{array}{c}0,6633078 \\
1,047764\end{array}$ \\
\hline \multirow[b]{2}{*}{3} & $\begin{array}{l}4 \\
5\end{array}$ & $\begin{array}{l}-1,913877 \\
0,1345202\end{array}$ & $\begin{array}{c}-3,879604 \\
-0,1471171\end{array}$ & $\begin{array}{l}-0,1122177 \\
-0,3064922\end{array}$ & $\begin{array}{c}3,365553 \\
0,5842446\end{array}$ \\
\hline & $\begin{array}{l}8 \\
7\end{array}$ & $\begin{array}{c}-0,2716416 \\
1,893460\end{array}$ & $\begin{array}{c}-0,1647298 \\
0,3162817\end{array}$ & $\begin{array}{l}0,5355526 \\
0,7298272\end{array}$ & $\begin{array}{c}0,9574066 \\
2,164324\end{array}$ \\
\hline \multirow[b]{2}{*}{$\mathbf{t}$} & $\begin{array}{l}5 \\
6\end{array}$ & $\begin{array}{l}-0,2573183 \\
-0,8549006\end{array}$ & $\begin{array}{c}-0,07242221 \\
-0,5603969\end{array}$ & $\begin{array}{l}-0,2775185 \\
-0,2006208\end{array}$ & $\begin{array}{l}0,5327961 \\
0,8285905\end{array}$ \\
\hline & $\begin{array}{l}9 \\
8\end{array}$ & $\begin{array}{l}0,9432319 \\
0,2094342\end{array}$ & $\begin{array}{c}1,083940 \\
0,2405348\end{array}$ & $\begin{array}{l}-0,1458170 \\
-0,2227147\end{array}$ & $\begin{array}{c}1,051662 \\
0,4473801\end{array}$ \\
\hline
\end{tabular}

Tabela B.12: Resultados da Análise Termoelástica (com convecção) - Tensões Mecânicas (ANSYS).

\begin{tabular}{|c|c|c|c|c|}
\hline \multirow{3}{*}{ Elem } & \multirow{2}{*}{ Nó } & \multicolumn{3}{|c|}{ Tensões Mecânicas } \\
\cline { 3 - 5 } & Global $\left./ \mathrm{mm}^{2}\right)$ \\
\cline { 3 - 5 } & & $\sigma_{x}$ & $\sigma_{y}$ & $\tau_{x y}$ \\
\hline \hline \multirow{4}{*}{1} & 1 & 3,7966 & 1,6396 & $-1,0809$ \\
& 2 & $-2,4360$ & $-1,6125$ & $-0,83276$ \\
& 5 & 1,0716 & 1,3989 & 0,65755 \\
& 4 & $-2,2746$ & $-4,9279$ & 0,40942 \\
\hline \multirow{4}{*}{3} & 2 & $-0,38065$ & $-0,92530$ & 0,45748 \\
& 3 & 1,1281 & 0,72620 & $-0,10551$ \\
& 6 & $-0,57004$ & 0,15404 & $-0,034144$ \\
& 5 & $-0,21786$ & 0,36347 & 0,52885 \\
\hline \multirow{4}{*}{4} & 4 & $-1,9139$ & $-3,8796$ & $-0,11222$ \\
& 5 & 0,13452 & $-0,14712$ & $-0,30649$ \\
& 8 & $-0,27164$ & $-0,16473$ & 0,53555 \\
& 7 & 1,8935 & 0,31628 & 0,72983 \\
\hline & 5 & $-0,25732$ & $-0,072423$ & $-0,27752$ \\
& 6 & $-0,85490$ & $-0,56039$ & $-0,20062$ \\
& 9 & 0,94323 & 1,0839 & $-0,14582$ \\
& 8 & 0,20943 & 0,24053 & $-0,22271$ \\
\hline
\end{tabular}

máximo de ambos os casos é o mesmo e ocorre no mesmo nó. Já utilizando a matriz $\boldsymbol{h}^{e}$, as temperaturas são maiores, o que acarreta deslocamentos e tensões mecânicas mais altas. 


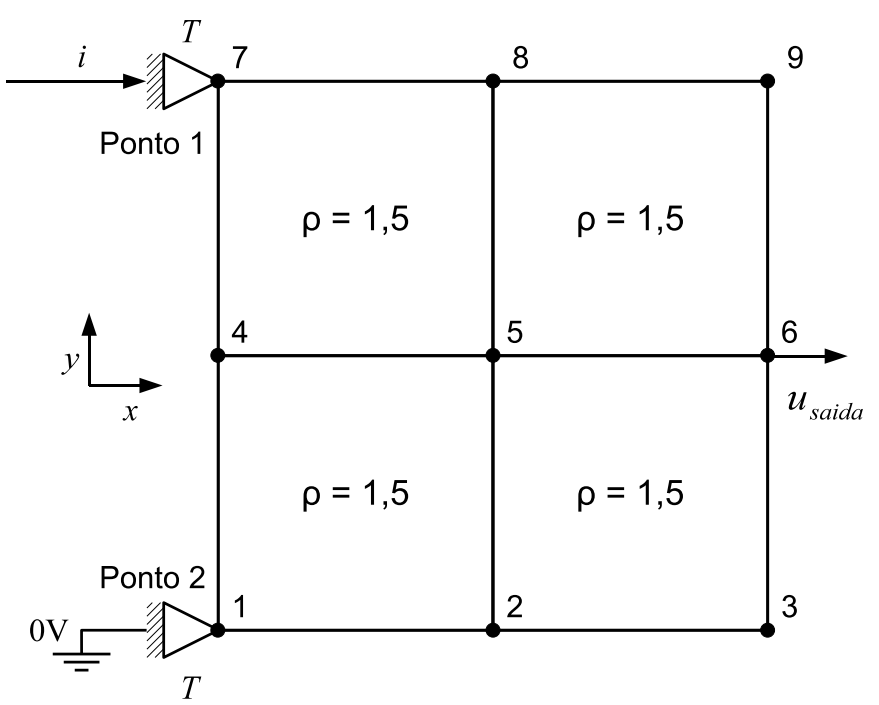

Figura B.7: Modelo para verificação das sensibilidades - com indicação dos nós, pseudo-densidades, condições de contorno e deslocamento de saída.

\section{B.2 Verificação das Sensibilidades}

Para verificar as sensibilidades, o mesmo modelo com quatro elementos e dois materiais foi utilizado. A pseudo-densidade para os quatro elementos foi considerada uniforme e igual a 1,5. O desvio padrão utilizado para a função de pico foi de 0,3. A figura B.7 ilustra o modelo, com a identificação dos nós, pseudo-densidades, condições de contorno e o deslocamento de saída.

Calculando as sensibilidades $\frac{d u_{\text {saida }}}{d \rho^{e}}$ do deslocamento de saída do modelo utilizado em relação às variáveis de projeto (pseudo-densidades $\rho^{e}$ de cada elemento $e$ ), utilizando o método adjunto conforme descrito no relatório anterior, comparam-se estes valores com as sensibilidades $\frac{\Delta u_{\text {saida }}}{\Delta \rho^{e}}$ obtidas pelo Método de Diferenças Finitas (MDF). A sensibilidade por MDF tende à sensibilidade calculada pelo método adjunto quando $\Delta \rho^{e}$ se aproxima de zero. Para sensibilidades analíticas relativamente baixas, o $\Delta \rho^{e}$ deve ser muito menor para que a aproximação seja satisfatória. Deve-se notar que, devido à precisão numérica do computador ser finita, a redução de um $\Delta \rho^{e}$ muito pequeno pode não resultar em uma sensibilidade por MDF mais próxima da analítica, caso esta já seja naturalmente baixa. Assim:

$$
\frac{d u_{\text {saida }}}{d \rho^{e}} \approx \frac{\Delta u_{\text {saida }}}{\Delta \rho^{e}}=\frac{u_{\text {saida }}\left(\boldsymbol{\rho}_{\text {mod }}\right)-u_{\text {saida }}(\boldsymbol{\rho})}{\Delta \rho^{e} \rightarrow 0}
$$

onde $\rho$ é o vetor de variáveis de projeto em torno do qual deseja-se calcular a sensibilidade; $\boldsymbol{\rho}_{\text {mod }}$ é o vetor de variáveis de projeto cuja pseudo-densidade de um dado elemento $e$ foi acrescida de $\Delta \rho^{e}$. 
Note que, para cada cálculo de $u_{\text {saida }}$, uma análise pelo MEF é necessária. Dado o alto custo computacional, esse método de cálculo das sensibilidades não é factível para implementação em uma rotina de otimização, mas é útil para verificação da implementação de um método analítico.

Foram calculadas as sensibilidades pelo MDF, para diversos $\Delta \rho^{e}$, e pelo método adjunto. Os resultados são mostrados na tabela B.13, onde pode-se ver a relativa dificuldade em se aproximar a sensibilidade calculada pelo MDF de uma analítica de valor reduzido.

Tabela B.13: Cálculo das Sensibilidades pelo método adjunto e Método de Diferenças Finitas.

\begin{tabular}{|c|c|c|c|c|c|}
\hline \multirow[b]{2}{*}{$\Delta \rho^{e}$} & \multirow{2}{*}{ Elem } & \multirow[b]{2}{*}{$\Delta u_{\text {saida }}$} & \multicolumn{2}{|c|}{ Sensibilidade } & \multirow{2}{*}{ Diferença } \\
\hline & & & Adjunto & MDF & \\
\hline \multirow{4}{*}{$10^{-2}$} & $\overline{1} 1$ & $-1,019$ & $-1,183 \times 10^{2}$ & $-1,019 \times 10^{2}$ & $\overline{13,87 \%}$ \\
\hline & 2 & $1,271 \times 10^{-1}$ & $3,346 \times 10^{-1}$ & $1,271 \times 10^{1}$ & $-3.700 \%$ \\
\hline & 3 & $-1,019$ & $-1,183 \times 10^{2}$ & $-1,019 \times 10^{2}$ & $13,87 \%$ \\
\hline & 4 & $1,271 \times 10^{-1}$ & $3,346 \times 10^{-1}$ & $1,271 \times 10^{1}$ & $-3.700 \%$ \\
\hline \multirow{4}{*}{$10^{-3}$} & 1 & $-1,169 \times 10^{-1}$ & $-1,183 \times 10^{2}$ & $-1,169 \times 10^{2}$ & $1,212 \%$ \\
\hline & 2 & $1,548 \times 10^{-3}$ & $3,346 \times 10^{-1}$ & 1,548 & $-362,7 \%$ \\
\hline & 3 & $-1,169 \times 10^{-1}$ & $-1,183 \times 10^{2}$ & $-1,169 \times 10^{2}$ & $1,212 \%$ \\
\hline & 4 & $1,548 \times 10^{-3}$ & $3,346 \times 10^{-1}$ & 1,548 & $-362,7 \%$ \\
\hline \multirow{4}{*}{$10^{-4}$} & 1 & $-1,182 \times 10^{-2}$ & $-1,183 \times 10^{2}$ & $-1,182 \times 10^{2}$ & $0,1162 \%$ \\
\hline & 2 & $4,575 \times 10^{-5}$ & $3,346 \times 10^{-1}$ & $4,575 \times 10^{-1}$ & $-36,74 \%$ \\
\hline & 3 & $-1,182 \times 10^{-2}$ & $-1,183 \times 10^{2}$ & $-1,182 \times 10^{2}$ & $0,1162 \%$ \\
\hline & 4 & $4,575 \times 10^{-5}$ & $3,346 \times 10^{-1}$ & $4,575 \times 10^{-1}$ & $-36,74 \%$ \\
\hline \multirow{4}{*}{$10^{-5}$} & 1 & $-1,183 \times 10^{-3}$ & $-1,183 \times 10^{2}$ & $-1,183 \times 10^{2}$ & $0,03922 \%$ \\
\hline & 2 & $3,518 \times 10^{-6}$ & $3,346 \times 10^{-1}$ & $3,518 \times 10^{-1}$ & $-5,150 \%$ \\
\hline & 3 & $-1,183 \times 10^{-3}$ & $-1,183 \times 10^{2}$ & $-1,183 \times 10^{2}$ & $0,03922 \%$ \\
\hline & 4 & $3,518 \times 10^{-6}$ & $3,346 \times 10^{-1}$ & $3,518 \times 10^{-1}$ & $-5,150 \%$ \\
\hline \multirow{4}{*}{$10^{-6}$} & 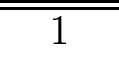 & $-1,183 \times 10^{-4}$ & $-1,183 \times 10^{2}$ & $-1,183 \times 10^{2}$ & 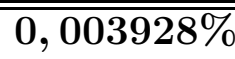 \\
\hline & 2 & $3,363 \times 10^{-7}$ & $3,346 \times 10^{-1}$ & $3,363 \times 10^{-1}$ & $-0,5153 \%$ \\
\hline & 3 & $-1,183 \times 10^{-4}$ & $-1,183 \times 10^{2}$ & $-1,183 \times 10^{2}$ & $0,003928 \%$ \\
\hline & 4 & $3,363 \times 10^{-7}$ & $3,346 \times 10^{-1}$ & $3,363 \times 10^{-1}$ & $-0,5153 \%$ \\
\hline \multirow{4}{*}{$10^{-7}$} & $\overline{\overline{1}}$ & $-1,183 \times 10^{-5}$ & $-1,183 \times 10^{2}$ & $-1,183 \times 10^{2}$ & $0,008034 \%$ \\
\hline & 2 & $5,848 \times 10^{-8}$ & $3,346 \times 10^{-1}$ & $5,848 \times 10^{-1}$ & $-74,77 \%$ \\
\hline & 3 & $-1,183 \times 10^{-5}$ & $-1,183 \times 10^{2}$ & $-1,183 \times 10^{2}$ & $0,008034 \%$ \\
\hline & 4 & $5,848 \times 10^{-8}$ & $3,346 \times 10^{-1}$ & $5,848 \times 10^{-1}$ & $-74,77 \%$ \\
\hline
\end{tabular}

Pelos resultados, foi verificado que, mesmo nos casos de sensibilidade analítica mais baixa, esta pode ser muito bem aproximada por cálculos utilizando o MDF, atingindo diferenças de aproximadamente $0,5 \%$ no melhor caso $\left(\Delta \rho^{e}=10^{-6}\right.$, destacado na tabela B.13). Assim, concluiu-se que o cálculo das sensibilidades pelo método adjunto está correto. 


\section{Apêndice C - Atividades Realizadas no Laboratório Nacional de Luz Síncrotron (LNLS)}

Em paralelo aos trabalhos, durante mais de duas semanas foram realizadas atividades no Laboratório Nacional de Luz Síncrotron (LNLS) visando uma possível fabricação de MEMS com 2 materiais. Um pôster, com resultados e conclusões destas últimas atividades, foi apresentado na $16^{\mathrm{a}}$ Reunião Anual de Usuário do LNLS.

As atividades realizadas no LNLS visaram a fabricação de MEMS eletrotermomecânicos compostos por níquel e cobre. Foi usado o processo de fotolitografia para dar forma ao níquel e cobre a serem eletrodepositados. Na fotolitografia, uma camada de resiste fotosensível (fotoresiste) é depositada, sensibilizada por radiação ultravioleta e revelada quimicamente para servir de forma ao metal eletrodepositado. A sensibilização por ultravioleta é feita sobre certos trechos do fotoresiste, através do uso de máscaras para proteger as partes desejadas. Dependendo do tipo do fotoresiste, a parte sensibilizada é mantida (fotoresiste positivo) ou removida (fotoresiste negativo) durante a revelação química. Assim, uma primeira camada de fotoresiste (AZ4620 - fotoresiste positivo) é depositada para formar a camada de sacrifício que, ao ser removida ao final do processo, permitirá a existência de porções do mecanismo não ancoradas ao substrato de alumina. Uma camada de titânio e ouro é depositada sobre o substrato e a camada de sacrifício pelo processo de "sputtering". Essa camada, por ser condutiva, permite a eletrodeposição de níquel e cobre, e por isso é chamada de "seed layer". Uma segunda e terceira camada de fotoresiste (SU8 - fotoresiste negativo) são depositadas, sensibilizadas e removidas para a eletrodeposição das partes do mecanismo em níquel e em cobre, respectivamente. Um diagrama com as etapas pode ser visto na figura C.1. O mecanismo fabricado é um microposicionador $x y$ que pode ser visto na figura C.2. Um dos mecanismos obtidos pode ser visto na figura C.3. 

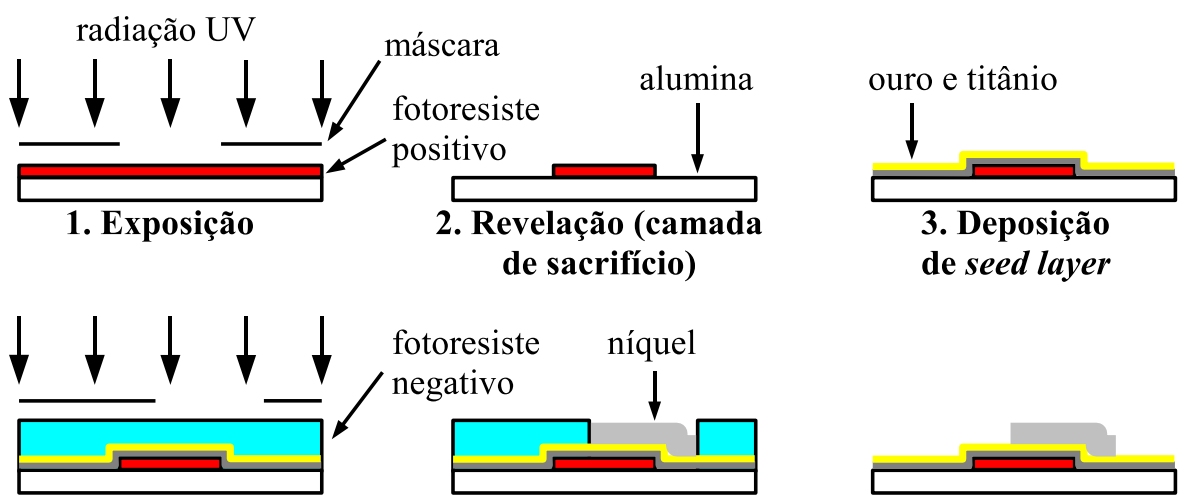

4. Exposição

5. Revelação e Eletrodeposição
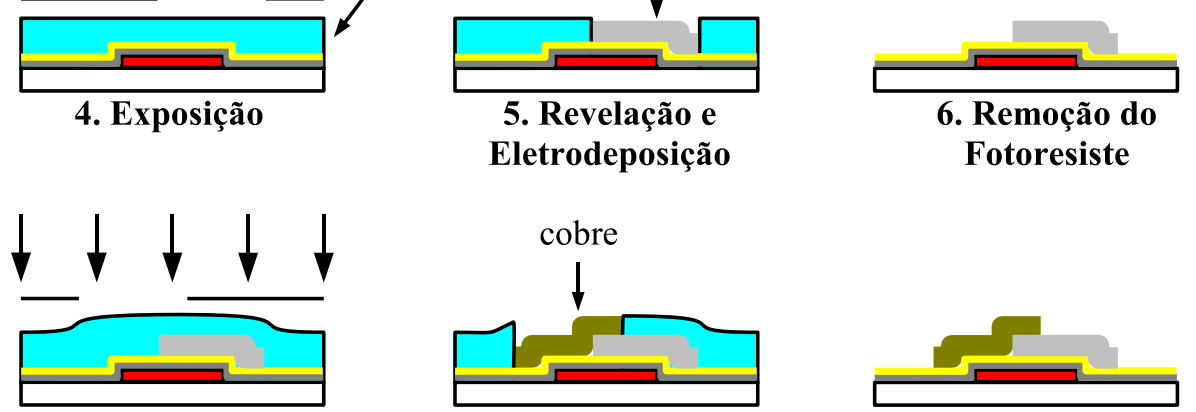

8. Revelação e Eletrodeposição
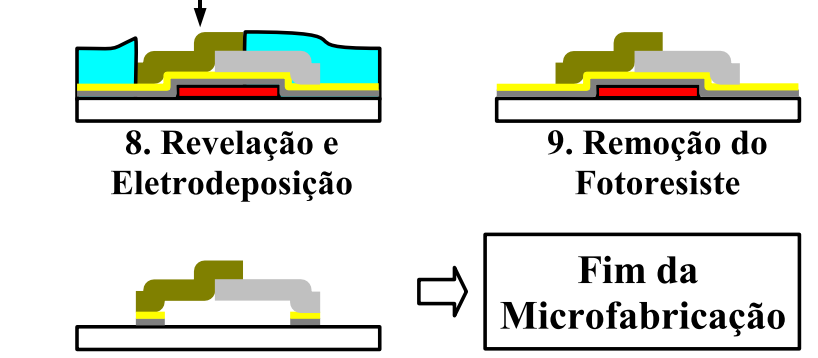

10. Remoção do seed layer e da camada de sacrifício

Figura C.1: Etapas da Microfabricação de um Mecanismo Bimetálico.

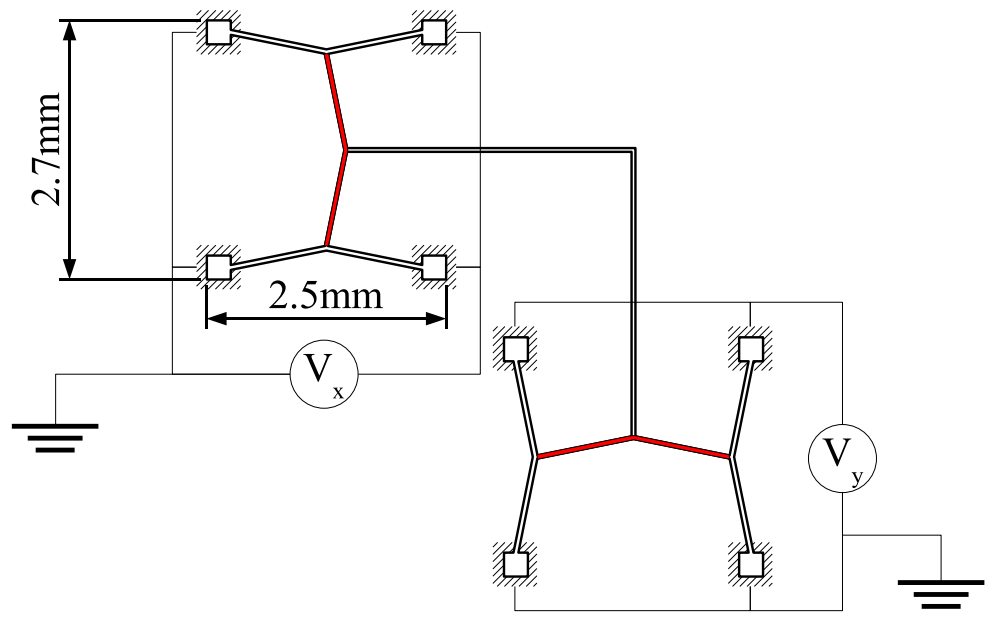

Figura C.2: Microposicionador $x y$ Bimetálico - Esquema (branco - níquel; vermelho - cobre). 


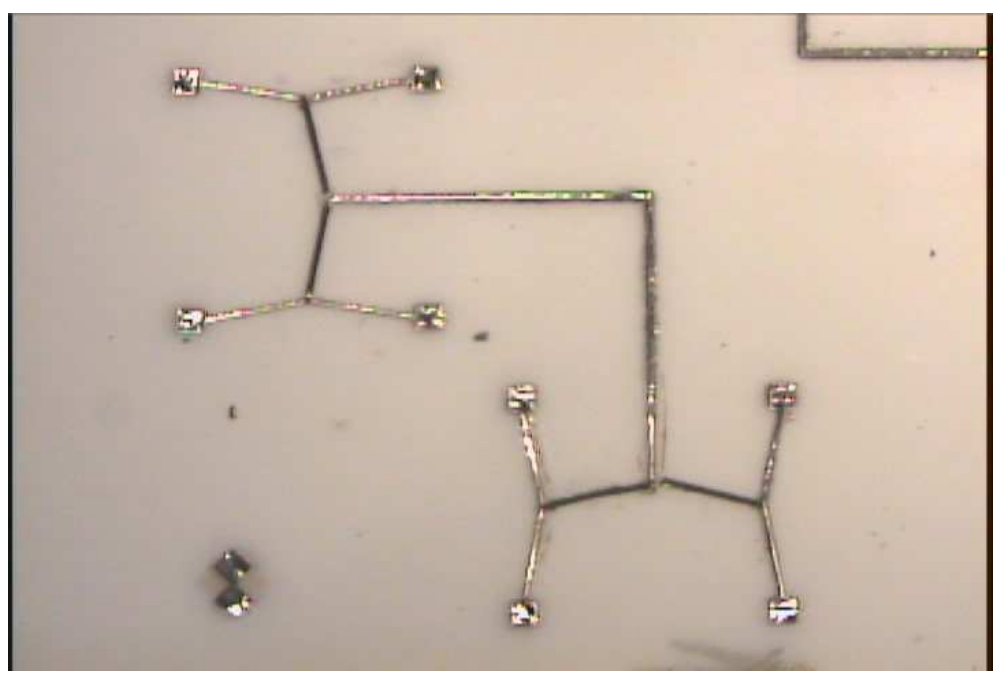

Figura C.3: Microposicionador $x y$ Bimetálico - Protótipo.

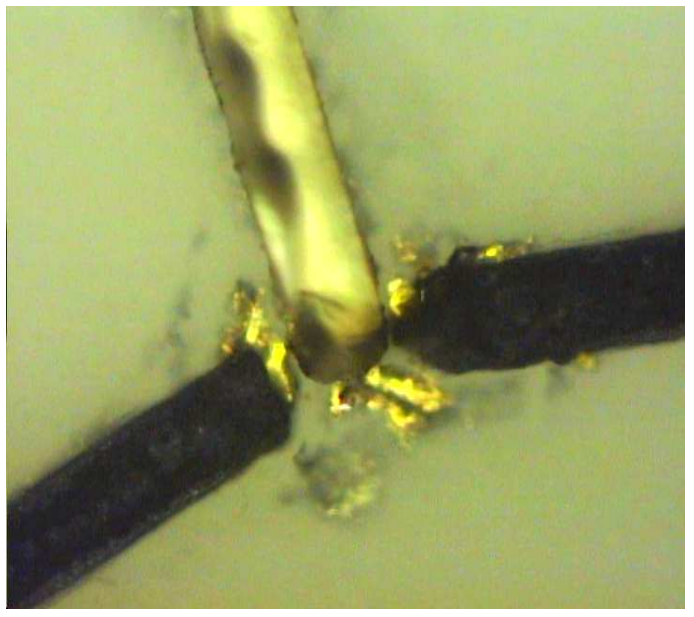

(a) Com problemas

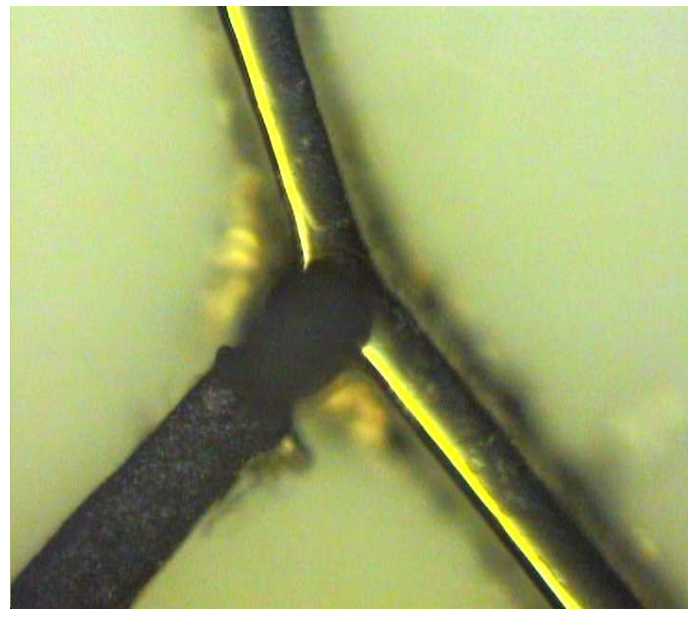

(b) Sem problemas

Figura C.4: Microposicionador $x y$ Bimetálico - Junções níquel-cobre.

Os protótipos obtidos não eram totalmente funcionais por causa de problemas de fabricação, como a falta de aderência entre as partes em níquel e cobre ou problemas durante a fotogravação. A fonte desses problemas está na dificuldade da remoção de fotoresiste, durante a revelação, de canais relativamente profundos e estreitos, o que impede uma eletrodeposição apropriada nas junções entre o níquel e o cobre, como pode ser visto na figura C.4.

Outro fotoresiste de mais fácil revelação, ainda em fase de caracterização no Laboratório de Microfabricação (LMF) do LNLS, está sendo testado e pode ser uma solução para a maioria dos problemas até agora enfrentados. 